Esquema numérico com reconstrução mínimos quadrados de alta ordem em malhas não-estruturadas para a formulação euleriana do transporte de partículas Olga Harumi Saito

TESE APRESENTADA AO INSTITUTO DE MATEMÁTICA E ESTATÍSTICA DA UNIVERSIDADE DE SÃO PAULO PARA OBTENÇÃO DO TÍTULO DE DOUTOR EM CIÊNCIAS

Área de Concentração: Matemática Aplicada Orientador: Prof. Dr. Luis Carlos de Castro Santos

São Paulo, janeiro de 2008. 



\title{
Esquema numérico com reconstrução mínimos quadrados de alta ordem em malhas não-estruturadas para a formulação euleriana do transporte de partículas
}

\author{
Este exemplar corresponde à redação \\ final da tese devidamente corrigida \\ e defendida por Olga Harumi Saito \\ e aprovada pela comissão julgadora.
}

São Paulo, 30 de janeiro de 2008.

Banca Examinadora:

- Prof. Dr. Luis Carlos de Castro Santos (Presidente) IME - USP

- Prof. Dr. Alexandre Megiorin Roma

IME - USP

- Prof. Dr. Marcos de Mattos Pimenta

EP - USP

- Prof. Dr. Fabio Saltara

EP - USP

- Prof. Dr. Admilson Teixeira Franco PPGEM - UTFPR 

Dedico esta tese aos meus pais Miyoiti e Miyoko, aos meus irmãos Edna, Sergio e Celso pelo amor e incentivo a mim sempre dados. 

"Sentir primeiro, pensar depois Perdoar primeiro, julgar depois Amar primeiro, educar depois Esquecer primeiro, aprender depois

Libertar primeiro, ensinar depois Alimentar primeiro, cantar depois Possuir primeiro, contemplar depois Agir primeiro, julgar depois Navegar primeiro, aportar depois Viver primeiro, morrer depois" Mario Quintana Mas agradecer sempre... 



\section{Agradecimentos}

À UTFPR - Universidade Tecnológica Federal do Paraná e aos colegas do DAMAT - Departamento Acadêmico de Matemática pela oportunidade, contribuindo para o meu crescimento profissional.

Ao meu orientador, Prof. Dr. Luis Carlos de Castro Santos, por sua orientação geral, empenho pessoal e encorajamento manifestado durante este trabalho, sempre de forma atenciosa e prestativa como foi também a sua orientação durante o meu mestrado.

À USP - Universidade de São Paulo que possibilitou, através do IME - Instituto de Matemática e Estatística, o meu crescimento científico, contribuindo para a minha carreira profissional.

Ao LCCA - Laboratório de Computação Científica Avançada do CCE - Centro de Computação Eletrônica da Universidade de São Paulo, que permitiu a realização das simulações de forma dinâmica. Ao Prof. Dr. Alexandre Megiorin Roma e ao Prof. Dr. Saulo Rabello Maciel de Barros, IME-USP, que nos cederam seus recursos computacionais.

Aos professores do IME-USP e EP-USP Escola Politécnica da Universidade de São Paulo pelo aprendizado. Aos funcionários das secretarias, biblioteca e setor de pósgraduação pela atenção e atendimento.

Ao Prof. Dr. Alexandre Megiorin Roma, IME-USP e ao Prof. Dr. Marcos de Mattos Pimenta, EP-USP, pela gentileza em aceitarem o convite para participarem do meu Exame de Qualificação e pelas valiosas sugestões que contribuíram para a elaboração desta tese.

Ao meu colega de doutoramento, Alessandro Alves Santana, pela parceria no desenvolvimento de parte do código. E a todos aqueles que de alguma forma, direta ou indiretamente, contribuiram para a conclusão desta tese o meu sincero agradecimento. 



\section{Resumo}

O estudo do transporte de partículas tem uma importância fundamental em diversas áreas de pesquisas como, por exemplo, na formação de gelo em uma aeronave pois pode afetar a sua sustentação e estabilidade. Tamanha é a preocupação com a segurança de vôo que diversos estudos têm sido realizados, resultando em códigos computacionais como o LEWICE nos Estados Unidos, TRAJICE no Reino Unido, ONERA na França e CANICE no Canadá. No Brasil, um dos estudo é feito pela EMBRAER em parceria com algumas instituições.

O objetivo deste trabalho é desenvolver um algoritmo que possa ser empregado na trajetória das partículas, utilizando uma formulação euleriana que elimina a dificuldade da semeadura de partículas específica da formulação lagrangiana na determinação da fração de volume da partícula $(\alpha)$. O método empregado é dos volumes finitos em malhas não-estruturadas cuja principal chave está na reconstrução mínimos quadrados de alta ordem com restrição nos contornos.

O desenvolvimento do trabalho engloba 3 etapas: definição da geometria e geração das malhas; utilização de um solver para o tratamento do escoamento do ar e obtenção do campo de velocidade; implementação e utilização do esquema numérico com reconstrução mínimos quadrados de alta ordem para simular o cálculo de $\alpha$ com imposição de condições limites apropriadas no contorno do corpo.

Os resultados dos testes realizados mostram que o esquema numérico com reconstrução mínimos quadrados pode ser empregado na resolução de equações que apresentam uma região de descontinuidade, como é o caso da região de sombra, reduzindo a largura da banda de difusão numérica e overshoots.

Palavras Chaves: reconstrução de alta ordem, malha não-estruturada, volumes finitos, advecção-difusão, formulação euleriana. 



\section{Abstract}

The particle transport study has a fundamental importance in diverse research area like in the icing accretion on an aircraft because that can affect its sustentation and stability. The concern is so big that many researches have been carried through, resulting in computational codes like the LEWICE in the United States, TRAJICE in the United Kingdom, ONERA in France and CANICE in Canada. In Brazil, one of the study has been made by the EMBRAER with some institutes.

The goal of this work is to develop an algorithm that can be used in the particles trajectory study, using an Eulerian method that eliminates the difficulty particle sowing, particular of the Lagrangian method, in the determination of the droplet fraction volume $(\alpha)$. This is made by the finite volume method on unstructured meshes whose main key is the high order reconstruction with restriction on the boundary.

The development of the work involves 3 stages: geometry definition and mesh generation; using code for the treatment of the air flow and obtained flow velocity; use of the high order numerical scheme least square reconstruction to simulate the $\alpha$ result with imposition of appropriate limit conditions in the body contour.

The realized simulations shown that Least Square method can be used in problem resolution that present descontinuos region like is shadow region reducing numerical diffusion and overshoots.

Key words: high order reconstruction, unstructured grid, finite volume method, advectiondiffusion, Eulerian formulation. 



\section{Índice}

Lista de Figuras $\quad$ xix

Lista de Tabelas $\quad$ xxvii

Lista de Símbolos $\quad$ xxix

1 Introdução 1

1.1 Motivação . . . . . . . . . . . . . . . . . . . 1

1.2 Estudos anteriores da formação de gelo . . . . . . . . . . . . . . . . 6

1.3 Descrição do processo de análise . . . . . . . . . . . . . . . . 9

2 Modelos matemáticos para o transporte de gotículas 13

2.1 Formulação lagrangiana . . . . . . . . . . . . . . . . . . 13

2.2 Formulação euleriana . . . . . . . . . . . . . . . . . 16

2.2.1 Condições de contorno e região de sombra . . . . . . . . . . . 18

2.2.2 Proposta do presente trabalho . . . . . . . . . . . . . . . . 20

3 Modelagem do escoamento 23

3.1 Método dos volumes finitos . . . . . . . . . . . . . . . 23

3.2 Definição da geometria e geração da malha computacional . . . . . . . . . 24

3.3 Solução numérica das equações de Navier-Stokes . . . . . . . . . . . . . . 27

3.3.1 Campo de velocidade na região retangular com cilindro em 2D . . . 31 
4 Reconstrução mínimos quadrados com alta ordem 43

4.1 As técnicas de reconstrução de alta ordem . . . . . . . . . . . . . . . . . . 43

4.2 O método da reconstrução mínimos quadrados . . . . . . . . . . . . . . . 45

4.3 Reconstrução da solução . . . . . . . . . . . . . . . . . . . . . . . . . . . . 45

4.3.1 Conservação da média . . . . . . . . . . . . . . . . . . . . . . 49

4.4 Condição de contorno forçada . . . . . . . . . . . . . . . . . . . 53

4.4.1 Contorno restrito para a condição de contorno de Dirichlet . . . . . 54

4.4.2 Restrição no contorno para a condição de contorno de Neumann . . 55

4.4.3 Restrição no contorno para condição de contorno de Robin . . . . . 56

4.5 Solução do problema mínimos quadrados restrito . . . . . . . . . . . . 57

4.6 Integração e cálculo do fluxo . . . . . . . . . . . . . . . . . . . 58

4.7 Evolução temporal . . . . . . . . . . . . . . . . . . . . . . 62

5 Verificação do esquema numérico $\quad 65$

5.1 Validação do cálculo da integral do fluxo para um volume de controle do interior . . . . . . . . . . . . . . . . . 65

5.1.1 Equação de difusão - Laplaciano no quadrado unitário . . . . . . . 66

5.1 .2 Advecção-difusão no canal retangular . . . . . . . . . . . . . . . . 72

5.2 Equação de advecção - cone de revolução . . . . . . . . . . . . . . . . . 76

5.3 Função de Harten . . . . . . . . . . . . . . . . . . . . . . . . . . . . . . 88

6 Aplicação no transporte euleriano de gotículas $\quad 93$

6.1 Teste 1 - Condição de contorno de Neumann no cilindro . . . . . . . . . . 93

6.1.1 Teste 2 - Condição de contorno definida pelo vetor velocidade . . . 97

6.1.2 Teste 3 - Condição de contorno definida pela componente da normal $n_{x} \ldots \ldots \ldots \ldots \ldots 10 \ldots \ldots \ldots$

6.2 Transporte da propriedade $\phi$ - velocidade constante $\mathrm{V}=(1,0) \ldots \ldots$. . . 112

6.2.1 Estudo do efeito da difusão numérica . . . . . . . . . . . . . . . . . 124

7 Conclusão

A Esquema de separação do vetor fluxo 
$\begin{array}{ll}\text { B Quadratura Gaussiana no triângulo } & 147\end{array}$

$\begin{array}{ll}\text { C Recursos computacionais utilizados } & 151\end{array}$

$\begin{array}{ll}\text { Referências Bibliográficas } & 160\end{array}$ 


\section{Lista de Figuras}

1.1 Esquema da formação de gelo no bordo de ataque de uma asa [67]. . . . . . 2

1.2 Regiões e componentes de uma aeronave sujeitos à formação de gelo [61]. . 3

1.3 Coeficiente de sustentação experimental, $C_{L}$, em função do ângulo de ataque, $\alpha$, de um perfil NACA63A415 sem formação de gelo (limpo) e com vários tipos de formação de gelo [61]. . . . . . . . . . . . . . . . 4

1.4 Trajetórias das gotículas, definição da eficiência de coleta local $\beta$, e dos limites de impacto superior $S_{U}$ e inferior $S_{L}[61] \ldots \ldots \ldots$

1.5 Esquema da resolução do problema de formação de gelo. . . . . . . . . . . 10

2.1 Linhas de corrente e trajetória de cada gotícula na formulação lagrangiana

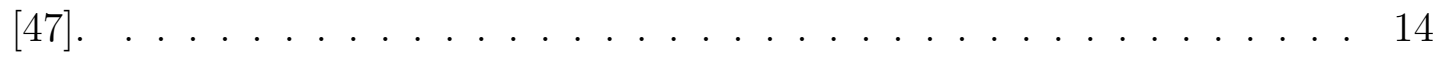

2.2 Balanço das forças atuando sobre a gotícula [63] . . . . . . . . . . . 14

2.3 Domínio computacional: geometria do aerofólio e contornos. . . . . . . . . 18

3.1 Volume de controle em uma malha não-estruturada: (a) centrado na célula; (b) centrado no vértice $[70] . \ldots \ldots \ldots$. . . . . . . . . . . . . 24

3.2 - - -Foco: construir esquemas numéricos para a solução das equações de transporte na formulação euleriana. . . . . . . . . . . . . . . . . . . . 25

3.3 Editor de malhas EMC2 $[56] . \ldots \ldots \ldots \ldots \ldots$

3.4 Volume de controle para a discretização espacial: Método dos Volumes Finitos Galerkin. . . . . . . . . . . . . . . . . . . 30

3.5 Domínio a ser estudado: cilindro de diâmetro $D=0.1016 \mathrm{~m}$ em uma região retangular $\Omega=[-2 D, 6 D] \times[-3 D, 3 D] \ldots \ldots \ldots \ldots \ldots$ 
3.6 Condição de contorno para o escoamento do ar: $2=$ simetria; $3=$ sólido; 4 $=$ saída e $5=$ entrada.

3.7 Nomenclatura dos contornos do domínio para a definição do número de partições. . . . . . . . . . . . . . . . . 34

3.8 zoom da malha m1. . . . . . . . . . . . . . . . . . . . 35

3.9 zoom da malha m2. . . . . . . . . . . . . . . . . . . . 36

3.10 zoom da malha m3. . . . . . . . . . . . . . . . . . . . 36

3.11 zoom da malha m4. . . . . . . . . . . . . . . . . . . . . 37

3.12 zoom da malha $m 5 \ldots \ldots \ldots$. . . . . . . . . . . . . . . . 37

3.13 zoom da malha $6 . \ldots \ldots \ldots \ldots$

3.14 Vetor velocidade na malha $m 3$ : (a) $R e=1.5 \times 10^{5}$ e (b) $R e=6.7 \times 10^{5}$. . 39

3.15 Magnitude da velocidade na malha $m 3$ : (a) $R e=1.5 \times 10^{5}$ e (b) $R e=6.7 \times 10^{5} .40$

3.16 Mach na malha $m 3$ : (a) $R e=1.5 \times 10^{5}$ e (b) $R e=6.7 \times 10^{5} \ldots \ldots$. . . 41

3.17 Campo de pressão na malha m3: (a) $R e=1.5 \times 10^{5}$ e (b) $R e=6.7 \times 10^{5} . .42$

4.1 Exemplo de possíveis estênceis no esquema ENO. . . . . . . . . . . . . . 44

4.2 (a) Volume de controle centrado na célula; (b) volume de controle centrado no vértice $[70] . \ldots \ldots \ldots$. . . . . . . . . . . . . . . . . . . . . 46

4.3 Estêncil de parte da malha para reconstrução de alta ordem: $2^{a}$, $3^{a}$ e $4^{a .}$ ordem, respectivamente: (a) do volume de controle centrado no vértice $i$ e (b) do volume de controle centrado na célula $i$, adaptado de [70] . . . . . . 47

4.4 Elementos triangulares com uma face em comum $\left(\overline{F_{1} F_{2}}\right) \ldots \ldots$. . . . . . . 47

4.5 Vizinhos da reconstrução de alta ordem com volume de controle centrado no vértice: $i_{c}$ é um vértice do contorno e $i$ é um vértice do interior, adaptado

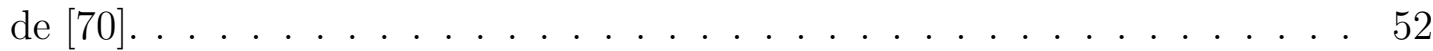

4.6 Localização dos pontos de Gauss para volume de controle adjacente ao contorno, adaptado de [70].

4.7 (a) Volume de controle centrado em 0; (b) $\overline{a b}$ e $\overline{b c}$ são as faces do VC para determinar o gradiente médio entre os vértices 0 e 1.

4.8 (a) Esquema para determinar os pontos de Gauss no esquema centrado no vértice; (b) número de ponto de Gauss relacionado à ordem de precisão da reconstrução. 
4.9 Procedimento no esquema de reconstrução mínimos quadrados com alta ordem.

5.1 Quadrado unitário $\Omega=[0,1] \times[0,1]$ : malha $\mathbf{A}$, 145 vértices, 286 triângulos e 392 faces.

5.2 Ordem de precisão da reconstrução da função $\phi(x, y)=\operatorname{sen}(\pi x) \operatorname{sen}(\pi y)$ para os volumes de controle do interior: norma $\mathrm{L}_{\infty} \ldots \ldots . . . . .$.

5.3 Ordem de precisão da reconstrução da função $\phi(x, y)=\operatorname{sen}(\pi x) \operatorname{sen}(\pi y)$ para os volumes de controle do interior: norma $\mathrm{L}_{1} \ldots \ldots$. . . . . . . . . . 68

5.4 Ordem de precisão da reconstrução da função $\phi(x, y)=\operatorname{sen}(\pi x) \operatorname{sen}(\pi y)$ para os volumes de controle do interior: norma $\mathrm{L}_{2} \ldots \ldots \ldots$. . . . . . .

5.5 Visualização em 2D da evolução da aproximação numérica de $\phi(x, y)=$ $\operatorname{sen}(\pi x) \operatorname{sen}(\pi y)$ no quadrado unitário, com recontrução de terceira ordem: (a) $\mathrm{t}=1 ;$ (b) $\mathrm{t}=10 ;$ (c) $\mathrm{t}=40 ;(\mathrm{d}) \mathrm{t}=80 ;$ (e) $\mathrm{t}=100 ;(\mathrm{f}) \mathrm{t}=200 ;(\mathrm{g}) \mathrm{t}$ $=400 ;(\mathrm{h}) \mathrm{t}=620 ;$ (i) média da solução exata $\phi(x, y)=\operatorname{sen}(\pi x) \operatorname{sen}(\pi y)$.

5.6 Visualização em 3D da evolução da aproximação numérica de $\phi(x, y)=$ $\operatorname{sen}(\pi x) \operatorname{sen}(\pi y)$ no quadrado unitário, com recontrução de terceira ordem: (a) $\mathrm{t}=1 ;$ (b) $\mathrm{t}=10 ;(\mathrm{c}) \mathrm{t}=40 ;(\mathrm{d}) \mathrm{t}=80 ;(\mathrm{e}) \mathrm{t}=100 ;(\mathrm{f}) \mathrm{t}=200 ;(\mathrm{g}) \mathrm{t}$ $=400 ;(\mathrm{h}) \mathrm{t}=620 ;(\mathrm{i})$ média da solução exata $\phi(x, y)=\operatorname{sen}(\pi x) \operatorname{sen}(\pi y)$.

5.7 Advecção-difusão em um canal retangular: (a) Função/condição inicial; (b) campo de velocidade $(1,0) \ldots \ldots \ldots \ldots$. . . . . . . . . . . .

5.8 Ordem de precisão da reconstrução da integral do fluxo no canal retangular: norma $\mathrm{L}_{\infty} \ldots \ldots \ldots \ldots \ldots \ldots \ldots$

5.9 Ordem de precisão da reconstrução da integral do fluxo no canal retangular: norma $\mathrm{L}_{1} \ldots \ldots \ldots \ldots \ldots \ldots$

5.10 Ordem de precisão da reconstrução da integral do fluxo no canal retangular: norma $\mathrm{L}_{2} . \ldots \ldots \ldots \ldots \ldots$. . . . . . . . . . . . . 76

5.11 Condição inicial para o cone de revolução. . . . . . . . . . . . . . . . . 77

5.12 Revolução de um cone: campo de velocidade definido por $u=-2 \pi(y-1)$ e $v=2 \pi(x-1) \ldots \ldots \ldots \ldots \ldots \ldots \ldots$

5.13 Visualização em 2D da rotação do cone de revolução com precisão de $4^{a}$ ordem, para $\Delta t=0.001$ : (a) distribuição inicial; (b) $\mathrm{t}=100 ;$ (c) $\mathrm{t}=200$; (d) $\mathrm{t}=300 ;(\mathrm{e}) \mathrm{t}=500 ;(\mathrm{f}) \mathrm{t}=700 ;(\mathrm{g}) \mathrm{t}=800 ;(\mathrm{h}) \mathrm{t}=900 ;(\mathrm{i}) \mathrm{t}=1000$.

5.14 Visualização em 3D da rotação do cone de revolução com precisão de $4^{a}$. ordem, para $\Delta t=0.001$ : (a) distribuição inicial; (b) $\mathrm{t}=100 ;$ (c) $\mathrm{t}=200$; (d) $\mathrm{t}=300 ;(\mathrm{e}) \mathrm{t}=500 ;(\mathrm{f}) \mathrm{t}=700 ;(\mathrm{g}) \mathrm{t}=800 ;(\mathrm{h}) \mathrm{t}=900 ;(\mathrm{i}) \mathrm{t}=1000$. 
5.15 Visualização em 3D do cone de revolução, usando reconstrução da solução com precisão de $4^{a}$ ordem: (a) condição incial (média nos volumes de controle); (b) após uma rotação completa; (b) após 5 rotações completas. 80

5.16 Corte de perfil no cone de revolução para $x=0.5$, na comparação após uma rotação, reconstrução da solução de $2^{a}, 3^{a}$ e $4^{a}$ ordem. . . . . . . . . . 80

5.17 Corte de perfil no cone de revolução para $x=0.5$, na comparação após uma rotação e 5 rotações, reconstrução da solução de $2^{a}$ ordem: (a) malha 60x60 (4545 vértices); (b) malha 120x120 (17890 vértices); (c) malha 240x240 (71119 vértices) . . . . . . . . . . . . . . . . . . . .

5.18 Corte de perfil no cone de revolução para $x=0.5$, na comparação após uma rotação e 5 rotações, reconstrução da solução de $3^{a}$ ordem: (a) malha 60x60 (4545 vértices); (b) malha 120x120 (17890 vértices); (c) malha 240x240 (71119 vértices). . . . . . . . . . . . . . . . . . .

5.19 Corte de perfil no cone de revolução para $x=0.5$, na comparação após uma rotação e 5 rotações, reconstrução da solução de $4^{a}$. ordem: (a) malha 60x60 (4545 vértices); (b) malha 120x120 (17890 vértices); (c) malha 240x240 (71119 vértices). . . . . . . . . . . . . . . . . . . .

5.20 Reconstrução linear da função de Harten: (a) função de Harten exata; (b) malha H1 (535 vértices); (c) malha H2 (3173 vértices); (d) malha H3 (27951 vértices). . . . . . . . . . . . . . . . . . . . 90

5.21 Corte horizontal em $y=0.0$ na reconstrução linear da Função de Harten: (a) malha H1 (535 vértices); (b) malha H2 (3173 vértices); (c) malha H3 (27951 vértices). . . . . . . . . . . . . . . . . .

5.22 Corte vertical em $x=0.0$ na reconstrução linear da função de Harten: (a) malha H1 (535 vértices); (b) malha H2 (3173 vértices); (c) malha H3 (27951 vértices). . . . . . . . . . . . . . . . . .

6.1 Transporte da propriedade $\phi$ : malha $m 5$ - 26866 vértices, campo de velocidade do NSC2KE para $R e=1.5 \times 10^{5}$ e reconstrução quadrática. . . . . . . 94

6.2 Vetores do campo de velocidade na região de sombra na Figura 6.1. . . . . 95

6.3 Valores da propriedade $\phi$ no cilindro: malha $m 5$ - 26866 vértices, campo de velocidade do NSC2KE para $R e=1.5 \times 10^{5}$ e reconstrução quadrática. .

6.4 Transporte da propriedade $\phi$ : malha $m 5$ - 26866 vértices e campo de velocidade do NSC2KE para $R e=6.7 \times 10^{5}$ e reconstrução quadrática. . . . . . 96

6.5 Vetores do campo de velocidade na região de sombra na Figura 6.4. . 96 
6.6 Valores da propriedade $\phi$ no cilindro: malha $m 5$ - 26866 vértices e campo de velocidade do NSC2KE para $R e=6.7 \times 10^{5}$ e reconstrução quadrática. . 97

6.7 Ponto de descolamento definido pelo vetor direção da velocidade. . . . . . . 98

6.8 Transporte do escalar $\phi$ : malha m3 - 55239 vértices, campo de velocidade do NSC2KE para $R e=1.5 \times 10^{5}$ e reconstrução linear. . . . . . . . . . . . . 98

6.9 Zoom da região de sombra - região atrás do cilindro da Figura 6.8 . . . . . . 99

6.10 Vetores do campo de velocidade na região de sombra na Figura 6.8. . . . . 99

6.11 Valores da propriedade $\phi$ no cilindro: malha m3 - 55239 vértices, campo de velocidade do NSC2KE para $R e=1.5 \times 10^{5}$ e reconstrução linear. . . . . 100

6.12 Definição das condições de contorno no cilindro através da componente da

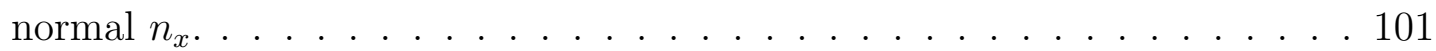

6.13 Transporte do escalar $\phi$ : malha $m 1$ - 3559 vértices, reconstrução linear. . . 102

6.14 Valores da propriedade $\phi$ no cilindro: malha $m 1$ - 3559 vértices, reconstrução linear. . . . . . . . . . . . . . . . . . . . . . . . 102

6.15 Transporte da propriedade $\phi$ : malha $m 1$ - 3559 vértices, reconstrução quadrática. . . . . . . . . . . . . . . . . . . 103

6.16 Valores da propriedade $\phi$ no cilindro: malha $m 1$ - 3559 vértices, reconstrução quadrática. . . . . . . . . . . . . . . . . . . . 103

6.17 Transporte do escalar $\phi$ : malha $m_{4}$ - 6724 vértices, reconstrução linear . 104

6.18 Valores da propriedade $\phi$ no cilindro: malha $m_{4}-6724$ vértices, reconstrução linear. . . . . . . . . . . . . . . . . . . . . . . . . . . 104

6.19 Transporte da propriedade $\phi$ : malha $m_{4}-6724$ vértices, reconstrução quadrática. . . . . . . . . . . . . . . . . . . 105

6.20 Valores da propriedade $\phi$ no cilindro: malha $m_{4}-6724$ vértices, reconstrução quadrática. . . . . . . . . . . . . . . . . . . 105

6.21 Transporte do escalar $\phi$ : (a) malha m2 - 13664 vértices, reconstrução linear; (b) malha $m 5$ - 26866 vértices, reconstrução linear. . . . . . . . . . . . . . 106

6.22 Valores da propriedade $\phi$ no cilindro: (a) malha m2 - 13664 vértices, reconstrução linear; (b) malha m5 - 26866 vértices, reconstrução linear. . . . 107

6.23 Transporte do escalar $\phi$ : malha m3 - 55239 vértices, reconstrução linear. 108

6.24 Valores da propriedade $\phi$ no cilindro: malha $m 3$ - 55239 vértices, reconstrução linear. . . . . . . . . . . . . . . . . . . . . . . . . . . . . . . 108

6.25 Perfis $p_{1}, p_{2}$ e $p_{3}$ no transporte da propriedade $\phi$, com condição de contorno definido pela componente da normal $n_{x} \ldots \ldots$. . . . . . . . . 109 
6.26 Perfis $p_{1}, p_{2}$ e $p_{3}$ no transporte da propriedade $\phi$ - campo de velocidade do NSC2KE: (a) malha m2 (13664 vértices), reconstrução linear; (b) malha m5 (26866 vértices), reconstrução quadrática. . . . . . . . . . . . . . . . . 110

6.27 Perfis $p_{1}, p_{2}$ e $p_{3}$ no transporte da propriedade $\phi$ - campo de velocidade do NSC2KE: malha m3 (55239 vértices), reconstrução linear. . . . . . . . . . . 111

6.28 Perfil $p_{3}$ no transporte da propriedade $\phi$ nas malhas $m 1$, m2 e m3, reconstrução quadrática: influência da malha na difusão numérica. . . . . . . . . 111

6.29 Transporte da propriedade $\phi$ na malha $m 1,3559$ vértices: reconstrução linear.114

6.30 Transporte da propriedade $\phi$ na malha $m 1,3559$ vértices: reconstrução quadrática. . . . . . . . . . . . . . . . . . . . . 114

6.31 Transporte da propriedade $\phi$ na malha $m 1,3559$ vértices: reconstrução cúbica . . . . . . . . . . . . . . . . . . . . . 115

6.32 Transporte da propriedade $\phi$ na malha m2, 13664 vértices: reconstrução linear. . . . . . . . . . . . . . . . . . . . . . 115

6.33 Transporte da propriedade $\phi$ na malha m2, 13664 vértices: reconstrução quadrática. . . . . . . . . . . . . . . . . 116

6.34 Transporte da propriedade $\phi$ na malha m2, 13664 vértices: reconstrução cúbica. . . . . . . . . . . . . . . . . . 116

6.35 Transporte da propriedade $\phi$ na malha m3, 55239 vértices: reconstrução linear. . . . . . . . . . . . . . . . . . . . 117

6.36 Transporte da propriedade $\phi$ na malha m3, 55239 vértices: reconstrução quadrática. . . . . . . . . . . . . . . . . 117

6.37 Transporte da propriedade $\phi$ na malha $m 3,55239$ vértices: reconstrução cúbica. . . . . . . . . . . . . . . . . . . . . 118

6.38 Transporte da propriedade $\phi$ na malha $m 4,6724$ vértices: reconstrução linear.118

6.39 Transporte da propriedade $\phi$ na malha m4, 6724 vértices: reconstrução quadrática. . . . . . . . . . . . . . . . . . . . . . 119

6.40 Transporte da propriedade $\phi$ na malha $m_{4}, 6724$ vértices: reconstrução cúbica. . . . . . . . . . . . . . . . . . . . . 119

6.41 Transporte da propriedade $\phi$ na malha $m 5,26866$ vértices: reconstrução linear. . . . . . . . . . . . . . . . . . . . 120

6.42 Transporte da propriedade $\phi$ na malha m5, 26866 vértices: reconstrução quadrática. 
6.43 Transporte da propriedade $\phi$ na malha $m 5,26866$ vértices: reconstrução

6.44 Transporte da propriedade $\phi$ na malha $m 6,76013$ vértices: reconstrução

6.45 Transporte da propriedade $\phi$ na malha m6, 76013 vértices: reconstrução quadrática.

6.46 Transporte da propriedade $\phi$ na malha m6, 76013 vértices: reconstrução cúbica. . . . . . . . . . . . . . . . . . . . 122

6.47 Valores da propriedade $\phi$ no cilindro: malha m2 - 13664 vértices, reconstrução linear.

6.48 Valores da propriedade $\phi$ no cilindro: malha m2 - 13664 vértices, reconstrução cúbica. . . . . . . . . . . . . . . . . . . . . . . . .

6.49 Perfis a serem analisados nas malhas $m 1-m 6$ : $p_{1}$ em $\mathrm{x}=0.0, p_{2}$ em x=D/2 (centro do cilindro), $p_{3}$ em $\mathrm{x}=\mathrm{D}$ (extremidade do diâmetro do cilindro) e $p_{4}$ em x=0.2.

6.50 Análise da difusão numérica na malha $m 1$, perfis $p_{1}, p_{2}, p_{3}$ e $p_{4}$, para: (a) reconstrução linear; (b) reconstrução quadrática; (c) reconstrução cúbica. . 125

6.51 Análise da difusão numérica na malha m2, perfis $p_{1}, p_{2}, p_{3}$ e $p_{4}$, para: (a) reconstrução linear; (b) reconstrução quadrática; (c) reconstrução cúbica. . 126

6.52 Análise da difusão numérica na malha $m 3$, perfis $p_{1}, p_{2}, p_{3}$ e $p_{4}$, para: (a) reconstrução linear; (b) reconstrução quadrática; (c) reconstrução cúbica. . 127

6.53 Análise da difusão numérica na malha $m_{4}$, perfis $p_{1}, p_{2}, p_{3}$ e $p_{4}$, para: (a) reconstrução linear; (b) reconstrução quadrática; (c) reconstrução cúbica. . 128

6.54 Análise da difusão numérica na malha $m 5$, perfis $p_{1}, p_{2}, p_{3}$ e $p_{4}$, para: (a) reconstrução linear; (b) reconstrução quadrática; (c) reconstrução cúbica. . 129

6.55 Análise da difusão numérica na malha $m 6$, perfis $p_{1}, p_{2}, p_{3}$ e $p_{4}$, para: (a) reconstrução linear; (b) reconstrução quadrática; (c) reconstrução cúbica. . 130

6.56 Análise da difusão numérica, perfil $p_{4}$, para as malhas: (a) $m 1$; (b) m2; (c) malha m3. . . . . . . . . . . . . . . . . . . . . . . . . . . 132

6.57 Análise da difusão numérica, perfil $p_{4}$, para as malhas m1, m2 e m3: (a) reconstrução linear; (b) reconstrução quadrática; (c) reconstrução cúbica. . 133

7.1 Estudo da difusão numérica: (a) traçado do perfil; (b) perfil "ideal"; (c) perfil obtido. . . . . . . . . . . . . . . . . . . . 136 
A.1 Elemento triangular $a b c$ e seus elementos adjacentes [33]. . . . . . . . . . 138

A.2 Ilustração dos sinais de propagação [33] . . . . . . . . . . . . . . . . . . . . 139

A.3 Esquematização para as condições de contorno [33] . . . . . . . . . . . . . 143

B.1 (a) Triângulo natural e suas coordenadas; (b) triângulo "associado" [16]. 147 


\section{Lista de Tabelas}

2.1 Formulações para o cálculo do coeficiente de coleta. . . . . . . . . . . . . . 21

3.1 Formato do arquivo amdba obtido através do EMC2. . . . . . . . . . . 27

3.2 Número de partições em cada segmento/cilindro do domínio apresentado na Figura 3.7. . . . . . . . . . . . . . . . . . . . 34

3.3 Número de elementos em cada malha, com número de partições dado pela Tabela 3.2 e respectivo tempo de execução para $R e=1.0 \times 10^{6}$. . . . . . 35

4.1 Número de pontos de Gauss por ordem de precisão da reconstrução de $\phi . \quad$. 54

4.2 Pontos de Gauss, normais e pesos usados no cálculo da integral do fluxo, $L_{1}=\left|x_{a}-x_{b}\right|$ e $L_{2}=\left|x_{b}-x_{c}\right| \ldots \ldots \ldots \ldots$. . . . . . . . . 61

5.1 Malhas utilizadas para a validação da precisão da integral do fluxo do Laplaciano em $\Omega=[0,1] \times[0,1]$. A malha $\mathbf{A}$ pode ser vista na Figura 5.1.

5.2 Ordem de precisão para a integral do fluxo nos VC's internos na equação de Poisson com solução analítica $\phi(x, y)=\operatorname{sen}(\pi x) \operatorname{sen}(\pi y)$. . . . . . . . . 67

5.3 Malhas utilizadas para a validação da precisão da integral do fluxo no canal retangular: $m$ é o número de partições no comprimento $L$ e $n$ é o número de partições na altura $h . \ldots \ldots$. . . . . . . . . . . . . . 73

5.4 Ordem de precisão da reconstrução usando as malhas da Tabela 5.3 para a integral do fluxo nos VC's internos na equação de advecção-difusão no canal retangular. . . . . . . . . . . . . . . . . . . . . . . 74

5.5 Resultados obtidos no teste realizado com o cone de revolução. . . . . . . . 81

5.6 Rotação do cone com reconstrução de $2^{a .}$ ordem. . . . . . . . . . . . . 82 
5.7 Rotação do cone com reconstrução de $3^{a}$ ordem. . . . . . . . . . . . . . . 82

5.8 Rotação do cone com reconstrução de $4^{a .}$ ordem. . . . . . . . . . . . . . . 83

5.9 Malhas utilizadas para o teste com a função de Harten: $n$ é o número de partições em cada lado do domínio $\Omega=[-1,1] \times[-1,1] \ldots \ldots$. . . . . . 89

6.1 Tempo de execução para a condição de contorno de Neumann no cilindro empregando a malha $\mathrm{m} 5 \ldots$. . . . . . . . . . . . . . . . . . . . 94

6.2 Testes com a componente da normal $n_{x}$ definindo a condição no contorno. 101

6.3 Transporte da propriedade $\phi$ na malha $m 1,3559$ vértices: tempo de execução e número de iterações. . . . . . . . . . . . . . . . . . . . . . . . . . . 112

6.4 Transporte da propriedade $\phi$ na malha m2, 13664 vértices: tempo de execução e número de iterações. . . . . . . . . . . . . . . . . . . . . . . . . 112

6.5 Transporte da propriedade $\phi$ na malha m3, 55239 vértices: tempo de execução e número de iterações. . . . . . . . . . . . . . . . . . . . . . . . . 113

6.6 Transporte da propriedade $\phi$ na malha $m 4,6724$ vértices: tempo de execução e número de iterações. . . . . . . . . . . . . . . . . . . . . . . . 113

6.7 Transporte da propriedade $\phi$ na malha $m 5,26866$ vértices: tempo de execução e número de iterações. . . . . . . . . . . . . . . . . . . . . . . . . 113

6.8 Transporte da propriedade $\phi$ na malha $m 6,76013$ vértices: tempo de execução e número de iterações. . . . . . . . . . . . . . . . . . . . . . . . 113

B.1 Quadratura Gaussiana no triângulo utilizando suas coordenadas e área [16]. 150 


\section{Lista de Símbolos}

\section{Abreviaturas}

$2 D$ : bidimensional

$3 D$ : tridimensional

CAD : Computer Aided Design

CIRA : Centro Italiano Ricerche Aerospaziali

DRA : Defence Research Agency

EMC2 : Edition de Maillages et the Contours en 2 Dimensions

EDO : Equação Diferencial Ordinária

EDP : Equação Diferencial Parcial

EMBRAER : Empresa Brasileira de Aeronáutica

ENO : Essentially Non-Oscillatory

LCCA : Laboratório de Computação Científica Avançada

LEWICE : LEWis ICE accretion program

LWC : Liquid Water Content

MVD : Median Volumetric Diameter

xxix 
MVF : Método dos Volumes Finitos

NASA : National Aeronautics and Space Administration

NSC2KE : 2D and Axi-Euler and Navier-Stokes Solver

ONERA : Office National d'Etudes et de Recherches Aérospatiales

INRIA : L'Institut National de Recherche en Informatique et en Automatique

IPT : Instituto de Pesquisas Tecnológicas

\section{Romano}

$A$ : área do volume de controle

CFL : número de Courant

$D$ ou $d$ : diâmetro da partícula

$L$ : comprimento característico

$c:$ corda

$n$ : normal outward-facing

$t:$ tempo

$u, v$ : componentes da velocidade

$V C$ : volume de controle

$x, y:$ coordenadas espaciais

$C_{D}$ : coeficiente de arrasto

Re : número de Reynolds

$K$ : parâmetro de inércia

Fr : número de Froude

$g$ : aceleração da gravidade 


\section{Grego}

$\alpha$ : ângulo de ataque; fração de volume da gotícula

$\beta$ : eficiência de coleta local

$\omega$ : velocidade angular

$\rho:$ massa específica

$\mu$ : viscosidade dinâmica

$\kappa$ : coeficiente de difusibilidade

$\nu$ : viscosidade cinemática

\section{Sobre-escrito}

$n$ : nível no tempo

$m$ e $n$ : expoentes nas médias e nos termos geométricos

\section{Subescrito}

$i$ : índice do vértice em toda a malha

$j$ : índice dos vértices vizinhos a um vértice

$d$ ou $p$ : referente à partícula/gotícula

$\infty$ : referente à condição de corrente livre (valores do ambiente)

$\mathrm{w}:$ water

a : air

avc : área do volume de controle

at : área total

adv : advecção 
dif : difusão

\section{Especiais}

$\frac{\partial}{\partial \vec{n}}$ : derivada parcial na direção do vetor normal

$\frac{\partial}{\partial x}$ : derivada parcial em relação a $\mathrm{x}$

$\frac{\partial}{\partial y}$ : derivada parcial em relação a y

$\frac{d}{d x}$ : derivada ordinária em relação a $\mathrm{x}$

$\frac{d}{d y}$ : derivada ordinária em relação a y

$\nabla$ : gradiente 


\section{Introdução}

\subsection{Motivação}

O estudo do transporte de partículas tem uma importância fundamental em diversas campos de pesquisa como na indústria petrolífera, área ambiental, química e aeronáutica.

$\mathrm{Na}$ indústria petrolífera, um dos estudos do transporte de partículas é na modelagem de derrames de petróleo. Entre os tipos de modelos de derrames de petróleo estão os modelos de trajetória que procuram predizer a posição da mancha de petróleo com intuito de combater a poluição e predição de riscos potenciais [50].

Na área ambiental, Freitas [18] estudou o transporte atmosférico de emissões em queimadas. Isso ocorre em conseqüência da queima de biomassa e gases, quando partículas são emitidas para a atmosfera e podem provocar mudanças no equilíbrio climático e biogeoquímico do planeta Terra em várias escalas.

Os sólidos particulados com propriedades controladas como pureza, distribuição por tamanho, forma cristalina, morfologia, área específica, porosidade, fluidez e compressibilidade tem incentivado o desenvolvimento de novos métodos e processos na preparação de partículas sólidas e, ainda, a inovação de processos clássicos pela Divisão de Química do IPT - Instituto de Pesquisas Tecnológica [84].

Saito [55] modelou o problema hidrodinâmico de um tanque de agitação mecânica e análise do comportamento de partículas sólidas envolvidas nesse processo. Uma formulação simplificada dos métodos tradicionais foi adotada e teve como objetivo descrever 
qualitativamente o processo hidrodinâmico através das equações de Navier-Stokes em coordenadas cilíndricas para uma representação simples do tanque agitado.

Para modelar a trajetória das partículas e analisar a influência do campo de velocidade foi usado um sistema de EDO's em uma formulação lagrangiana, o qual foi solucionado numericamente pelo Método das Diferenças Finitas. Uma análise da influência dos discos-atuadores, com base em sua localização, função representativa e comprimento na suspensão das partículas foi realizada.

Na indústria aeronáutica um dos interesses no estudo transporte de partículas (gotículas) está na formação de gelo em aeronaves, por ser um efeito responsável por vários acidentes aéreos. Por isso, tal fenômeno necessita de muita atenção por parte da engenharia aeronáutica e dos grupos que a estudam, bem como operadores e pilotos [11, 19, 20, 21, $62,74,75]$ a fim de garantir a segurança de vôo.

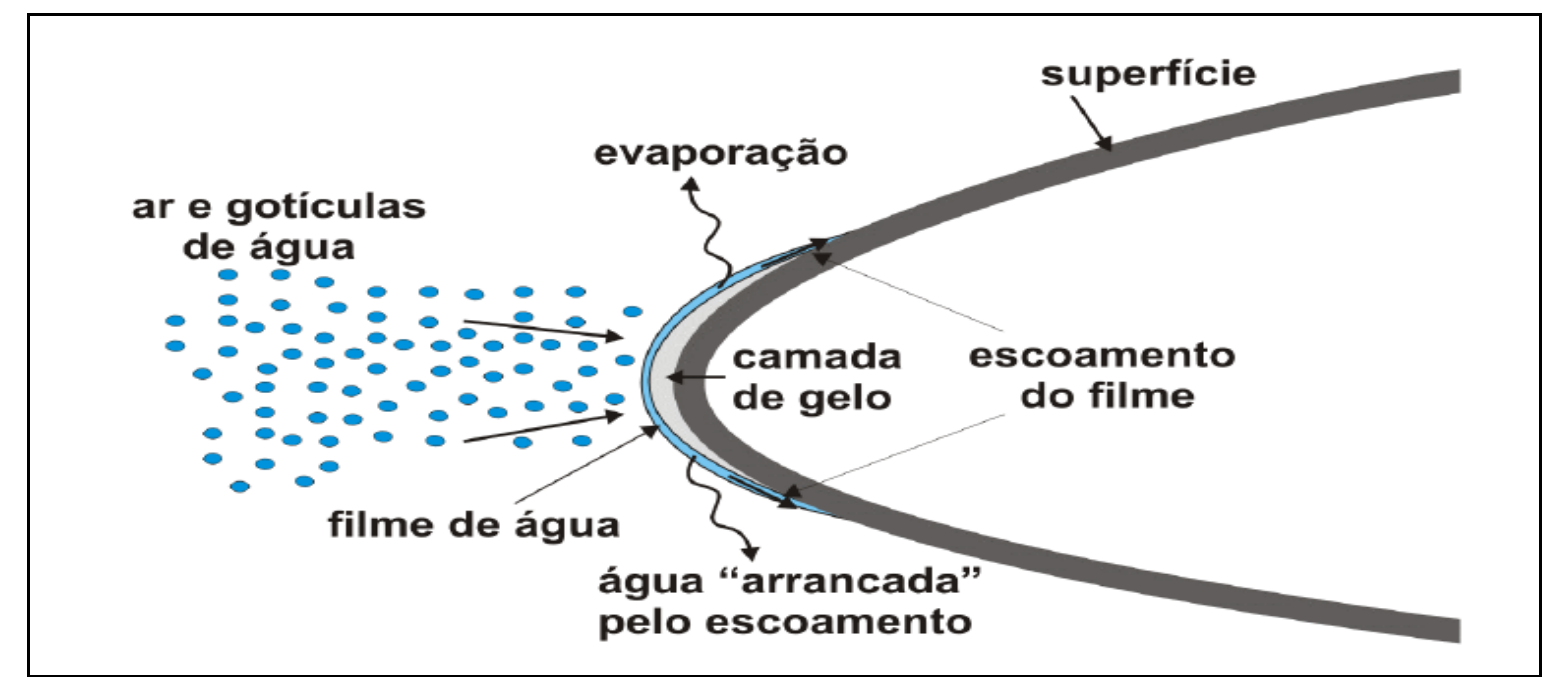

Figura 1.1: Esquema da formação de gelo no bordo de ataque de uma asa [67].

A formação de gelo pode ocorrer quando as aeronaves voam através de nuvens existentes na atmosfera e que contêm gotículas de água super geladas. Tais gotículas podem aderir à superfície da aeronave formando uma lâmina de água e, devido ao escoamento ocorre um processo termodinâmico nessa lâmina envolvendo convecção, evaporação e outros fenômenos térmicos. Com isto, uma parte dessa água se congela imediatamente com o impacto, a outra parte se desliza desprendendo-se ou voltando a se congelar. O processo se repete aumentando a espessura da camada de gelo, Figura 1.1. 
Diversos equipamentos e sistemas de uma aeronave podem ser afetados por tal fenômeno, como motores, antenas, hélices, asas, sondas, sensores e pára-brisas, que ficam expostos às condições externas, Figura 1.2, ocorrendo principalmente no bordo de ataque das asas e empenagens.

A formação de gelo nas asas diminui a sustentação e aumenta o arrasto e o peso total da estrutura. Conseqüentemente, é necessária maior tração do motor para manter o mesmo nível de vôo. Caso este acúmulo de gelo seja excessivo a redução de sustentação pode ser superior ao mínimo necessário para que a aeronave se mantenha equilibrada, levando a uma descida que pode ser catastrófica.

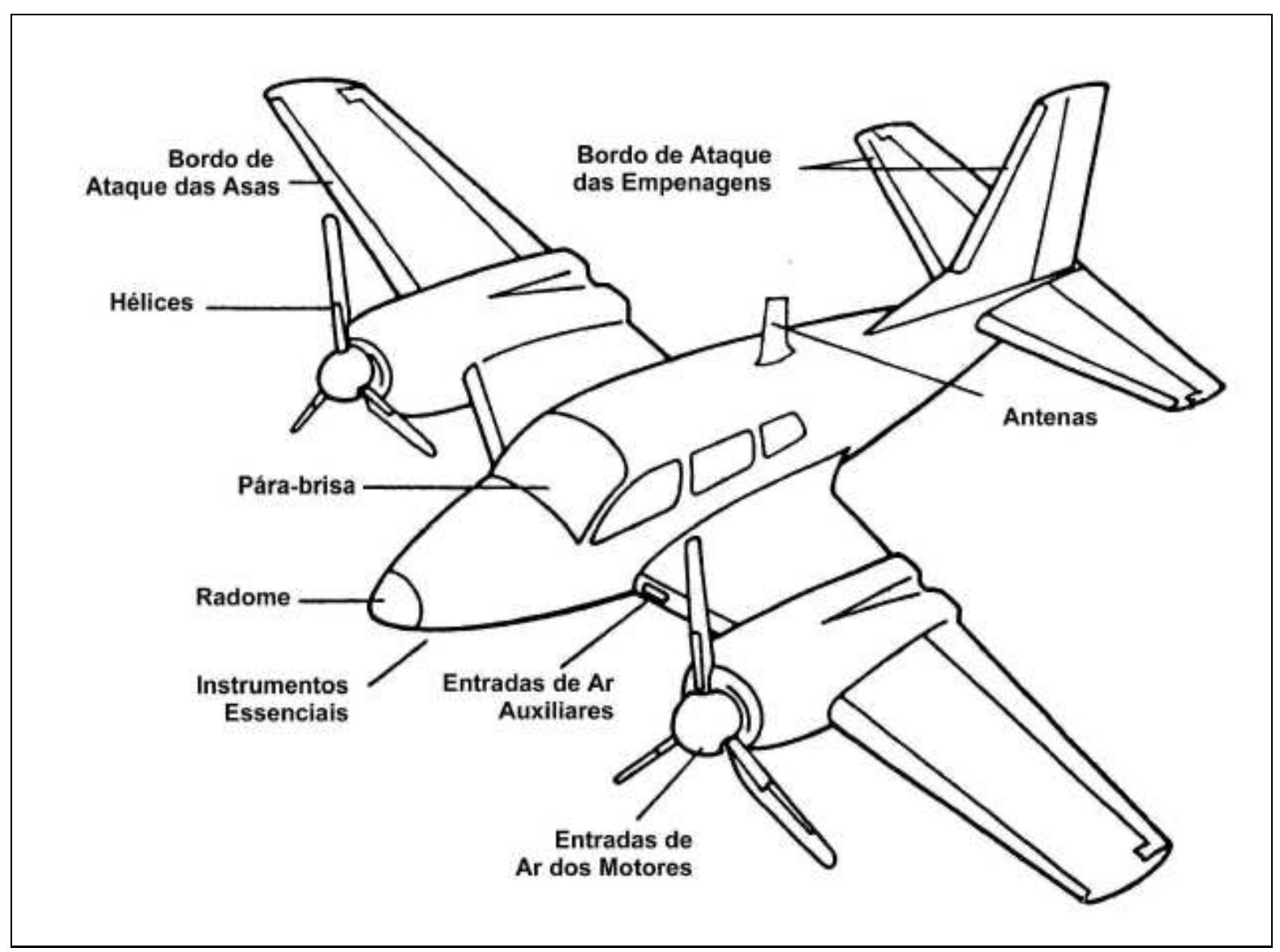

Figura 1.2: Regiões e componentes de uma aeronave sujeitos à formação de gelo [61].

Um exemplo experimental de degradação do desempenho aerodinâmico de um aerofólio causada por formação de gelo pode ser visto na Figura 1.3, que mostra o comportamento da queda do coeficiente de sustentação para as diversas formas de gelo. O gelo tipo glaze 
provoca uma queda mais acentuada do coeficiente de sustentação, $C_{L}$, comparando com as demais formas de gelo.

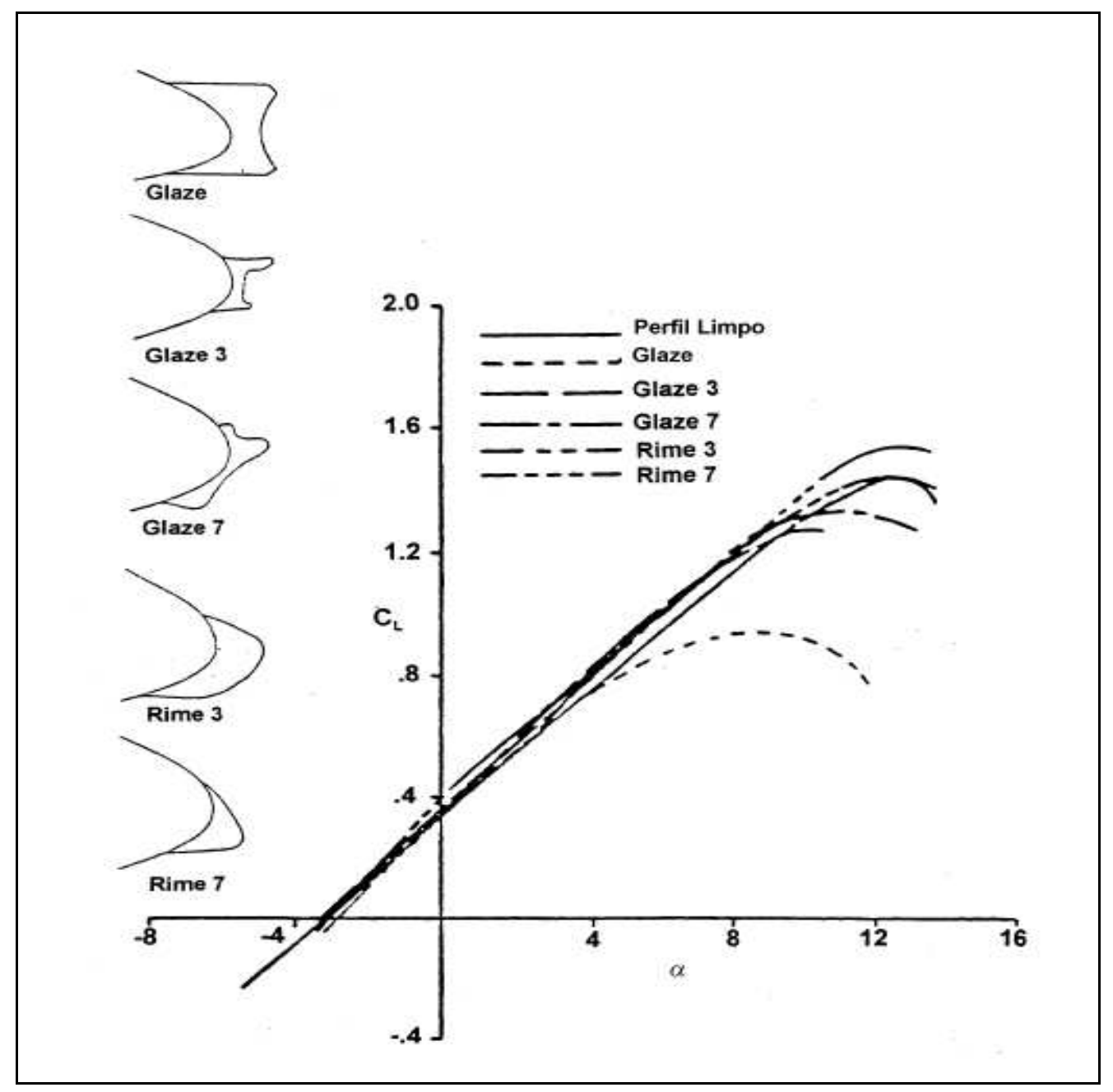

Figura 1.3: Coeficiente de sustentação experimental, $C_{L}$, em função do ângulo de ataque, $\alpha$, de um perfil NACA63A415 sem formação de gelo (limpo) e com vários tipos de formação de gelo [61].

O estudo do processo de formação de gelo em aeronaves envolve um conjunto de conceitos. Alguns deles serão apresentados a seguir para se ter um melhor entendimento do tratamento desse problema e que estarão presentes neste trabalho:

Conteúdo de água líquida ( $L W C$ - Liquid Water Content) numa nuvem, é definido como a concentração em massa de água líquida por volume de nuvem. O LWC, 
(1.1), determina a vazão máxima de água que pode impingir numa determinada área

$$
\dot{m}_{\infty}=L W C \cdot \vec{V}_{\infty} \cdot l \cdot d \vec{y}
$$

onde $\dot{m}_{\infty}$ é a vazão de massa de água que atravessa uma área $l \cdot d \vec{y}$ entre duas linhas de corrente normais à velocidade $V_{\infty}$.

Diâmetro volumétrico mediano ( $M V D$ - Median Volumetric Diameter) é um parâmetro importante no estudo da formação de gelo pois afeta diretamente a quantidade de água na superfície da aeronave, uma vez que gotículas maiores têm maior probabilidade de atingir a superfície do aerofólio, aumentando o que se denomina eficiência de coleta, ao contrário das gotículas menores, uma vez que as forças de arrasto daquelas são maiores que dessas. O MVD é a mediana da distribuição de diâmetros de gotículas de uma nuvem, ou seja, dada a distribuição dos diâmetros das gotículas, o MVD é o diâmetro para o qual a soma do volume das gotículas de água é menor do que $50 \%$ do volume total de água da amostra.

Eficiência de coleta local $(\beta$ ) representa, fisicamente, a fração do conteúdo de água líquida do ar (LWC) capturada por uma determinada posição da superfície da aeronave.

O estudo da eficiência de coleta das gotículas de água sobre um objeto iniciou-se com Langmuir e Blodgett, 1945, que caracterizou a eficácia da captação de um perfil geométrico dado através de um coeficiente de coleta.

A eficiência de coleta total é definida como a razão entre a massa real de água que atinge a superfície pelo total que ocorreria se as partículas seguissem trajetórias retilíneas, (1.2). A Figura 1.4 apresenta a trajetória das partículas e a definição de coleta local.

$$
\beta=\frac{d y}{d s}
$$

A região do aerofólio além dos limites superior e inferior de impacto das gotículas de água é denominada região de sombra.

Usando a formulação euleriana, a eficiência de coleta total é definida como sendo a razão entre a vazão de água coletada pela superfície inteira e a vazão de água em uma dada área no escoamento livre, (1.3). O movimento de um conjunto de gotículas é determinado como uma fração de massa de água $\alpha$ em cada ponto da malha computacional, fornecendo diretamente quais regiões do corpo têm impacto de água na superfície. 


$$
\beta=-\frac{\alpha(\vec{u} \cdot \vec{n})}{\vec{V}_{\infty}}
$$

onde $\vec{n}$ é o vetor normal à superfície e $\vec{V}_{\infty}$ é a velocidade na corrente livre.

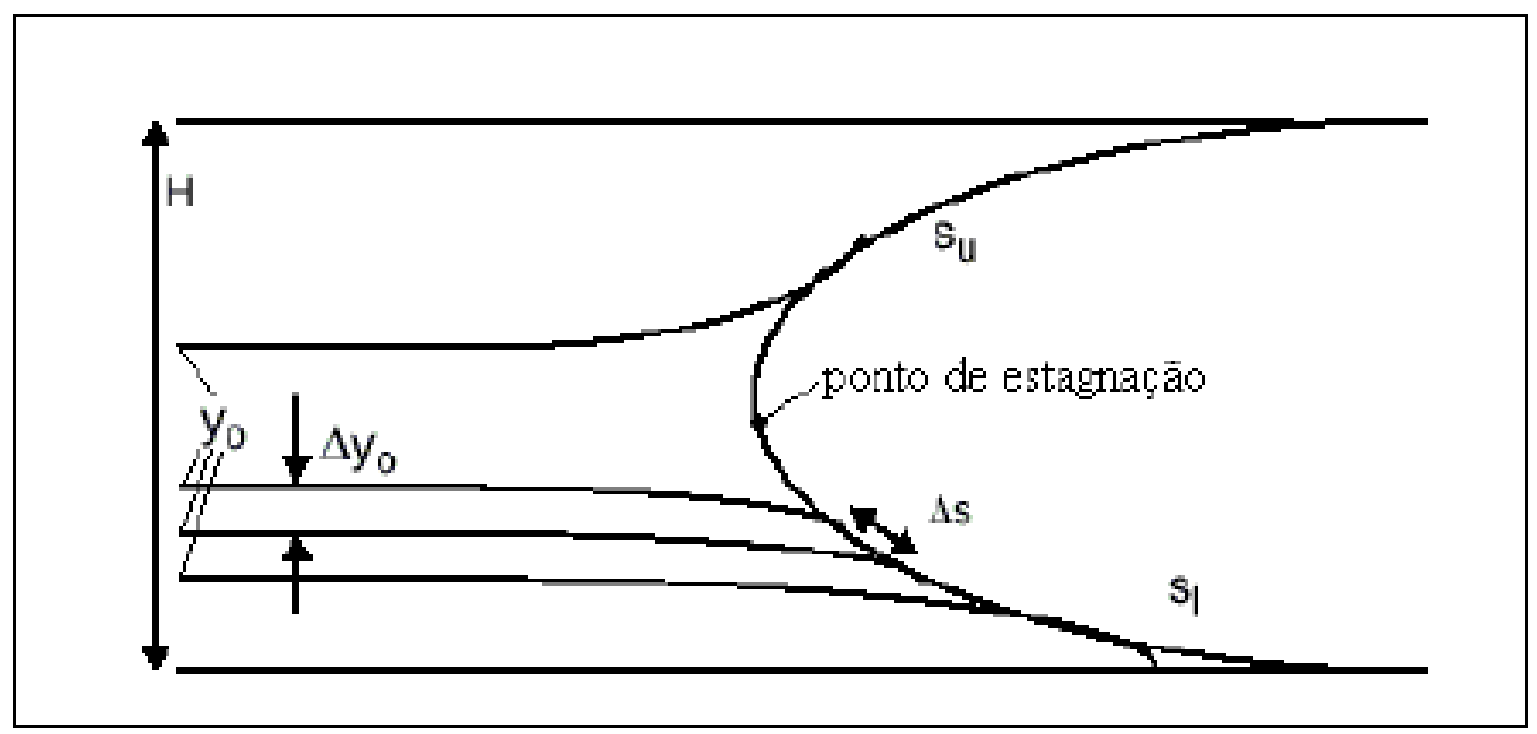

Figura 1.4: Trajetórias das gotículas, definição da eficiência de coleta local $\beta$, e dos limites de impacto superior $S_{U}$ e inferior $S_{L}[61]$.

Bourgault et al., 1997, desenvolveram e validaram um modelo euleriano tridimensional para cálculo de trajetórias que impingem na fuselagem dianteira de uma aeronave usando esta técnica.

\subsection{Estudos anteriores da formação de gelo}

A partir dos anos 1940, iniciou-se um estudo mais sistemático do fenômeno e de modelos para representar a formação de gelo em uma aeronave e os meios de prevenir ou solucionar este problema [75]. Nas décadas de 1940 e 1950, a NACA, agência dos Estados Unidos, testou em túnel de vento um aerofólio aquecido e estabeleceu um procedimento para avaliação da transferência de calor por convecção entre a superfície do aerofólio e o escoamento externo.

Taylor, 1940, desenvolveu uma formulação matemática para a trajetória da partícula e 
Langmuir \& Blodgett, 1947, desenvolveram significantes trabalhos experimentais. Testes de vôo foram realizados com o objetivo de construir sistemas de proteção contra a formação de gelo por Hardy, 1946, e Messinger, 1953.

Em 1950, a NACA calculou a distribuição do impacto das partículas no aerofólio, cilindro e esfera. Nos finais dos anos 1950, Gray et al. estudaram o processo no aerofólio NACA $65_{\mathrm{A}} 004$ e mostraram o efeito da formação de gelo e em 1958, Gray sugeriu uma equação empírica para predizer o coeficiente de arrasto na formação de gelo. Estas atividades de pesquisas constituiram uma ferramenta importante para simular a formação de gelo, limitadas pelos recursos computacionais da época.

Gray, 1961, publicou um estudo com o resumo da degradação do desempenho do aerofólio NACA devido à formação de gelo.

Em 1970 ocorreram grandes avanços nas pesquisas, principalmente no NASA Lewis Research Institute e em 1983 foi desenvolvido o modelo de formação de gelo 2D, LEWICE, no Instituto de Pesquisa da Universidade de Dayton.

Guffond, 1990, trabalhou na predição da formação de gelo 2D no ONERA, na França. Wright, Gent e Guffond, 1997, comparam os programas para previsão de formação de gelo das agências aeroespaciais governamentais da Inglaterra, França e EUA, TRAJICE2 [21, 78], ONERA2D [87], LEWICE [74], respectivamente. Chegaram a conclusão que eles têm o mesmo grau de desvio em relação às medições no estudo da formação de gelo.

Wright e Chung, 1999, estabeleceram um método para comparações de formas de gelo baseado nos efeitos de degradação da performance aerodinâmica, definindo critérios objetivos para análise do acúmulo de gelo.

De uma forma geral, a partir dos anos 1980, trabalhos contínuos de investigação da operação de aeronaves em condições naturais de formação de gelo têm sido desenvolvidos e vários métodos práticos usados para prevenir a formação de gelo (sistemas anti-gelo) ou remoção de gelo já formado (sistema de degelo). Estimuladas pelas falhas nos sistemas de proteção e a necessidade de vôo em áreas de formação de gelo, novas pesquisas são estimuladas incluindo a NASA (Estados Unidos) [34, 74], DRA (Reino Unido), ONERA (França) [87], Bombardier (Canadá) [80] e CIRA (Itália) [79], entre outros.

No Brasil, um dos principais estudos tem sido desenvolvidos pela EMBRAER em parceria com alguns institutos de pesquisas $[57,58,14]$. 
O estudo da formação de gelo, é uma parte importante do desenvolvimento do projeto de uma aeronave. Neste sentido, além dos estudos analíticos experimentais, tem-se destacado a simulação numérica, como visto anteriormente, que é feita de uma forma tradicional nos códigos NASA-LEWICE [74, 77] e ONERA [87] que trabalham com a formulação lagrangiana, conhecida também como Método de Localização Partícula por Partícula (Discrete Particle Method) [63, 64, 65, 66, 67].

Com o avanço de CFD - Computational Fluid Dynamics (DFC - Dinâmica dos Fluidos Computacional), o cálculo do impacto das partículas de água sobre um objeto, por exemplo em um aerofólio, pode ser proposta de uma forma alternativa usando uma formulação euleriana, conhecida também como Passive Scalar Transport Approach que tem como principal vantagem a possibilidade de calcular os limites de impacto (impingement limits) em configurações de aerofólios com multi-elementos como é o caso do FENSAP-ICE $[9,29,42,82,86]$ e da formulação euleriana do Fluent [76].

A abordagem usando a formulação euleriana ainda é pouco utilizada nos códigos de formação de gelo, mas vem ganhando bastante espaço devido à necessidade de se resolver problemas com geometrias mais complexas.

Além da vantagem já citada, a formulação euleriana elimina a dificuldade de semeadura específica da formulação lagrangiana, na obtenção da fração volumétrica usada para definir o coeficiente de coleta $(\beta)$, que permite estudar a forma do gelo em um corpo.

Na formulação lagrangiana para se obter uma boa resolução é necessário um número muito grande de partículas, lançadas de uma região que atinja a superfície de interesse sobre o aerofólio. Neste caso, tanto deve-se controlar o número de partícula quanto a região da qual elas são lançadas, enquanto que na formulação euleriana a malha utilizada para a solução do escoamento é a própria malha usada para a análise do transporte das partículas.

Como comentado, uma das etapas importantes da formação de gelo em uma aeronave é o estudo da eficiência de coleta local que permite determinar a região limite no aerofólio na formação de gelo e para a sua determinação é preciso resolver uma equação do tipo advectiva com termo forçante.

A maioria dos pacotes comerciais possuem aproximação de segunda ordem, que associado à necessidade de uma malha muito refinada para a solução adequada do problema, 
resulta em um alto custo computacional por iteração, é o caso do FENSAP-ICE quanto do FLUENT, necessitando uma adaptação para a região de sombra.

Os métodos de alta ordem têm-se apresentado como ferramentas alternativas a serem aplicadas nestes problemas com o intuito de reduzir o custo computacional. Dentre estes métodos, tem-se o método de reconstrução mínimos quadrados de alta ordem baseado na reconstrução mínimos quadrados em malhas não-estruturadas, implementado diretamente na mesma, sem a necessidade de busca de um estêncil conveniente.

O objetivo deste trabalho é implementar e avaliar o potencial do uso de método de alta ordem na resolução de problemas de advecção-difusão 2D que tem como motivação a resolução do transporte de partículas em formulação euleriana na determinação da fração de água líquida $\alpha$.

\subsection{Descrição do processo de análise}

A formação do gelo no bordo de ataque de um perfil aerodinâmico envolve basicamente seis etapas [61, 66, 67, 77], que são:

- definição da geometria do perfil;

- geração da malha sobre o domínio;

- obtenção do escoamento do ar sobre o perfil e cálculo de camada limite para os efeitos viscosos e de transferência de calor;

- cálculo das trajetórias das partículas de água que colidem com a superfície para a obtenção da fração de água líquida $(\alpha)$ - foco deste trabalho;

- cálculo do coeficiente de coleta $(\beta)$;

- balanços de energia e massa em cada volume de controle definido sobre a superfície para obter a quantidade de gelo formada e modificação da geometria em função do acúmulo de gelo.

Como estas partículas são gotas de água, a partir de agora elas serão denominadas gotículas. 
Uma estrutura do foco deste trabalho é apresentada na Figura 1.5 e consiste de, uma vez definida a geometria, gerar a malha sobre o domínio estabelecido e criar uma estrutura de dados. Através de um solver solucionar o escoamento obtendo as componentes da velocidade do ar. A equação que define a trajetória das gotículas será discretizada pelo método dos volumes finitos em malhas não-estruturadas utilizando reconstrução mínimos quadrados de alta ordem na reconstrução dos fluxos nas faces dos volumes de controle, com o devido tratamento do contorno. Os valores das componentes da velocidade são passados para a rotina que irá solucionar esta equação, com visualização dos resultados através do aplicativo Tecplot.

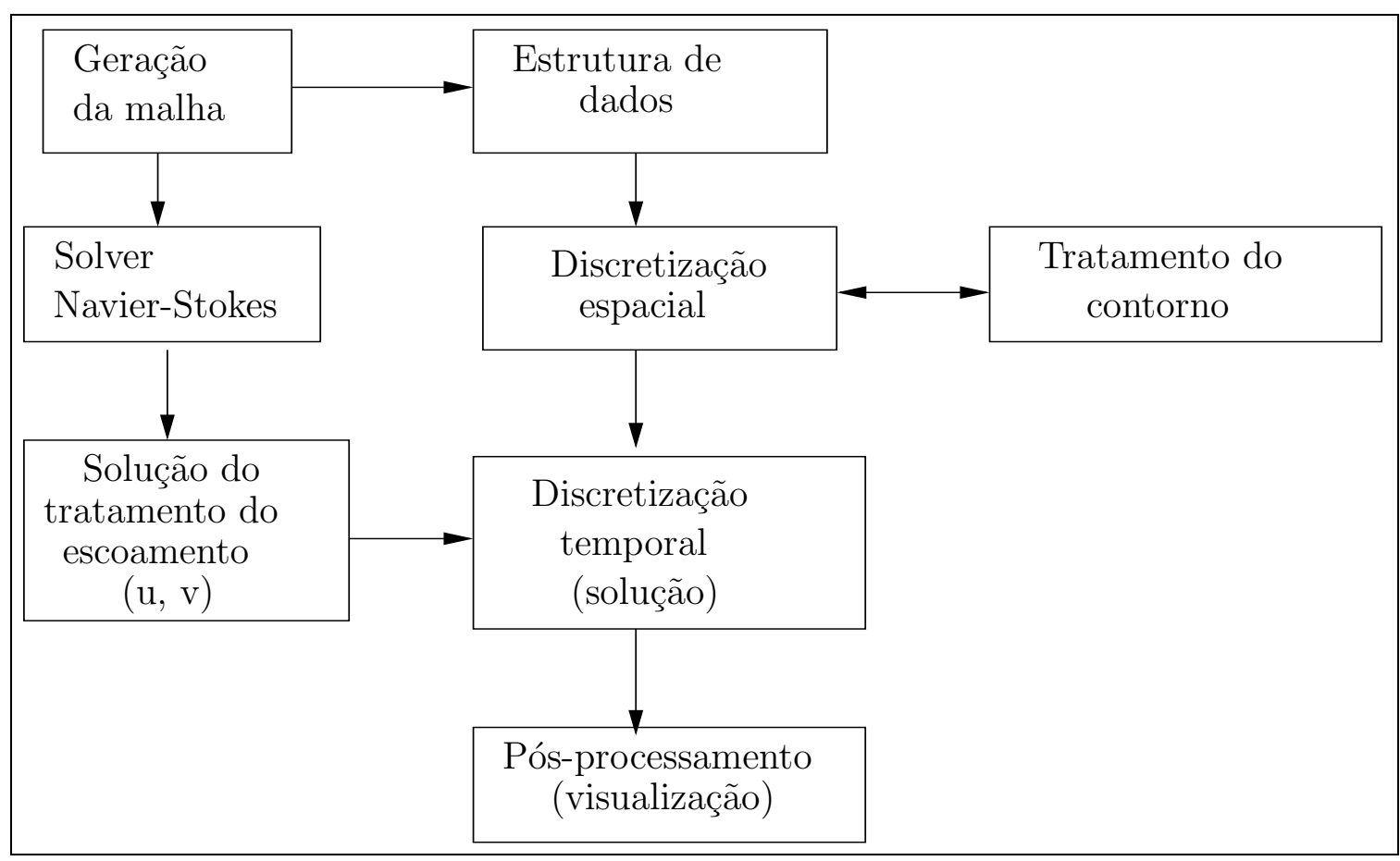

Figura 1.5: Esquema da resolução do problema de formação de gelo.

O presente trabalho está esquematizado da seguinte forma:

O Capítulo 2 apresenta as equações do transporte de gotículas para a obtenção da fração de água líquida $(\alpha)$ usada no cálculo da eficiência de coleta $(\beta)$.

O Capítulo 3 traz a modelagem do escoamento através do solver NSC2KE e o gerador de malha EMC2. O uso de malhas não-estruturadas e a técnica dos volumes finitos em uma formulação centrada no vértice, cell-vertex, são apresentados. 
O Capítulo 4 apresenta o esquema numérico com reconstrução mínimos quadrados de alta ordem, com a conservação da média, o cálculo dos termos geométricos e a restrição forçada no contorno do domínio para reconstrução de $2^{\text {a. }}, 3^{\text {a. }}$ e $4^{\text {a. }}$ ordem. O sistema linear obtido é resolvido pelo método de Eliminação de Gauss com pivoteamento parcial. Para fechar o capítulo é mostrada a técnica de integração do fluxo para a parte advectiva e parte difusiva da equação de advecção-difusão. O método de Runge-Kutta multi-estágios é empregado para a evolução temporal com coeficientes adequados ao problema físico.

O Capítulo 5 apresenta verificação do método dos volumes finitos com reconstrução de alta ordem. A metodologia para medir a precisão do método é descrita e os testes foram feitos para um domínio quadrado e são testados alguns casos usando funções $C^{\infty}$, descontínuas, equações de advecção, difusão e advecção-difusão para análise do comportamento dos resultados de acordo com a ordem de reconstrução.

O Capítulo 6 mostra os resultados da aplicação do método de reconstrução de alta ordem na análise da difusão numérica na simulação do transporte de um escalar passivo $\phi$ em uma região retangular com um cilindro em seu interior. Para estudar o efeito da difusão numérica em regiões do domínio uma análise dos perfis é feita. Este estudo permitirá em um trabalho futuro o emprego do método de reconstrução de alta ordem para o fim proposto.

O Capítulo 7 apresenta um resumo do trabalho e as presentes conclusões sobre o esquema numérico desenvolvido. 
CAPÍtulo 2

\section{Modelos matemáticos para o transporte de gotículas}

Este capítulo traz a formulação lagrangiana que analisa a trajetória gotícula a gotícula e apresenta a formulação euleriana, objeto deste estudo, que trata as gotículas como fase dispersa. O destaque é para o tratamento das condições de contorno.

\subsection{Formulação lagrangiana}

Os limites da região de impacto das gotículas sobre o aerofólio e a eficiência de coleta na formulação lagrangiana são obtidos através da análise da trajetória de cada gotícula que adere à superfície do corpo [64, 65, 66, 67, 74].

Para isto, algumas hipóteses são feitas. Dentre elas, cada gotícula é considerada como uma massa pontual, que não influencia o escoamento mas que sofre o arrasto aerodinâmico, e que não altera o campo de velocidade. A Figura 2.1 mostra a trajetória e as linhas de corrente do escoamento.

Com base nas hipóteses e considerando-se arrasto (D) e a aceleração da gravidade (g), um balanço de forças pode ser feito fornecendo as equações do movimento para as trajetórias das gotículas de massa $m$, Figura 2.2 .

$$
m \ddot{x}=-D \cos \gamma+m g \operatorname{sen} \theta
$$




$$
m \ddot{y}=-D \cos \gamma+m g \cos \theta
$$

onde $\theta$ é o ângulo de ataque e

$$
\gamma=\tan ^{-1}\left[\left(\dot{y}_{d}-v_{a}\right) /\left(\dot{x}_{d}-u_{a}\right)\right]
$$

sendo $\left(\dot{x}_{d}, \dot{y}_{d}\right)$ as componentes da velocidade da gotícula e (u, v) as componentes da velocidade do escoamento na posição da gotícula.

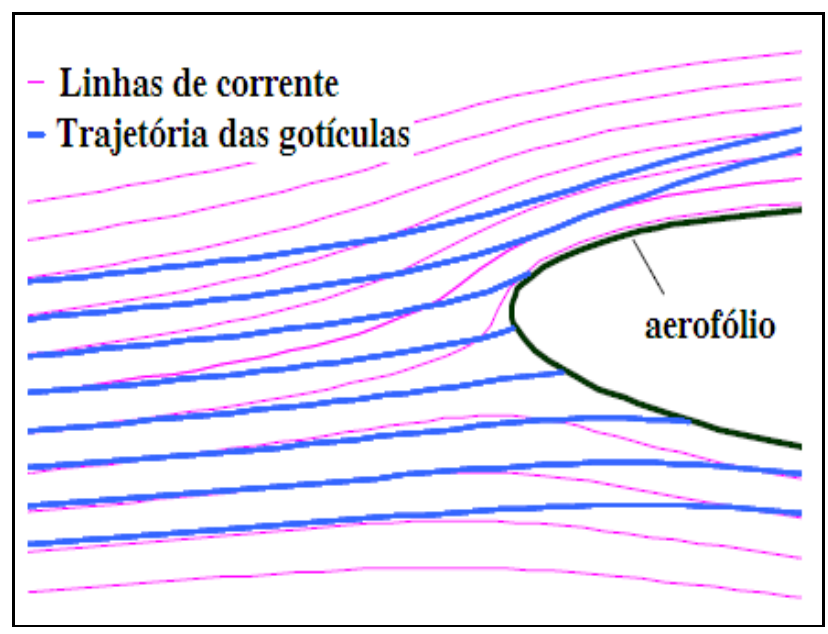

Figura 2.1: Linhas de corrente e trajetória de cada gotícula na formulação lagrangiana $[47]$.

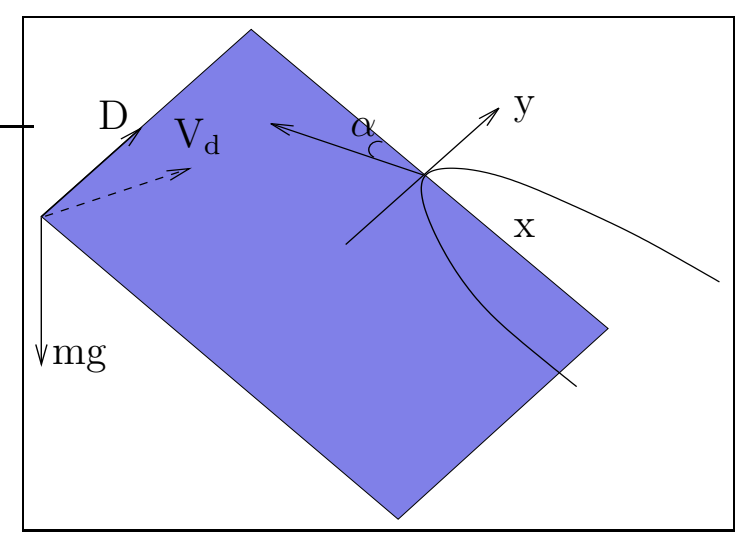

Figura 2.2: Balanço das forças atuando sobre a gotícula [63].

O arrasto é definido como 


$$
D_{d_{d}}=C_{D_{d}} \frac{\rho_{a} V^{2}}{2} A_{d}
$$

onde,

$d_{d}$ é o diâmetro da gotícula;

$A_{d}$ é a área projetada da gotícula;

$V=\sqrt{\left(\dot{x}_{d}-u_{a}\right)^{2}+\left(\dot{y}_{d}-v_{a}\right)^{2}}$.

Supondo-se que o formato das gotículas assemelha-se ao de uma esfera rígida, utiliza-se a correlação dada por [72]:

$$
C_{D_{d}}=\frac{24}{R e_{d}}+\frac{6}{1+\left(R e_{d}\right)^{0.5}}+0.4
$$

O número de Reynolds da gotícula, dado por $R e_{d}=V d_{d} / v$, varia entre 0 e $2 \times 10^{5}$, justificado por se tratar do movimento relativo entre o ar e a pequena esfera.

As equações do movimento podem ser reescritas

$$
\begin{aligned}
& \frac{\partial}{\partial t} \dot{x}=-\frac{D}{m} \cos \gamma+g \operatorname{sen} \theta \\
& \frac{\partial}{\partial t} \dot{y}=-\frac{D}{m} \cos \gamma+g \cos \theta
\end{aligned}
$$

A velocidade inicial para as gotículas considerada é a mesma velocidade do escoamento, suficientemente afastada do corpo, de tal forma que o fluxo nesta região não tenha sofrido nenhuma perturbação.

O sistema de equações obtido, devido à não-linearidade, é resolvido por um esquema numérico uma vez que o arrasto depende da velocidade do escoamento na posição da gotícula e essa por sua vez, depende da própria velocidade. Silveira [66] utiliza o método preditor-corretor Euler Modificado.

Os limites da região de impacto são calculados com um processo iterativo, iniciando por uma trajetória que atinge o corpo. Na seqüência, uma trajetória que passa acima do corpo para o limite superior e então a trajetória intermediária entre estas duas é calculada. Se essa atingir o corpo será a nova trajetória que atinge o corpo, caso contrário será a nova trajetória que passa por cima do corpo. O processo se repete até que a distância entre as posições iniciais da trajetória da gotícula que atinge a geometria e a que passa acima dela 
seja menor que um valor especificado. Analogamente, se repete o processo para o limite inferior.

\subsection{Formulação euleriana}

A formulação euleriana para a trajetória das gotículas foi introduzida por Bourgault et al. [9], em 1999 e está implementado no código FENSAP-ICE [42, 86]. É um modelo de dois fluidos constituído das equações de Euler ou Navier-Stokes para o ar, acrescentadas das equações para as gotículas: equação da continuidade e do momento.

O método é baseado nas mesmas equações da formulação lagrangiana mas expresso por um conjunto de 2 EDP's:

- para a concentração das gotículas de água, como a equação de continuidade;

- para a aceleração das gotículas, como a equação de momento.

Quando se modela a interação do escoamento do ar com as gotículas, um grande número de fenômenos e forças podem ser consideradas no estudo de situações de formação de gelo em uma aeronave em vôo, em [9] diz ser de bom senso supor que:

- as gotículas são esféricas e sólidas e elas não se deformam para o diâmetro volumétrico médio da amostra da distribuição de tamanho;

- não há quebra das gotículas com a aceleração, nem coalescência entre as gotículas por não ser significante em situações de formação de gelo;

- as gotículas não tem sustentação e nem momento aerodinâmico. As únicas forças que atuam sobre elas são devidas ao arrasto aerodinâmico, gravidade e empuxo;

- é aplicado o arrasto para uma esfera estacionária e não há efeitos transientes com a mudança do arrasto;

- a evaporação das gotículas é desprezada;

- o escoamento (compressível ou incompressível) é contínuo em torno das gotículas e não há interação entre o mesmo e as gotículas pois a concentração das gotículas de 
água são suficientemente pequenas, assim o escoamento aerodinâmico e das gotículas podem ser tratados como sistemas independentes;

- efeitos da turbulência sobre a gotícula podem ser negligenciados.

Com isto, a solução do escoamento e das gotículas podem ser resolvidos separadamente.

Em duas dimensões, para uma gotícula de diâmetro $d$, as equações de conservação são expressas por (2.7) e (2.8).

- Conservação da concentração de água

$$
\frac{\partial \alpha \rho}{\partial t}+\nabla \cdot\left(\alpha \rho u_{d}\right)=0
$$

semelhante à equação da continuidade.

- Conservação da aceleração das gotículas

$$
\frac{\partial \alpha \rho u_{d}}{\partial t}+\nabla \cdot\left(\alpha \rho u_{d} \otimes u_{d}\right)=K \alpha \rho\left(u_{a}-u_{d}\right)+\alpha \rho F
$$

semelhante à equação do momento, onde:

$\otimes$ é o produto diádico;

$\alpha(\mathrm{x}, \mathrm{t})$ é o valor médio da fração do volume da água;

$u_{d}$ é o valor médio da velocidade da gotícula;

$u_{a}$ é a velocidade do ar;

$\rho$ é a densidade da água. $K=\rho d^{2} U_{\infty} / 18 L \mu$ é o parâmetro de inércia;

$F$ é o termo forçante.

Reescrevendo (2.8), usando variáveis adimensionais , como na forma não-conservativa, tem-se

$$
\frac{\partial \alpha \rho u_{d}}{\partial t}+\nabla \cdot\left(\alpha \rho u_{d} \otimes u_{d}\right)=\underbrace{\frac{C_{D} R e_{d}}{24 K} \alpha \rho\left(u_{a}-u_{d}\right)}_{(I)}+\underbrace{\alpha \rho\left(1-\frac{\rho_{a}}{\rho_{d}}\right) \frac{1}{F r^{2}} g}_{(I I)}
$$

onde

(I) corresponde à força de arrasto do ar sobre as gotículas;

$(I I)$ corresponde à força de empuxo e da gravidade; 
Após a adimensionalização, tem-se:

$\alpha$ é a fração de água líquida adimensionalizada;

$\rho_{d}$ é a densidade da água;

$\rho_{a}$ é a densidade do ar;

$\left.R e_{d}=\rho d U_{\infty}\left|u_{a}-u_{d}\right|\right) / \mu$ é o número de Reynolds das gotículas;

$C_{D}=\left(24 / R e_{d}\right)\left(1+0.015 R e_{d}^{0.687}\right)$ para $R e \leq 1000$ e $C_{D}=0.4$ para $R e_{d}>1000$ é o coeficiente de arrasto para gotículas esféricas;

$F r=U_{\infty} / \sqrt{L g}$ é o número de Froude;

$U_{\infty}$ é a velocidade do ar para o infinito;

L é o comprimento característico (se for o caso de um aerofólio, é o comprimento da sua corda);

$\mu$, a viscosidade dinâmica do ar.

A velocidade adimensional do ar, $u_{a}$, pode ser obtida através da solução das equações de Navier-Stokes, por meio de um solver.

\subsubsection{Condições de contorno e região de sombra}

As condições de contorno apropriadas devem ser impostas no domínio para resolver $(2.7)$ e $(2.9)$.

Há duas classes de condições de contorno que podem ser vistas na Figura 2.3: contorno do domínio externo e o contorno do aerofólio. A primeira é relativamente simples, que será apresentada por $\Gamma_{1}$, contorno de entrada e $\Gamma_{2}$, contorno de saída. Mas, o mesmo não ocorre com a segunda, $\Gamma_{3}$, contorno sobre o aerofólio.

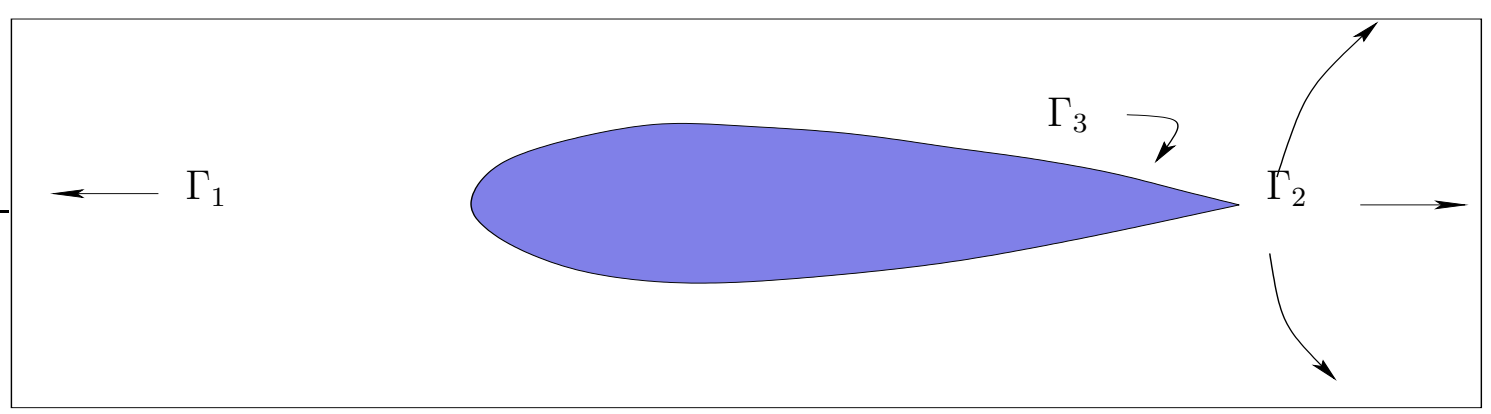

Figura 2.3: Domínio computacional: geometria do aerofólio e contornos. 
A velocidade das gotículas, bem como o conteúdo de água líquida, é imposto para a corrente livre. A velocidade da gotícula é igual a zero para todas as paredes para o início dos cálculos. Não é necessário as condições de contorno para a saída, devido à natureza hiperbólica do sistema de equações.

$\Gamma_{1}$ : Contorno da entrada

Responsável pela água que entra no domínio, assim assume-se a condição de contorno de Dirichlet para o domínio:

$$
\begin{aligned}
& \left.\alpha\right|_{\Gamma_{1}} \equiv \alpha_{\infty}, \text { onde } \alpha_{\infty} \text { é um valor constante. } \\
& \left.V_{d}\right|_{\Gamma_{1}} \equiv V_{a} .
\end{aligned}
$$

$\Gamma_{2}$ : Contorno da saída

A condição estabelece que o gradiente é zero na saída (condição de contorno de Neumann). Assim,

$$
\left.\frac{\partial U}{\partial n}\right|_{\Gamma_{2}} \equiv 0
$$

onde $\mathrm{U}=\left(\alpha, u_{d}, v_{d}\right)$.

$\Gamma_{3}$ : Contorno do aerofólio

No contorno do aerofólio, apartir de um ponto tem-se a região de sombra, onde $\hat{U}_{d}=0$. Para definir quais pontos do contorno fazem parte desta região, estabelecese um threshold (percentual mínimo) da velocidade e analisa-se:

Caso 1: $\epsilon_{V}$ é o threshold da velocidade

Se $V_{d}=\left\|V_{d}\right\|=\left\|\left(u_{d}, v_{d}\right)^{T}\right\|<\epsilon_{V}$, então $\hat{U}_{d}=0$, onde $V_{d}$ é a velocidade da gotícula.

Caso 2: Se $V_{d}>\epsilon_{V}$, calcula-se a direção do fluxo e o próprio fluxo

$$
V_{d i r}=\frac{V_{d} \cdot n}{\left\|V_{d}\right\|\|n\|}
$$

Se $V_{d} \leq \epsilon_{V}$, define-se um threshold para $V_{d i r}\left(\epsilon_{n}\right)$ e, caso $V_{d i r}<\epsilon_{n}$ (se a velocidade da gotícula é quase perpendicular à $\mathrm{n})$, então $\hat{U}=0$. 
Se $V_{d}>\epsilon_{V}$ então estabelece-se que o gradiente é zero

$$
\left.\frac{\partial U}{\partial n}\right|_{\Gamma_{3}} \equiv 0
$$

onde $U=\left(\alpha, u_{d}, v_{d}\right)$

\subsubsection{Proposta do presente trabalho}

A formulação euleriana ainda vem ganhando popularidade na indústria aeroespacial uma vez que elimina a dificuldade da semeadura de gotículas, característica específica da formulação lagrangiana, mas é limitado para um tamanho de gotícula por fase, resultando em computação adicional quando alguns tamanhos diferentes de gotículas precisam ser analisados, adicionando um significativo tempo computacional.

A aplicação da formulação lagrangiana é complicada para geometrias complexas pois diferentes locais de semeadura são necessários para os locais de impacto das partículas em um corpo. Quanto à formulação euleriana, como ela resolve a equação das gotículas como uma fase contínua não precisa de uma emissão de semeadura. Ela pode ser implementada de modo idêntido às equações de Navier-Stokes usando malhas não-estruturadas que são capazes de suportar geometrias complexas.

Assim, o cálculo da eficiência de coleta contornando todo o corpo pode ser obtida em um único cálculo enquanto que na formulação lagrangiana não é prático para determinar os contornos da coleta sobre todo o corpo investigado uma vez que ele requer um número impraticável de gotículas.

A formulação euleriana, como toda equação de transporte de um escalar tem uma dissipação artificial que pode mascarar os resultados físicos [58]. Uma solução para isto é o refinamento da malha ou então a utilização de um método de alta ordem para capturar os efeitos na região de impacto.

Ainda, o cálculo de $\alpha$, fração de volume de água, nos estudos desenvolvidos até o momento, [42] e [73], utilizam esquemas de dissipação artificial que podem influenciar consideravelmente no cálculo dos limites de impacto.

Desta forma, este estudo se propõe a produzir um novo esquema de alta ordem costumizado para o cálculo do coeficiente de coleta dos apresentados na literatura, como mostra a tabela comparativa Tabela 2.1 . 


\begin{tabular}{|c|c|c|c|c|c|c|}
\hline \multirow[t]{2}{*}{ Código } & \multirow[t]{2}{*}{$\begin{array}{l}\text { Modelo do } \\
\text { escoamento }\end{array}$} & \multirow[t]{2}{*}{ Malha } & \multicolumn{2}{|c|}{$\begin{array}{c}\text { Ordem da } \\
\text { deiscretização }\end{array}$} & \multirow[t]{2}{*}{$\begin{array}{l}\text { Formulação } \\
\text { de partículas }\end{array}$} & \multirow[t]{2}{*}{ Origem } \\
\hline & & & Espacial & Temporal & & \\
\hline LEWICE & Potencial + CL & Contorno & $1^{\mathrm{a}}$ & $1^{\mathrm{a}}$ & lagrangiana & $\begin{array}{l}\text { NASA } \\
\text { (EUA) }\end{array}$ \\
\hline ONERA & Potencial + CL & Contorno & $1^{\mathrm{a}}$ & $1^{\mathrm{a}}$ & lagrangiana & $\begin{array}{l}\text { ONERA } \\
\text { (França) }\end{array}$ \\
\hline TRAJICE2 & Potencial + CL & Contorno & $1^{\mathrm{a}}$ & $1^{\mathrm{a} .}$ & lagrangiana & $\begin{array}{c}\text { DERA } \\
(\mathrm{GB})\end{array}$ \\
\hline CANICE & Potencial + CL & Contorno & $1^{\mathrm{a}}$ & $1^{\mathrm{a}}$ & lagrangiana & $\begin{array}{c}\text { NRC } \\
\text { (Canadá) }\end{array}$ \\
\hline CIRA & Potencial + CL & Contorno & $1^{\mathrm{a}}$ & $1^{\mathrm{a}}$ & lagrangiana & $\begin{array}{l}\text { CIRA } \\
\text { (Itália) }\end{array}$ \\
\hline AEROICING & Potencial + CL & Contorno & $1^{\mathrm{a}}$ & $1^{\mathrm{a}}$ & lagrangiana & $\begin{array}{c}\text { UFSC } \\
\text { (Brasil) }\end{array}$ \\
\hline FENSAP-ICE & Navier-Stokes & Não-estruturada & $2^{\mathrm{a}}$ & $2^{\mathrm{a}}$ & euleriana & $\begin{array}{l}\text { Habashi } \\
\text { (Canadá) }\end{array}$ \\
\hline FLUENT & Navier-Stokes & Não-estruturada & $2^{\mathrm{a}}$ & $2^{\mathrm{a}}$ & euleriana & $\begin{array}{l}\text { ANSYS } \\
(\text { EUA) }\end{array}$ \\
\hline $\begin{array}{l}\text { Presente } \\
\text { trabalho }\end{array}$ & Navier-Stokes & Não-estruturada & $\geq 2^{\mathrm{a}}$ & $\geq 2^{\mathrm{a}}$ & euleriana & $\begin{array}{c}\text { USP } \\
\text { (Brasil) }\end{array}$ \\
\hline
\end{tabular}

CL: camada limite

Tabela 2.1: Formulações para o cálculo do coeficiente de coleta. 
Capítulo 3

\section{Modelagem do escoamento}

Este capítulo apresenta o método dos volumes finitos em malhas não-estruturadas triangulares; a escolha do domínio e a geração da malha, bem como o tratamento adotado para a obtenção do escoamento.

\subsection{Método dos volumes finitos}

O conceito chave usado durante toda a formulação de volumes finitos é o princípio de conservação de uma determinada quantidade física expressa pelas equações governantes sobre qualquer volume, também denominado volume de controle (VC).

O domínio do escoamento é discretizado em um conjunto de volumes de controle não sobrepostos, que podem ser irregulares no tamanho e na forma.

O uso de malhas não-estruturadas é justificado pela facilidade de adaptação destas malhas para geometrias complexas, permitindo que refinamentos locais possam ser feitos em regiões específicas.

Os valores de uma variável dependente podem ser armazenados no centróide do volume de controle (cell-centered) ou então em um vértice comum aos elementos vizinhos, (cellvertex), Figura 3.1. 


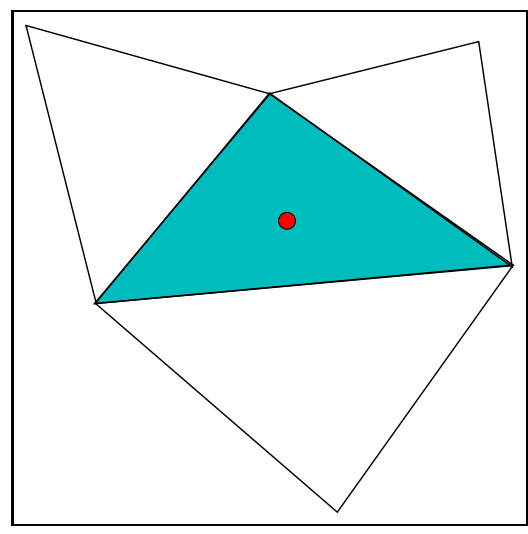

(a)

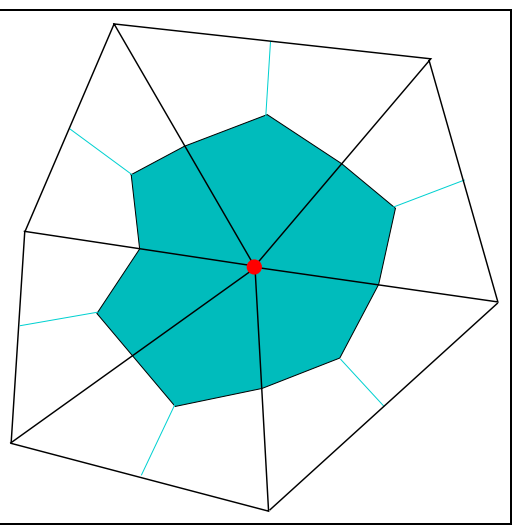

(b)

Figura 3.1: Volume de controle em uma malha não-estruturada: (a) centrado na célula; (b) centrado no vértice [70].

As equações discretizadas da variável dependente são obtidas integrando a equação governante sobre cada um dos volumes de controle no domínio. O processo de discretização torna-se mais conveniente reconhecendo-se o fato que todas as equações governantes relevantes possuem uma forma comum, isto é, a forma da equação geral em 2D

$$
\frac{\partial \phi(x, y)}{\partial t}+\frac{\partial F(x, y)}{\partial x}+\frac{\partial G(x, y)}{\partial y}=S(x, y)
$$

sendo $\phi$ é o vetor solução, $\mathrm{S}$ é o termo fonte e $F$ e $G$ são os termos do fluxo. A forma volume finito pode ser escrita como:

$$
\frac{\partial \bar{\phi}_{i}}{\partial t}+\frac{1}{V_{i}} \oint(F \vec{i}+G \vec{j}) \cdot \vec{n} d A-\bar{S}=0
$$

onde

$V_{i}$ é a área do volume de controle i;

A é um comprimento;

n é a normal apontando para fora do volume de controle.

\subsection{Definição da geometria e geração da malha com- putacional}

Pela Figura 3.2, uma das etapas da resolução do problema de transporte de gotículas é o tratamento do escoamento. Anteriormente a isto, tem-se a definição da geometria 
que é o primeiro passo para desenvolvimento da solução de um problema por um método computacional. Determinada a geometria do problema, suas informações são transferidas para um gerador de malha.

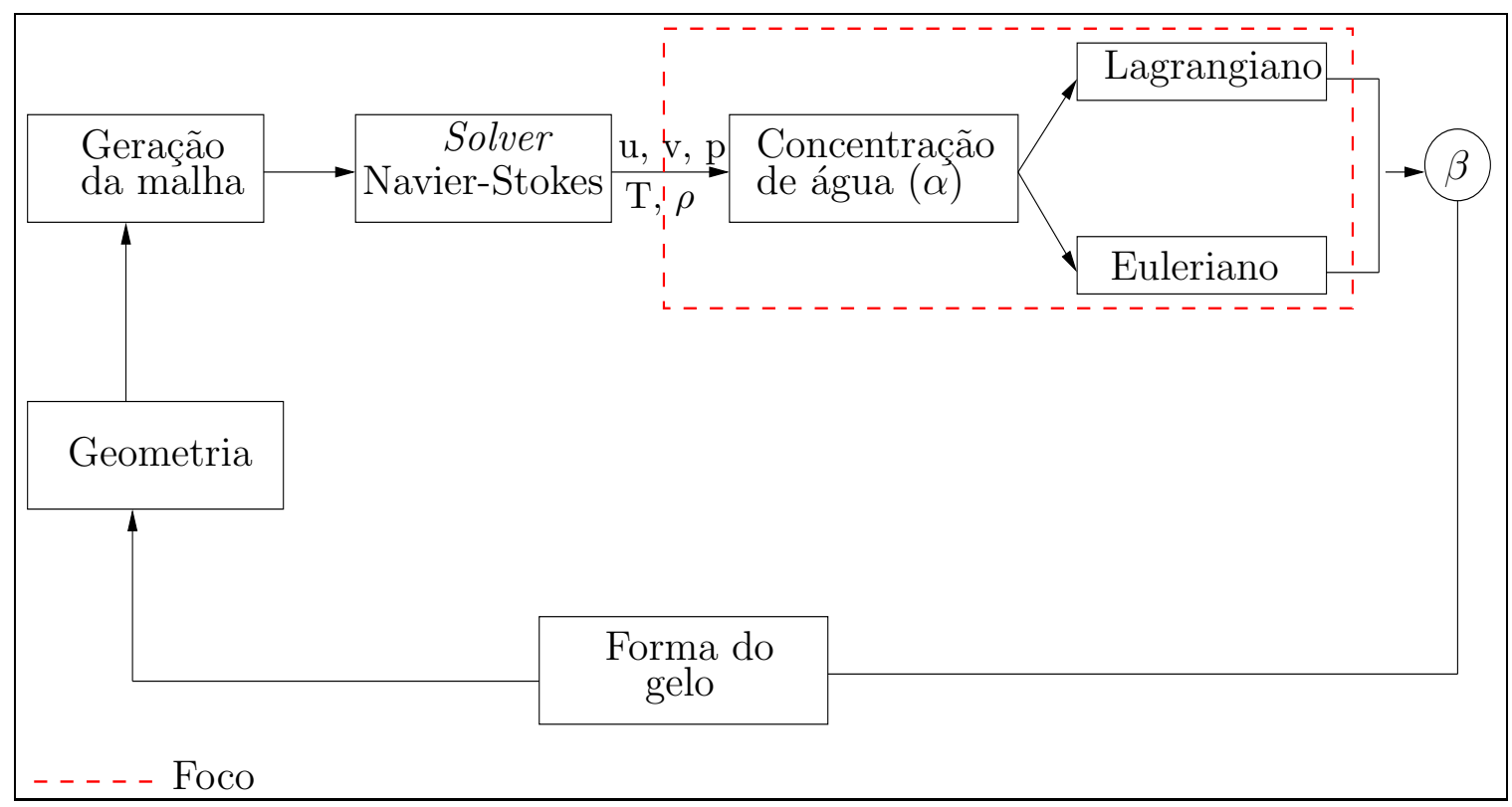

Figura 3.2: - - -Foco: construir esquemas numéricos para a solução das equações de transporte na formulação euleriana.

O gerador de malhas adotado neste trabalho é o EMC2 [56], de uso livre, que foi desenvolvido no INRIA - L' Institut National de Recherche en Informatique et en Automatique da França [85].

O EMC2 - Edition de Maillages et de Contours en 2 Dimensions, desenvolvido por Frédéric Hecht e Eric Saltel, é um gerador de geometrias e malhas em duas dimensões de forma portátil e iterativa; pode-se criar e modificar uma geometria usando o CAD Computer Aided Design, definir as discretizações sobre as linhas, definir os subdomínios e definir alguns números de referência que levam em conta as condições de contorno e as propriedades materiais. O modelo do editor de malhas EMC2 é apresentado na Figura 3.3.

Os elementos da malha podem ser triângulos e quadriláteros e há dois tipos de malhas: por grades e Delaunay-Voronoi, que é a malha automática (default). Estas malhas podem ser re-editadas usando as várias funções, por exemplo, movendo, removendo, adicionando 
vértices por simetria ou rotação.

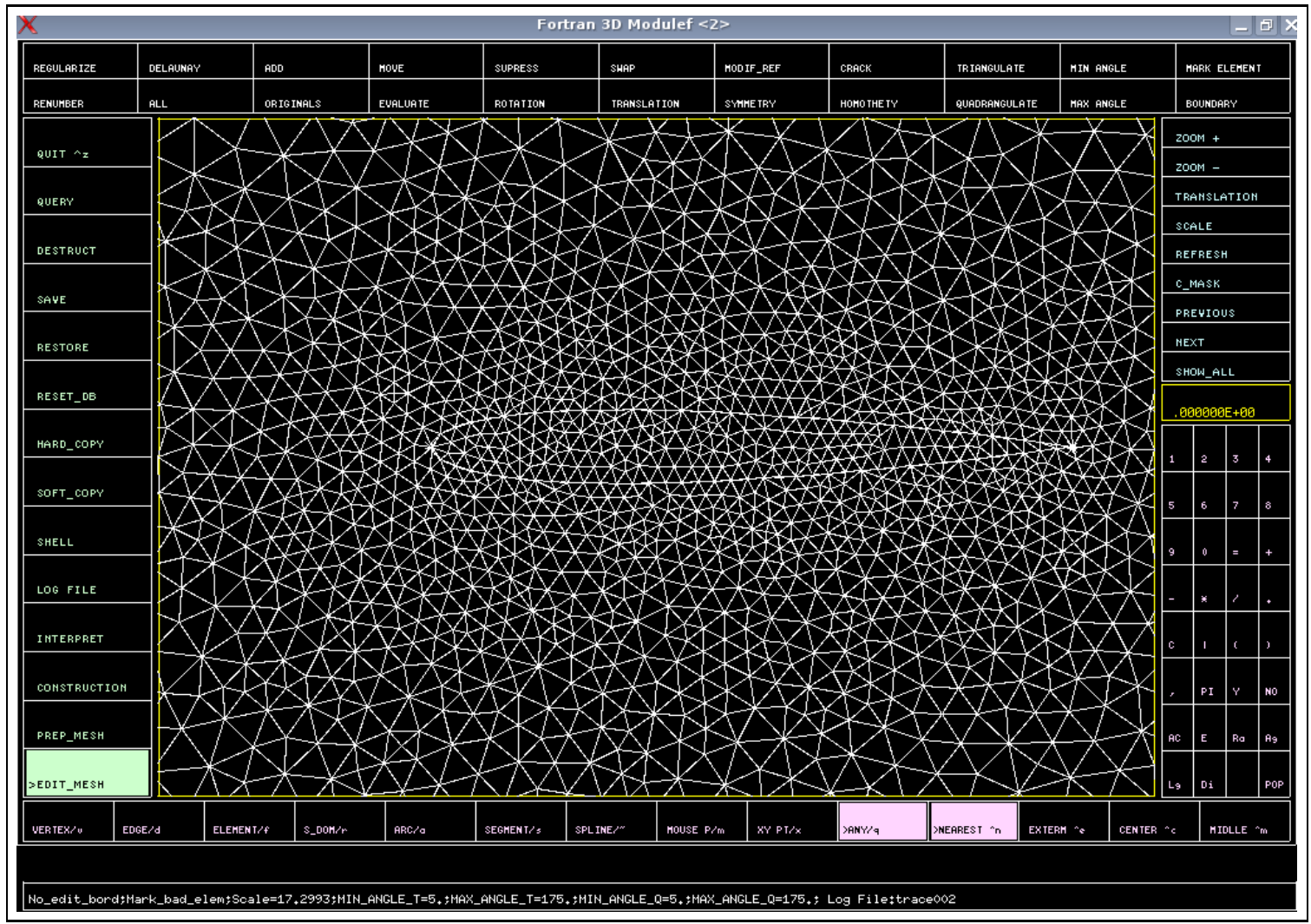

Figura 3.3: Editor de malhas EMC2 [56].

O EMC2 permite gerar arquivos com vários formatos, dentre estes formatos a escolha natural foi o amdba, usada pelo NSC2KE e adotado neste trabalho para a construção de uma estrutura de dados a ser aproveitada pelo solver do escalar $\phi$ no transporte de gotículas.

Um arquivo com extensão amdba gerado pelo EMC2 tem o formato apresentado na Tabela 3.1, sendo: nv é o total de vértices na malha;

nt é o total de triângulos na malha;

$v_{i}$ é o vértice $i, \mathrm{i}=1, \ldots, \mathrm{nv}$;

$x_{i}$ e $y_{i}$ são as coordenadas do vértice $i$;

$p v_{i}$ é uma propriedade do vértice $i$;

$t_{j}$ é o triângulo $j, \mathrm{j}=1, \ldots, \mathrm{nt}$;

$v t_{j 1}, v t_{j 2}, v t_{j 3}$ são os vértices do triângulo $j$. 


\begin{tabular}{|ccccc|}
\hline $\mathrm{nv}$ & $\mathrm{nt}$ & & & \\
$v_{1}$ & $x_{1}$ & $y_{1}$ & $p v_{1}$ & \\
$v_{2}$ & $x_{2}$ & $y_{2}$ & $p v_{2}$ & \\
$\vdots$ & $\vdots$ & $\vdots$ & $\vdots$ & \\
$v_{n v}$ & $x_{n v}$ & $y_{n v}$ & $p v_{n v}$ & \\
$t_{1}$ & $v t_{11}$ & $v t_{21}$ & $v t_{31}$ & $p t_{1}$ \\
$t_{2}$ & $v t_{12}$ & $v t_{22}$ & $v t_{32}$ & $p t_{2}$ \\
$\vdots$ & $\vdots$ & $\vdots$ & $\vdots$ & $\vdots$ \\
$t_{n t}$ & $v t_{n t}$ & $v t_{n t}$ & $v t_{n t}$ & $p t_{n t}$ \\
\hline
\end{tabular}

Tabela 3.1: Formato do arquivo amdba obtido através do EMC2.

Uma vez gerado o arquivo de dados, faz-se o tratamento do escoamento, resolvendo as equações de Navier-Stokes.

\subsection{Solução numérica das equações de Navier-Stokes}

Para a solução numérica das equações que governam o escoamento do ar é usado o solver NSC2KE [10, 41] - Navier-Stokes Compressible 2D k-€ Model, que é de uso livre e foi desenvolvido pelo INRIA [85]. O NSC2KE usa uma combinação dos métodos de volumes finitos e elementos finitos para simular uma larga extensão de campos de escoamentos indo do subsônico para o regime hipersônico para configurações axissimétricas e em duas dimensões.

Como configurações reais às vezes envolvem geometrias complexas, a malha nãoestruturada tem se mostrado uma boa ferramenta para uma melhor aproximação da realidade e é usada neste código.

O código foi desenvolvido em Fortran77 por Bijan Mohammadi e resolve tanto as equações de Euler quanto Navier-Stokes, usando o esquema upwind e método de interpolação linear para o cálculo dos fluxos convectivos em uma formulação em volumes finitos, o Método Clássico de Galerkin Central P1-Elementos Finitos para a computação dos fluxos difusivos e o modelo de turbulência k- $\epsilon$ para resolver escoamentos turbulentos. Para 
calcular a turbulência próximo da parede é usada a aproximação em duas camadas ou as leis de parede.

O único pré-processamento requerido é definir uma malha e o EMC2 é uma escolha natural no pré-processamento do tratamento de um escoamento através do solver NSC2KE e servirá de base para a solução do escoamento do ar, obtendo o campo de velocidade a ser utilizado para o transporte de gotículas em uma formulação euleriana.

O NSC2KE gera gráficos em uma ou duas dimensões que podem ser diretamente visualizados usando a conhecida ferramenta gráfica GNUPLOT. Neste trabalho adaptouse a saída gráfica para usar o aplicativo TECPLOT [89].

As classes de problemas que o NSC2KE podem simular envolve uma larga quantidade de campos de escoamentos axissimétricos em duas dimensões, incluindo:

- escoamentos internos e externos;

- escoamentos subsônicos para invíscido hipersônico: o código tem sido testado para números de Mach entre 0.1 e 10;

- escoamentos subsônicos a baixo Reynolds para escoamentos viscosos hipersônicos;

- escoamentos turbulentos, do subsônico ao hipersônico turubulento completamente separado;

- escoamentos em regime permanente ou não.

Para definir a configuração computacional, o usuário deve definir duas quantidades adimensionais, denominadas de número de Reynolds e número de Mach que descreverão o campo do escoamento, arquivo DATA.

Pelo arquivo DATA o usuário pode escolher o tipo de solver que deseja usar, por exemplo, para a parte das equações de Euler foram escritos os seguintes solvers: Roe, Osher e cinético enquanto que para a parte viscosa somente um esquema centrado é avaliado. Não há regras para a escolha do solver convectivo. Para escoamentos subsônico ou levemente transônico todos os três solvers podem ser usados. Mas com o crescimento do número de Mach, o solver Roe não será robusto o suficiente. Para entender melhor os parâmetros requeridos pelo código alguns casos testes tem sido reproduzidos no final do manual. 
O NSC2KE propõe esquemas com precisão de primeira e segunda ordem espacial. O usuário deve escolher a precisão do esquema através do parâmetro node:

- node $=1$ : esquema de primeira ordem;

- node $=2$ : esquema de segunda ordem;

- node $=3$ : esquema de segunda ordem com Limitante Van Albada.

Para a implementação das condições de contorno, cada nó possui um índice, um parâmetro inteiro, denominado logic. Assim, logic igual a

$\mathbf{0}$ denota um nó interno;

2 denota uma simetria ou um ponto slipping;

3 denota um ponto do contorno sólido;

4 denota um ponto de saída do escoamento;

5 denota um ponto do contorno de entrada;

6 denota um ponto de entrada forçada.

Aplica-se o Método dos Volumes Finitos-Galerkin para a parte convectiva e o método de Galerkin Padrão para a parte viscosa.

A formulação global é dada por

$$
\frac{\partial W}{\partial t}+\nabla \cdot(F(W)-N(W))=0
$$

onde

$$
W=\left(\rho, \rho u_{1}, \rho u_{2}, \rho E, \rho K, \rho \epsilon\right)^{t} .
$$

O método consiste em encontrar $W_{h} \in\left(V_{h}\right)$ tal que $\forall \phi_{h} \in V_{h}$ e

$$
\int_{\Omega} \frac{\partial W_{h}}{\partial t} \Phi_{h} d \Omega-\int_{\Omega}\left(F_{h}-N_{h}\right)\left(W_{h}\right) \nabla \phi_{h} d \Omega+\int_{\partial \omega}\left(F_{h}-N_{h}\right) \cdot n \phi_{h} d \omega=0 .
$$

Utilizando uma integração temporal explícita, pela Figura 3.4, tem-se

$$
\left|C_{i}\right| \frac{W^{n+1}-W^{n}}{\Delta t}+\sum_{j \neq i} \Phi\left(W_{i}^{\prime}, W_{j}^{\prime}\right) \int_{\partial C_{i} \cap C_{j}} n d \Gamma=R H S
$$




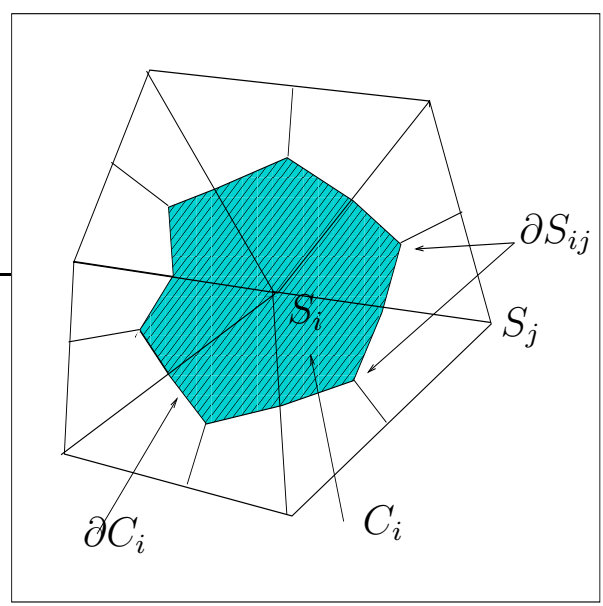

Figura 3.4: Volume de controle para a discretização espacial: Método dos Volumes Finitos Galerkin.

onde o RHS é a parte difusiva dada por

$$
R H S=-\int_{\Omega_{h}} N\left(W^{N}\right) \nabla \phi_{h} d \Omega+\int_{\partial \Omega} N\left(W^{N}\right) \cdot \phi_{h} n d \Gamma .
$$

$\mathrm{e}$

$$
\Phi(u, v)=0.5[F(u)+F(v)]-d(u, v)
$$

A precisão de segunda ordem é obtida usando um MUSCL envolvendo a combinação de upwind e gradientes centrados. Mais precisamente, faz-se $\nabla W_{i}$ ser uma aproximaçãoo do gradiente de $\mathrm{W}$ para o nó i, em seguida denota-se o segmento $\overline{i j}$ como

$$
\left\{\begin{array}{l}
W_{i j}=W_{i}+0.5 \lim \left(\beta(\nabla W)_{i}\left(\overrightarrow{i j},(1-\beta)\left(W_{i}-W_{j}\right)\right)\right. \\
W_{j i}=W_{j}-0.5 \lim \left(\beta(\nabla W)_{j}\left(\overrightarrow{i j},(1-\beta)\left(W_{j}-W_{i}\right)\right)\right.
\end{array}\right.
$$

onde, o Limitador de Van Albada é dado por

$$
\lim (a, b)=0.5(1+\operatorname{sinal}(a b)) \frac{\left(a^{2}+\alpha\right) b+\left(b^{2}+\alpha\right) a}{a^{2}+b^{2}+2 \alpha}, \quad 0<\alpha<<1
$$

Para a integração temporal o NSC2KE utiliza um método explícito, Runge-Kutta 4-Estágios [12, 38].

Reescrevendo 3.5 como

$$
\frac{\partial W}{\partial t}=R H S(W) .
$$


Dá-se início ao processo iterativo, fazendo

$$
\left\{\begin{array}{c}
W^{0}=W^{n} \\
W^{k}=W^{o}+\alpha_{k} \Delta t R H S\left(W^{k-1}\right), \mathrm{k}=1, \cdots, 4 . \\
W^{k+1}=W^{4}
\end{array}\right.
$$

onde a escolha de $\alpha_{k}$ em [38] é dada por:

$$
\alpha_{1}=0.11 \quad \alpha_{2}=0.2766 \quad \alpha_{3}=0.45 \quad \alpha_{4}=1.0 .
$$

Os parâmetros $\alpha_{k}$ são os valores ótimos para o esquema e forma propostos por M. H. Lallemand [38].

$$
\Delta t_{i}=\min \left(\frac{\Delta x}{|u|+c}, \frac{\rho \operatorname{Pr} \Delta x^{2}}{2\left(\mu+\mu_{t}\right)}\right)
$$

onde $\Delta x$ é a altura mínima de todos os triângulos que tem em comum o vértice i.

\subsubsection{Campo de velocidade na região retangular com cilindro em 2D}

O domínio a ser estudado é uma região retangular de dimensões $\Omega=[-2 D, 6 D] \times$ $[-3 D, 3 D]$, com $D=0.1016 \mathrm{~m}$ diâmetro do cilindro da Figura 3.5, baseado em [73].

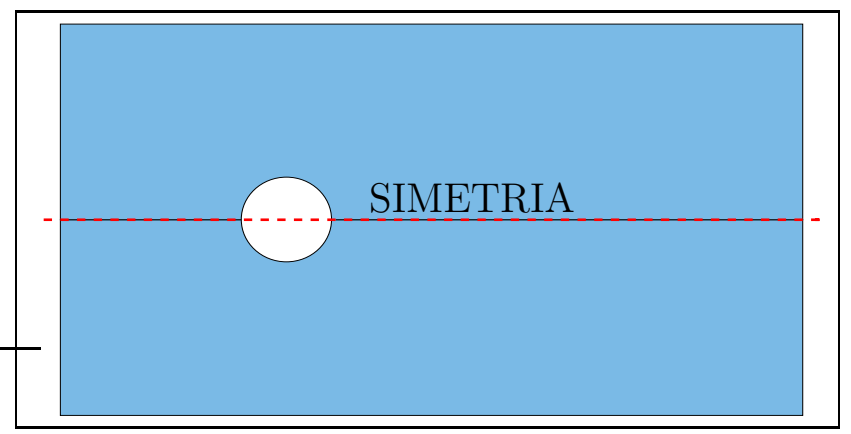

Figura 3.5: Domínio a ser estudado: cilindro de diâmetro $D=0.1016 \mathrm{~m}$ em uma região retangular $\Omega=[-2 D, 6 D] \times[-3 D, 3 D]$.

Devido à simetria, nos testes será utilizado apenas a região contendo o semi-cilindro e então será feito um rebatimento no eixo de simetria para a apresentação dos resultados. 
Para determinar os parâmetros do arquivo DATA é usado a Lei de Sutherland. Para o coeficiente de viscosidade molecular a Lei de Sutherland é dada por

$$
\mu=\mu_{\text {ref }}\left(\frac{T}{T_{\text {ref }}} \frac{T_{r e f}+T_{s}}{T+T_{s}}\right)
$$

onde

$T$ é a temperatura em Kelvin;

$T_{s}$ é a constante de Sutheland para o material gasoso em questão;

$T_{\text {ref }}$ é a temperatura de referência em Kelvin.

Para o ar, as variáveis assumem os seguintes valores:

$T_{s}=110.6 K$

$T_{\text {ref }}=273.15 \mathrm{~K}$

$\mu_{\text {ref }}=1.716 \times 10^{-5}$

Assim, os dados de entrada no solver são fornecidos pelo arquivo DATA e consta de:

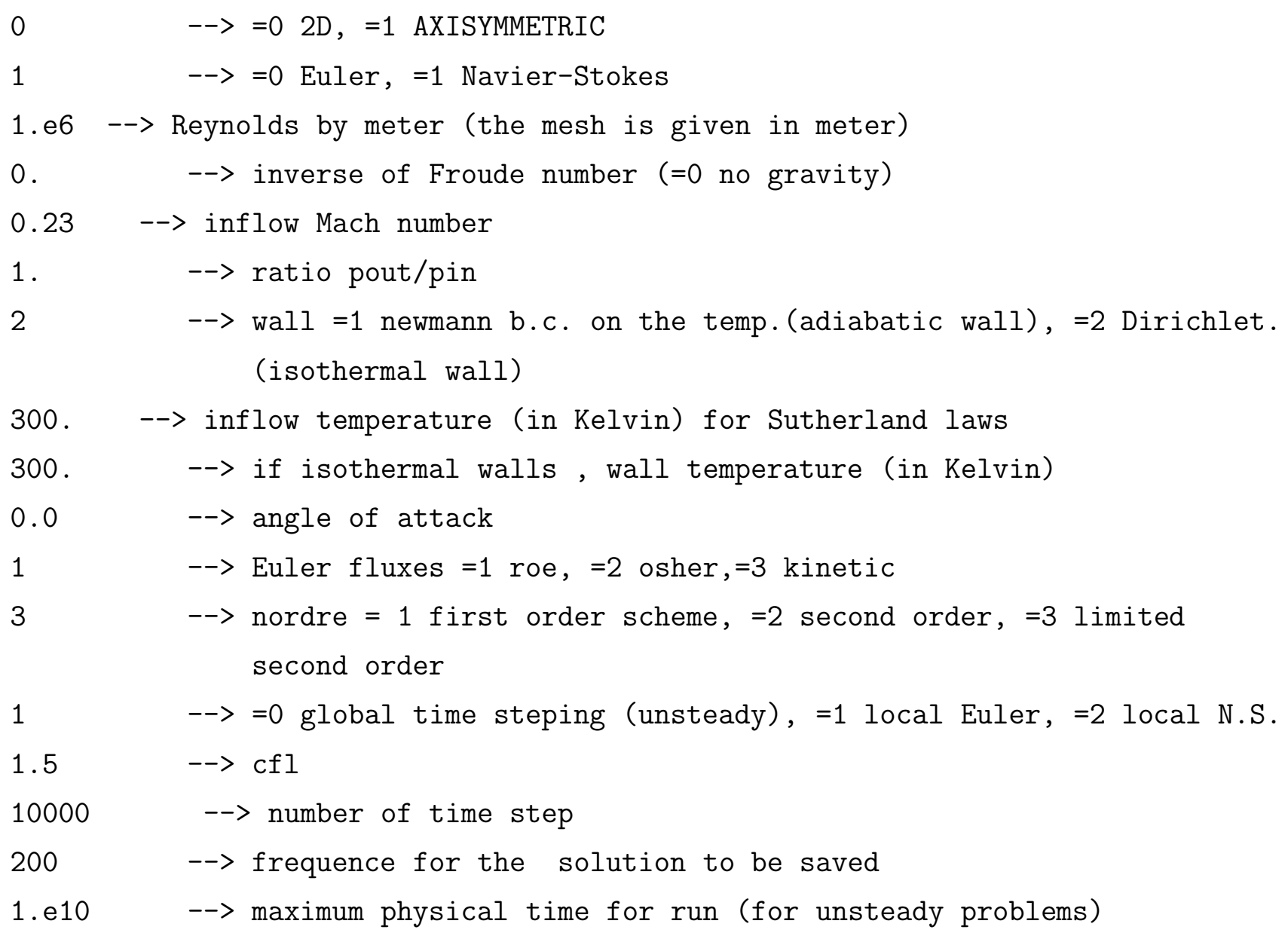




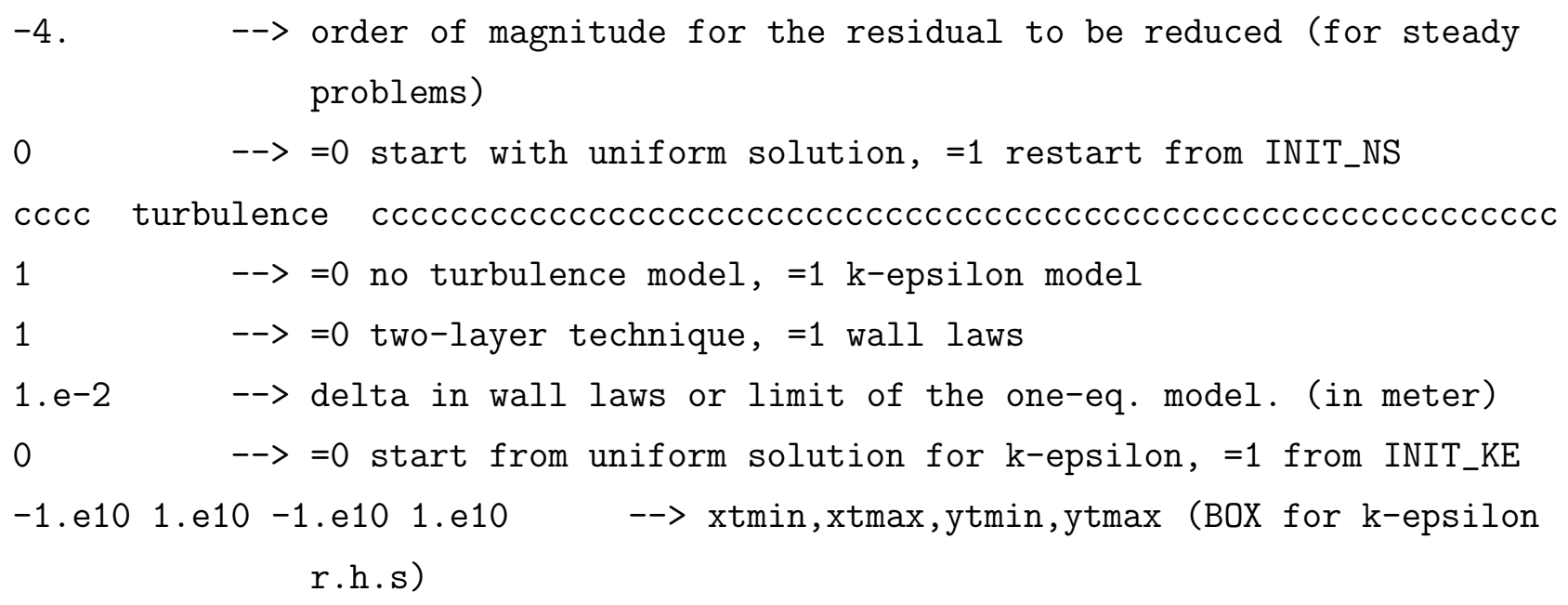

Outros dados são:

$D=0.1016 m$

$p=89867 \mathrm{~Pa}$

$V_{\infty}=80 \mathrm{~m} / \mathrm{s}$

$\rho=1.097 \mathrm{~kg} / \mathrm{m}^{3}$

$M_{\infty}=V_{\infty} / a_{\infty}$

$R e=\frac{\rho_{\infty} v_{\infty} d}{\mu_{\infty}}$

$\mathrm{O}$ valor de $\delta$ (delta in wall laws or limit of the one-eq. model. (in meter)) varia de acordo com o número de partições no cilindro

$$
\delta=\pi r n
$$

onde r é o raio do cilindro e n o número de partições sobre ele.

Os valores dos parâmetros utilizados buscam produzir diferentes campos de velocidade e regiões com vórtices para analisar a sua influência no transporte de um escalar $\phi$.

As seguintes condições de contorno apresentadas na Figura 3.6. Cada segmento e o cilindro da Figura 3.6 é apresentado na Figura 3.7 pelas letras A, B, ..., F. A Tabela 3.2 apresenta o número de partições em cada segmento e no cilindro nas malhas m1, m2, ..., m6 que serão utilizadas nos testes. 


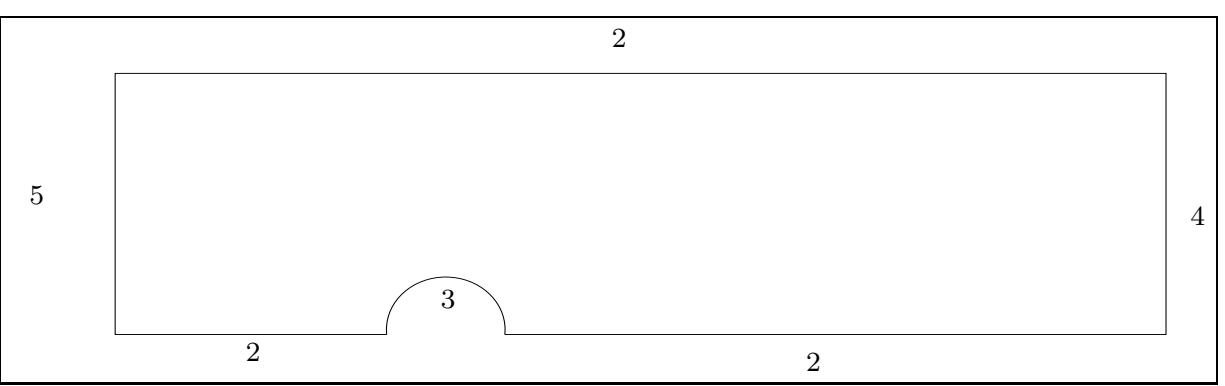

Figura 3.6: Condição de contorno para o escoamento do ar: $2=\operatorname{simetria;} 3=$ sólido; $4=$ saída e $5=$ entrada.

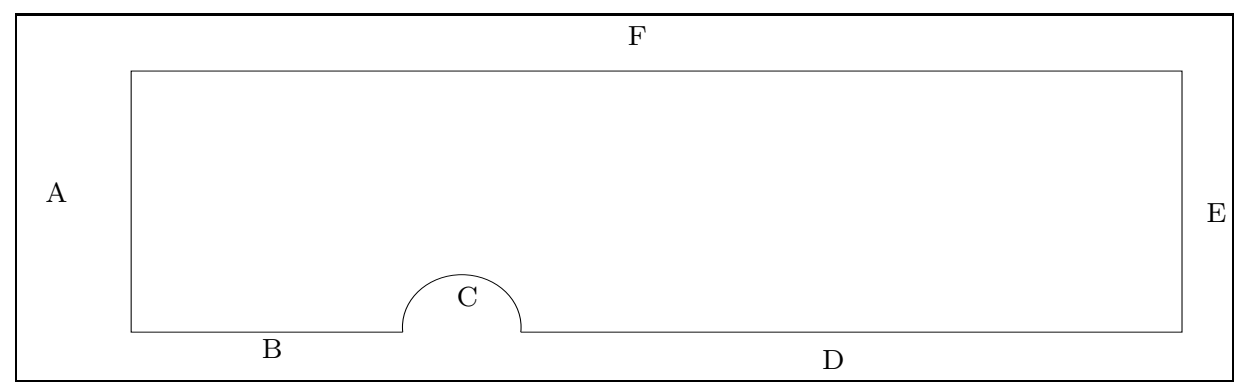

Figura 3.7: Nomenclatura dos contornos do domínio para a definição do número de partições.

O número de vértices, triângulos e faces é apresentando na Tabela 3.3, bem como o tempo de execução do solver NSC2KE, executado no LCCA.

\begin{tabular}{|c|c|c|c|c|c|c|}
\hline segmento/cilindro & $m 1$ & m2 & $m 3$ & $m 4$ & $m 5$ & $m 6$ \\
\hline A & 28 & 56 & 112 & 28 & 56 & 112 \\
\hline B & 28 & 56 & 112 & 28 & 56 & 112 \\
\hline C & 28 & 56 & 112 & 28 & 56 & 112 \\
\hline D & 56 & 112 & 224 & 112 & 224 & 448 \\
\hline E & 56 & 112 & 224 & 112 & 224 & 448 \\
\hline F & 56 & 112 & 224 & 56 & 112 & 224 \\
\hline
\end{tabular}

Tabela 3.2: Número de partições em cada segmento/cilindro do domínio apresentado na Figura 3.7. 


\begin{tabular}{|c|c|c|c|c|}
\hline malha & no. de vértices & no. de triângulos & número de arestas & tempo (s) \\
\hline $\mathrm{m} 1$ & 3559 & 6864 & 10422 & 488 \\
\hline $\mathrm{m} 2$ & 13664 & 26822 & 40485 & 4044 \\
\hline $\mathrm{m} 3$ & 55239 & 109468 & 164706 & 17439 \\
\hline $\mathrm{m} 4$ & 6724 & 13082 & 19805 & 1174 \\
\hline $\mathrm{m} 5$ & 26866 & 53002 & 79867 & 8980 \\
\hline $\mathrm{m} 6$ & 76013 & 150792 & 226804 & 28329 \\
\hline
\end{tabular}

Tabela 3.3: Número de elementos em cada malha, com número de partições dado pela Tabela 3.2 e respectivo tempo de execução para $R e=1.0 \times 10^{6}$.

Um zoom das malhas da Tabela 3.3 são apresentadas nas Figuras 3.8 - 3.13.

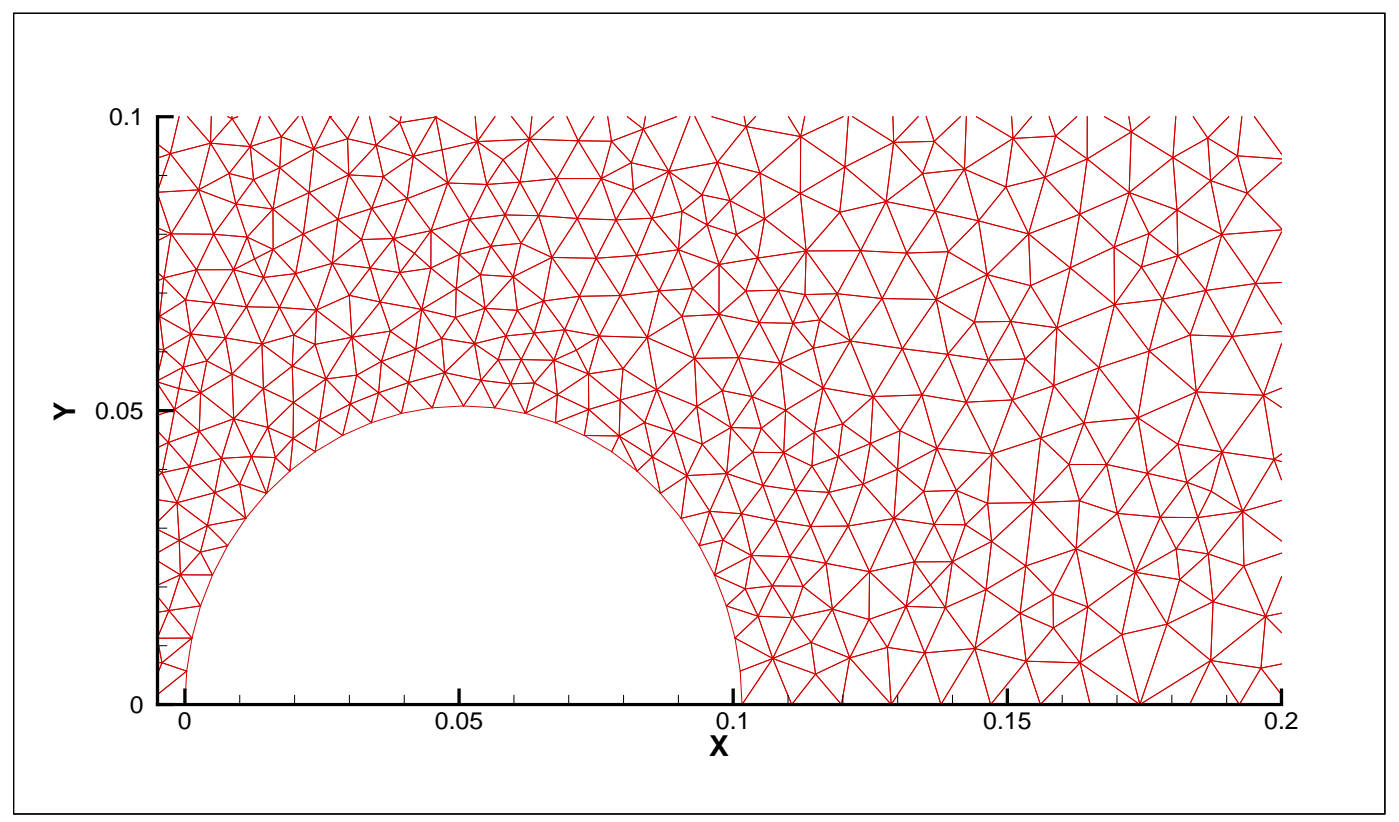

Figura 3.8: zoom da malha $m 1$. 


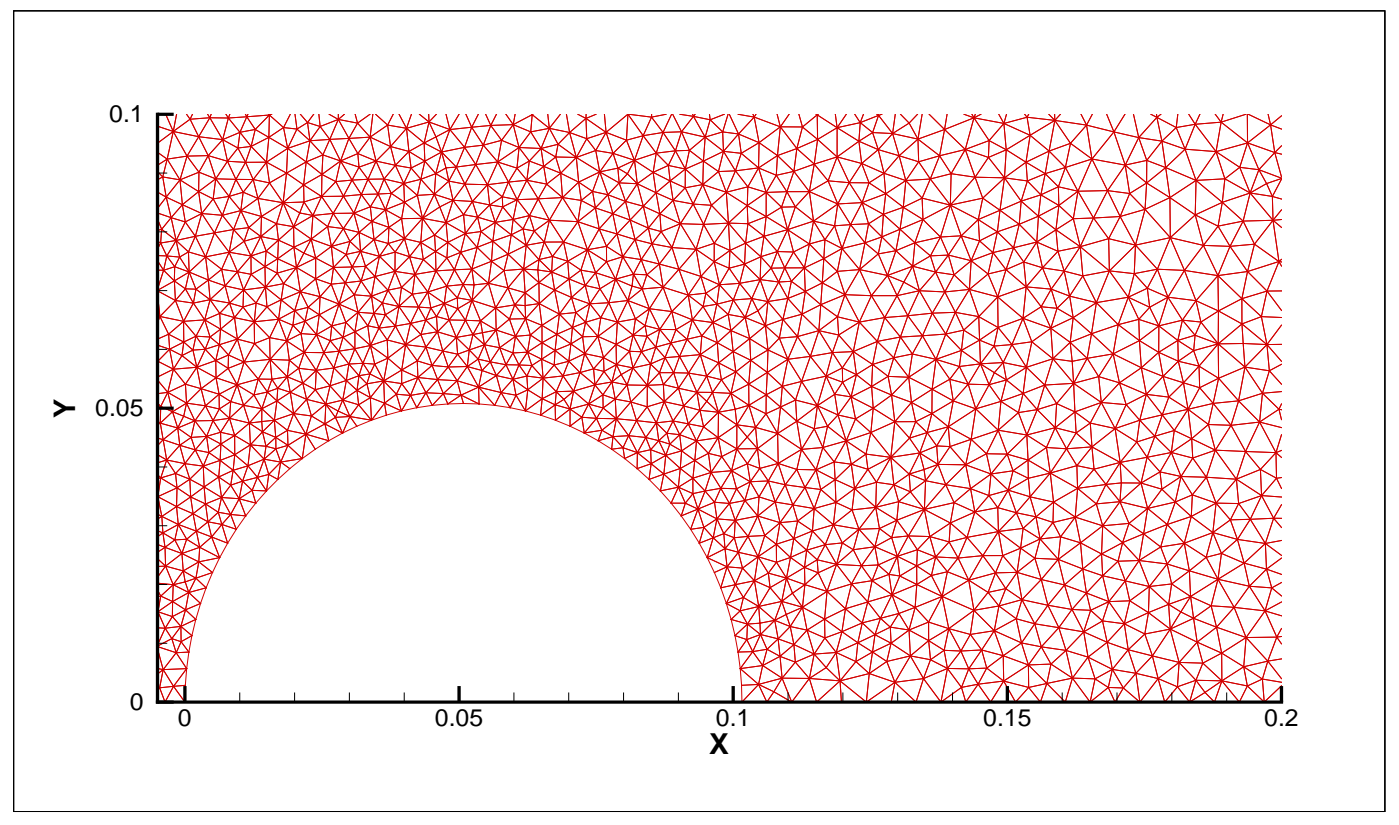

Figura 3.9: zoom da malha m2.

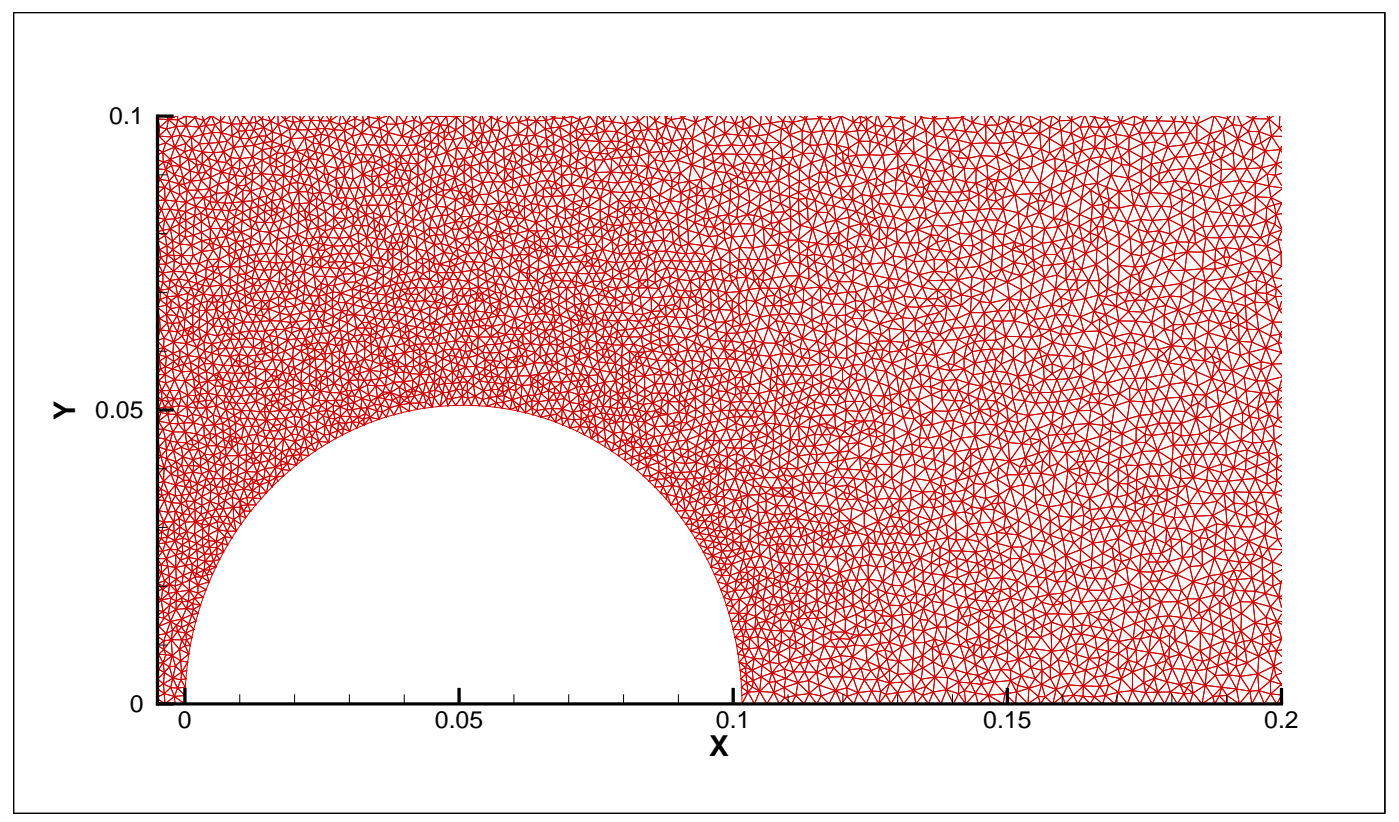

Figura 3.10: zoom da malha m3. 


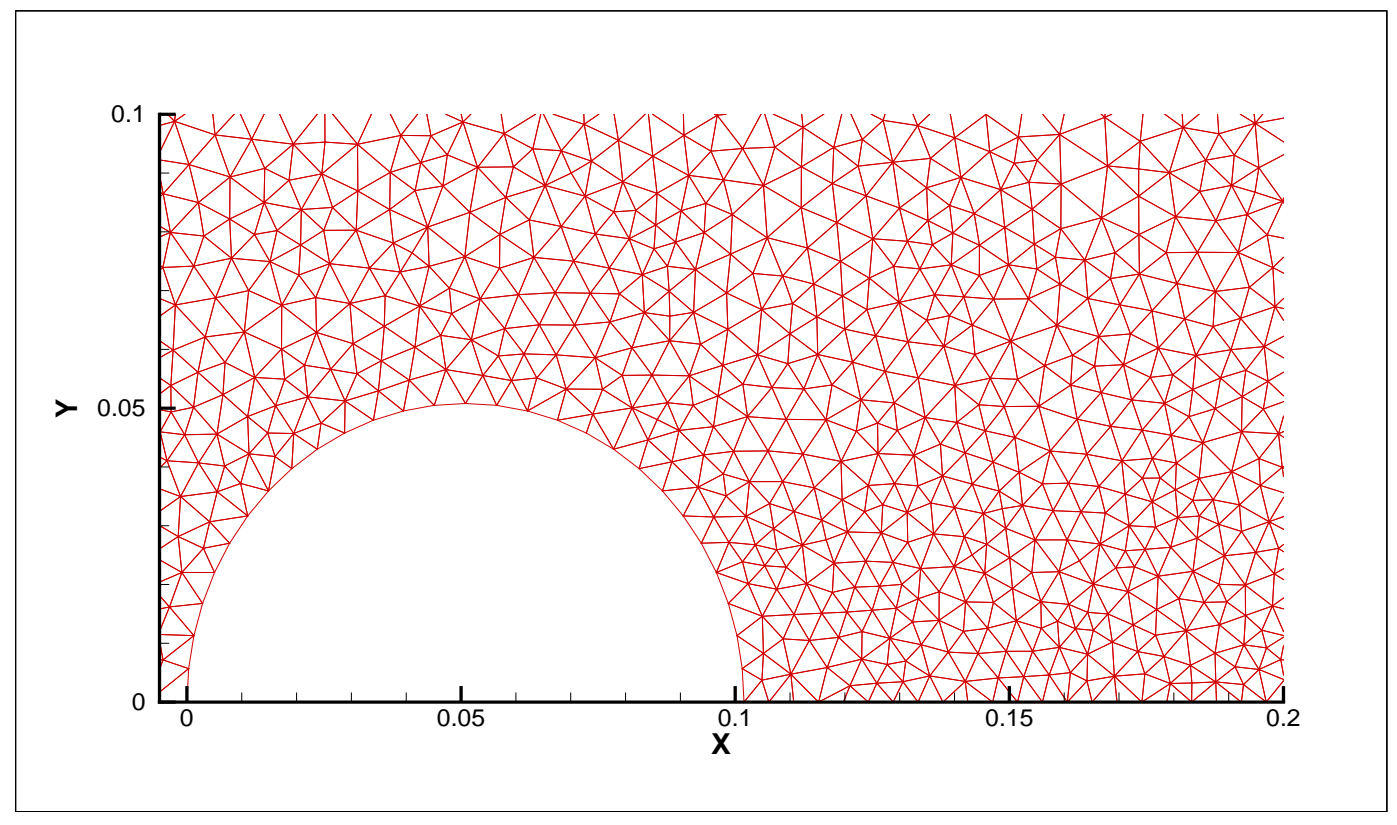

Figura 3.11: zoom da malha $m 4$.

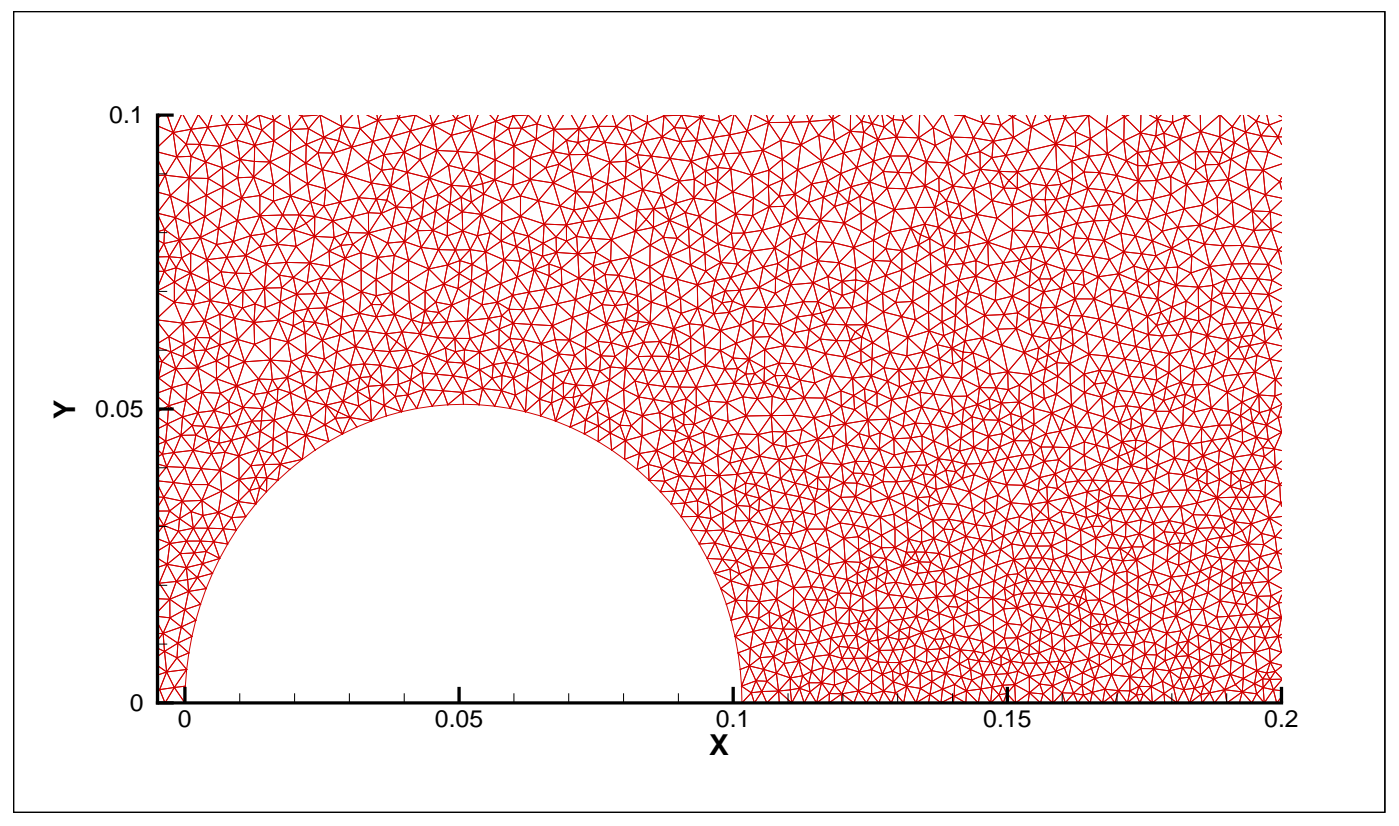

Figura 3.12: zoom da malha $m 5$. 


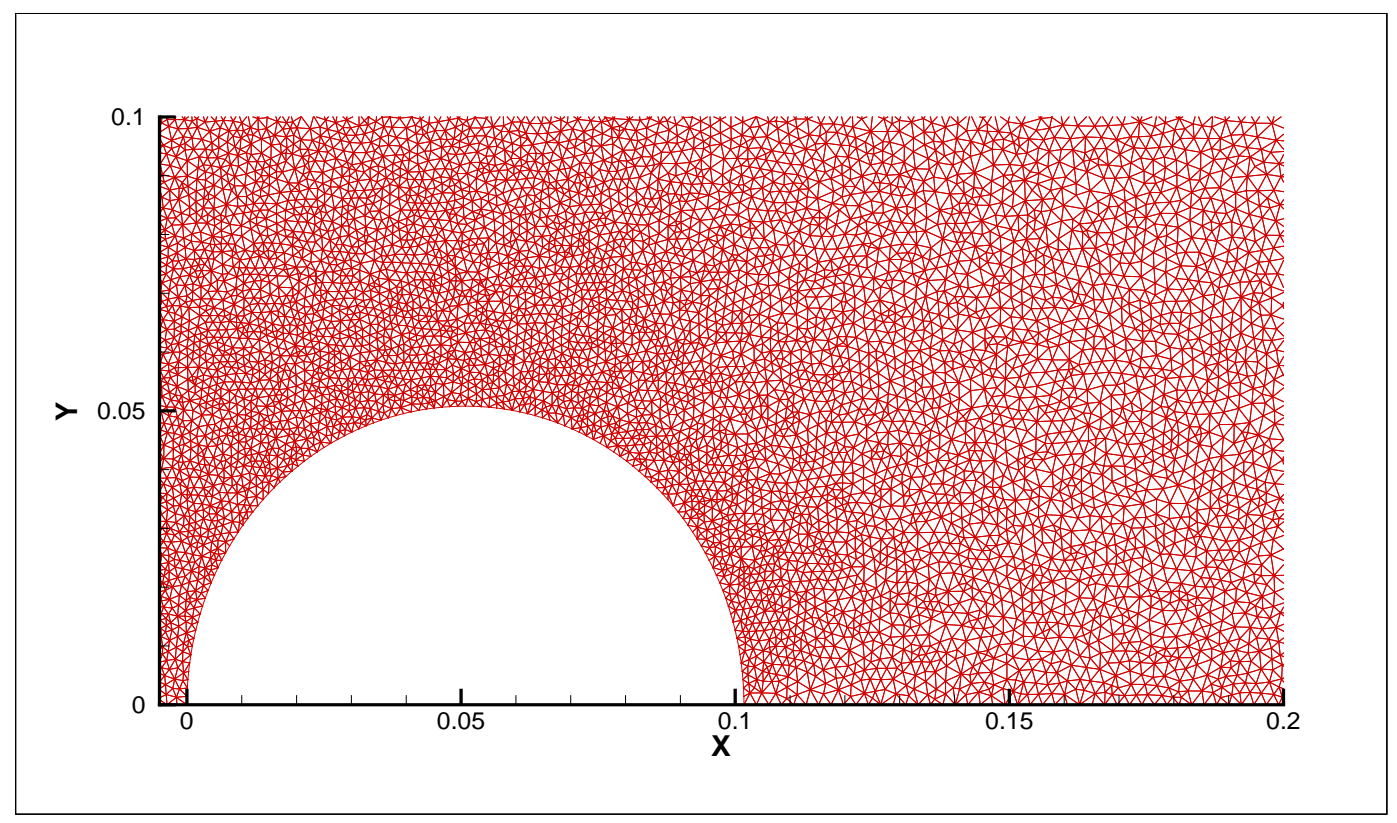

Figura 3.13: zoom da malha $m 6$.

Foi feito um estudo para analisar o efeito da variação do número de Reynolds na região atrás do cilindro. Trabalhou-se com valores de $R e=1.5 \times 10^{5}$ e $R e=6.7 \times 10^{5}$ baseado em [59]. O ponto de descolamento para $R e=1.5 \times 10^{5}$ ocorre no $24^{\mathrm{o}}$ ponto sobre o cilindro no sentido anti-horário, a partir do ponto de coordenadas (D,0), ou seja, aresta com inclinação de $52,23^{\circ}$ em relação ao eixo x , Figura 3.14 (a). Para o teste com $R e=6.7 \times 10^{5}$, o ponto de descolamento ocorre antes, no $20^{\circ}$. ponto (aresta com inclinação de 58,66 em relação ao eixo x), Figura 3.14 (b). A magnitude da velocidade, número de Mach e pressão podem ser vistos nas Figuras 3.15-3.17.

Na Figura 3.15 observa-se a influência da escolha do número de Reynolds atrás do cilindro no valor da magnitude da velocidade e conseqüentemente no Mach, Figura 3.16. 


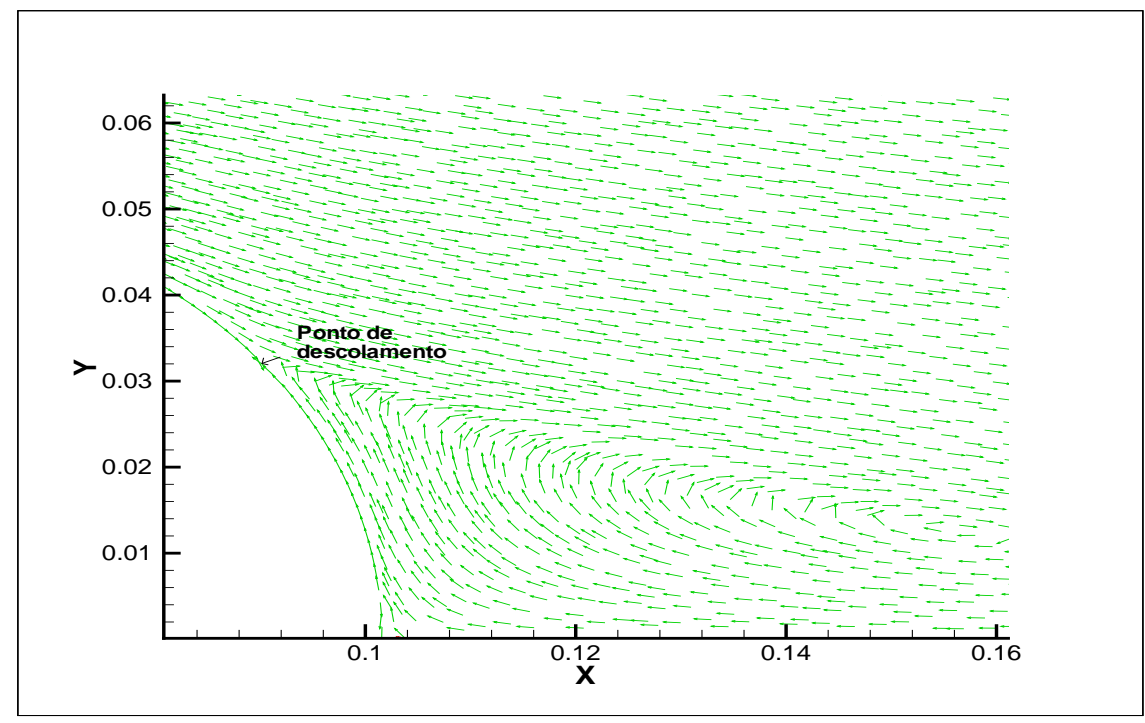

(a)

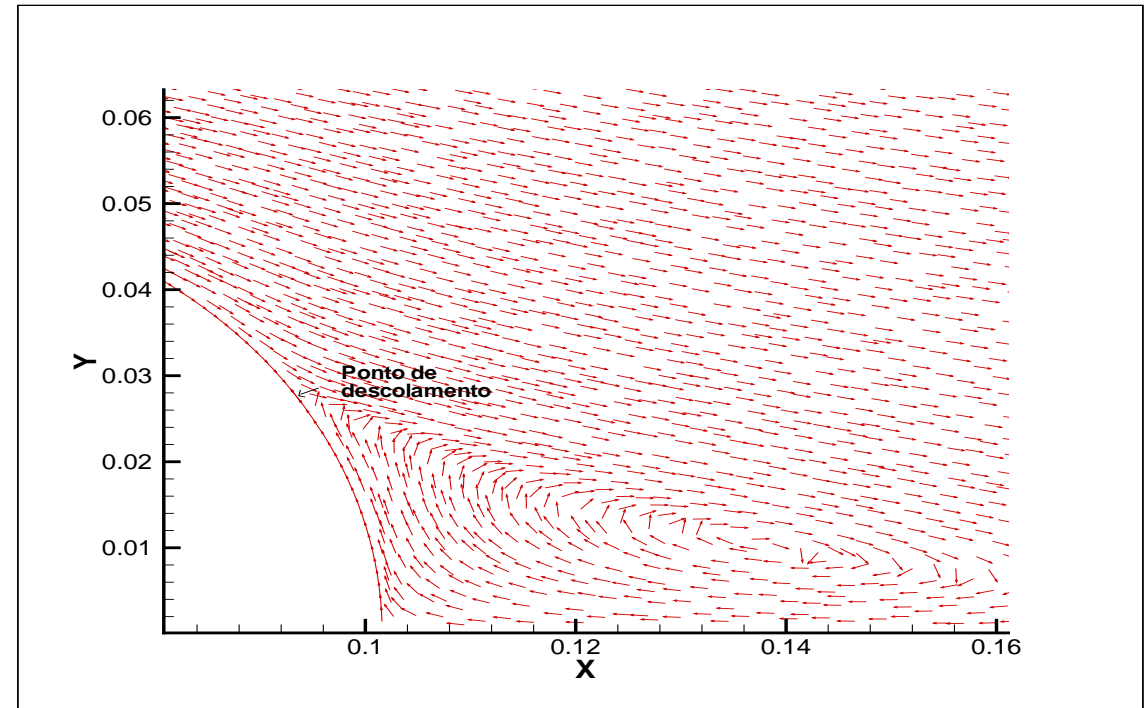

(b)

Figura 3.14: Vetor velocidade na malha m3: (a) $R e=1.5 \times 10^{5}$ e (b) $R e=6.7 \times 10^{5}$. 


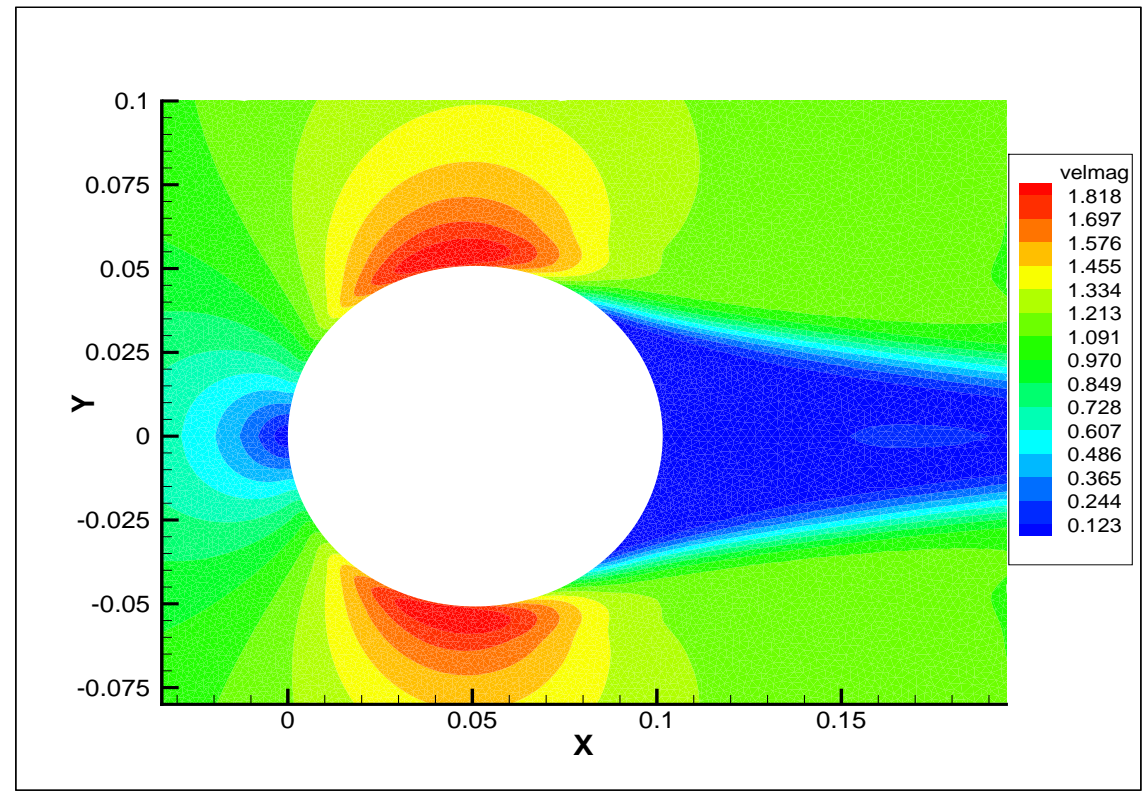

(a)

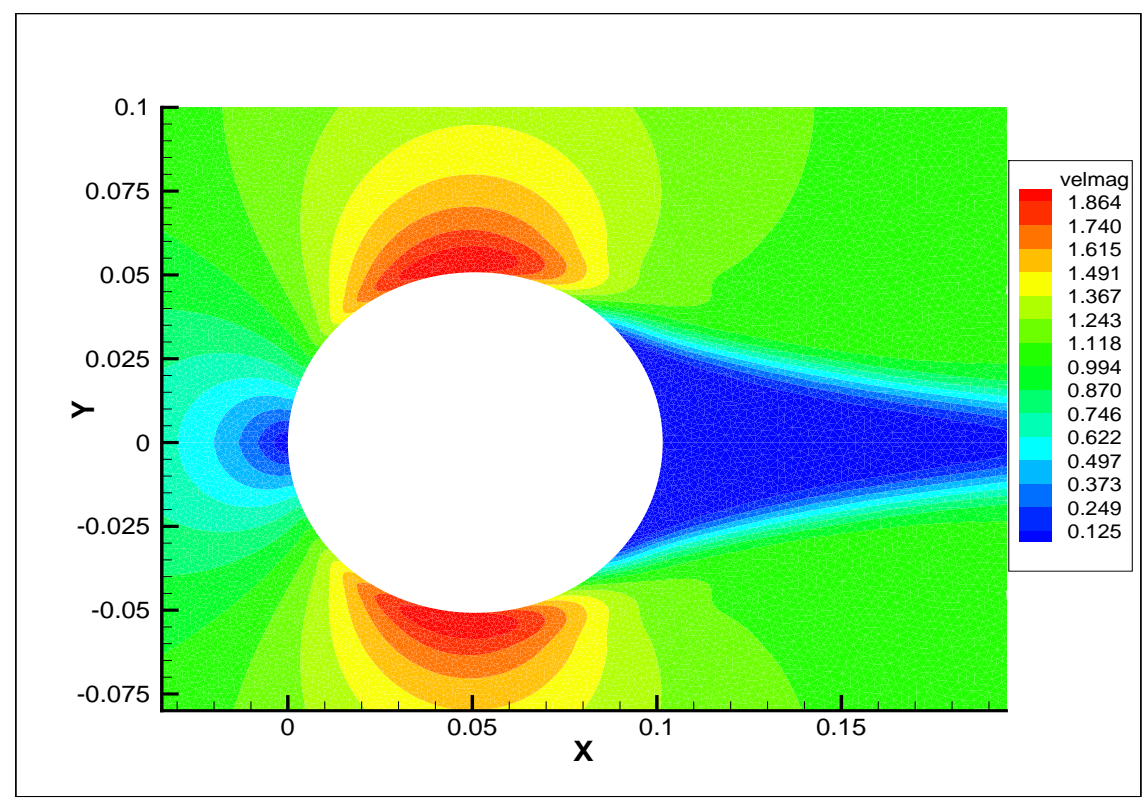

(b)

Figura 3.15: Magnitude da velocidade na malha $m 3$ : (a) $R e=1.5 \times 10^{5}$ e (b) $R e=$ $6.7 \times 10^{5}$. 


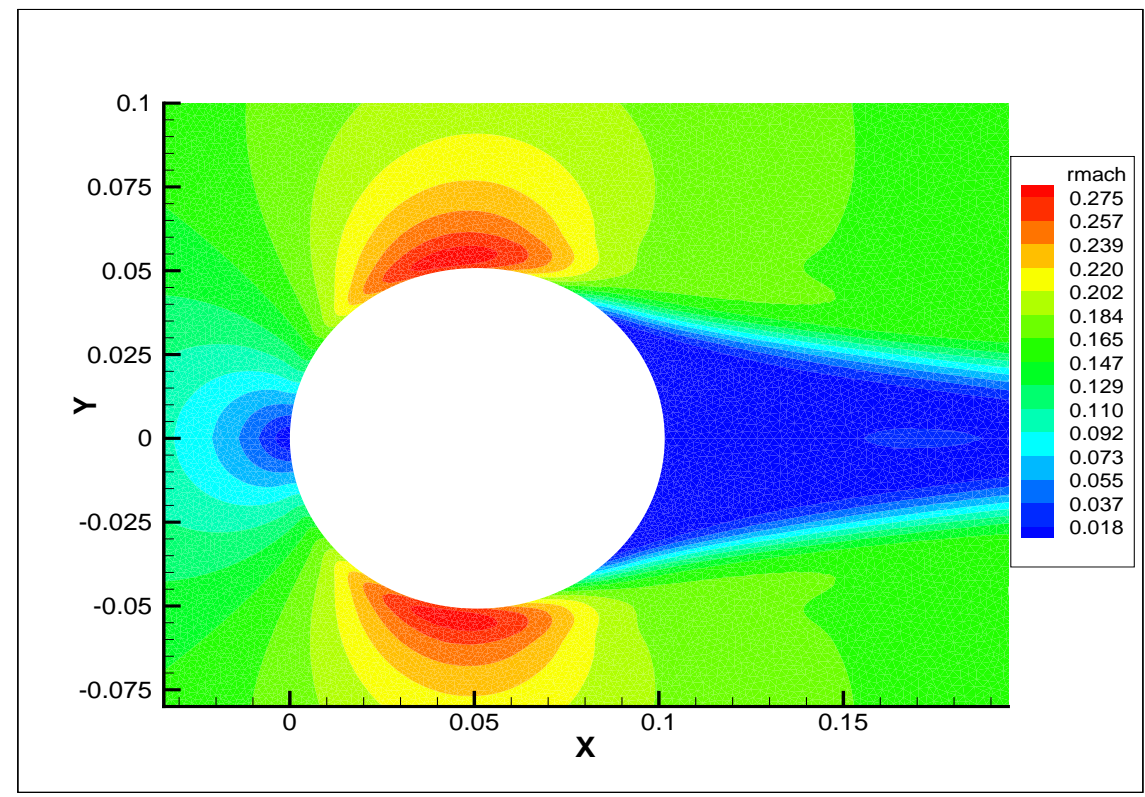

(a)

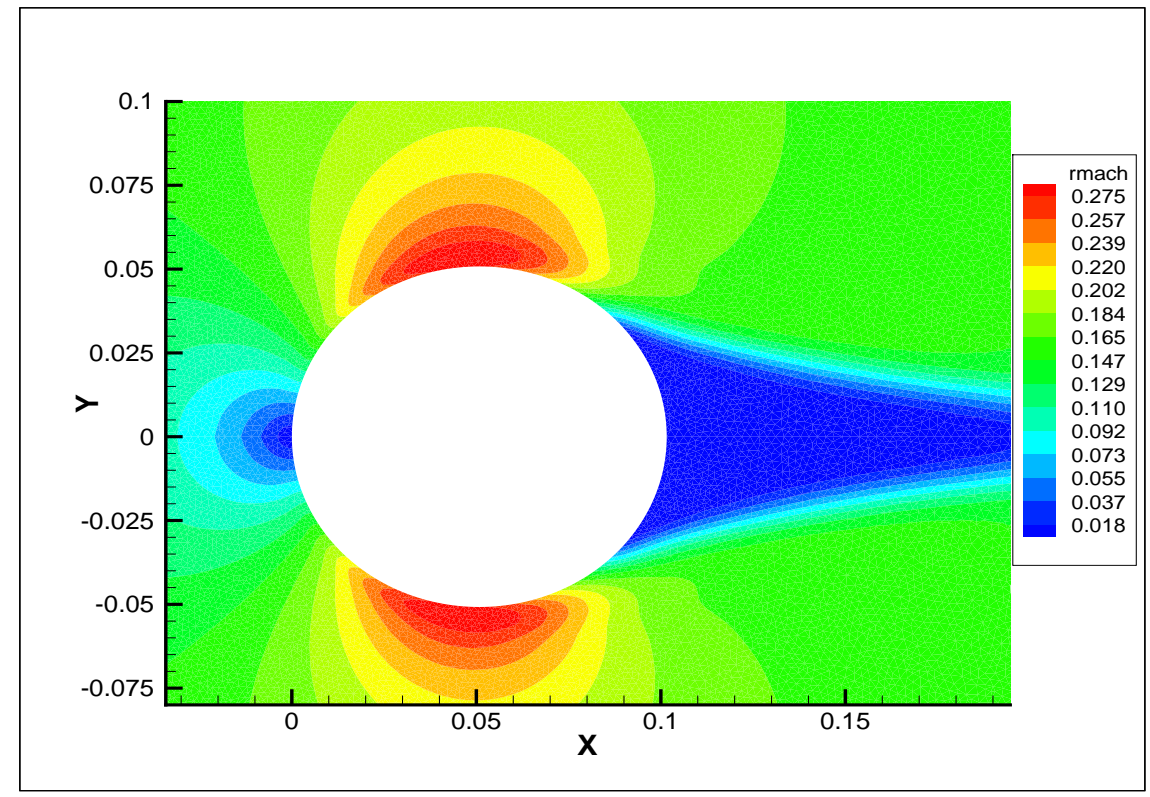

(b)

Figura 3.16: Mach na malha $m 3$ : (a) $R e=1.5 \times 10^{5}$ e (b) $R e=6.7 \times 10^{5}$. 


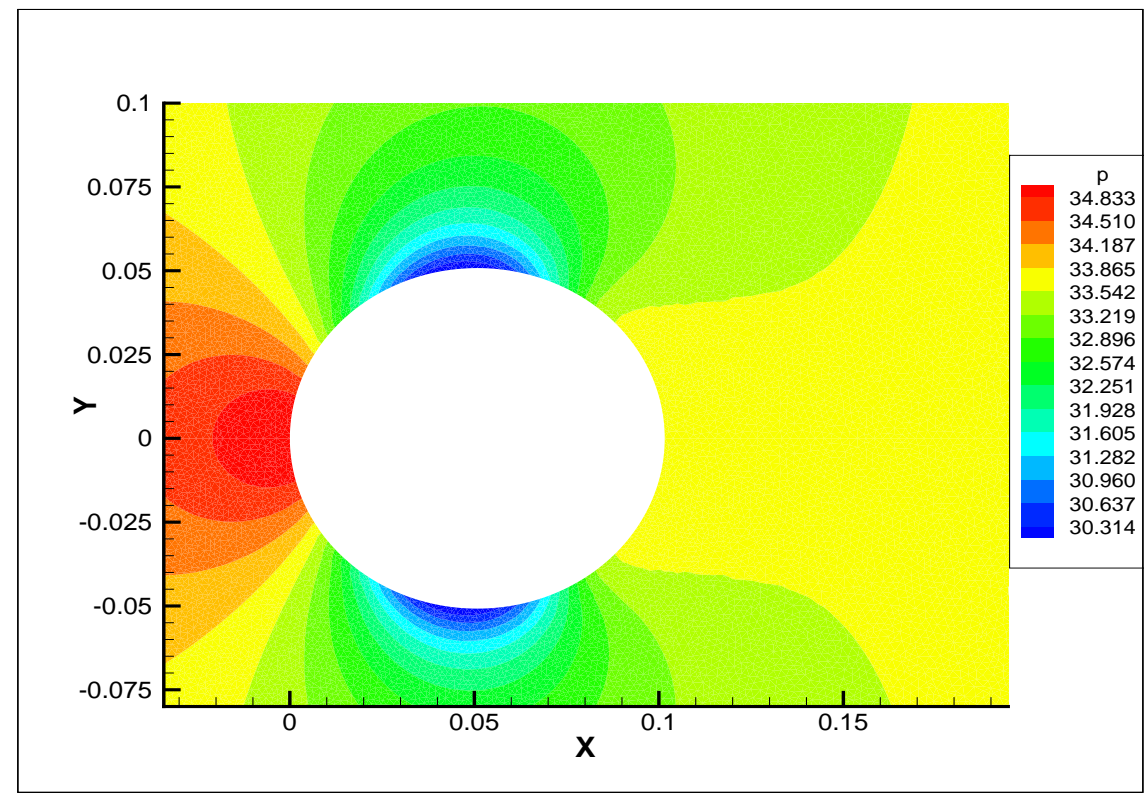

(a)

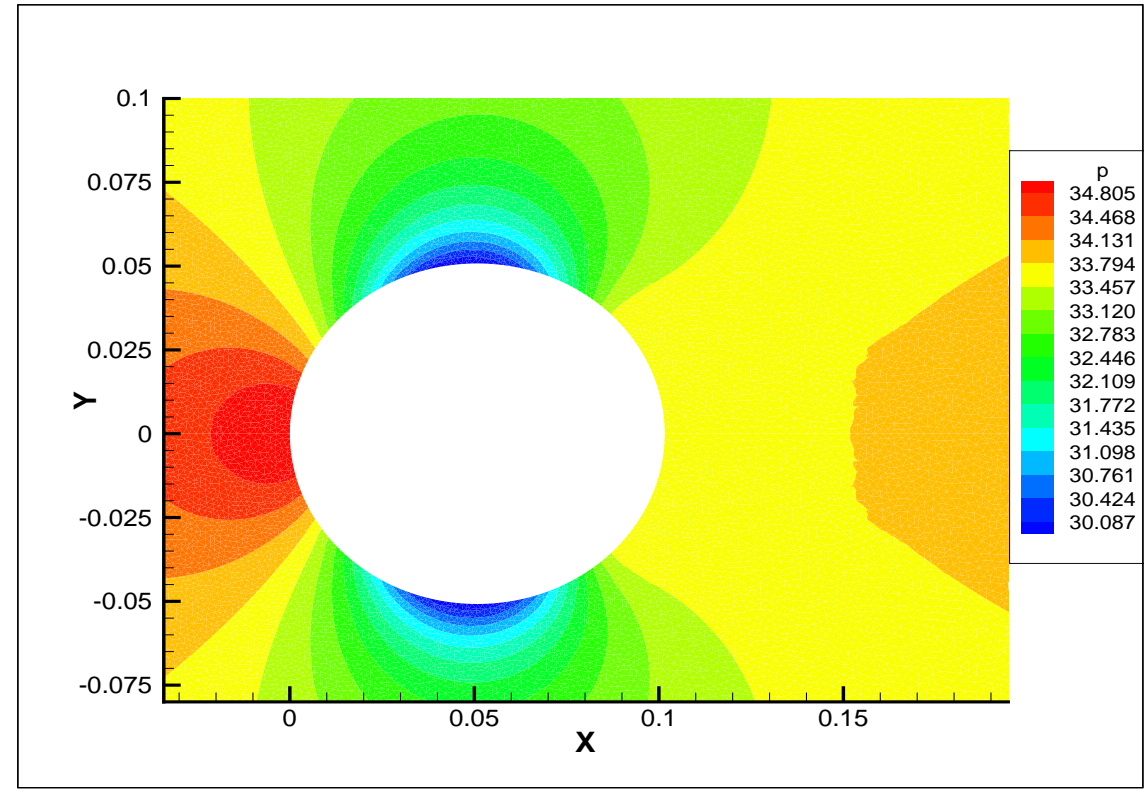

(b)

Figura 3.17: Campo de pressão na malha m3: (a) $R e=1.5 \times 10^{5}$ e (b) $R e=6.7 \times 10^{5}$. 


\section{Reconstrução mínimos quadrados com alta ordem}

A maioria dos pacotes de CFD comerciais possuem aproximação de até segunda ordem de discretização espacial, conseqüentemente requerem uma malha fina quando se deseja alta precisão, o que leva a um alto custo por iteração. Assim, os métodos de reconstrução da solução de alta ordem têm-se apresentado como ferramentas alternativas a serem aplicadas na solução numérica de escoamentos mostrando ser uma opção praticamente mais econômica do que os métodos de $2^{\text {a. }}$ ordem.

Este capítulo inicia-se com uma revisão de algumas técnicas de reconstrução de alta ordem presentes na literatura. Em seguida, é descrito o método de alta ordem com reconstrução mínimos quadrados de Gooch et al. [22, 23, 24, 25, 26, 52, 70], implementado neste trabalho.

\subsection{As técnicas de reconstrução de alta ordem}

Vários esquemas de alta ordem foram desenvolvidos para atender precisões de alta ordem para soluções suaves, com uma baixa ordem de precisão próximo das descontinuidades e nos extremos da solução [52]. Algumas características destas técnicas são apresentadas a seguir $[1,2,35,37,40]$.

O FCT (Flux Corrected Transport) e o TVD (Total Variation Diminishing) são métodos de alta ordem que começaram em malhas estruturadas. Uma soma de limitantes de flu- 
xos anti-difusivos é adicionado para manter a monotonicidade, empregando limitantes não-lineares em toda a região do campo do escoamento, o qual pode ser afetado pela convergência.

O MUSCL (Monotonic Upstream Scheme for Conservation Law) desenvolvido por Van Leer, inicialmente usava malhas estruturadas e mais tarde passou para malhas nãoestruturadas. Conta com uma reconstrução por segmentos lineares usando limitantes não-lineares forçando uma monotonicidade. Possui baixa precisão nas descontinuidades e extremos. O MUSCL está focado em conseguir precisão de alta ordem para soluções suaves, com uma queda da precisão próximo à descontinuidade e extremos na solução.

Mais recentemente, esquemas ENO (Essentially Non-Oscillatory) [1] tem sido desenvolvido para garantir uma precisão mais uniforme para todos os pontos com uma vizinhança suave, usa reconstrução por polinômios para alta ordem mas não tem demonstrado praticidade quanto à procura do estêncil "mais suave" para a reconstrução em cada volume de controle, como pode ser observado na Figura 4.1.

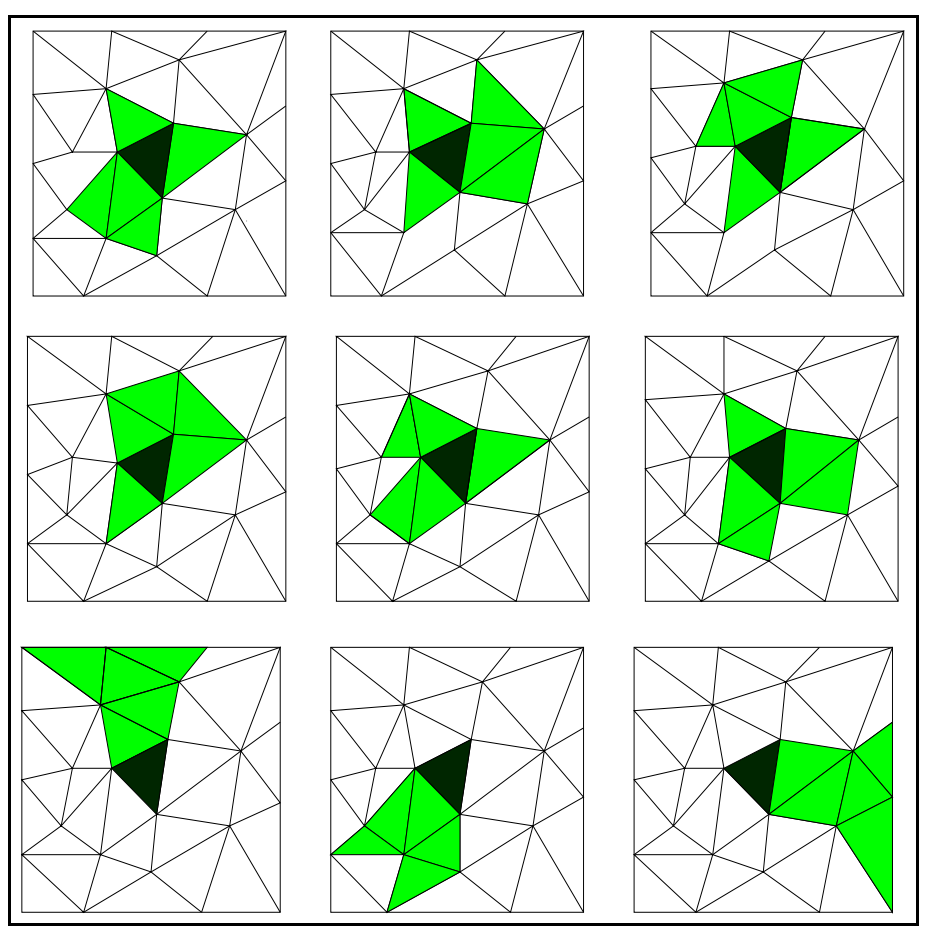

Figura 4.1: Exemplo de possíveis estênceis no esquema ENO.

A busca do estêncil no esquema ENO pode trazer o problema que pequenas mudanças 
na solução no processo iterativo pode causar estênceis switching e promover a convergência para o estado estacionário numérico e não a solução do problema.

Isso tem sido administrado pela nova família de esquemas Weighted ENO (WENO) $[35, ?]$. Esse esquema usa a soma de todos os estênceis possíveis procurando pelo estêncil mais suave possível, usando todos os polinômios possíveis na forma de pesos dependentes dos dados, os pesos são na ordem do erro de truncamento em estênceis não-suaves.

O método a ser descrito a seguir tem uma importante diferença com os esquemas WENO, possui um bom comportamento quando um estêncil suave não existe.

\subsection{O método da reconstrução mínimos quadrados}

Infelizmente, a implementação de esquemas que utiliza a busca de estênceis em malhas não-estruturadas não é fácil pois estes devem ser procurados em todas as direções simultaneamente e o número de prováveis estênceis é muito grande como pôde ser observado na Figura 4.1.

A proposta de Gooch e colaboradores [22, 23, 24, 25, 26, 52, 70] é o desenvolvimento de uma técnica de alta ordem sem a necessidade dessa busca pelo estêncil mais adequado.

A técnica usa estêncil fixo, pesos dependentes dos dados e a solução das equações de conservação podem ser separadas naturalmente em 3 fases:

i) reconstrução da solução;

ii) integração do fluxo;

iii) evolução temporal.

\subsection{Reconstrução da solução}

O método é baseado na reconstrução mínimos quadrados com dependência de dados, ou seja, para uma função $\phi(\vec{x})$ definida no domínio $\Omega$ e o seu valor médio no volume de controle $\bar{\phi}_{i}(\vec{x})$ calcula-se uma expansão $\phi_{i}^{R}\left(\bar{x}-\bar{x}_{i}\right)$ sobre $i$, do volume de controle $V_{i}$ tal que 
- conserva a média;

- tem suporte compacto;

- reconstrói exatamente o polinômio de grau $\leq \mathrm{k}$, ou seja $\phi_{i}^{R}\left(\vec{x}-\vec{x}_{i}\right)-\phi(\vec{x})=O\left(\Delta x^{k+1}\right)$;

- satisfaz as propriedades ENO de Liu, Osher \& Chan.

O método dos volumes finitos usa um ponto de referência local no volume de controle como a origem para a reconstrução polinomial. Esse ponto de referência é o centróide do triângulo, cell-centred, Figura 4.2 (a), ou o vértice comum aos triângulos vizinhos, cell-vertex, Figura 4.2 (b), para os volumes de controle centrado na célula ou no vértice, respectivamente.

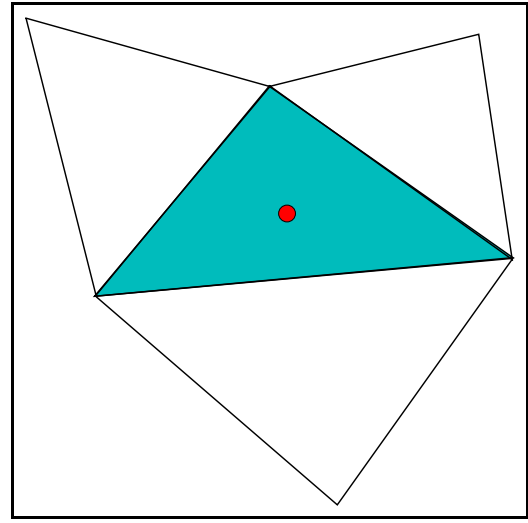

(a)

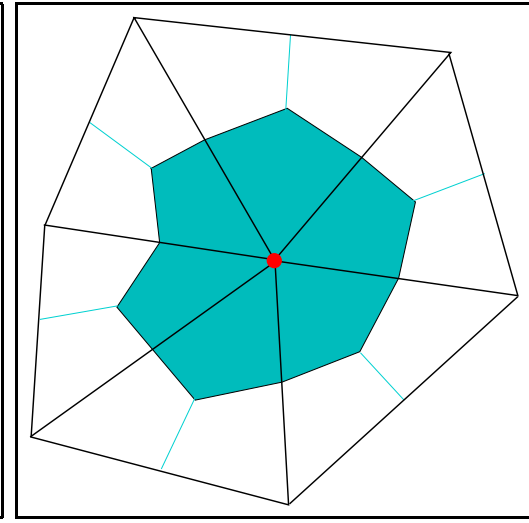

(b)

Figura 4.2: (a) Volume de controle centrado na célula; (b) volume de controle centrado no vértice [70].

Tanto para volume de controle centrado no vértice ou na célula, Figura 4.2 (a) e (b), a idéia é repassar o valor da média do volume de controle da solução, $\bar{\phi}_{i}$, como uma expansão da série de Taylor. O número mínimo de volumes de controle vizinhos no estêncil de reconstrução é igual ao número de termos derivados a serem aproximados.

No pré-processamento, o desenvolvimento de uma estrutura de dados é uma importante etapa para encontrar os vizinhos de segunda, terceira ou quarta ordem de cada vértice, para volumes de controle centrados no vértice, Figura 4.3 (a), ou de cada centróide para volume de controle centrado na célula, Figura 4.3 (b). Além disto, é necessário determinar as conectividades entre os centróides, vértices e faces do volume de controle. 


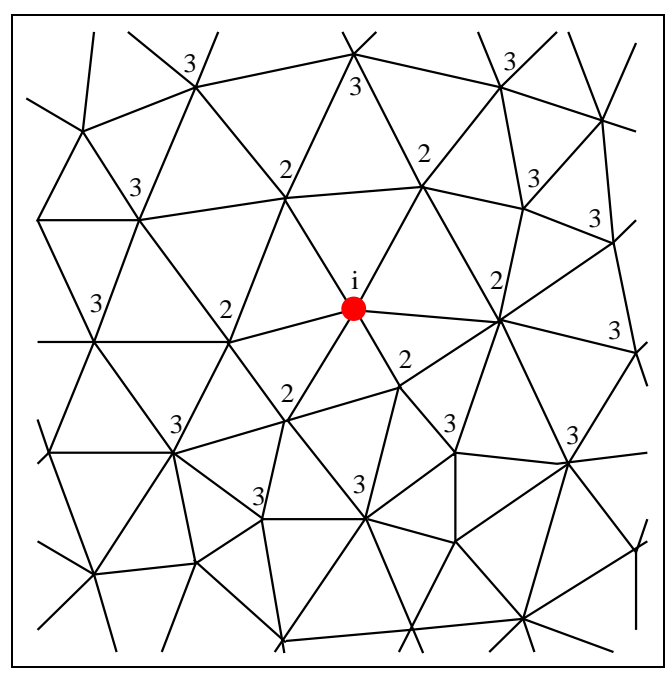

(a)

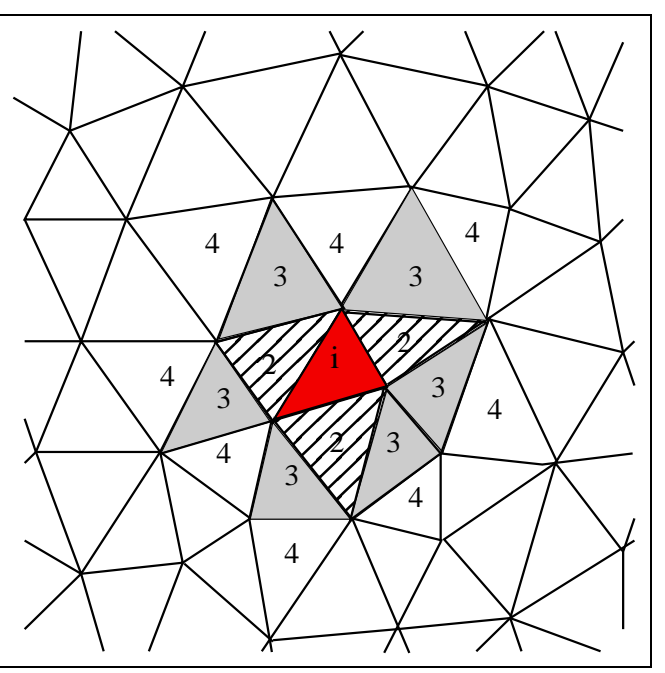

(b)

Figura 4.3: Estêncil de parte da malha para reconstrução de alta ordem: $2^{a}$, $3^{a}$ e $4^{a}$. ordem, respectivamente: (a) do volume de controle centrado no vértice $i$ e (b) do volume de controle centrado na célula $i$, adaptado de [70].

Assim, no desenvolvimento do código uma estrutura de dados foi construída para percorrer as faces do triângulo, adaptada de Lindquist [39]. A Figura 4.4 mostra os elementos utilizados na elaboração desta estrutura.

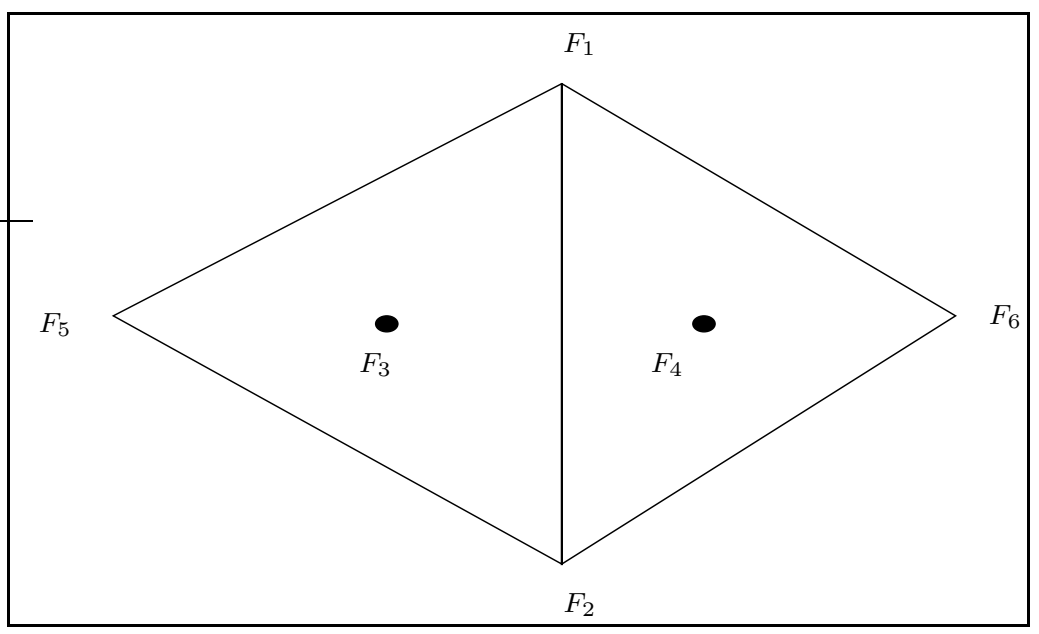

Figura 4.4: Elementos triangulares com uma face em comum $\left(\overline{F_{1} F_{2}}\right)$.

$\mathrm{O}$ vetor que armazena os dados, considerando $\overline{F_{1} F_{2}}=i$, índice da face, é dado por 


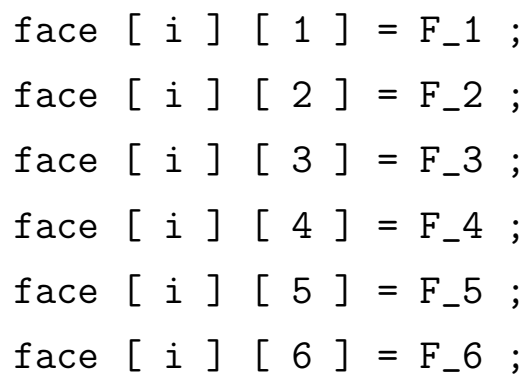

Se um nó ou face pertence ao exterior do domínio computacional seus valores são iguais a zero. Assim, essa estrutura possibilitou, apartir do arquivo obtido pelo gerador de malhas EMC2, determinar os vizinhos de um vértice para o cálculo dos demais elementos necessários na reconstrução de alta ordem, estabelecer quais vértices, triângulos estão na fronteira e quais são os seus vizinhos para obter o polinômio de reconstrução.

Para implementar a condição de contorno é necessário reconhecer quais faces ou células estão no contorno e suas orientações neste contorno. A orientação adotada é CCW (clock counter wise), ou seja, no sentido anti-horário.

Uma vez implementada a estrutura de dados, calcula-se todos os momentos nos volumes de controle que serão utilizados na montagem do problema mínimos quadrados bem como os termos geométricos e as restrições para um vértice do contorno, seção 4.4.

Com isso, o ponto crucial para obter uma solução de alta ordem é a reconstrução de alta ordem apartir dos valores das médias nos volumes de controle. A reconstrução deve conservar o valor médio da função para cada volume de controle e ainda ter boas propriedades de convergência, ser computacionalmente eficiente e ser de fácil implementação em uma malha arbitrária.

Segundo Gooch [23], este esquema de reconstrução tem as melhores propriedades de ambos, do esquema k-Exato Limitado [4, 5, 6, 7, 8, 88] e do esquema ENO.

O desenvolvimento do método será feito para volumes de controle centrados no vértice (cell vertex), cuja vantagem é que a reconstrução encontrada já está no vértice, eliminando a necessidade de uma interpolação para os mesmos para determinar os valores a serem calculados. Além disso, o número de vértices é menor que o número de triângulos.

Assim, dados valores discretos de uma quantidade escalar $\phi_{i}$ para o ponto $i$ da malha, um polinômio de reconstrução $\phi_{i}^{R}$ para um ponto arbitrário $(x, y)$, em duas dimensões, 
próximo ao ponto $i$ é definido pela série de Taylor (2D):

$$
\begin{aligned}
\phi_{i}^{R}(x, y) & =\phi_{i}+\left.\frac{\partial \phi}{\partial x}\right|_{i}\left(x-x_{i}\right)+\left.\frac{\partial \phi}{\partial y}\right|_{i}\left(y-y_{i}\right)+\left.\frac{\partial^{2} \phi}{\partial x^{2}}\right|_{i} \frac{\left(x-x_{i}\right)^{2}}{2}+\left.\frac{\partial^{2} \phi}{\partial x \partial y}\right|_{i}\left(x-x_{i}\right)\left(y-y_{i}\right)+ \\
& +\left.\frac{\partial^{2} \phi}{\partial y^{2}}\right|_{i} \frac{\left(y-y_{i}\right)^{2}}{2}+\cdots
\end{aligned}
$$

onde:

$\phi$ é o valor da solução reconstruída;

$\frac{\partial^{k+l} \phi_{i}}{\partial x^{l} \partial y^{k}}$ são suas derivadas para o ponto de referência $\left(x_{i}, y_{i}\right)$ do volume de controle $i$.

A escolha dos coeficientes da expansão é feita de forma a conservar o valor médio no volume de controle e minimizar o erro na representação de uma solução suave, como mostra (4.2).

\subsubsection{Conservação da média}

O valor médio para um único volume de controle $V_{j}$ da função reconstruída $\phi_{i}^{R}$ é dado por

$$
\frac{1}{A_{i}} \int_{V_{i}} \phi_{i}^{R} d A=\bar{\phi}_{i}
$$

Expandindo o lado esquerdo usando a série de Taylor, tem-se que

$$
\begin{gathered}
\frac{1}{A_{i}} \int_{V_{i}} \phi_{i}^{R}\left(\vec{x}-\overrightarrow{x_{i}}\right) d A=\phi_{i}+\left.\frac{\partial \phi}{\partial x}\right|_{i} \frac{1}{A_{j}} \int_{V_{j}}\left(x-x_{i}\right) d A+\left.\frac{\partial \phi}{\partial y}\right|_{i} \frac{1}{A_{j}} \int_{V_{j}}\left(y-y_{i}\right) d A+ \\
\left.\frac{\partial^{2} \phi}{\partial x^{2}}\right|_{i} \frac{1}{2 A_{j}} \int_{V_{j}}\left(x-x_{i}\right)^{2} d A+\left.\frac{\partial^{2} \phi}{\partial x \partial y}\right|_{i} \frac{1}{A_{j}} \int_{V_{j}}\left(x-x_{i}\right)\left(y-y_{i}\right) d A+ \\
\left.\frac{\partial^{2} \phi}{\partial y^{2}}\right|_{i} \frac{1}{2 A_{j}} \int_{V_{j}}\left(y-y_{i}\right)^{2} d A+\cdots
\end{gathered}
$$

Para evitar o cálculo do momento em cada volume de controle em $\left\{V_{j}\right\}_{i}$ sobre $V_{i}$, substitui-se $\left(x-x_{i}\right)$ por $\left(x-x_{j}\right)+\left(x_{j}-x_{i}\right)$ e $\left(y-y_{i}\right)$ por $\left(y-y_{j}\right)+\left(y_{j}-y_{i}\right)$, respectivamente. Os volumes monomiais (momentos) podem ser expressos usando o Teorema de Green para convertê-los em uma integral de contorno e eles podem ser integrados exatamente usando quadratura gaussiana com pontos e pesos nos triângulos como mostrado na Tabela B.1, Apêndice B. 
Expandindo e integrando (4.3):

$$
\begin{aligned}
& \frac{1}{A_{j}} \int_{V_{j}} \phi_{i}^{R}\left(\vec{x}-\vec{x}_{i}\right) d A=\phi_{i}+\left.\frac{\partial \phi}{\partial x}\right|_{i} \int_{V_{j}}\left(\bar{x}_{j}+\left(x_{j}-x_{i}\right)\right)+\left.\frac{\partial \phi}{\partial y}\right|_{i} \int_{V_{j}}\left(\bar{y}_{j}+\left(y_{j}-y_{i}\right)\right)+ \\
& +\left.\frac{\partial^{2} \phi}{\partial x^{2}}\right|_{i} \int_{V_{j}} \frac{\bar{x}_{j}^{2}+2 \bar{x}_{j}\left(x_{j}-x_{i}\right)+\left(x_{j}-x_{i}\right)^{2}}{2}+\left.\frac{\partial^{2} \phi}{\partial x \partial y}\right|_{i} \int_{V_{j}}\left[\overline{x y} \bar{y}_{j}^{2}+\bar{x}_{j}\left(y_{j}-y_{i}\right)+\right. \\
& \left.+\left(x_{j}-x_{i}\right) \bar{y}_{j}+\left(x_{j}-x_{i}\right)\left(y_{j}-y_{i}\right)\right]+\left.\frac{\partial^{2} \phi}{\partial y^{2}}\right|_{i} \int_{V_{j}} \frac{\bar{y}_{j}^{2}+2 \bar{y}_{j}\left(y_{j}-y_{i}\right)+\left(y_{j}-y_{i}\right)^{2}}{2}+\cdots
\end{aligned}
$$

O cálculo da restrição $\overline{x^{n} y^{n}}$ é dado por:

$$
{\overline{x^{n} y^{n}}}_{i}=\frac{1}{A_{i}} \int_{V_{i}}\left(x-x_{i}\right)^{n}\left(y-y_{i}\right)^{m} d A,
$$

que pode ser convertido em

$$
\begin{gathered}
{\overline{x^{m} y^{n}}}_{i}=\frac{1}{(n+1) A_{i}} \int_{\partial V_{i}}\left(x-x_{i}\right)^{(n+1)}\left(y-y_{i}\right)^{m} \hat{n}_{x} d s \\
\overline{x^{m} y^{n}}{ }_{i}=\frac{1}{(n+1) A_{i}} \sum_{j=1}^{j \max } \sum_{k=1}^{N g}\left[\left(x_{g}-x_{i}\right)^{n+1}\left(y_{g}-y_{i}\right)^{m} \hat{n}_{x g} \cdot w_{g}\right]_{k}
\end{gathered}
$$

onde, $N_{g}$ é número de pontos de Gauss para que a integral seja exata.

Nestes termos,

$$
\begin{aligned}
\frac{1}{A_{j}} \int_{V_{j}} \phi_{i}^{R}\left(\vec{x}-\vec{x}_{i}\right) d A= & \left.\phi\right|_{i}+\left.\frac{\partial \phi}{\partial x}\right|_{i} \hat{x}_{i j}+\left.\frac{\partial \phi}{\partial y}\right|_{i} \hat{y}_{i j}+\left.\frac{\partial^{2} \phi}{\partial x^{2}}\right|_{i} \frac{x^{2}}{2}+ \\
& \left.\frac{\partial^{2} \phi}{\partial x \partial y}\right|_{i} \widehat{x y}_{i j}+\left.\frac{\partial^{2} \phi}{\partial y^{2}}\right|_{i} \frac{\widehat{y}_{i j}^{2}}{2}+\cdots
\end{aligned}
$$

Com os termos geométricos na forma:

$$
\begin{aligned}
\widehat{x^{n} y_{i j}^{m}} & \equiv \frac{1}{A_{j}} \int_{V_{i}}\left(\left(x-x_{j}\right)+\left(x_{j}-x_{i}\right)\right)^{n} \cdot\left(\left(y-y_{j}\right)+\left(y_{j}-y_{i}\right)\right)^{m} d A \\
& =\sum_{l=0}^{m} \sum_{k=0}^{n}\left(\begin{array}{c}
m \\
l
\end{array}\right)\left(\begin{array}{c}
n \\
k
\end{array}\right)\left(x_{j}-x_{i}\right)^{k} \cdot\left(y_{j}-y_{i}\right)^{l} \cdot \overline{x^{n-k} y_{j}^{m-l}} \\
& =\sum_{l=0}^{m}\left\{\frac{m !}{l !(m-l) !}\left(y_{j}-y_{i}\right)^{l} \sum_{k=0}^{n}\left[\frac{n !}{k !(n-k) !}\left(x_{j}-x_{i}\right)^{k} \overline{x^{n-k} y^{m-l}}\right]\right\}
\end{aligned}
$$


onde

$$
\overline{x^{n-k} y_{j}^{m-l}}=\frac{1}{(n-k+1) A_{j}} \int_{\partial V_{j}}(x-x j)^{n-k}\left(y-y_{l}\right)^{m-l} d y .
$$

A equação (4.4) avalia o valor médio da reconstrução de $\phi_{i}^{R}\left(\vec{x}-\vec{x}_{i}\right)$ para um volume de controle $j$. Com isto em mãos, pode-se escrever o problema mínimos quadrados para as derivadas,

$$
\left(\begin{array}{ccccccc}
1 & \bar{x}_{i} & \bar{y}_{i} & \bar{x}^{2}{ }_{i} & \overline{x y}_{i} & \bar{y}^{2}{ }_{i} & \cdots \\
\hline w_{i 1} & w_{i 1} \hat{x}_{i 1} & w_{i 1} \hat{y}_{i 1} & w_{i 1} \hat{x}_{i 1}^{2} & w_{i 1} \widehat{x y}_{i 1} & w_{i 1} \hat{y}_{i 1}^{2} & \cdots \\
w_{i 2} & w_{i 2} \hat{x}_{i 2} & w_{i 2} \hat{y}_{i 2} & w_{i 1} \hat{x}_{i 2}^{2} & w_{i 2} \widehat{x y}_{i 2} & w_{i 2} \hat{y}_{i 2}^{2} & \cdots \\
w_{i 3} & w_{i 3} \hat{x}_{i 3} & w_{i 3} \hat{y}_{i 3} & w_{i 1} \hat{x}_{i 3}^{2} & w_{i 3} \widehat{x y}_{i 3} & w_{i 3} \hat{y}_{i 3}^{2} & \cdots \\
\vdots & \vdots & \vdots & \vdots & \vdots & \vdots & \ddots \\
w_{i N} & w_{i 4} \hat{x}_{i N} & w_{i N} \hat{y}_{i N} & w_{i 1} \hat{x}_{i N}^{2} & w_{i N} \widehat{x y}_{i N} & w_{i N} \hat{y}_{i N}^{2} & \cdots
\end{array}\right)\left(\begin{array}{c}
\phi \\
\frac{\partial \phi}{\partial x} \\
\frac{\partial \phi}{\partial y} \\
\frac{\partial^{2} \phi}{\partial x^{2}} \\
\frac{\partial^{2} \phi}{\partial x \partial y} \\
\frac{\partial^{2} \phi}{\partial y^{2}} \\
\vdots \\
\frac{\partial^{n} \phi}{\partial x^{n}}
\end{array}\right)_{i}=\left(\begin{array}{c}
\bar{\phi}_{i} \\
\hline w_{i 1} \bar{\phi}_{1} \\
w_{i 2} \bar{\phi}_{2} \\
w_{i 3} \bar{\phi}_{3} \\
\vdots \\
w_{i N} \bar{\phi}_{N}
\end{array}\right)
$$

onde,

$w_{i j}=\frac{1}{\left|\vec{x}_{j}-\vec{x}_{i}\right|^{2}}$;

$\mathrm{N}$ : número de volumes de controle próximos no estêncil.

Os pesos $w_{i j}$ são usados para especificar a importância relativa de uma boa predição para os vários volumes de controle no estêncil e são baseados na distância entre os pontos de referência dos volumes de controle. Os pesos foram introduzidos em malhas nãoestruturadas por Barth $[4,5,6,7,8]$ e tem a vantagem de reduzir a influência dos dados mais distantes do volume de controle de reconstrução. Gooch [27], em estudos recentes, mostra que o valor do expoente no deminador do termo $w_{i j}$ pode ser diferente de 2 .

O número de volumes de controles vizinhos usados no problema mínimos quadrados depende da ordem de precisão desejada, como mostra a Figura 4.5.

Para esquemas de segunda ordem de precisão são necessárias as informações nos vértices vizinhos ao vértice i. Para isto, é preciso reconstruir a função $\phi$ resolvendo o sistema linear de ordem $N \times 3$, N é o número de vizinhos para determinar $\phi, \phi_{x}$ e $\phi_{y}$. 


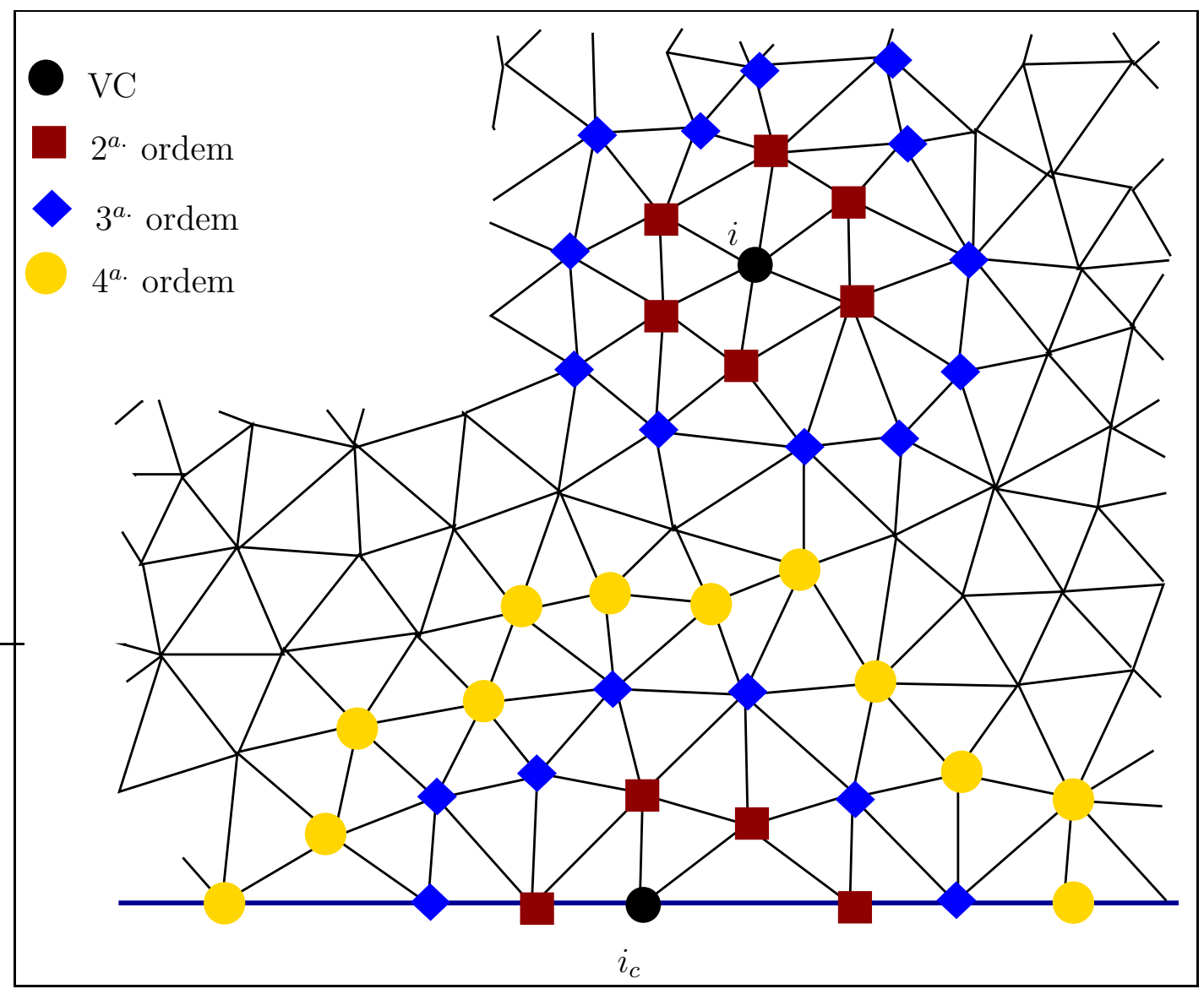

Figura 4.5: Vizinhos da reconstrução de alta ordem com volume de controle centrado no vértice: $i_{c}$ é um vértice do contorno e $i$ é um vértice do interior, adaptado de [70]. 


\subsection{Condição de contorno forçada}

A reconstrução de alta ordem para o volume de controle do contorno deve ser consistente com a condição de contorno para que o esquema apresentado seja totalmente de alta ordem.

Nos volumes de controle do interior a eliminação da analiticidade é direta, seção 4.3, mas para os volumes de controle do contorno, onde a solução deve ser restrita para as condições de contorno forçada então a eliminação da restrição média pode ser mais difícil.

Van Altena [70] apresenta duas estratégias para gerar a restrição no contorno: a restrição de Taylor e a restrição de Gauss, mostrando que a primeira apresenta um certo número de desvantagens em relação à segunda, que é uma forma mais simples de gerar a restrição desejada.

Enquanto o método da restrição de Taylor força a condição de contorno ao longo de todas as faces do volume de controle, o método da restrição de Gauss é parcimonioso, no sentido que as condições de contorno são forçadas somente para os pontos onde os dados do contorno são usados: os pontos de integração de Gauss ao longo do contorno, fornecendo uma restrição do coeficiente do polinômio de reconstrução por ponto de Gauss e pode ser aplicado em qualquer tipo de face, segmento reto ou curvo, onde os pontos de Gauss e as respectivas normais são conhecidos.

Esta restrição é facilmente acrescentada à matriz mínimos quadrados obtendo o problema em um problema mínimos quadrados restrito. A restrição pode ser forçada para as condições de contorno do tipo Dirichlet, Neumann ou Robin, para cada volume de controle adjacente ao contorno.

De acordo com a ordem de precisão do polinômio de reconstrução, deve-se ter o número de pontos de Gauss na face do contorno. A Tabela 4.1 mostra o número de pontos e o número de termos desconhecidos (incógnitas) a serem determinadas quando se resolve o sistema linear. 


\begin{tabular}{|c|c|c|}
\hline $\begin{array}{c}\text { Ordem de } \\
\text { precisão }\end{array}$ & $\begin{array}{c}\text { Número de restrições } \\
\text { no contorno }\end{array}$ & $\begin{array}{c}\text { Número de termos } \\
\text { desconhecidos }\end{array}$ \\
\hline \hline 2 & 3 & 3 \\
\hline 3 & 3 & 6 \\
\hline 4 & 5 & 10 \\
\hline
\end{tabular}

Tabela 4.1: Número de pontos de Gauss por ordem de precisão da reconstrução de $\phi$.

O número de vértice destacado em itálico indica que há o mesmo número de restrições, ou uma a menos que o número de termos desconhecidos, fazendo a reconstrução ser menos confiável, pois há somente um grau de liberdade para o algoritmo mínimos quadrados calcular os valores desconhecidos corretamente. Para a segunda ordem, isto não é um problema pois a matriz mínimos quadrados de segunda ordem nunca se degenerará com duas restrições e uma intregral constante.

\subsubsection{Contorno restrito para a condição de contorno de Dirichlet}

Supondo que ao longo do contorno $\partial \Omega_{1}$, Figura 4.6, a solução deve satisfazer a condição de contorno de Dirichlet $\phi(\vec{x})=f_{1}(\vec{x})$.

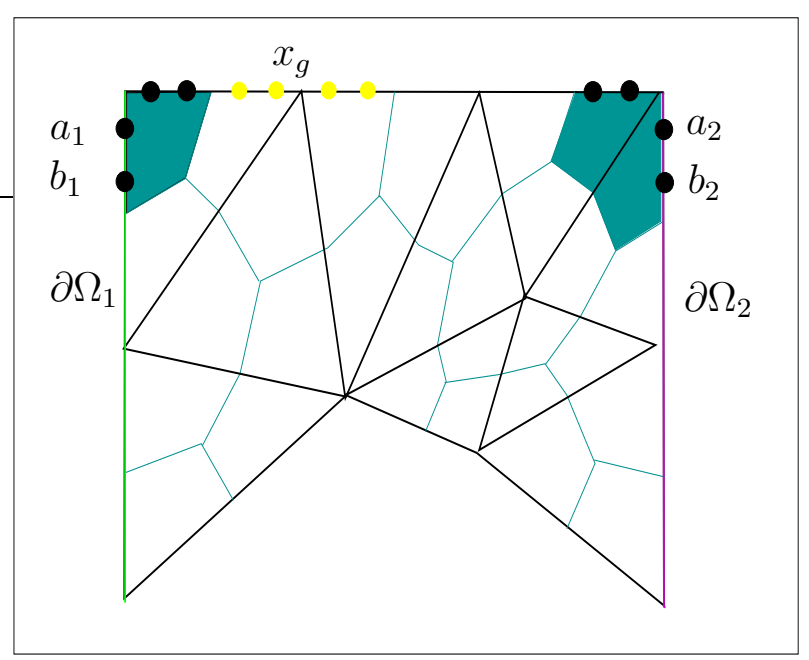

Figura 4.6: Localização dos pontos de Gauss para volume de controle adjacente ao contorno, adaptado de [70]. 
Esta condição é forçada impondo que o valor obtido pelo polinômio de reconstrução da solução no volume de controle deve ser igual ao valor dado pela condição de contorno para cada ponto de integração de Gauss $\left(\vec{x}_{g}\right)$ da face do contorno, ou seja,

$$
\begin{aligned}
f_{1}\left(\vec{x}_{g}\right)=\phi_{1}^{R}\left(\vec{x}_{g}\right) & =\left.\phi\right|_{i}+\left.\frac{\partial \phi}{\partial x}\right|_{i}\left(x_{g}-x_{i}\right)+\left.\frac{\partial \phi}{\partial y}\right|_{i}\left(y_{g}-y_{i}\right)+\left.\frac{\partial^{2} \phi}{\partial x^{2}}\right|_{i} \frac{\left(x_{g}-x_{i}\right)^{2}}{2}+ \\
& +\left.\frac{\partial^{2} \phi}{\partial x \partial y}\right|_{i}\left(x_{g}-x_{i}\right)\left(y_{g}-y_{i}\right)+\left.\frac{\partial^{2} \phi}{\partial y^{2}}\right|_{i} \frac{\left(y_{g}-y_{i}\right)^{2}}{2}+\cdots
\end{aligned}
$$

onde $\left.\phi\right|_{i}$ é o valor da solução reconstruída para o ponto do volume de controle $i$ e assim por diante.

Para cada volume de controle adjacente ao contorno, uma restrição é adicionada ao problema mínimos quadrados como mostra a equação (4.7), para os pontos de Gauss $a_{1}$ e $b_{1}$ da Figura 4.6.

$$
\left(\begin{array}{ccccccc}
1 & \bar{x}_{i} & \bar{y}_{i} & \bar{x}^{2} & \overline{x y}_{i} & \bar{y}^{2}{ }_{i} & \cdots \\
1 & \Delta_{x_{a 1}} & \Delta_{y_{a 1}} & \Delta_{x_{a 1}}^{2} & \Delta_{x_{a 1}} \Delta_{y_{a 1}} & \Delta_{y_{a 1}}^{2} & \cdots \\
1 & \Delta_{x_{b 1}} & \Delta_{y_{b 1}} & \Delta_{x_{b 1}}^{2} & \Delta_{x_{b 1}} \Delta_{y_{b 1}} & \Delta_{y_{b 1}}^{2} & \cdots \\
\hline w_{i 1} & w_{i 1} \hat{x}_{i 1} & w_{i 1} \hat{y}_{i 1} & w_{i 1} \hat{x}_{i 1}^{2} & w_{i 1} \widehat{x y}_{i 1} & w_{i 1} \hat{y}_{i 1}^{2} & \cdots \\
w_{i 2} & w_{i 2} \hat{x}_{i 2} & w_{i 2} \hat{y}_{i 2} & w_{i 2} \hat{x}_{i 2}^{2} & w_{i 2} \widehat{x y}_{i 2} & w_{i 2} \hat{y}_{i 2}^{2} & \cdots \\
w_{i 3} & w_{i 3} \hat{x}_{i 3} & w_{i 3} \hat{y}_{i 3} & w_{i 3} \hat{x}_{i 3}^{2} & w_{i 3} \widehat{x y}_{i 3} & w_{i 3} \hat{y}_{i 3}^{2} & \cdots \\
\vdots & \vdots & \vdots & \vdots & \vdots & \vdots & \ddots \\
w_{i N} & w_{i 4} \hat{x}_{i N} & w_{i N} \hat{y}_{i N} & w_{i N} \hat{x}_{i N}^{2} & w_{i N} \widehat{x y}_{i N} & w_{i N} \hat{y}_{i N}^{2} & \cdots
\end{array}\right)\left(\begin{array}{c}
\phi \\
\frac{\partial \phi}{\partial x} \\
\frac{\partial \phi}{\partial y} \\
\frac{\partial^{2} \phi}{\partial x^{2}} \\
\frac{\partial^{2} \phi}{\partial x \partial y} \\
\frac{\partial^{2} \phi}{\partial y^{2}} \\
\vdots \\
\frac{\partial^{n} \phi}{\partial x^{n}}
\end{array}\right)_{i}=\left(\begin{array}{c}
\bar{\phi}_{i} \\
f_{1}\left(\vec{x}_{a_{1}}\right) \\
f_{1}\left(\vec{x}_{b_{1}}\right) \\
\hline w_{i 1} \bar{\phi}_{1} \\
w_{i 2} \bar{\phi}_{2} \\
w_{i 3} \bar{\phi}_{3} \\
\vdots \\
w_{i N} \bar{\phi}_{N}
\end{array}\right)
$$

onde: $\Delta_{x_{a 1}}=x_{a_{1}}-x_{i}, \Delta_{y_{a 1}}=y_{a_{1}}-y_{i}, \Delta_{x_{b 1}}=x_{b_{1}}-x_{i}, \Delta_{y_{b 1}}=y_{b_{1}}-y_{i}$.

As condições de contorno de Neumann e de Robin podem ser obtidas de forma análoga

\subsubsection{Restrição no contorno para a condição de contorno de Neumann}

Ao longo do contorno $\partial \Omega_{2}$, a solução deve satisfazer a condição de contorno de Neumann, $\frac{\partial \phi}{\partial n}=f_{2}(\vec{x})$.

A restrição de Gauss força a derivada normal para os pontos de Gauss no contorno para a condição especificada. Aplicando a definição de derivada direcional, a condição de 
contorno de Neumann pode ser escrita como:

$$
\frac{\partial \phi}{\partial n}=\frac{\partial \phi}{\partial x} \vec{n}_{x}+\frac{\partial \phi}{\partial y} \vec{n}_{y}=f_{2}(\vec{x})
$$

e para cada ponto de Gauss, $\vec{x}_{g}$, no contorno, segue-se:

$$
\begin{aligned}
f_{2}(\vec{x})= & \left.\vec{n}_{x} \frac{\partial \phi}{\partial x}\right|_{i}+\left.\vec{n}_{y} \frac{\partial \phi}{\partial y}\right|_{i}+\left.\left(\vec{n}_{x}\left(y_{g}-y_{i}\right)\right) \frac{\partial^{2} \phi}{\partial x^{2}}\right|_{i}+\left.\left(\vec{n}_{x}\left(y_{g}-y_{i}\right)+\vec{n}_{y}\left(x_{g}-x_{i}\right)\right) \frac{\partial^{2} \phi}{\partial x \partial y}\right|_{i}+ \\
& \left.\left(\vec{n}_{y}\left(x_{g}-x_{i}\right)\right) \frac{\partial^{2} \phi}{\partial y^{2}}\right|_{i}+\cdots
\end{aligned}
$$

que será acrescentado ao problema mínimos quadrados, resultando no sistema linear

$$
\left(\begin{array}{ccccccc}
1 & \bar{x}_{i} & \bar{y}_{i} & \bar{x}^{2}{ }_{i} & \overline{x y}_{i} & \bar{y}^{2}{ }_{i} & \cdots \\
0 & \left.\vec{n}_{x}\right|_{a_{2}} & \left.\vec{n}_{y}\right|_{a_{2}} & 2 \vec{n}_{x} \Delta x_{a_{2}} & \vec{n}_{x} \Delta x_{a_{2}}+ & \vec{n}_{y} \Delta y_{a_{2}} & \cdots \\
& & & & \vec{n}_{y} \Delta y_{a_{2}} & & \\
0 & \left.\vec{n}_{x}\right|_{b_{2}} & \left.\vec{n}_{y}\right|_{b_{2}} & 2 \vec{n}_{x} \Delta x_{b_{2}} & \vec{n}_{x} \Delta x_{b_{2}}+ & \vec{n}_{y} \Delta y_{b_{2}} & \cdots \\
& & & & \vec{n}_{y} \Delta y_{b_{2}} & & \\
\hline w_{i 1} & w_{i 1} \hat{x}_{i 1} & w_{i 1} \hat{y}_{i 1} & w_{i 1} \hat{x}_{i 1}^{2} & w_{i 1} \widehat{x y}_{i 1} & w_{i 1} \hat{y}_{i 1}^{2} & \cdots \\
w_{i 2} & w_{i 2} \hat{x}_{i 2} & w_{i 2} \hat{y}_{i 2} & w_{i 2} \hat{x}_{i 2}^{2} & w_{i 2} \widehat{x y}_{i 2} & w_{i 2} \hat{y}_{i 2}^{2} & \cdots \\
w_{i 3} & w_{i 3} \hat{x}_{i 3} & w_{i 3} \hat{y}_{i 3} & w_{i 3} \hat{x}_{i 3}^{2} & w_{i 3} \widehat{x y}_{i 3} & w_{i 3} \hat{y}_{i 3}^{2} & \cdots \\
\vdots & \vdots & \vdots & \vdots & \vdots & \vdots & \ddots \\
w_{i N} & w_{i 4} \hat{x}_{i N} & w_{i N} \hat{y}_{i N} & w_{i N} \hat{x}_{i N}^{2} & w_{i N} \widehat{x y}_{i N} & w_{i N} \hat{y}_{i N}^{2} & \cdots
\end{array}\right)\left(\begin{array}{c}
\phi \\
\frac{\partial \phi}{\partial x} \\
\frac{\partial \phi}{\partial y} \\
\frac{\partial^{2} \phi}{\partial x^{2}} \\
\frac{\partial^{2} \phi}{\partial x \partial y} \\
\frac{\partial^{2} \phi}{\partial y^{2}} \\
\vdots \\
\frac{\partial^{n} \phi}{\partial x^{n}}
\end{array}\right)_{i}=\left(\begin{array}{c}
\bar{\phi}_{i} \\
f_{2}\left(\vec{x}_{a}\right) \\
f_{2}\left(\vec{x}_{b}\right) \\
\hline w_{i 1} \bar{\phi}_{1} \\
w_{i 2} \bar{\phi}_{2} \\
w_{i 3} \bar{\phi}_{3} \\
\vdots \\
w_{i N} \bar{\phi}_{N}
\end{array}\right)
$$

onde: $\Delta_{x_{a 2}}=x_{a_{2}}-x_{i}, \Delta_{y_{a 2}}=y_{a_{2}}-y_{i}, \Delta_{x_{b 2}}=x_{b_{2}}-x_{i}, \Delta_{y_{b 2}}=y_{b_{2}}-y_{i}$.

\subsubsection{Restrição no contorno para condição de contorno de Robin}

A restrição, neste caso, pode ser obtida através das equações de restrição para os pontos de Gauss do volume de controle adjacente ao contorno para as condições de contorno de Dirichlet e Neumann acopladas, combinando os termos apropriados obtendo uma equação geral da forma $\alpha \phi(\vec{x})+\beta \frac{\partial \phi}{\partial n}(\vec{x})=f_{3}(\vec{x})$, onde $\alpha$ e $\beta$ são constantes para obter uma nova equação de restrição, resultando em:

$$
\begin{gathered}
\alpha \phi\left(\vec{x}_{g}\right)+\beta \frac{\partial \phi\left(\vec{x}_{g}\right)}{\partial n}=h\left(\vec{x}_{g}\right)=\alpha+\left(\alpha\left(x_{g}-x_{i}\right)+\beta \hat{n}_{x}\right) \frac{\partial \phi}{\partial x}+\left(\alpha\left(y_{g}-y_{i}\right)+\beta \hat{n}_{y}\right) \frac{\partial \phi}{\partial y}+ \\
\left(2 \alpha\left(x_{g}-x_{i}\right)^{2}+\beta\left(x_{g}-x_{i}\right) \hat{n}_{x}\right) \frac{\partial^{2} \phi}{\partial x^{2}}+\left(\alpha\left(x_{g}-x_{i}\right)\left(y_{g}-y_{i}\right)+\right. \\
\left.\left.\beta\left(y_{g}-y_{i}\right) \hat{n}_{x}+\beta x_{g}-x_{i}\right) \hat{n}_{y}\right) \frac{\partial \phi^{2}}{\partial x y}+\left(2 \alpha\left(y_{g}-y_{i}\right)^{2}+\beta\left(y_{g}-y_{i}\right) \hat{n}_{y}\right) \frac{\partial^{2} \phi}{\partial y^{2}} .
\end{gathered}
$$




\subsection{Solução do problema mínimos quadrados restrito}

O sistema obtido (4.7) ou (4.8), geralmente sobredeterminado, deve ser solucionado de tal forma que as equações de restrição sejam resolvidas "exatamente" [70]. Para isto, o método de eliminação de Gauss é utilizado em $r$ linhas, incluindo a restrição média, como pode ser visto na equação (4.9) com 3 linhas de restrição $(r=3)$.

$$
\left(\begin{array}{ccccccc}
c_{1,1} & c_{1,2} & c_{1,3} & c_{1,4} & c_{1,5} & \cdots & c_{1, n} \\
c_{2,1} & c_{2,2} & c_{2,3} & c_{2,4} & c_{2,5} & \cdots & c_{2, n} \\
c_{3,1} & c_{3,2} & c_{3,3} & c_{3,4} & c_{3,5} & \cdots & c_{3, n} \\
\hline x_{1,1} & x_{1,2} & x_{1,3} & x_{1,4} & x_{1,5} & \cdots & x_{1, n} \\
x_{2,1} & x_{2,2} & x_{2,3} & x_{2,4} & x_{2,5} & \cdots & x_{2, n} \\
\vdots & \vdots & \vdots & \vdots & \vdots & \vdots & \vdots \\
x_{m, 1} & x_{m, 2} & x_{m, 3} & x_{m, 4} & x_{m, 5} & \cdots & x_{m, n}
\end{array}\right)\left(\begin{array}{c}
\phi \\
\frac{\partial \phi}{\partial x} \\
\frac{\partial \phi}{\partial y} \\
\frac{\partial^{2} \phi}{\partial x^{2}} \\
\frac{\partial^{2} \phi}{\partial x \partial y} \\
\vdots \\
\frac{\partial^{n} \phi}{\partial y^{n}}
\end{array}\right)_{i}=\left(\begin{array}{c}
C_{1} \\
C_{2} \\
C_{3} \\
\hline X_{1} \\
X_{2} \\
\vdots \\
X_{m}
\end{array}\right)
$$

Na equação (4.9), os c's representam as equações de restrição e os x's representam os demais elementos da matriz mínimos quadrados.

Para cada linha $i, \mathrm{i}=[1, \cdots, \mathrm{r}]$, o método consisite em:

1. determinar o coeficiente de maior valor na linha $i$ e dividir os elementos desta linha por este termo, inclusive o lado direito do sistema linear;

2. procurar a coluna com o maior coeficiente e passá-lo para a coluna $i$. Isto tem o efeito de mudar as colunas correspondentes do vetor solução;

3. A nova coluna $i$, agora contendo o elemento 1 e a linha $i$ é, então reduzida usando eliminação de Gauss da linha $(i+1)$ em diante.

O resultado é uma matriz mínimos quadrados parcialmente reduzida, equação (4.10). O algoritmo mínimos quadrados com uso da transformação de Householder [81] é, então aplicado para a matriz que calcula os termos desconhecidos do polinômio de reconstrução para a aproximação da solução no volume de controle com a restrição apropriada. 


$$
\left(\begin{array}{ccccccc}
1 & \mathbf{d}_{\mathbf{1 , 4}} & d_{1,3} & \mathbf{d}_{\mathbf{1 , 2}} & d_{1,5} & \cdots & d_{1, n} \\
0 & \mathbf{1} & d_{2,3} & \mathbf{d}_{\mathbf{2 , 2}} & d_{2,5} & \cdots & d_{2, n} \\
0 & \mathbf{0} & 0 & \mathbf{d}_{\mathbf{3}, \mathbf{2}} & d_{3,5} & \cdots & d_{3, n} \\
\hline 0 & \mathbf{0} & 0 & \mathbf{y}_{\mathbf{1}, \mathbf{2}} & y_{1,5} & \cdots & y_{1, n} \\
0 & \mathbf{0} & 0 & \mathbf{y}_{\mathbf{2}, \mathbf{2}} & y_{2,5} & \cdots & y_{2, n} \\
\vdots & \vdots & \vdots & \vdots & \vdots & \vdots & \vdots \\
0 & \mathbf{0} & 0 & \mathbf{y}_{\mathbf{m}, \mathbf{2}} & y_{m, 5} & \cdots & y_{m, n}
\end{array}\right)\left(\begin{array}{c}
\phi \\
\frac{\partial \phi^{2} \phi}{\partial \mathbf{x}^{2}} \\
\frac{\partial \phi}{\partial y} \\
\frac{\partial \phi}{\partial \mathbf{x}} \\
\frac{\partial^{2} \phi}{\partial x \partial y} \\
\vdots \\
\frac{\partial^{n} \phi}{\partial y^{n}}
\end{array}\right)_{i}=\left(\begin{array}{c}
D_{1} \\
D_{2} \\
D_{3} \\
Y_{1} \\
Y_{2} \\
\vdots \\
Y_{n}
\end{array}\right)
$$

A equação (4.10) representa (4.9) após aplicar o método de eliminação de Gauss com pivoteamento parcial, onde as colunas 2 e 4 foram trocadas. Os d's representam as equações de restrição trocadas e os y's representam os demais elementos da matriz mínimos quadrados alterada.

Segundo [70], o problema mínimos quadrados também pode ser resolvido através do uso de equações normais com um pouco mais de trabalho. A matriz com restrição obtida (4.10), neste caso, não é usada. Em seu lugar, o algoritmo mínimos quadrados é usado nas equações normais da matriz dadas por $y_{1,2}$ a $y_{n, n}$ para os termos desconhecidos correspondentes. Os termos desconhecidos obtidos são então usados para resolver as equações de restrição para obter os demais coeficientes.

O uso da transformação de Householder [81] fornece uma solução mais precisa para o problema mínimos quadrados do que usando as equações normais, especialmente para matrizes mal-condicionadas. O erro na solução é $\mathrm{O}(2 \mathrm{k})$ usando a transformação de Householder e $\mathrm{O}\left(\epsilon \mathrm{k}^{2}\right)$ para equações normais, onde $\mathrm{k}$ é o número de condição da matriz não-quadrada e $\epsilon$ a precisão de máquina [24]. Isto também implica em grande robustês.

\subsection{Integração e cálculo do fluxo}

Depois da solução ter sido reconstruída na média dos volumes de controle para uma polinomial em cada volume de controle VC, a segunda fase do procedimento da solução é o cálculo do fluxo integral em cada volume de controle. Esta integração deve ser dada com, no mínimo, a mesma ordem de precisão da solução de reconstrução para obter uma solução de alta ordem. No trabalho será usado a quadratura gaussiana em torno do contorno de 
cada volume de controle, que pode ser encontrada com mais detalhes no Apêndice B.

A quadratura gaussiana tem como propriedade que uma quadratura N-pontos ao longo de um segmento de reta tem precisão de ordem $2 \mathrm{~N}$. Desta forma, esquemas de precisão de primeira e segunda ordem $(\mathrm{k}=0,1)$ usa $\mathrm{N}=1$, e $3^{a}$ e $4^{a}$ ordem $(\mathrm{k}=2,3)$ usa $\mathrm{N}=2$.

Para ilustrar o processo, será descrito o algoritmo de solução da equação de advecçãodifusão em 2D (4.11), onde as componentes da velocidade de advecção u e v são conhecidas, geralmente em função de x e y e $\kappa$ é o coeficiente de difusão.

$$
\frac{\partial \phi}{\partial x}+\frac{\partial(u \phi)}{\partial x}+\frac{\partial(u \phi)}{\partial y}=\kappa\left(\frac{\partial^{2} \phi}{\partial x^{2}}+\frac{\partial^{2} \phi}{\partial y^{2}}\right)
$$

Usando o Teorema de Gauss (Teorema da Divergência), pode-se escrever para um volume de controle i $\left(V C_{i}\right)$ a equação

$$
\frac{\partial \bar{\phi}}{\partial t}+\underbrace{\int_{\partial V C_{i}}\left(\begin{array}{cc}
u \phi & \kappa \frac{\partial \phi}{\partial x} \\
v \phi & \kappa \frac{\partial \phi}{\partial y}
\end{array}\right) \cdot \hat{n} d l}=0
$$

onde $\hat{n}$ é o vetor normal unitário à face do VC.

Este problema contém ambos os fluxos: advectivos (que requerem o valor da solução) e fluxos difusivos (que requerem o gradiente da solução). Para qualquer ponto ao longo do volume de controle, a solução e seu gradiente podem ambos serem estimados, pois os valores de dois volumes de controle incidentes podem ser diferentes.

Assim, para os fluxos advectivos usa-se uma aproximação upwind,

$$
F\left(\phi\left(x_{j}\right)\right) \cdot n \approx\left(F\left(\phi^{-}\left(x_{j}\right)\right), F\left(\phi^{+}\left(x_{j}\right)\right) \cdot n\right.
$$

onde $\phi^{+}\left(x_{j}\right)$ é a função $\phi$ reconstruída no volume de controle vizinho ao volume de controle i e $\phi^{-}$é $\phi$ reconstruída no volume de controle i. A direção do fluxo é escolhida de acordo com a direção da solução do vetor velocidade. Os termos fluxos advectivos e difusivos do lado direito de (4.13) são avaliados para os pontos de Gauss de acordo com a ordem da reconstrução.

A aproximação upwind de baixa ordem usualmente tem excessiva dissipação numérica levando a uma solução com uma precisão pobre. Os métodos de alta ordem em esquemas upwind são construídos por aproximações reconstruídas para derivadas de alta ordem das 
variáveis da solução em ambos os lados da face do volume de controle, que podem ser usados para extrapolar valores com maior precisão.

Para os fluxos difusivos, avalia-se os gradientes calculados em cada VC no contorno.

O gradiente na face do volume de controle é calculado considerando a média da expressão dos dois volumes de controle incidentes. Pela Figura 4.7 (a) e (b), entre o volume de controle centrado em $\mathbf{0}$ e $\mathbf{1}$, a expressão para o gradiente médio é determinado por seus gradientes usando suas áreas como peso.

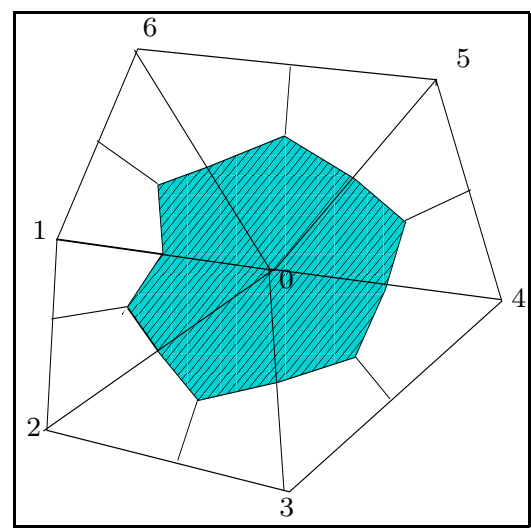

(a)

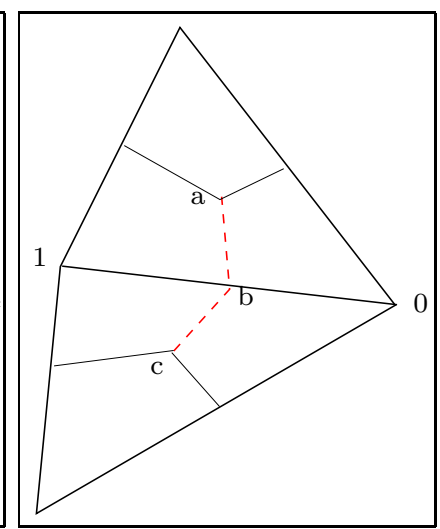

(b)

Figura 4.7: (a) Volume de controle centrado em 0; (b) $\overline{a b}$ e $\overline{b c}$ são as faces do VC para determinar o gradiente médio entre os vértices 0 e 1 .

Ou seja, a equação (4.14) será aplicada nos pontos de Gauss usando os pesos de Gauss e suas respectivas normais para determinar a integral do fluxo. O gradiente médio é obtido a partir das médias ponderadas pela área do volume de controle do gradiente reconstruído em cada volume de controle $\mathbf{0}$ e $\mathbf{1}$, que será aplicado em cada ponto de Gauss da equação (4.15). $A_{0}$ e $A_{1}$ são as áreas do volume de controle $\mathbf{0}$ e $\mathbf{1}$, respectivamente; $\left.\frac{\partial \phi}{\partial x}\right|_{0} ^{R},\left.\frac{\partial \phi}{\partial y}\right|_{0} ^{R}$, $\left.\frac{\partial \phi}{\partial x}\right|_{1} ^{R}$ e $\left.\frac{\partial \phi}{\partial y}\right|_{1} ^{R}$ são os gradientes reconstruídos.

$$
\left(\begin{array}{c}
\frac{\partial \phi}{\partial x} \\
\frac{\partial \phi}{\partial y}
\end{array}\right)=\frac{A_{0}\left(\begin{array}{c}
\left.\frac{\partial \phi}{\partial x}\right|_{0} ^{R} \\
\left.\frac{\partial \phi}{\partial y}\right|_{0} ^{R}
\end{array}\right)+A_{1}\left(\begin{array}{c}
\left.\frac{\partial \phi}{\partial x}\right|_{1} ^{R} \\
\left.\frac{\partial \phi}{\partial y}\right|_{1} ^{R}
\end{array}\right)}{A_{0}+A_{1}}
$$

Assim, a integral do fluxo para cada face do volume de controle é calculada usando 
quadratura gaussiana (4.15) e adicionados ao fluxo integral total.

$$
F l u x=\sum_{k=1}^{N g}\left(\begin{array}{c}
u \phi \kappa \frac{\partial \phi}{\partial x} \\
v \phi \kappa \frac{\partial \phi}{\partial y}
\end{array}\right)\left(x_{g k}\right) \cdot \hat{n}_{g k} W_{g k},
$$

onde $x_{g k}$ são os pontos de Gauss localizados ao longo da face e $W_{g k}$ são os pesos de Gauss correspondentes e a normal $\hat{n}_{g k}$ é a normal unitária à face.

Mantendo a prática padrão: 1 ponto de Gauss por segmento com reconstrução linear, 2 pontos de Gauss para quadrática e 4 pontos de Gauss para cúbica, Tabela 4.2.

\begin{tabular}{|c|c|c|c|}
\hline $\begin{array}{c}\text { Ordem de } \\
\text { Reconstrução }\end{array}$ & $\begin{array}{c}\text { Pontos de } \\
\text { Gauss }\end{array}$ & $\begin{array}{c}\text { Peso de } \\
\text { Gauss }\end{array}$ & $\begin{array}{c}\text { Normal de } \\
\text { Gauss }\end{array}$ \\
\hline \hline 2 & $x_{g}=\frac{L_{1} \frac{x_{a}+x_{b}}{2}+L_{2} \frac{x_{b}+x_{c}}{2}}{L_{1}+L_{2}}$ & $W_{g}=\left|x_{a}-x_{c}\right|$ & $\hat{n}_{g}=\frac{\left(y_{c}-y_{a}, x_{a}-x_{c}\right)}{W_{g}}$ \\
\hline 3 & $x_{g_{1}}=\frac{x_{a}+x_{b}}{2}$ & $W_{g 1}=L_{1}$ & $\hat{n}_{g 1}=\frac{\left(y_{b}-y_{a}, x_{a}-x_{b}\right)}{L_{1}}$ \\
& $x_{g_{2}}=\frac{x_{b}+x_{c}}{2}$ & $W_{g 2}=L_{2}$ & $\hat{n}_{g 2}=\frac{\left(y_{c}-y_{n}, x_{n}-x_{c}\right)}{L_{2}}$ \\
\hline 4 & $x_{g 1}=\frac{x_{a}+x_{b}}{2}+\frac{x_{a}-x_{b}}{2 \sqrt{3}}$ & & $x_{g 2}=\frac{x_{a}+x_{b}}{2}-\frac{x_{a}-x_{b}}{2 \sqrt{3}}$ \\
& $x_{g 3}=\frac{x_{b}-x_{c}}{2}+\frac{x_{b}+x_{c}}{2 \sqrt{3}}$ & $W_{g 3}=W_{g 2}=\frac{L_{1}}{2}$ & $\hat{n}_{g 1}=\hat{n}_{g 2}=\frac{\left(y_{b}-y_{a}, x_{a}-x_{b}\right)}{L_{1}}$ \\
& $x_{g 4}=\frac{L_{b}}{2}-\frac{x_{c}}{2 \sqrt{3}}$ & $\hat{n}_{g 3}=\hat{n}_{g 4}=\frac{\left(y_{c}-y_{b}, x_{b}-x_{c}\right)}{L_{2}}$ \\
\hline
\end{tabular}

Tabela 4.2: Pontos de Gauss, normais e pesos usados no cálculo da integral do fluxo, $L_{1}=\left|x_{a}-x_{b}\right|$ e $L_{2}=\left|x_{b}-x_{c}\right|$.

A integral pode ser resolvida exatamente usando quadratura gaussiana de ordem apropriada ao longo do contorno do VC utilizando os pontos de Gauss, Figura 4.8 (a) e (b).

A integração do fluxo no contorno do domínio é feita de forma análoga. 


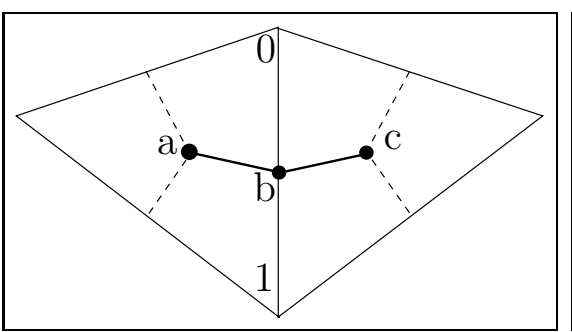

(a)

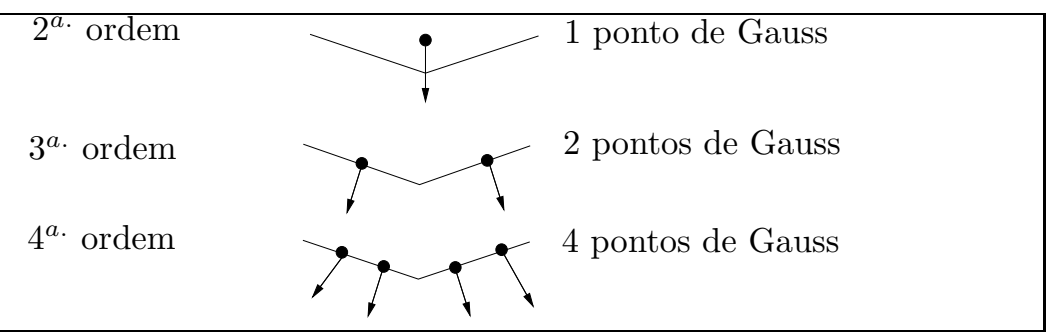

(b)

Figura 4.8: (a) Esquema para determinar os pontos de Gauss no esquema centrado no vértice; (b) número de ponto de Gauss relacionado à ordem de precisão da reconstrução.

\subsection{Evolução temporal}

A equação (4.16) é discretizada para obter o sistema de equações diferenciais ordinárias acoplado

$$
\frac{d \bar{\phi}_{i}}{d t}+\frac{1}{V} R(\phi)=0
$$

onde $R(\phi)$ são os termos da discretização espacial e $\mathrm{V}$ é a área do volume de controle. A equação acima pode ser resolvida tanto por um método explícito como implícito. Aqui será usado um método explícito, ou seja, uma vez calculada a integral do fluxo, a solução é avançada no tempo usando um esquema Runge-Kutta multi-estágios.

Os coeficientes são escolhidos com base na análise do problema modelo, por exemplo, o esquema 5 -estágios com os coeficientes $\alpha_{1}=1 / 15, \alpha_{2}=7 / 45, \alpha_{3}=2 / 7, \alpha_{4}=1 / 2$ e $\alpha_{5}=$ 1, tem boa estabilidade e propriedade de suavização para a equação da onda.

O esquema usa passo no tempo local conforme Ahmad [2], com $\Delta t$ definido para

- difusão

$$
\Delta t=\Delta t_{d i f}=c f l * m_{a t} / a b s(\kappa)
$$

- $\operatorname{advecção~}$

$$
\Delta t=\Delta t_{a d v}=c f l * \frac{\sqrt{a v c_{i} / n t v c_{i}}}{\left\|u^{2}+v^{2}\right\|}
$$

- advecção-difusão

$$
\Delta_{t}=\operatorname{mínimo}\left(\Delta t_{a d v}, \Delta t_{d i f}\right) ;
$$

onde 
$\kappa$ : coeficiente de difusibilidade;

$m_{a t}$ : área da maior célula;

avc: área do volume de controle;

$u$ e $v$ : componentes da velocidade;

ntvc: número de triângulos do volume de controle.

O método de alta ordem para os termos viscosos, que são difusivos, não precisam de tantos cuidados. Gooch e Van Altena [23, 24, 26, 70] mostram que a reconstrução do tipo k-exata podem ser usado para, forçosamente, enfatizar a suavização dos dados na reconstução satisfazendo as condições WENO (condições de Liu, Osher \& Chan). Este esquema permite diminuir a degradação de precisão em regiões onde dados que não são suficientemente suaves existem na reconstrução de alta ordem.

Uma diferença importante entre o presente esquema e os esquemas WENO é o seu comportamento quando um estêncil suave não existe. Enquanto o esquema WENO tem uma deficiência quando não há um estêncil suave, o esquema RMQ automaticamente reduz a ordem de precisão localmente onde há a contaminação da reconstrução. O resultado é um esquema de reconstrução que é bem comportado próximo a múltiplas descontinuidades enquanto conserva a precisão de alta ordem no restante da malha.

Em resumo, o procedimento no esquema de reconstrução mínimos quadrados com alta ordem implementado em linguagem $\mathrm{C}$ neste trabalho consta de:

1. inicializar a média da solução em cada volume de controle;

2. até o tempo final ou o regime estacionário ser atingido:

(a) reconstruir o polinômio em cada volume de controle a partir dos dados;

(b) forçar a condição de contorno;

(c) para cada interface do volume de controle (interior e contorno):

i. calcular o fluxo para os pontos de integração de Gauss;

ii. adicionar a contribuição para a integral de cada volume de controle inicidente na interface;

(d) para cada volume de controle:

i. calcular o termo fonte para os pontos de integração de Gauss; 
ii. integrar sobre o volume de controle;

(e) avançar a solução no tempo.

A Figura 4.9 ilustra o processo descrito.

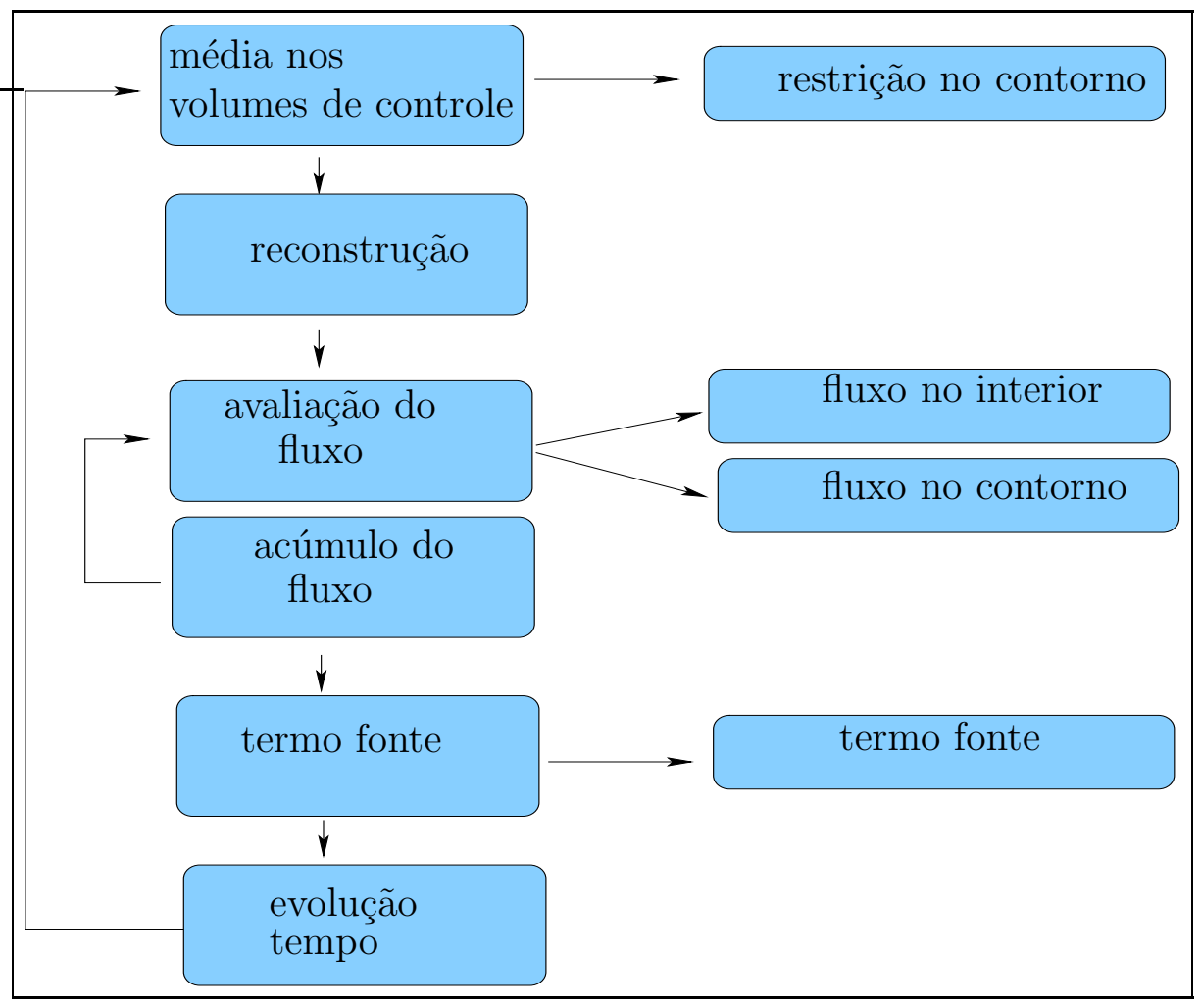

Figura 4.9: Procedimento no esquema de reconstrução mínimos quadrados com alta ordem. 


\section{Verificação do esquema numérico}

Para a verificação da ordem do esquema numérico, alguns testes para a equação de advecção-difusão 2D (e casos particulares) foram realizados. Este capítulo descreve esses testes.

\subsection{Validação do cálculo da integral do fluxo para um volume de controle do interior}

Para verificar a ordem de precisão da reconstrução de alta ordem, Van Altena [70] analisa a ordem de precisão da integral dos fluxos convectivos e difusivos da equação de advecção-difusão, (5.1). Para a equação de Poisson e para a equação de advecção-difusão em um canal retangular, as soluções analíticas são usadas para calcular a divergência do fluxo no domínio.

$$
\frac{\partial \phi}{\partial t}+\frac{\partial(u \phi)}{\partial x}+\frac{\partial(v \phi)}{\partial y}=\kappa\left(\frac{\partial^{2} \phi}{\partial x^{2}}+\frac{\partial^{2} \phi}{\partial y^{2}}\right), \quad \Omega=[0, L] \times[0, h]
$$

O erro no teste da integral do fluxo é usado para calcular as normas do erro global $\left(L_{1}, L_{2}\right.$ e $\left.L_{\infty}\right)$ definidas pela diferença entre a solução exata $\phi$ e a solução aproximada $\phi_{a}$, dada por

$$
E_{i}=\phi\left(x_{i}, y_{i}\right)-\phi_{a}\left(x_{i}, y_{i}\right)
$$


As normas dos erros são definidas por

$$
\begin{aligned}
L_{1} & =\frac{\sum_{i}\left|E_{i}\right|}{N_{i}}, \\
L_{2} & =\sqrt{\frac{\sum_{i} E_{i}^{2}}{N_{i}}}, \\
L_{\infty} & =\max _{i}\left|E_{i}\right|,
\end{aligned}
$$

onde

$L_{1}$ representa o erro absoluto médio no domínio;

$L_{2}$ está relacionada ao desvio padrão $\sigma$ do erro

$$
L_{2}^{2}=L_{1}^{2}+\sigma^{2}
$$

$L_{\infty}$ fornece o máximo valor do erro absoluto no domínio;

$\mathrm{N}$ é o número de volume de controle na malha.

\subsubsection{Equação de difusão - Laplaciano no quadrado unitário}

Para confirmar que a precisão da integral do fluxo calculada pelo método apresentado para o Laplaciano é consistente com a solução analítica fez-se um teste de validação da ordem para a função $\phi(x, y)=\operatorname{sen}(\pi x) \operatorname{sen}(\pi y)$ em uma seqüência de malhas $\mathrm{n} \times \mathrm{n}$, Tabela 5.1 , tendo como domínio o quadrado unitário $\Omega=[0,1] \times[0,1]$.

\begin{tabular}{|c|c|c|c|c|}
\hline Malha & $\mathrm{n} \times \mathrm{n}$ & Vértices & Células & Faces \\
\hline $\mathbf{A}$ & $10 \times 10$ & 145 & 248 & 392 \\
\hline $\mathbf{B}$ & $20 \times 20$ & 535 & 988 & 1522 \\
\hline $\mathbf{C}$ & $40 \times 40$ & 2074 & 3986 & 6059 \\
\hline $\mathbf{D}$ & $80 \times 80$ & 8078 & 15834 & 23911 \\
\hline
\end{tabular}

Tabela 5.1: Malhas utilizadas para a validação da precisão da integral do fluxo do Laplaciano em $\Omega=[0,1] \times[0,1]$. A malha $\mathbf{A}$ pode ser vista na Figura 5.1.

Em (5.1) considerou-se a velocidade de advecção $(u, v)=(0,0)$ e o coeficiente de difusão $\kappa=1$, resultando em $\nabla^{2} \phi=-2 \pi^{2} \phi$. 


\subsection{Validação do cálculo da integral do fluxo para um volume de controle}

do interior

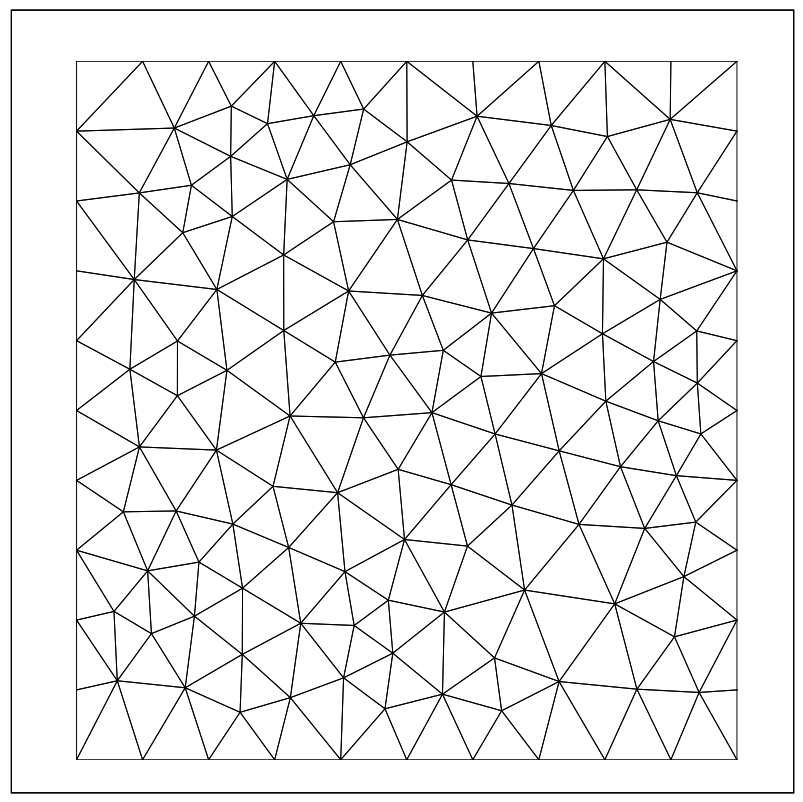

Figura 5.1: Quadrado unitário $\Omega=[0,1] \times[0,1]$ : malha $\mathbf{A}, 145$ vértices, 286 triângulos e 392 faces.

No contorno da região $\Omega=[0,1] \times[0,1]$ considerou-se a função $\phi$, implicando na condição de contorno de Dirichlet homogênea para a equação (5.1).

A Tabela 5.2 mostra as normas dos erros calculados durante os testes para os volumes de controle do interior.

\begin{tabular}{|c|c|c|c|}
\hline $\begin{array}{c}\text { Ordem de } \\
\text { Reconstrução }\end{array}$ & $L_{1}$ & $L_{2}$ & $L_{\infty}$ \\
\hline 2 & 2.04 & 2.02 & 1.55 \\
\hline 3 & 3.38 & 3.32 & 2.82 \\
\hline 4 & 4.08 & 4.02 & 3.52 \\
\hline
\end{tabular}

Tabela 5.2: Ordem de precisão para a integral do fluxo nos VC's internos na equação de Poisson com solução analítica $\phi(x, y)=\operatorname{sen}(\pi x) \operatorname{sen}(\pi y)$.

As taxas de convergência da solução para as normas $L_{\infty}, L_{1}$ e $L_{2}$, são apresentadas nas Figuras 5.2 - 5.4, respectivamente. Os gráficos foram plotados em escala logarítmica, para $2^{a .}, 3^{a .}$ e $4^{a}$ ordem de reconstrução.

A ordem de precisão foi obtida através de uma regressão linear dos pontos no gráfico. 
O coeficiente angular da reta de ajuste é negativo e o seu dobro, em módulo, fornece a ordem de precisão.

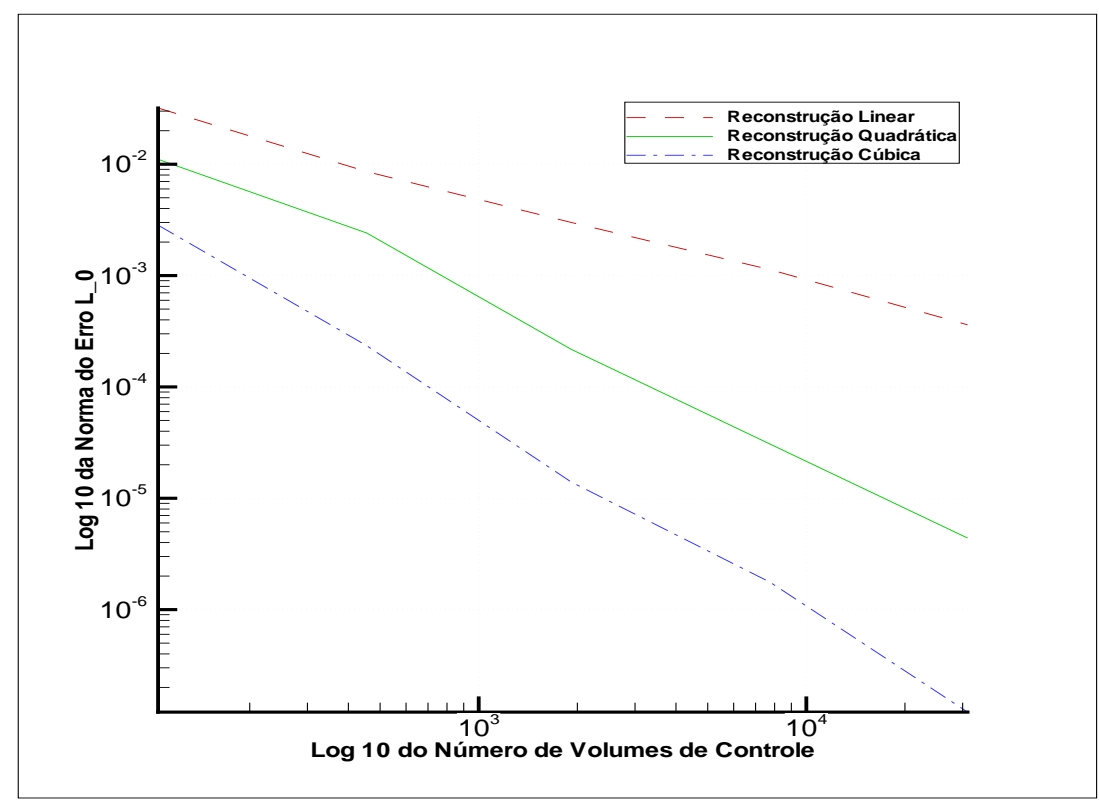

Figura 5.2: Ordem de precisão da reconstrução da função $\phi(x, y)=\operatorname{sen}(\pi x) \operatorname{sen}(\pi y)$ para os volumes de controle do interior: norma $\mathrm{L}_{\infty}$.

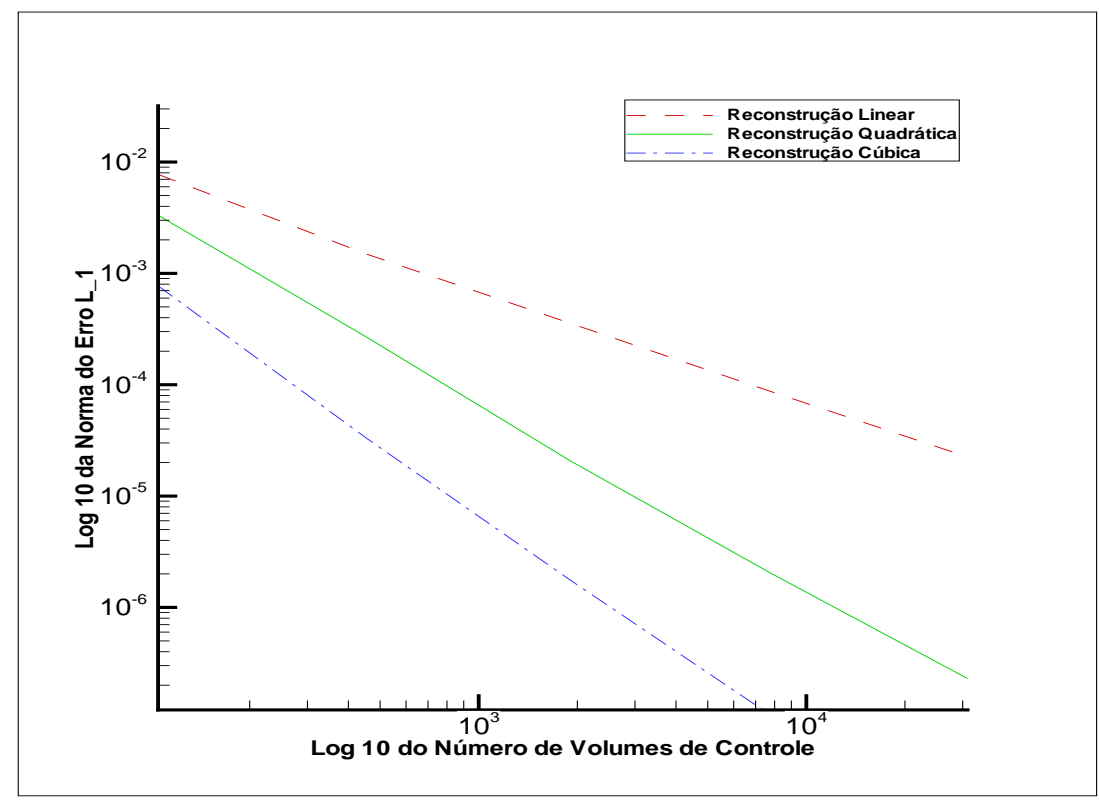

Figura 5.3: Ordem de precisão da reconstrução da função $\phi(x, y)=\operatorname{sen}(\pi x) \operatorname{sen}(\pi y)$ para os volumes de controle do interior: norma $\mathrm{L}_{1}$. 


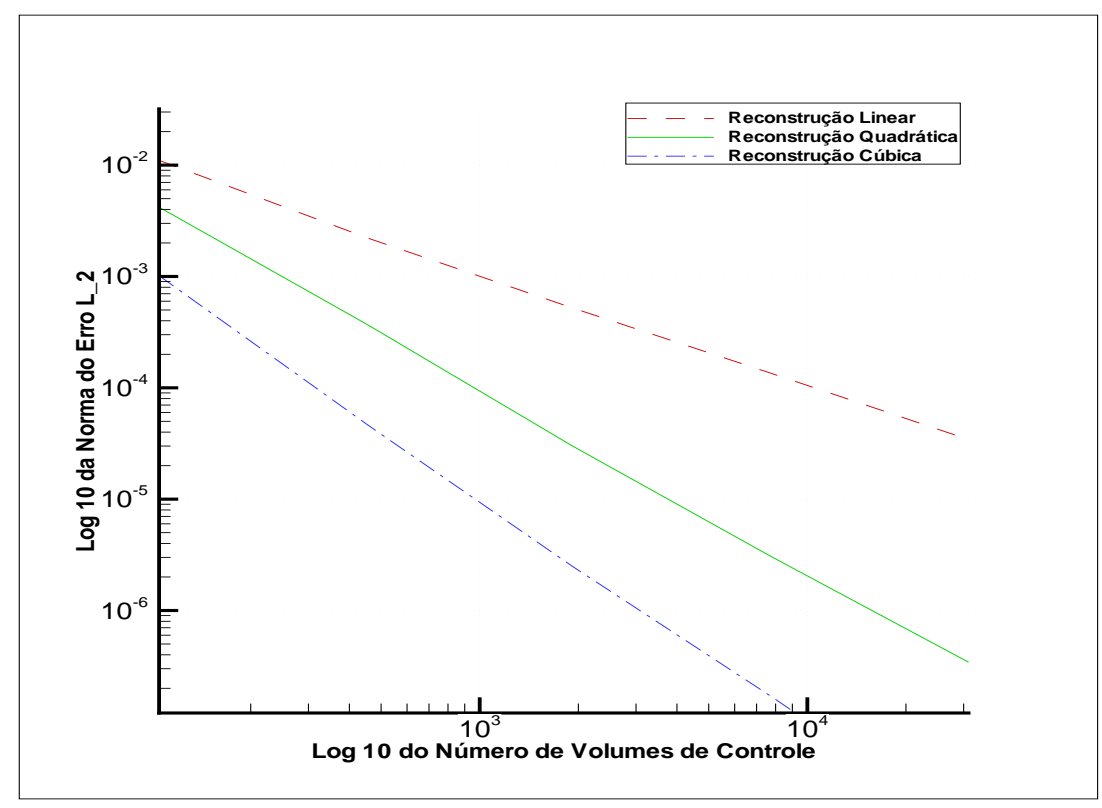

Figura 5.4: Ordem de precisão da reconstrução da função $\phi(x, y)=\operatorname{sen}(\pi x) \operatorname{sen}(\pi y)$ para os volumes de controle do interior: norma $\mathrm{L}_{2}$.

Nas Figuras 5.5 e 5.6 pode-se observar a evolução da aproximação de $\phi$ para uma reconstrução de terceira ordem.

Analisando as Figuras 5.5 e 5.6 pode-se observar a capacidade do algoritmo de representar a solução de uma equação de difusão pura. 


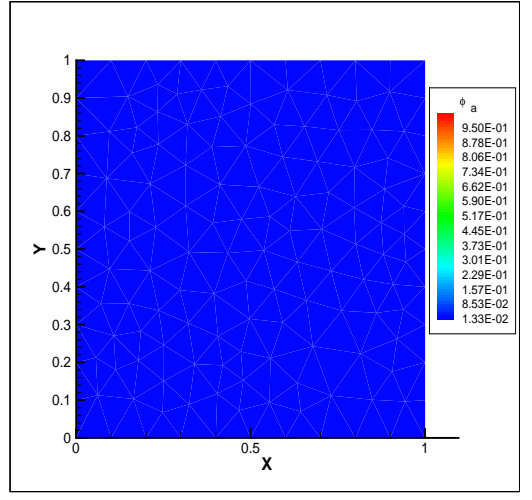

(a)

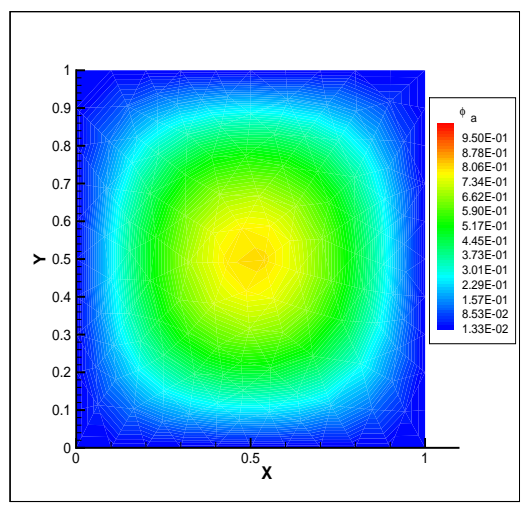

(d)

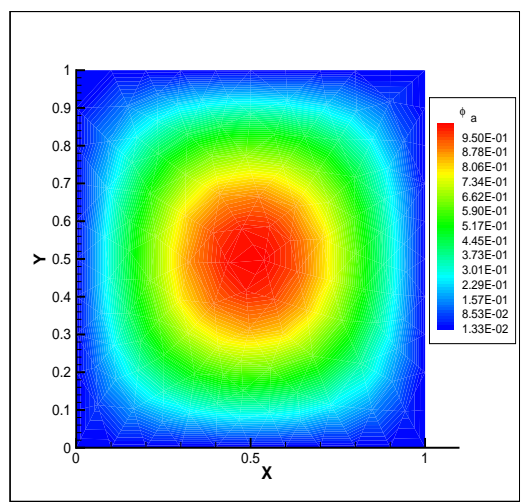

$(\mathrm{g})$

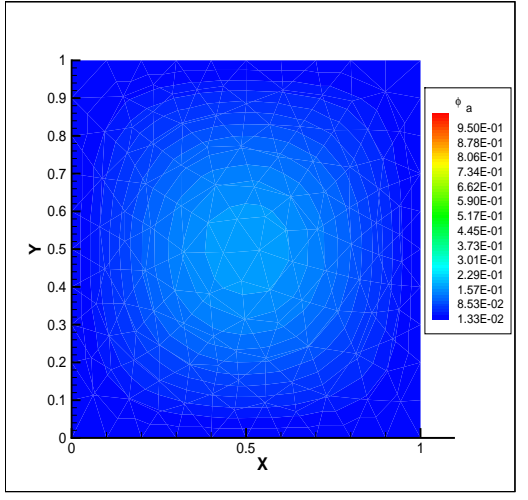

(b)

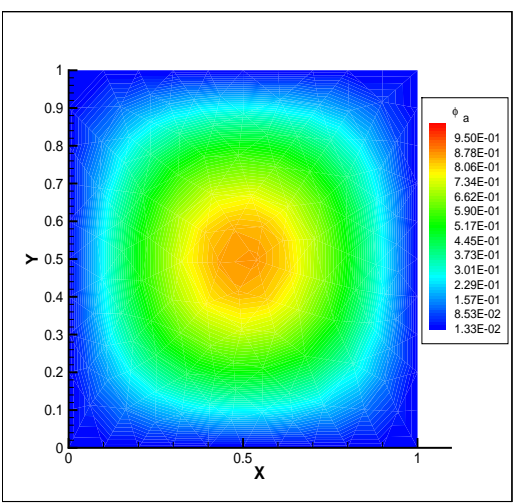

(d)

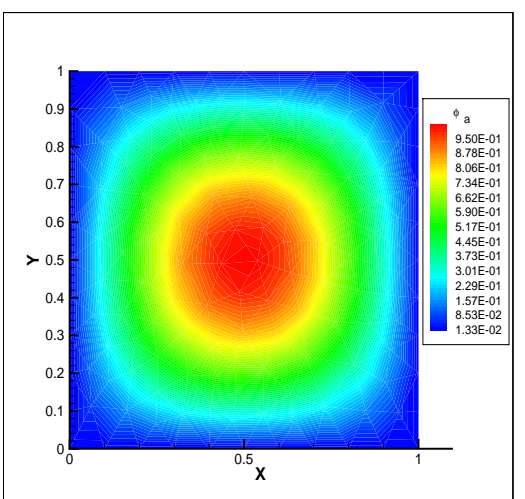

(h)

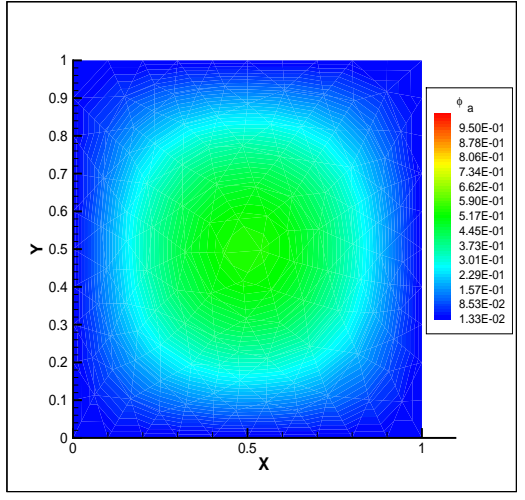

(c)

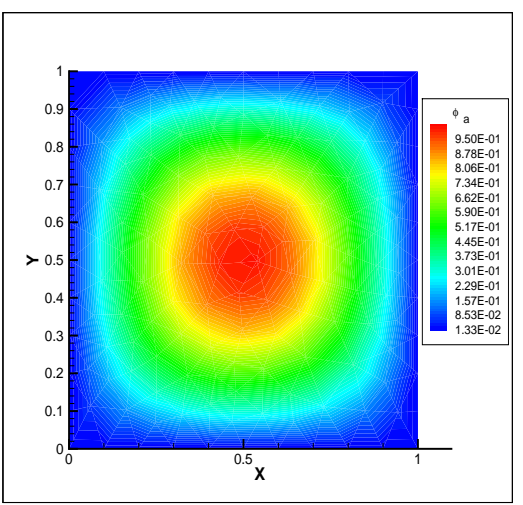

(f)

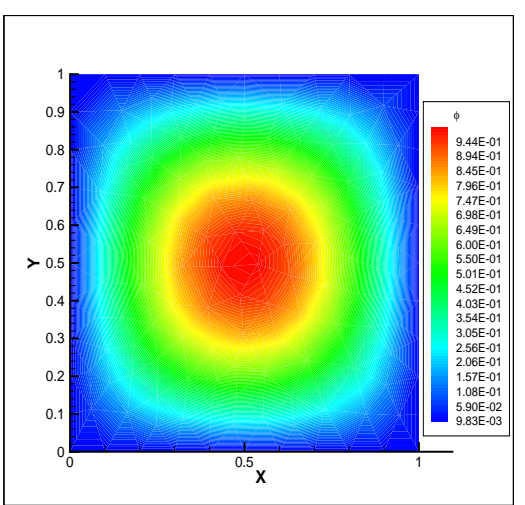

(i)

Figura 5.5: Visualização em 2D da evolução da aproximação numérica de $\phi(x, y)=$ $\operatorname{sen}(\pi x) \operatorname{sen}(\pi y)$ no quadrado unitário, com recontrução de terceira ordem: (a) $\mathrm{t}=1$; (b) $\mathrm{t}=10 ;(\mathrm{c}) \mathrm{t}=40 ;(\mathrm{d}) \mathrm{t}=80 ;(\mathrm{e}) \mathrm{t}=100 ;(\mathrm{f}) \mathrm{t}=200 ;(\mathrm{g}) \mathrm{t}=400 ;(\mathrm{h}) \mathrm{t}=620 ;(\mathrm{i})$ média da solução exata $\phi(x, y)=\operatorname{sen}(\pi x) \operatorname{sen}(\pi y)$. 


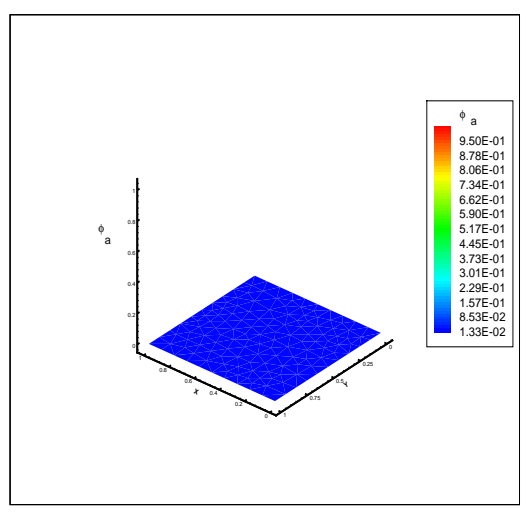

(a)

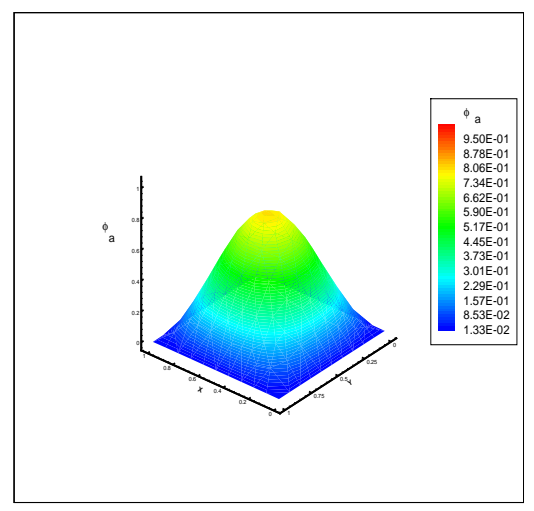

(d)

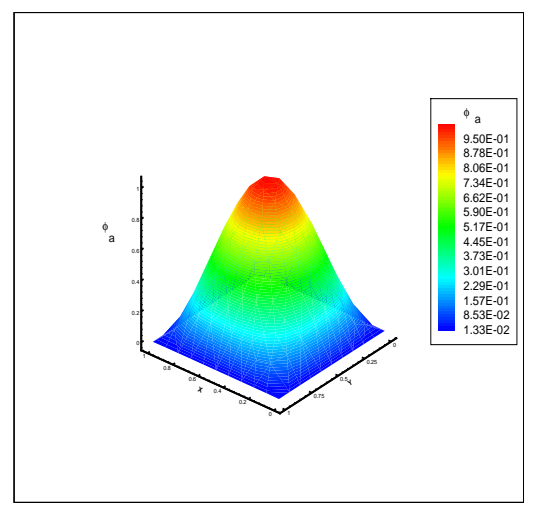

(g)

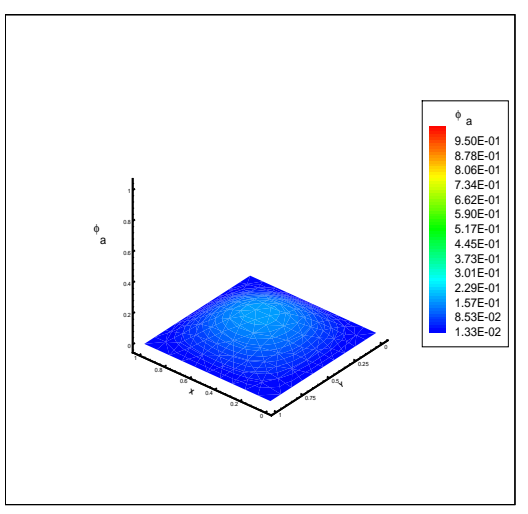

(b)

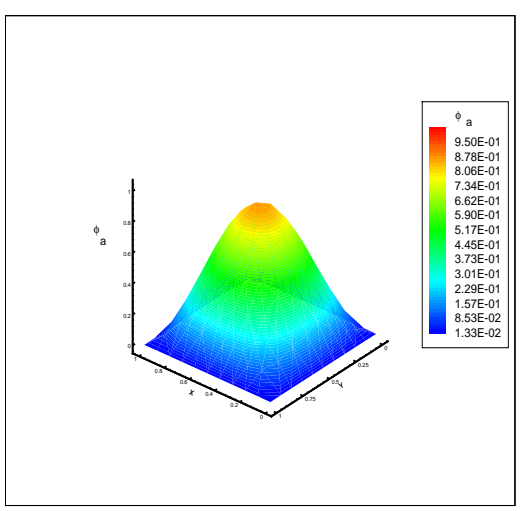

(e)

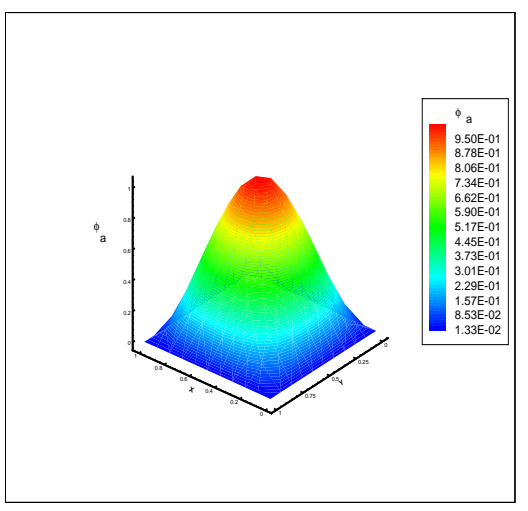

(h)

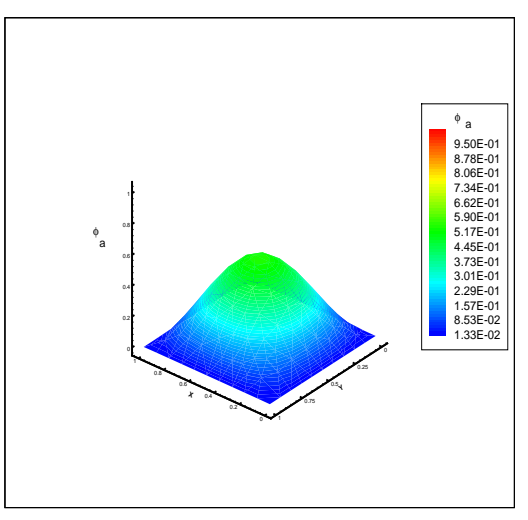

(c)

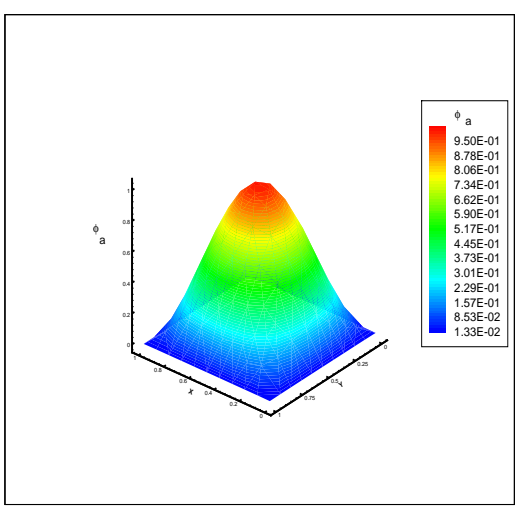

(f)

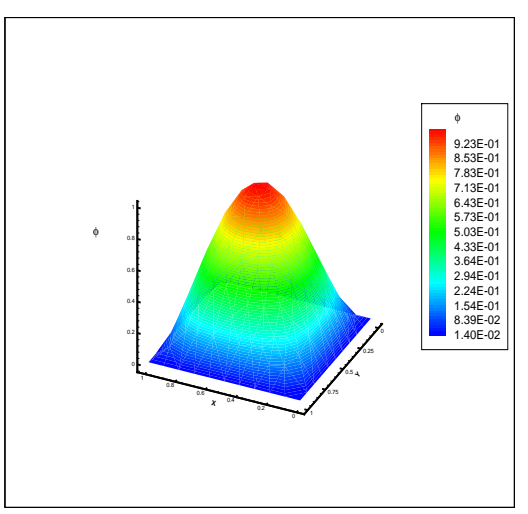

(i)

Figura 5.6: Visualização em 3D da evolução da aproximação numérica de $\phi(x, y)=$ $\operatorname{sen}(\pi x) \operatorname{sen}(\pi y)$ no quadrado unitário, com recontrução de terceira ordem: (a) $\mathrm{t}=1$; (b) $\mathrm{t}=10 ;(\mathrm{c}) \mathrm{t}=40 ;(\mathrm{d}) \mathrm{t}=80 ;(\mathrm{e}) \mathrm{t}=100 ;(\mathrm{f}) \mathrm{t}=200 ;(\mathrm{g}) \mathrm{t}=400 ;(\mathrm{h}) \mathrm{t}=620$; (i) média da solução exata $\phi(x, y)=\operatorname{sen}(\pi x) \operatorname{sen}(\pi y)$. 


\subsubsection{Advecção-difusão no canal retangular}

A equação de advecção-difusão (5.2) é resolvida em [23] no canal retangular de comprimento $L=3$ na direção x e altura unitária $h=1$ na direção y, com constante de velocidade $\omega=\left(u_{0}, 0\right)$, Figura 5.7 (b), e um coeficiente de difusão $\kappa=0.01$.

$$
\frac{\partial \phi}{\partial t}+\frac{\partial(u \phi)}{\partial x}+\frac{\partial(v \phi)}{\partial y}=\kappa\left(\frac{\partial^{2} \phi}{\partial x^{2}}+\frac{\partial^{2} \phi}{\partial y^{2}}\right), \quad \Omega=[0, L] \times[0, h] .
$$

Este teste de ordem envolve três fronteiras com condição de contorno de Dirichlet e uma fronteira com condição de contorno de Neumann. Assim, as condições de contorno são dadas por

$$
\begin{gathered}
\phi(x, 0)=\phi(x, 1)=0, \\
\phi(0, y)=\operatorname{sen}(\pi y), \\
\frac{\partial \phi(L, y)}{\partial x}=0 .
\end{gathered}
$$

E a solução exata é dada por $\phi(x, y)=\operatorname{sen}(\pi y)\left[\frac{r_{2} \mathrm{e}^{\left(r_{1} x+r_{2} L\right)}-r_{1} \mathrm{e}^{\left(r_{1} L+r_{2} x\right)}}{r_{2} \mathrm{e}^{\left(r_{2} L\right)}-r_{1} \mathrm{e}^{\left(r_{1} L\right)}}\right]$, sendo $r_{1,2}=\frac{u_{0}}{2 \alpha} \pm \sqrt{\frac{u_{0}^{2}}{4 \alpha^{2}}+\pi^{2}}$ e pode ser vista na Figura 5.7 (a).

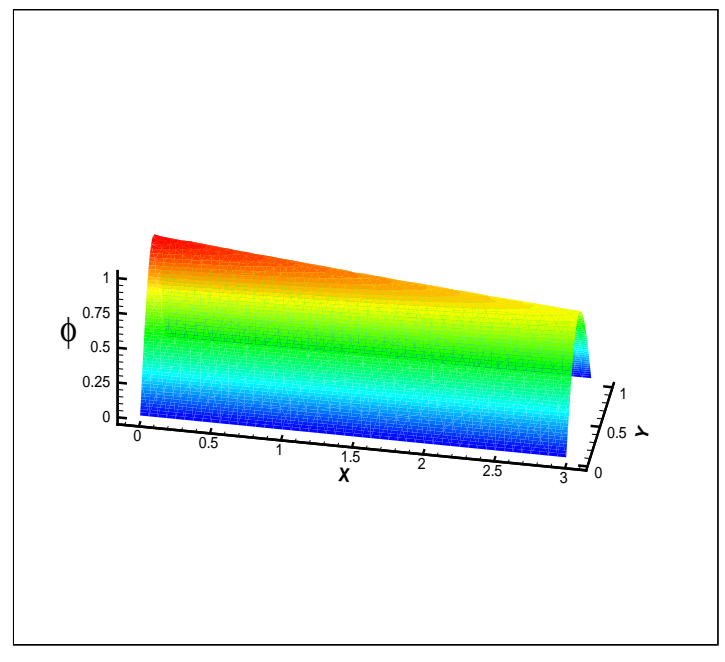

(a)

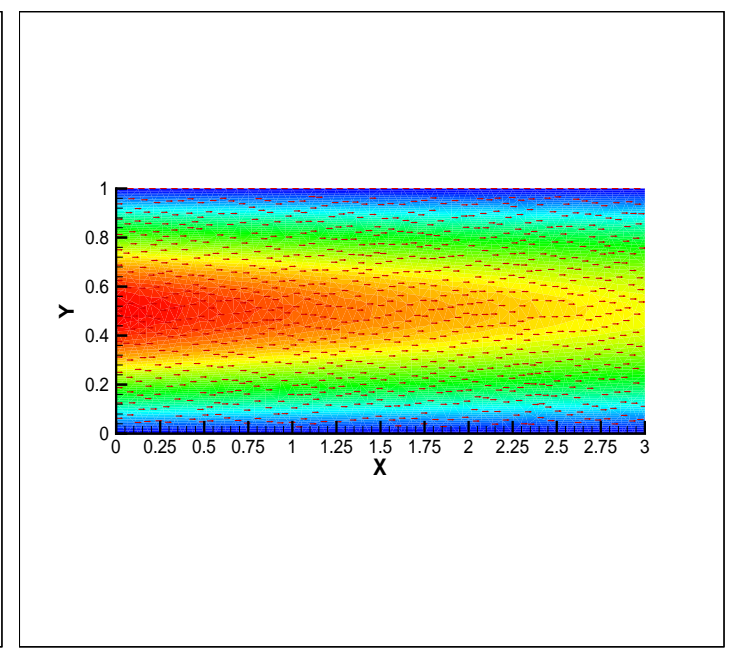

(b)

Figura 5.7: Advecção-difusão em um canal retangular: (a) Função/condição inicial; (b) campo de velocidade $(1,0)$.

O campo de velocidade para este teste é dado por $(u, v)=(1,0)$, Figura 5.7 (b). 


\subsection{Validação do cálculo da integral do fluxo para um volume de controle} do interior

Os testes para a verificação da ordem do método no canal retangular foram feitos utilizando as malhas da Tabela 5.3, $m$ é o número de partições no comprimento $L$ e $n$ é o número de partições na altura $h$.

\begin{tabular}{|c|c|c|c|c|}
\hline Malha & $\mathrm{m} \times \mathrm{n}$ & Vértices & Células & Faces \\
\hline $\mathrm{A}$ & $30 \times 10$ & 411 & 740 & 1150 \\
\hline $\mathrm{B}$ & $60 \times 20$ & 1560 & 2958 & 4517 \\
\hline $\mathrm{C}$ & $120 \times 40$ & 6067 & 11812 & 17878 \\
\hline $\mathrm{D}$ & $240 \times 80$ & 23829 & 47016 & 70844 \\
\hline
\end{tabular}

Tabela 5.3: Malhas utilizadas para a validação da precisão da integral do fluxo no canal retangular: $m$ é o número de partições no comprimento $L$ e $n$ é o número de partições na altura $h$.

Ao aplicar a estratégia de pivoteamento parcial, apresentada por Van Altena [70], percebeu-se que as fronteiras com condição de Neumann, como neste teste, anula a segunda linha da restrição na reconstrução de $2^{a .}$ e $4^{a}$ ordem. A equação (5.7) mostra um exemplo de como seria um sistema para a reconstrução linear em um vértice $i$ com abscissa $L=3$. Na primeira linha da matriz tem-se as médias $\bar{x}_{i}$ e $\bar{y}_{i}$, na segunda e terceira linha as restrições para os pontos de Gauss no contorno.

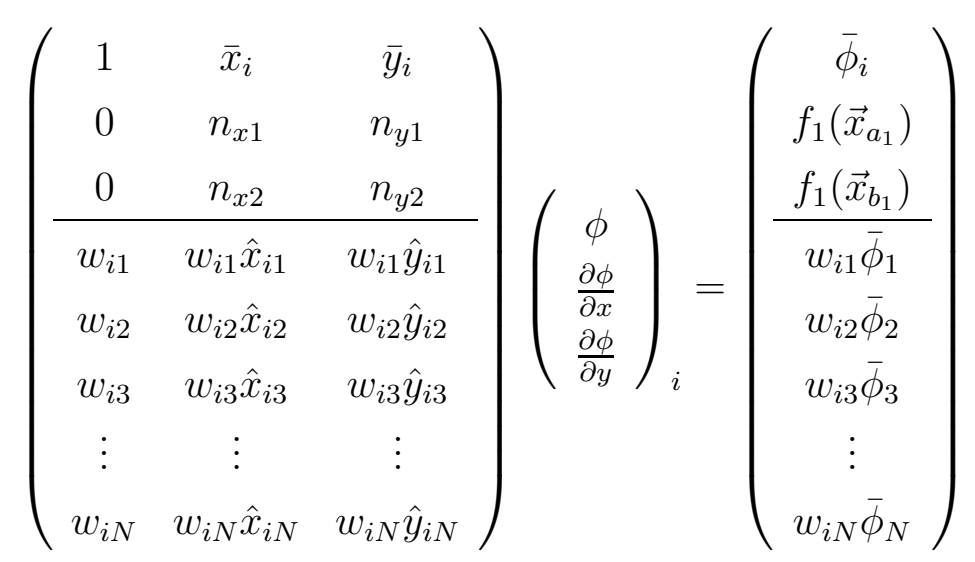

Como o vetor normal unitário $n$ é paralelo ao eixo das abscissas, na fronteira com $\mathrm{L}=3$ os vetores normais $\left(n_{x 1}, n_{y 1}\right)$ e $\left(n_{x 2}, n_{y 2}\right)$ tem a mesma direção e sentido, ou seja, $n_{x 1}=n_{x 2}$ e $n_{y 1}=n_{y 2}$. Assim, quando se aplica o pivoteamento parcial, obtém-se (5.8) com uma 
linha nula.

$$
\left(\begin{array}{ccc}
1 & \bar{x}_{i} & \bar{y}_{i} \\
0 & 1 & 0 \\
\mathbf{0} & \mathbf{0} & \mathbf{0} \\
\hline 0 & 0 & y_{i 1} \\
0 & 0 & y_{i 2} \\
0 & 0 & y_{i 3} \\
\vdots & \vdots & \vdots \\
0 & 0 & y_{i N}
\end{array}\right)\left(\begin{array}{c}
\phi \\
\frac{\partial \phi}{\partial x} \\
\frac{\partial \phi}{\partial y}
\end{array}\right)_{i}=\left(\begin{array}{c}
\bar{\phi}_{i} \\
f_{1}^{\prime}\left(\vec{x}_{a_{1}}\right) \\
\frac{f_{1}^{\prime}\left(\vec{x}_{b_{1}}\right)}{X_{1}} \\
X_{2} \\
X_{3} \\
\vdots \\
X_{N}
\end{array}\right) .
$$

Para contornar este problema, adotou-se nas fronteiras com condição de Neumann a recontrução de terceira ordem.

A Tabela 5.4 apresenta o resultado do teste da reconstrução da ordem, que reflete o esperado, ou seja, na reconstrução de segunda e terceira ordem, a ordem se manteve. Porém, na reconstrução de quarta ordem isto não ocorre, pois nos pontos do interior e sob condição de contorno de Dirichlet, tem-se a reconstrução de quarta ordem e na fronteira com condição de contorno de Neumann tem-se uma reconstrução de terceira ordem, refletindo nos resultados, prevalecendo a menor ordem.

\begin{tabular}{|c|c|c|c|}
\hline $\begin{array}{c}\text { Ordem de } \\
\text { Reconstrução }\end{array}$ & $L_{1}$ & $L_{2}$ & $L_{\infty}$ \\
\hline 2 & 2.01 & 2.00 & 1.78 \\
\hline 3 & 3.45 & 3.33 & 2.55 \\
\hline 4 & 3.82 & 2.91 & 2.02 \\
\hline
\end{tabular}

Tabela 5.4: Ordem de precisão da reconstrução usando as malhas da Tabela 5.3 para a integral do fluxo nos VC's internos na equação de advecção-difusão no canal retangular.

O mesmo pode ocorrer em uma condição de contorno de Robin, $\alpha_{r} \phi(\vec{x})+\beta_{r} \frac{\partial \phi}{\partial n}(\vec{x})=f$, quando $\alpha_{r}=0$.

As taxas de convergência da solução para as normas $L_{\infty}, L_{1}$ e $L_{2}$, são apresentadas nas Figuras 5.8 - 5.10, respectivamente. Os gráficos foram plotados em escala logarítmica, para $2^{a .}, 3^{a}$ e $4^{a}$ ordem de reconstrução. 


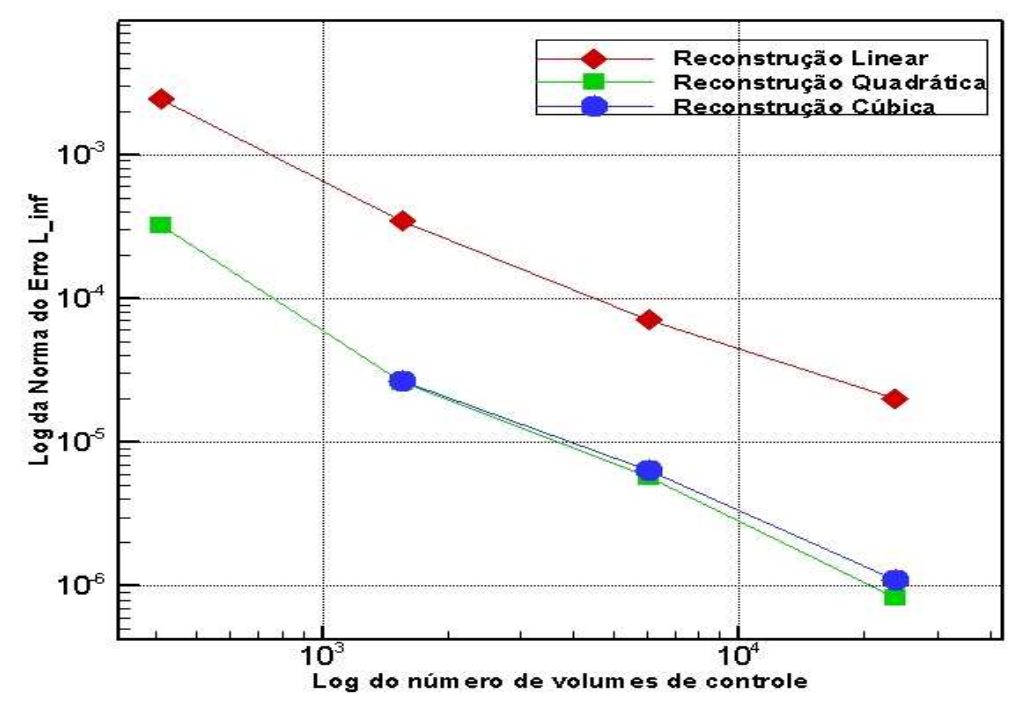

Figura 5.8: Ordem de precisão da reconstrução da integral do fluxo no canal retangular: norma $\mathrm{L}_{\infty}$.

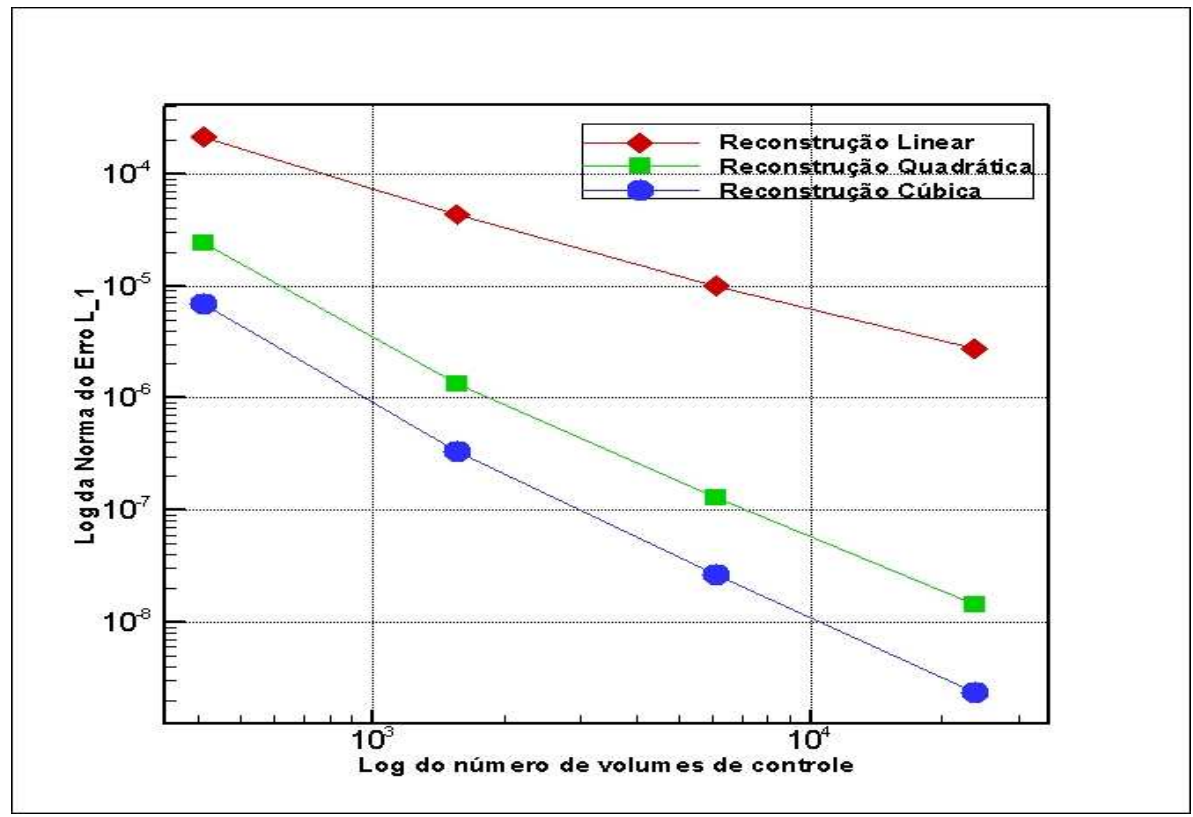

Figura 5.9: Ordem de precisão da reconstrução da integral do fluxo no canal retangular: norma $\mathrm{L}_{1}$. 


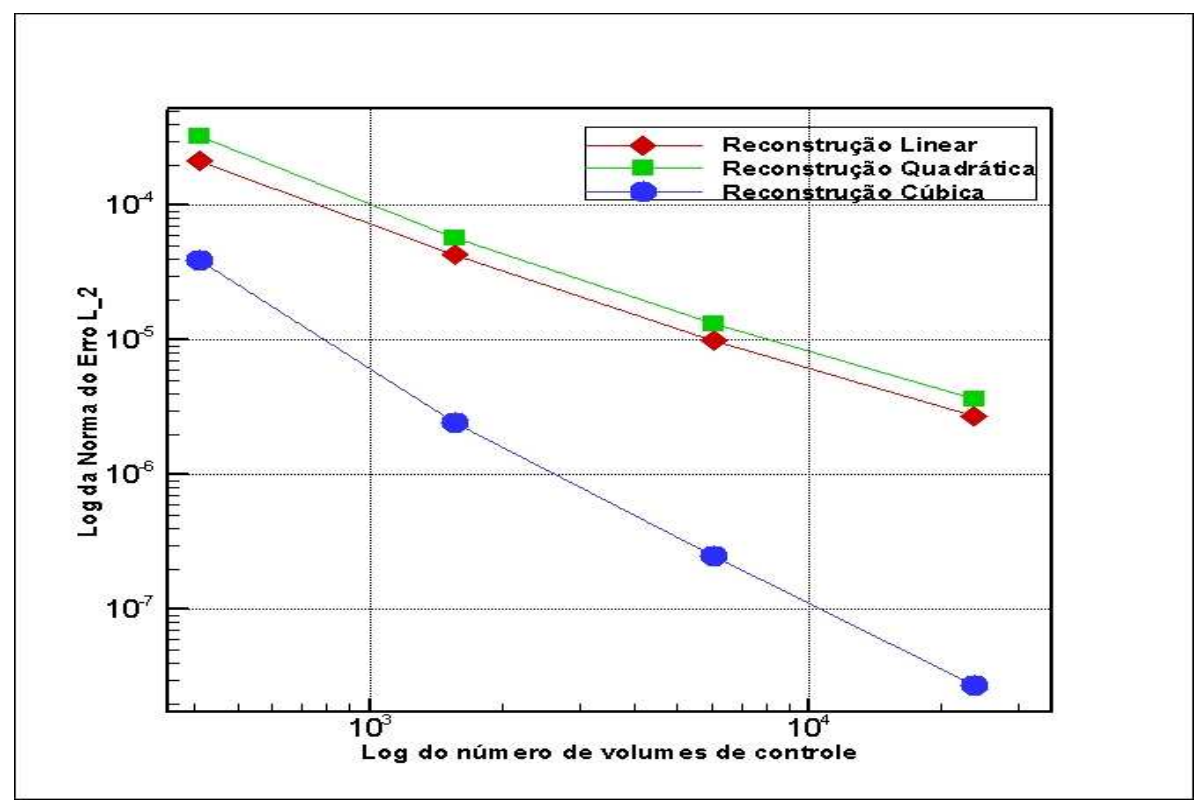

Figura 5.10: Ordem de precisão da reconstrução da integral do fluxo no canal retangular: norma $\mathrm{L}_{2}$.

Assim, conseguiu-se representar com sucesso a solução de uma equação de advecçãodifusão.

\subsection{Equação de advecção - cone de revolução}

Este problema-teste tem como domínio o quadrado $\Omega=[0,2] \times[0,2]$ com 60 partições na direção x e y, respectivamente, num total de 4545 vértices e 8848 triângulos, com condição de contorno homogênea imposta igual a zero em todos os contornos do domínio, $[15,36]$.

A distribuição inicial, Figura 5.11, é especificada por

$$
\phi_{0}(x, y)=\left\{\begin{array}{l}
\cos ^{2}(2 \pi r), \text { se } r=\sqrt{(x-0.5)^{2}+(y-1)^{2}} \leq 0.25 \\
0, \text { caso contrário. }
\end{array}\right.
$$

O campo de escoamento rotacional é definido por $u=-\omega\left(y-y_{0}\right)$ e $v=\omega\left(x-x_{0}\right)$, onde $u(x, y)$ e $v(x, y)$ são as componenetes da velocidade na direção x e y, respectivamente. $\omega=2 \pi$ é a velocidade angular constante e $\left(x_{0}, y_{0}\right)$ é o centro da malha, Figura 5.12. 


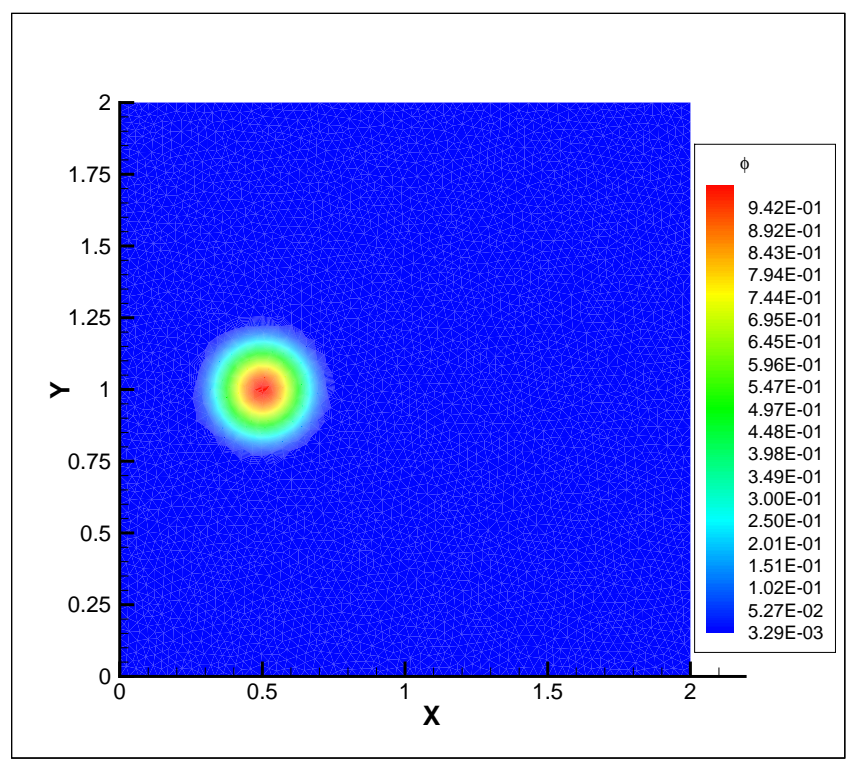

Figura 5.11: Condição inicial para o cone de revolução.

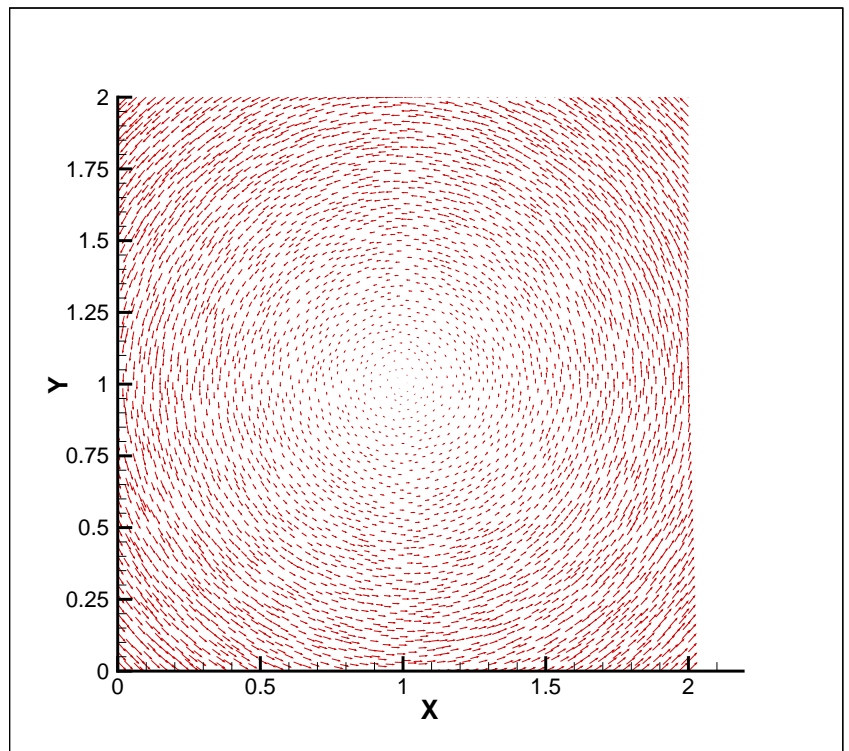

Figura 5.12: Revolução de um cone: campo de velocidade definido por $u=-2 \pi(y-1)$ e $v=2 \pi(x-1)$. 
O passo de tempo é $\Delta t=0.001$, resultando em uma revolução completa após 1000 passos, como é mostrado na Figura 5.13. O mesmo teste pode ser visto, em uma perspectiva 3D, na Figura 5.14.

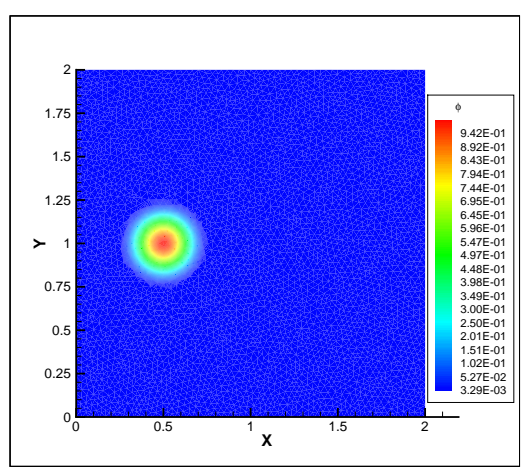

(a)

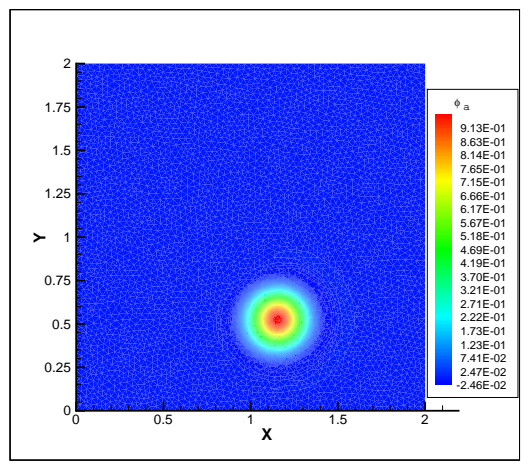

(d)

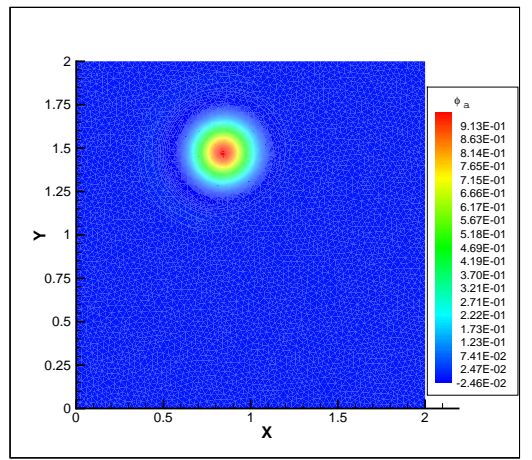

$(\mathrm{g})$

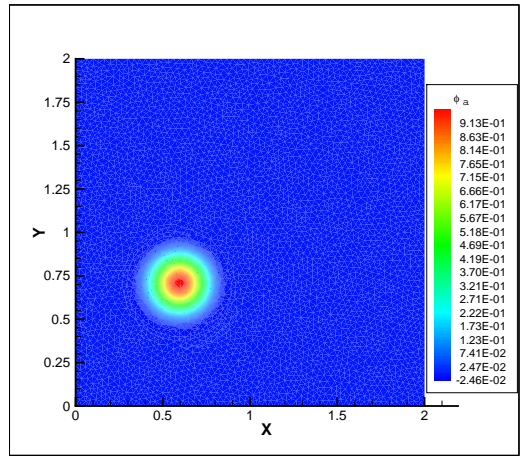

(b)

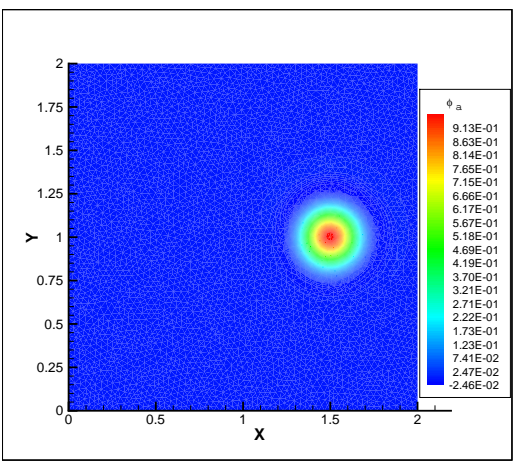

(e)

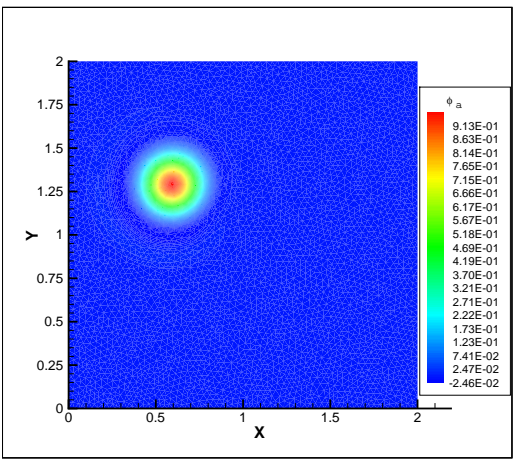

(h)

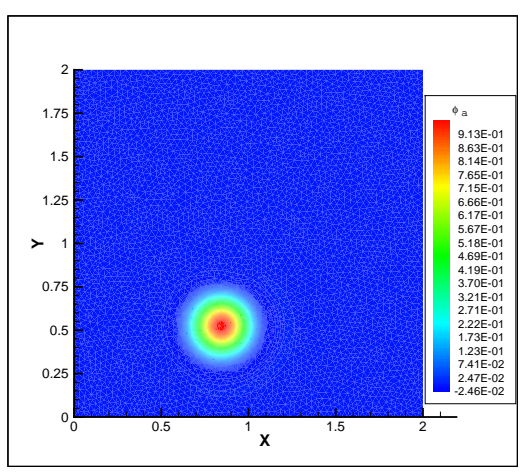

(c)

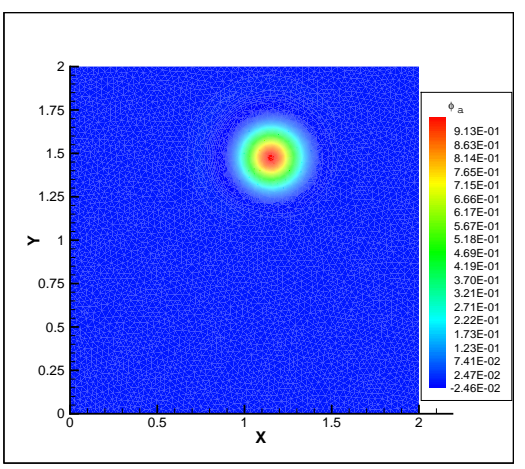

(f)

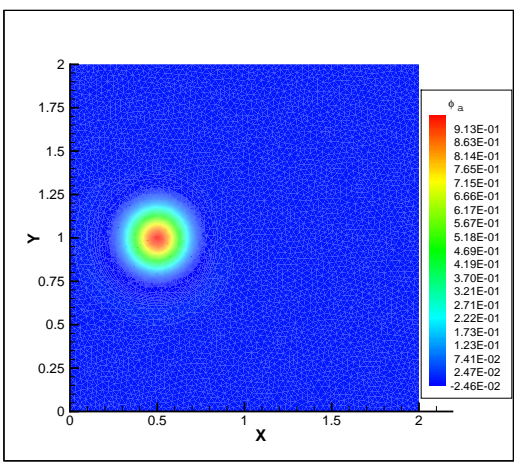

(i)

Figura 5.13: Visualização em 2D da rotação do cone de revolução com precisão de $4^{a}$. ordem, para $\Delta t=0.001$ : (a) distribuição inicial; (b) $\mathrm{t}=100 ;(\mathrm{c}) \mathrm{t}=200 ;$ (d) $\mathrm{t}=300$; (e) $\mathrm{t}=500 ;($ f) $\mathrm{t}=700 ;(\mathrm{g}) \mathrm{t}=800 ;(\mathrm{h}) \mathrm{t}=900 ;$ (i) $\mathrm{t}=1000$. 


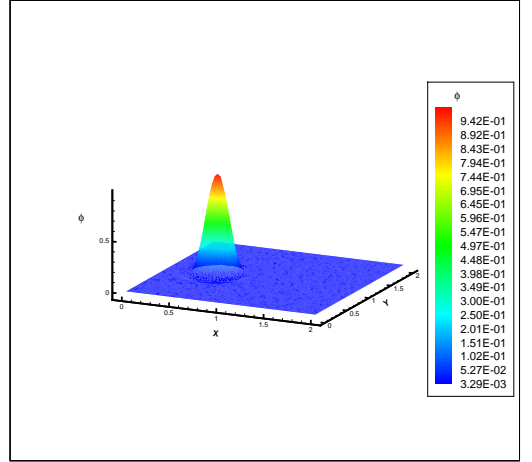

(a)

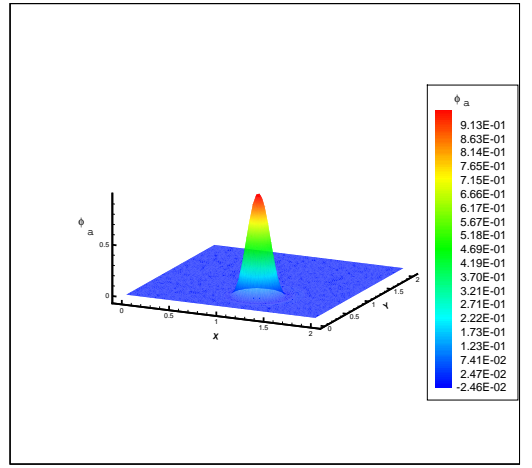

(d)

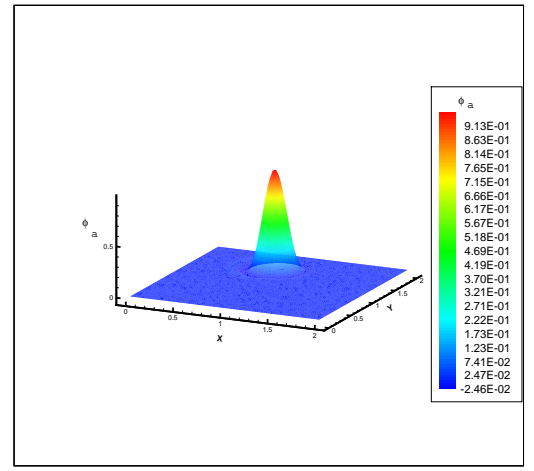

$(\mathrm{g})$

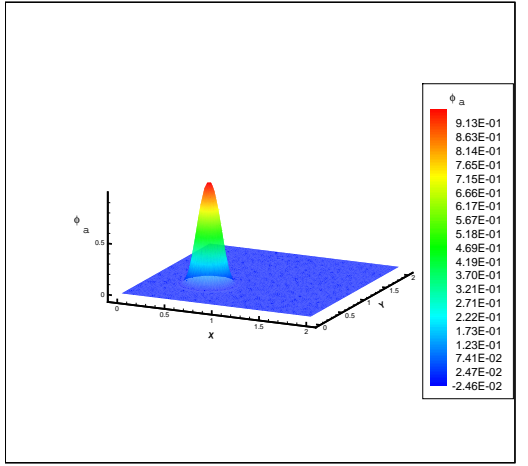

(b)

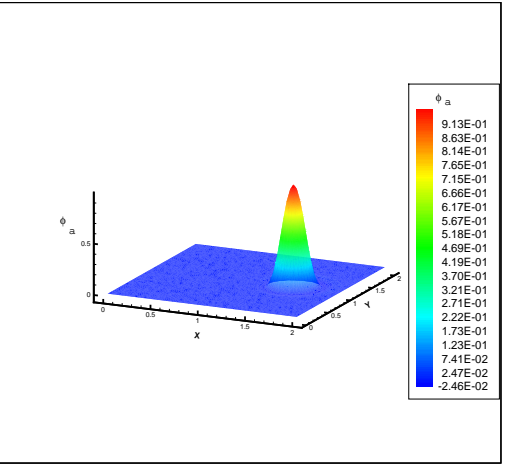

(e)

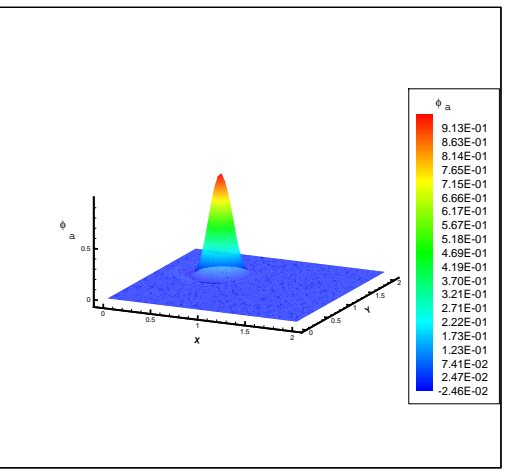

(h)

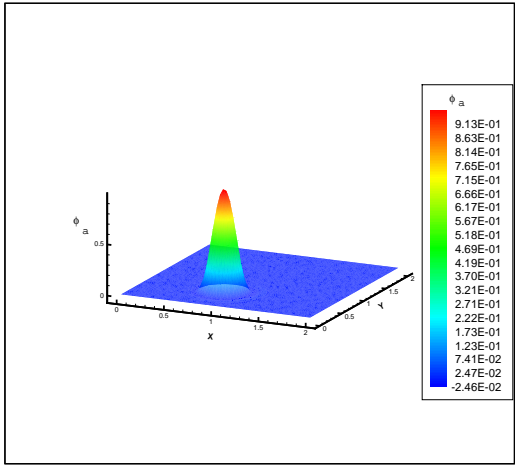

(c)

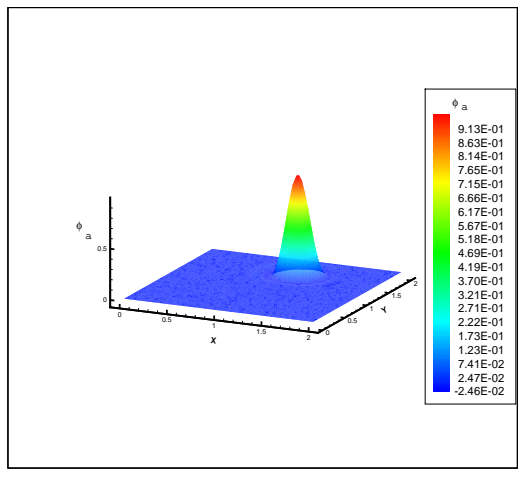

(f)

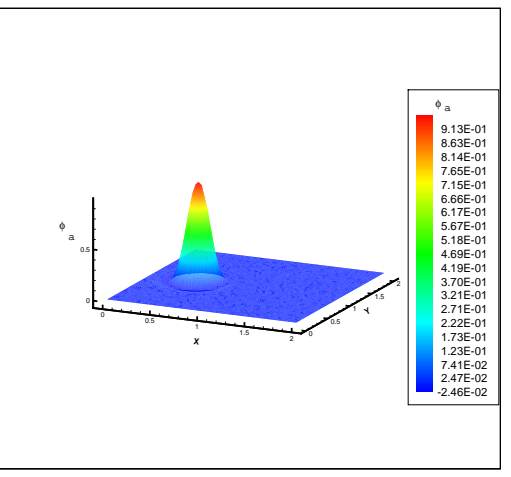

(i)

Figura 5.14: Visualização em 3D da rotação do cone de revolução com precisão de $4^{a}$. ordem, para $\Delta t=0.001$ : (a) distribuição inicial; (b) t $=100 ;(\mathrm{c}) \mathrm{t}=200 ;$ (d) $\mathrm{t}=300$; (e) $\mathrm{t}=500 ;(\mathrm{f}) \mathrm{t}=700 ;(\mathrm{g}) \mathrm{t}=800 ;(\mathrm{h}) \mathrm{t}=900 ;$ (i) $\mathrm{t}=1000$. 
A Figura 5.15 mostra o cone após 5 rotações, comparando-o com uma rotação completa para uma reconstrução de $4^{a}$ ordem e se observa o efeito da dissipação à medida que se aumenta o número de rotações.

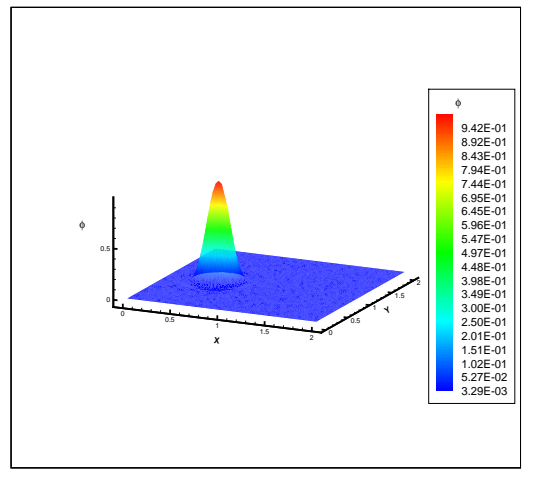

(a)

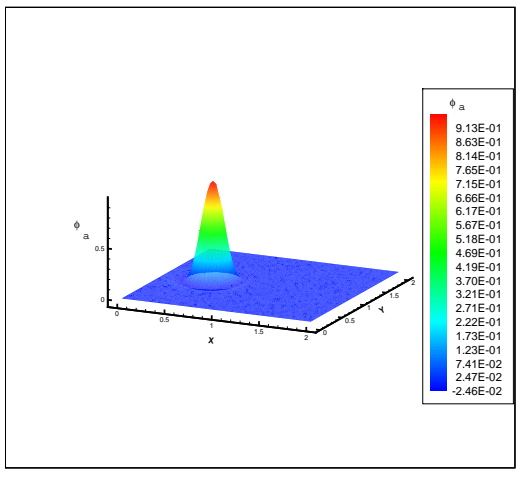

(b)

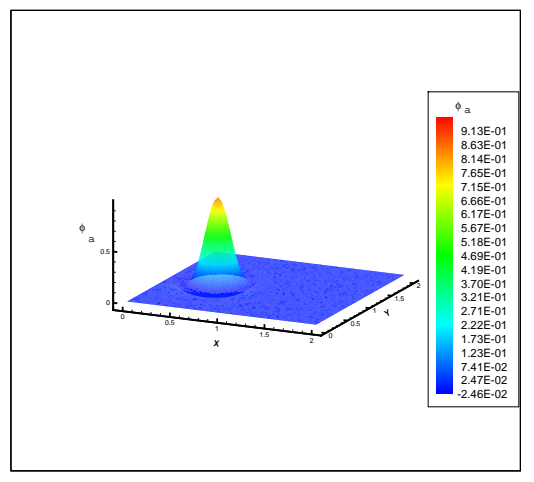

(c)

Figura 5.15: Visualização em 3D do cone de revolução, usando reconstrução da solução com precisão de $4^{a}$ ordem: (a) condição incial (média nos volumes de controle); (b) após uma rotação completa; (b) após 5 rotações completas.

A Figura 5.16 mostra, um corte de perfil no cone de revolução, após uma rotação, comparando os resultados entre as reconstruções da solução de $2^{a}, 3^{a}$ e $4^{a}$ ordem. Notase que a reconstrução de $2^{a}$ ordem apresenta um resultado melhor que o de $3^{a}$ ordem, porém há um atraso na conclusão da rotação.

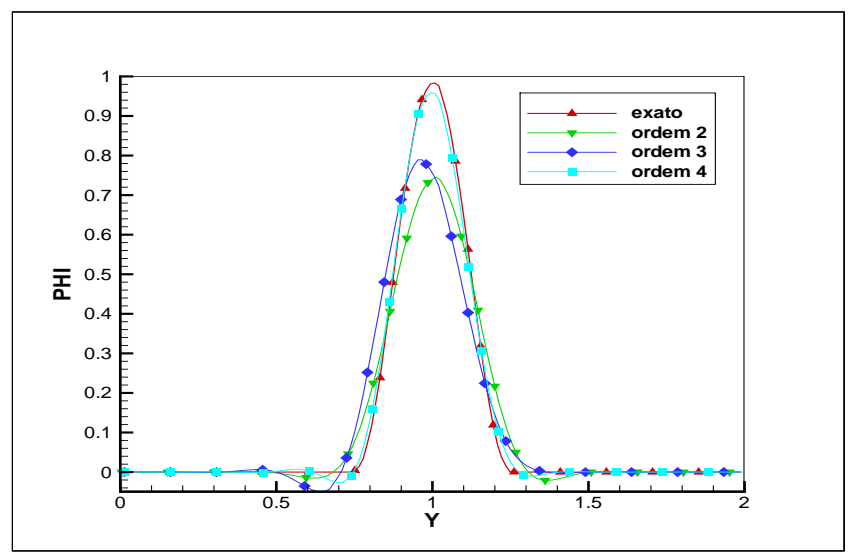

Figura 5.16: Corte de perfil no cone de revolução para $x=0.5$, na comparação após uma rotação, reconstrução da solução de $2^{a}, 3^{a}$ e $4^{a}$ ordem. 
Na Tabela 5.5 tem-se na primeira coluna a ordem do polinômio de reconstrução; na segunda o número de rotações, na terceira os valores máximos atingidos após uma e cinco rotações, na quarta os valores mínimos; na quinta coluna, a norma do máximo e na última coluna o tempo de execução.

E, observa-se que nas soluções numéricas, há uma melhoria da precisão à medida que aumenta-se a ordem de reconstrução, bem como o valor máximo e mínimo após 1 rotação e 5 rotações. Assim como pode observar que o erro é pequeno após 5 rotações.

\begin{tabular}{|c|c|c|c|c|c|}
\hline Ordem & $\begin{array}{c}\text { Número de } \\
\text { rotações }\end{array}$ & $\begin{array}{c}\phi_{a} \\
\text { máx. }\end{array}$ & $\begin{array}{c}\phi_{a} \\
\text { min. }\end{array}$ & $L_{\infty}$ & $\begin{array}{c}\text { Tempo } \\
(\mathrm{s})\end{array}$ \\
\hline \hline \multirow{3}{*}{2} & inicial & 1.0 & 0.0 & $\emptyset$ & $\emptyset$ \\
& 1 & $\mathbf{0 . 7 8 8}$ & -0.045 & $2.82 \mathrm{e}-01$ & 194 \\
& 5 & $\mathbf{0 . 4 8 7}$ & -0.071 & $6.02 \mathrm{e}-01$ & 966 \\
\hline \multirow{3}{*}{3} & inicial & 1,0 & 0.0 & $\emptyset$ & $\emptyset$ \\
& 1 & $\mathbf{0 . 7 3 3}$ & -0.017 & $2.51 \mathrm{e}-01$ & 460 \\
& 5 & $\mathbf{0 . 4 7 3}$ & -0.017 & $5.32 \mathrm{e}-01$ & 2194 \\
\hline \multirow{3}{*}{4} & inicial & 1.0 & 0.0 & $\emptyset$ & $\emptyset$ \\
& 1 & 0.946 & 0.022 & $7.24 \mathrm{e}-02$ & 1765 \\
& 5 & 0.812 & -0.052 & $2.05 \mathrm{e}-01$ & 8808 \\
\hline
\end{tabular}

Tabela 5.5: Resultados obtidos no teste realizado com o cone de revolução.

Para fazer uma análise da ordem de reconstrução $\times$ malha $\times$ tempo, repetiu-se o mesmo teste refinando a malha: 120 partições em cada lado do domínio, num total de 17890 vértices e, também, para 240 partições (71119 vértices) e observou-se que, Tabela 5.6, ao aumentar o refinamento da malha (17890 vértices) com reconstrução linear obtémse um resultado melhor do que em uma malha de 4545 vértices com uma reconstrução cúbica. Análises análogas podem ser vistas nas Tabelas 5.7, 5.8 e nos gráficos do perfil para $\mathrm{x}=0.5$ para as 3 malhas, Figuras $5.17,5.18$ e 5.19 .

Na Figura 5.17 (a) após 5 voltas a segunda ordem apresenta um valor máximo mais próximo de 1.0 que na Figura 5.18 (a), porém a primeira apresenta um atraso ao completar as 5 rotações. E é visível que ao refinar a malha a dissipação se reduz significativamente, sendo mais visível na reconstrução de quarta ordem. 


\begin{tabular}{|c|c|c|c|c|c|}
\hline $\begin{array}{c}\text { Número de } \\
\text { vértices }\end{array}$ & $\begin{array}{c}\text { Número de } \\
\text { revoluções }\end{array}$ & $\begin{array}{c}\phi_{a} \\
\text { máx. }\end{array}$ & $\begin{array}{c}\phi_{a} \\
\text { min. }\end{array}$ & $L_{\infty}$ & $\begin{array}{c}\text { Tempo } \\
(\mathrm{s})\end{array}$ \\
\hline \hline \multirow{3}{*}{4545} & inicial & 1.0 & 0,0 & $\emptyset$ & $\emptyset$ \\
& 1 & 0.788 & -0.045 & $2.82 \mathrm{e}-01$ & 194 \\
& 5 & 0.487 & -0.071 & $6.02 \mathrm{e}-01$ & 966 \\
\hline \multirow{3}{*}{17890} & inicial & 1.0 & 0.0 & $\emptyset$ & $\emptyset$ \\
& 1 & 0.925 & -0.029 & $1.08 \mathrm{e}-01$ & 1131 \\
& 5 & 0.805 & -0.086 & $3.22 \mathrm{e}-01$ & 4418 \\
\hline \multirow{3}{*}{71119} & inicial & 1.0 & 0.0 & $\emptyset$ & $\emptyset$ \\
& 1 & 0.976 & -0.014 & $5.15 \mathrm{e}-02$ & 3860 \\
& 5 & 0.937 & -0.011 & $1.30 \mathrm{e}-01$ & 17216 \\
\hline
\end{tabular}

Tabela 5.6: Rotação do cone com reconstrução de $2^{a}$ ordem.

\begin{tabular}{|c|c|c|c|c|c|}
\hline $\begin{array}{c}\text { Número de } \\
\text { vértices }\end{array}$ & $\begin{array}{c}\text { Número de } \\
\text { revoluções }\end{array}$ & $\begin{array}{c}\phi_{a} \\
\text { máx. }\end{array}$ & $\begin{array}{c}\phi_{a} \\
\text { min. }\end{array}$ & $L_{\infty}$ & $\begin{array}{c}\text { Tempo } \\
(\mathrm{s})\end{array}$ \\
\hline \hline \multirow{3}{*}{4545} & inicial & 1.0 & 0.0 & $\emptyset$ & $\emptyset$ \\
& 1 & 0.733 & -0.017 & $2.51 \mathrm{e}-01$ & 460 \\
& 5 & 0.473 & -0.017 & $5.32 \mathrm{e}-01$ & 2194 \\
\hline \multirow{3}{*}{17890} & inicial & 1,0 & 0,0 & $\emptyset$ & $\emptyset$ \\
& 1 & 0.955 & -0.028 & $1.08 \mathrm{e}-01$ & 1873 \\
71119 & 5 & 0.833 & -0.085 & $3.23 \mathrm{e}-01$ & 9593 \\
\hline \multirow{3}{*}{} & 1 & 0.975 & 0.001 & $2.90 \mathrm{e}-02$ & 9420 \\
& 5 & 0.957 & -0.005 & $4.48 \mathrm{e}-02$ & 36858 \\
\hline
\end{tabular}

Tabela 5.7: Rotação do cone com reconstrução de $3^{a}$ ordem. 


\begin{tabular}{|c|c|c|c|c|c|}
\hline $\begin{array}{c}\text { Número de } \\
\text { vértices }\end{array}$ & $\begin{array}{c}\text { Número de } \\
\text { revoluções }\end{array}$ & $\begin{array}{c}\phi_{a} \\
\text { máx. }\end{array}$ & $\begin{array}{c}\phi_{a} \\
\text { min. }\end{array}$ & $L_{\infty}$ & $\begin{array}{c}\text { Tempo } \\
(\mathrm{s})\end{array}$ \\
\hline \hline \multirow{3}{*}{4545} & inicial & 1.0 & 0.0 & $\emptyset$ & $\emptyset$ \\
& 1 & 0.946 & -0.022 & $7.24 \mathrm{e}-02$ & 1765 \\
& 5 & 0.812 & -0.052 & $2.05 \mathrm{e}-01$ & 8808 \\
\hline \multirow{3}{*}{17890} & inicial & 1.0 & 0.0 & $\emptyset$ & $\emptyset$ \\
& 1 & 0.979 & -0.015 & $3.04 \mathrm{e}-02$ & 9737 \\
71119 & 5 & 0.934 & -0.085 & $4.93 \mathrm{e} 0-02$ & 35357 \\
\hline \multirow{3}{*}{} & inicial & 1.0 & 0.0 & $\emptyset$ & $\emptyset$ \\
& 1 & 0.983 & 0.001 & $2.66 \mathrm{e}-02$ & 29881 \\
& 5 & 0.952 & -0.003 & $4.23 \mathrm{e}-02$ & 348047 \\
\hline
\end{tabular}

Tabela 5.8: Rotação do cone com reconstrução de $4^{a}$. ordem.

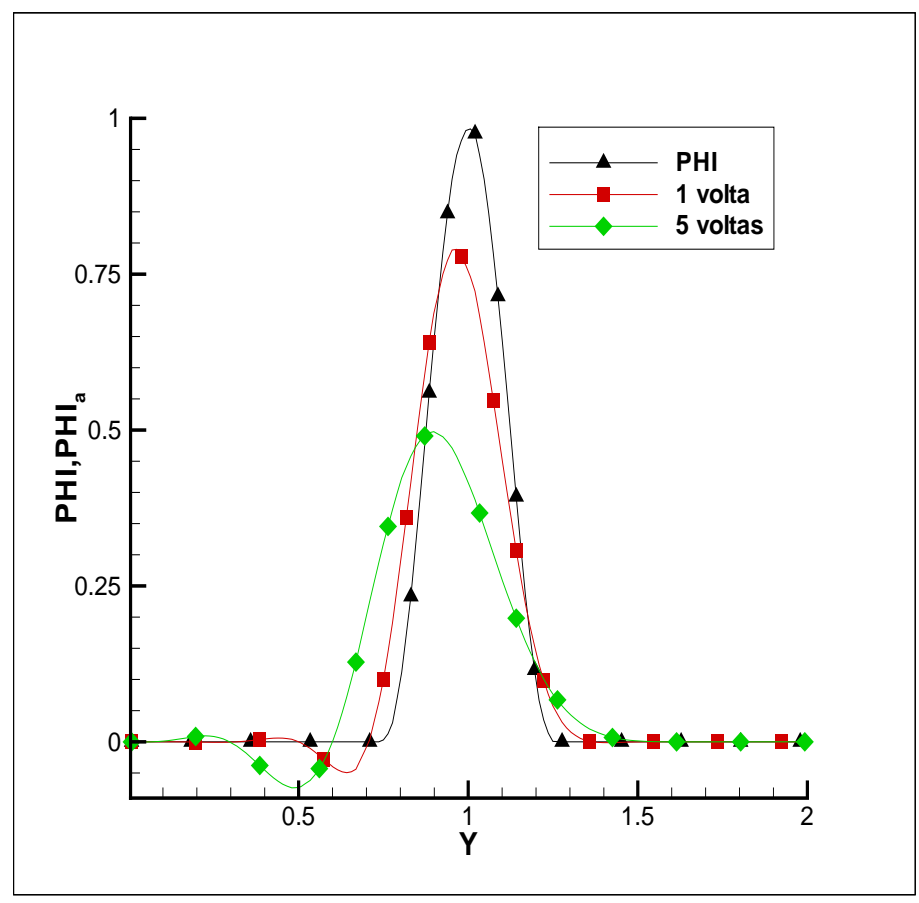

(a) 


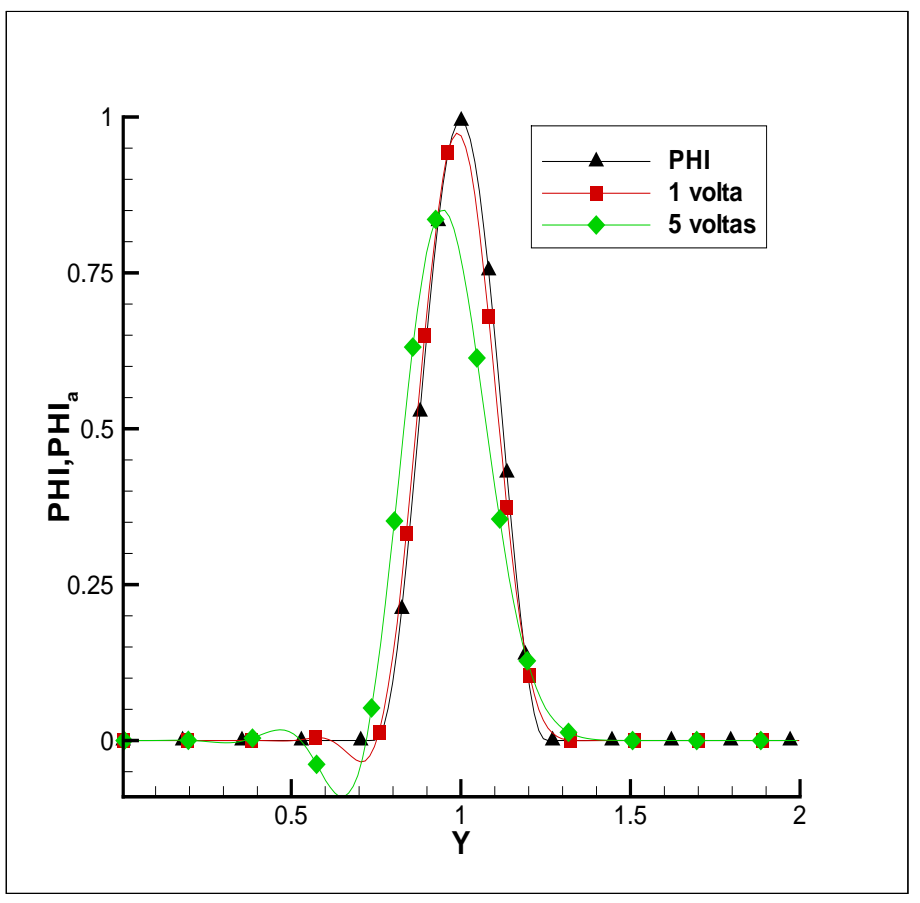

(b)

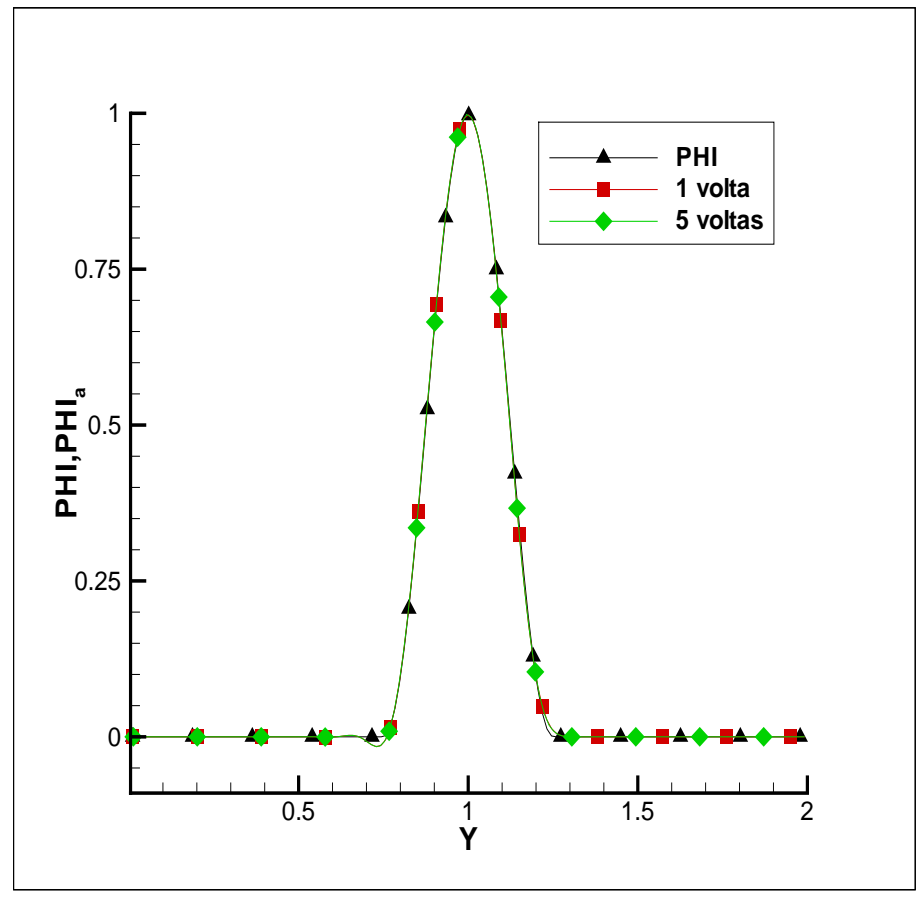

(c)

Figura 5.17: Corte de perfil no cone de revolução para $x=0.5$, na comparação após uma rotação e 5 rotações, reconstrução da solução de $2^{a}$ ordem: (a) malha 60x60 (4545 vértices); (b) malha 120x120 (17890 vértices); (c) malha 240x240 (71119 vértices). 


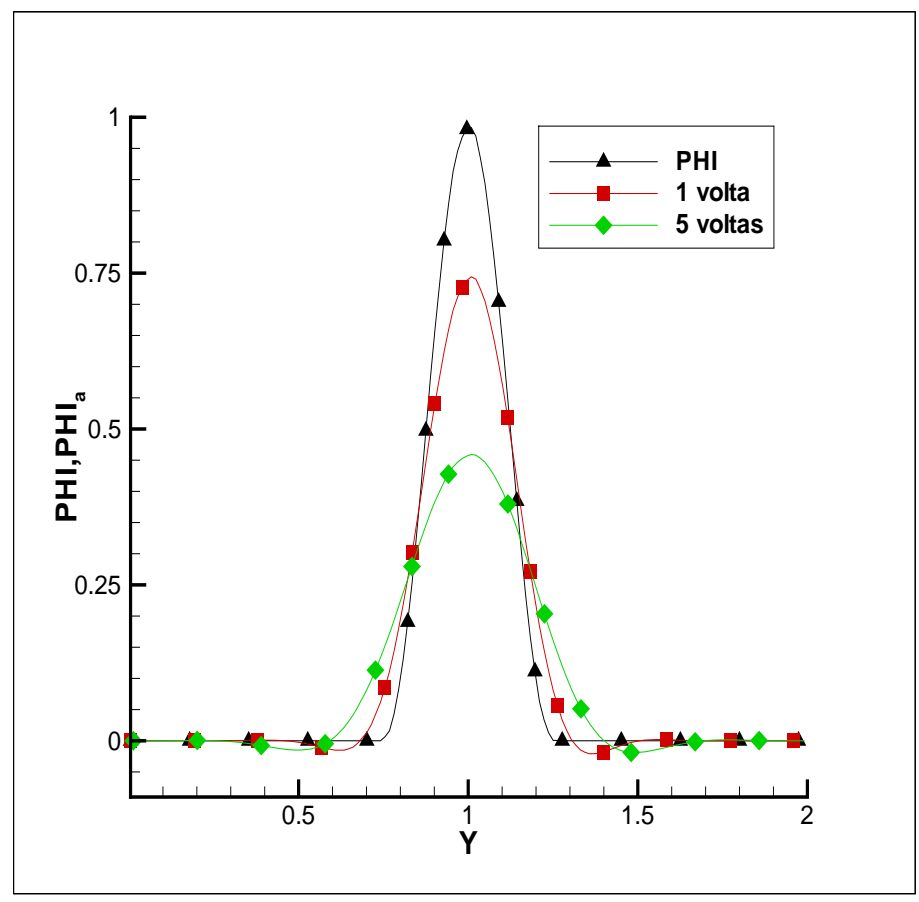

(a)

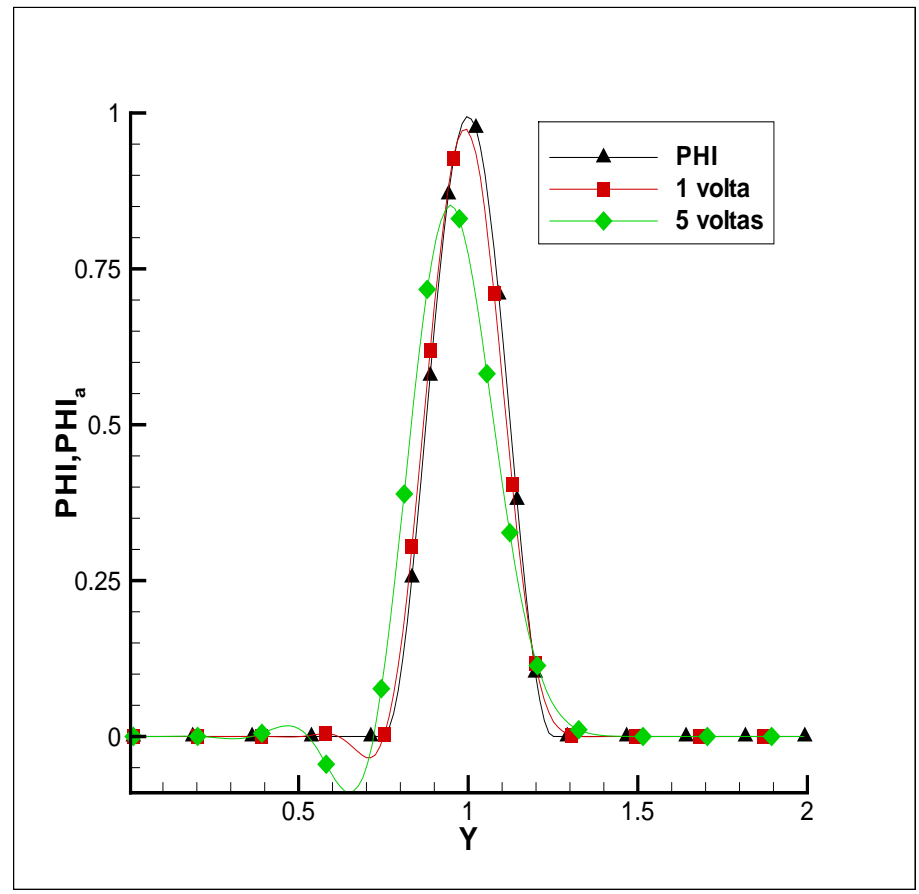

(b) 


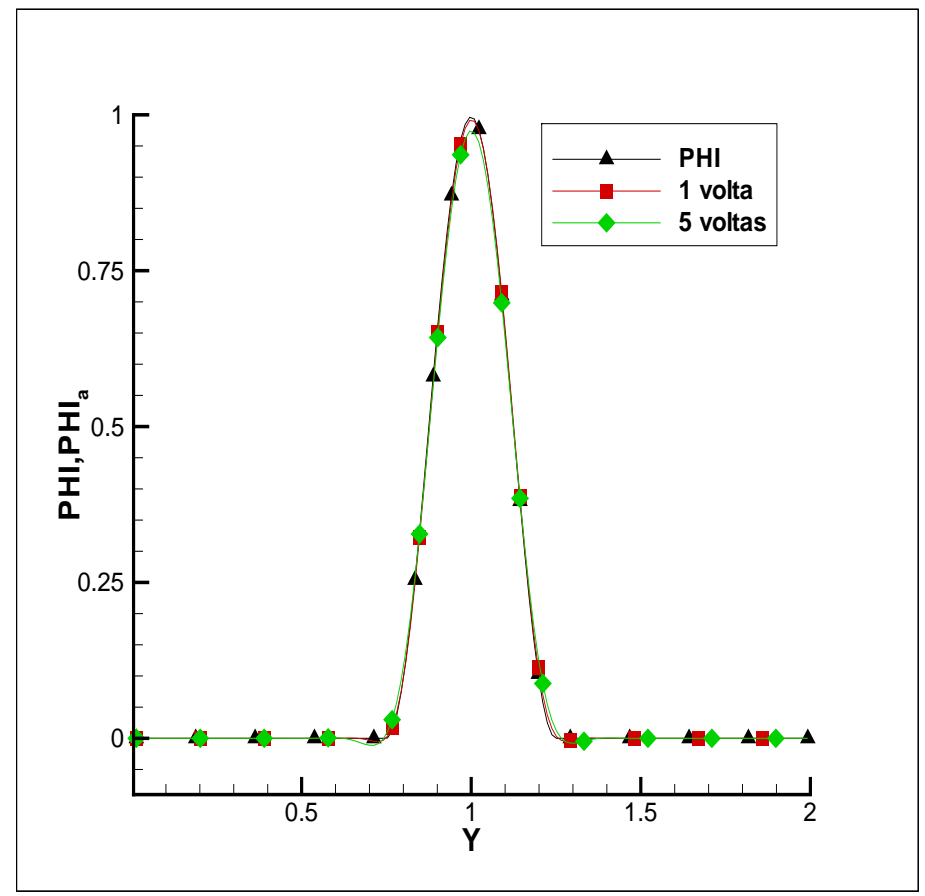

(c)

Figura 5.18: Corte de perfil no cone de revolução para $x=0.5$, na comparação após uma rotação e 5 rotações, reconstrução da solução de $3^{a}$ ordem: (a) malha 60x60 (4545 vértices); (b) malha 120x120 (17890 vértices); (c) malha 240x240 (71119 vértices). 


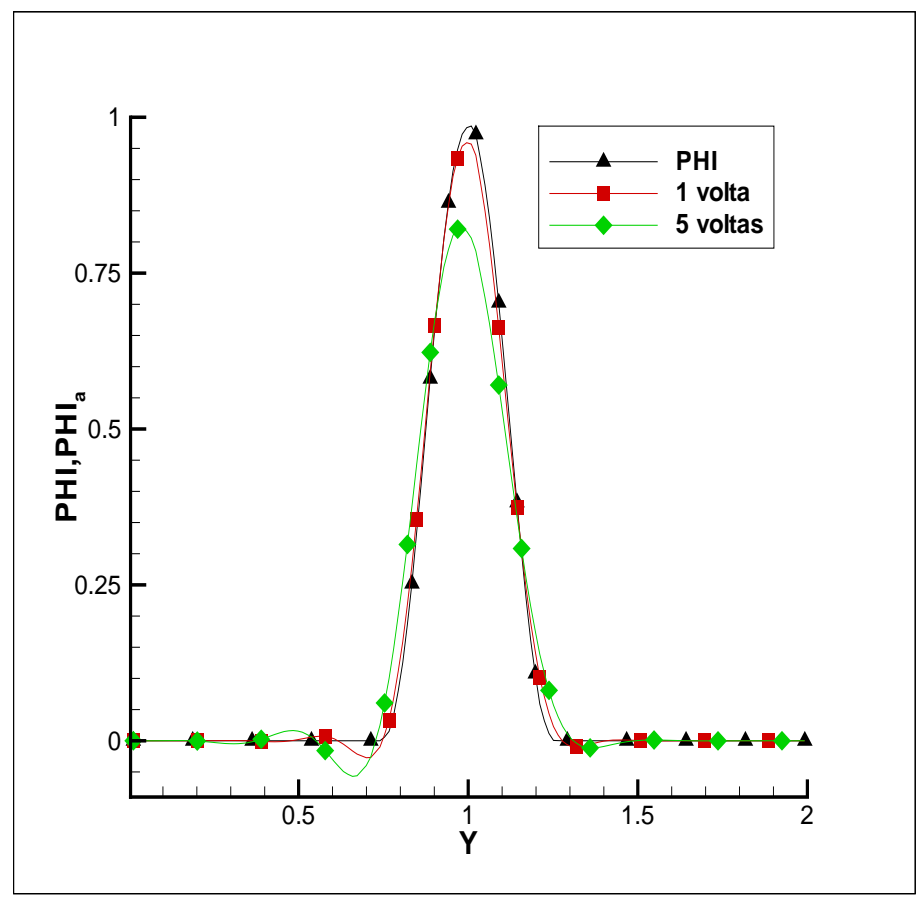

(a)

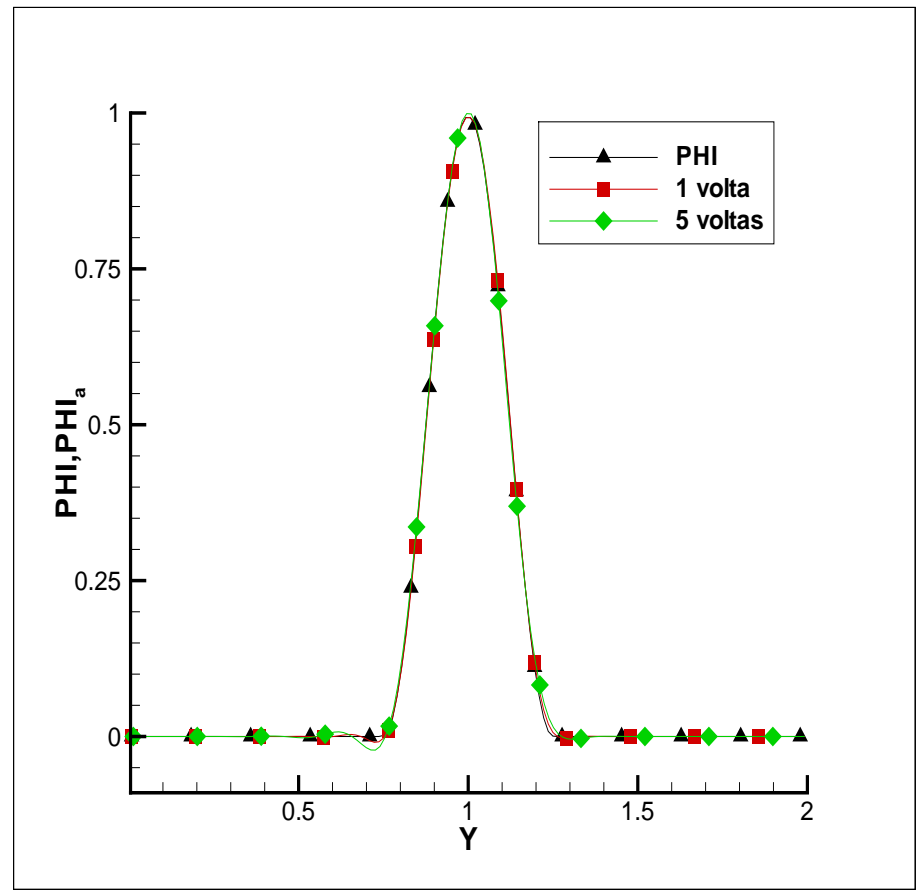

(b) 


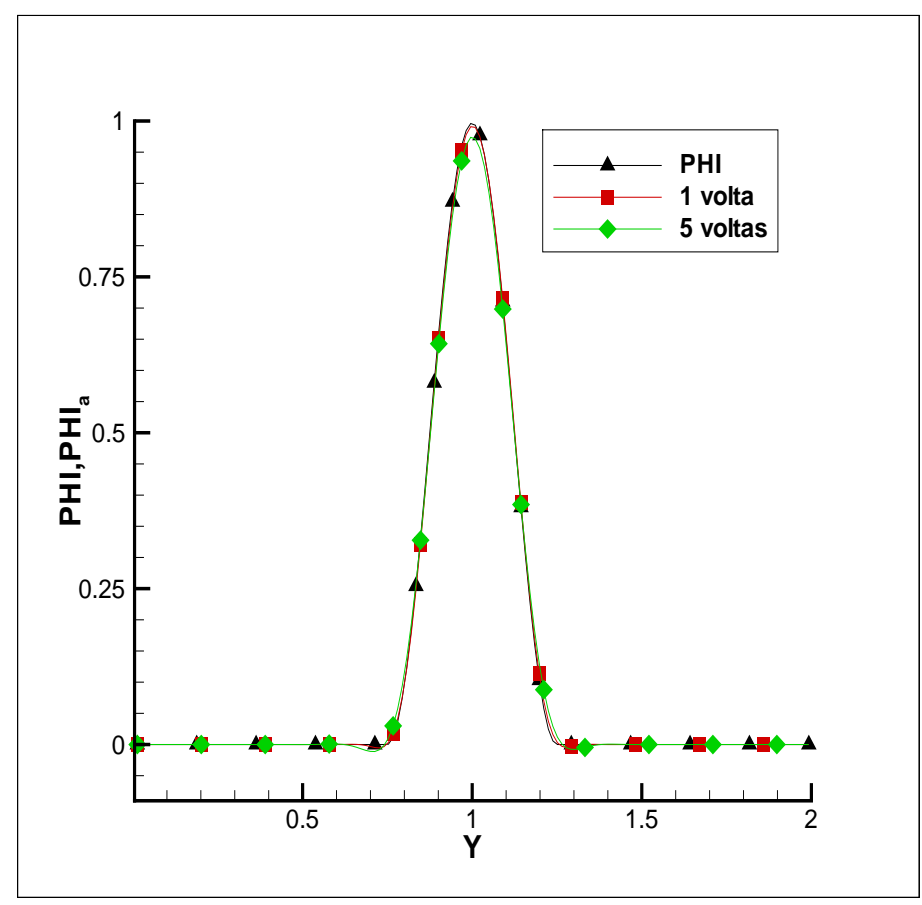

(c)

Figura 5.19: Corte de perfil no cone de revolução para $x=0.5$, na comparação após uma rotação e 5 rotações, reconstrução da solução de $4^{a}$. ordem: (a) malha 60x60 (4545 vértices); (b) malha 120x120 (17890 vértices); (c) malha 240x240 (71119 vértices).

\subsection{Função de Harten}

Para fazer uma análise do comportamento do método de alta ordem com reconstrução mínimos quadrados em descontinuidades, fez-se um teste com a função de Harten (5.10). A forma de escrevê-la e apresentá-la tem sido variada, como por exemplo em [3, 25, 26], mas pelo que consta em Abgrall [1], esta função é conhecida como Função de Harten.

A forma adotada neste trabalho é apresentada por [25], no quadrado $\Omega=[-1,1] \times$ $[-1,1]$. Os testes foram feitos nas malhas da Tabela 5.9 .

$$
\phi(x, y)= \begin{cases}f\left(x-\cot \left(\sqrt{\frac{\pi}{2}}\right) y\right. & , x \leq \frac{\cos (\pi y)}{2} \\ f\left(x+\cot \left(\sqrt{\frac{\pi}{2}}\right) y+\cos (2 \pi y)\right. & , x>\frac{\cos (\pi y)}{2}\end{cases}
$$


com

$$
f(r)= \begin{cases}-r \sin \left(\frac{3 \pi}{2} r^{2}\right) & , r \leq-\frac{1}{3} \\ |\sin (2 \pi r)| & ,|r|<\frac{1}{3} \\ 2 r-1+\frac{1}{6} \sin (3 \pi r) & , r \geq \frac{1}{3}\end{cases}
$$

\begin{tabular}{|c|c|c|c|c|}
\hline Malha & $\mathrm{n} \times \mathrm{n}$ & Células & Vértices & Faces \\
\hline H1 & $20 \times 20$ & 988 & 535 & 1522 \\
\hline H2 & $50 \times 50$ & 6144 & 3173 & 9316 \\
\hline H3 & $150 \times 150$ & 55300 & 27951 & 83250 \\
\hline
\end{tabular}

Tabela 5.9: Malhas utilizadas para o teste com a função de Harten: $n$ é o número de partições em cada lado do domínio $\Omega=[-1,1] \times[-1,1]$.

Esta função é composta de porções suaves separadas por descontinuidades e extremidades, como pode ser visto na Figura 5.20 (a) - (d), para uma reconstrução linear.

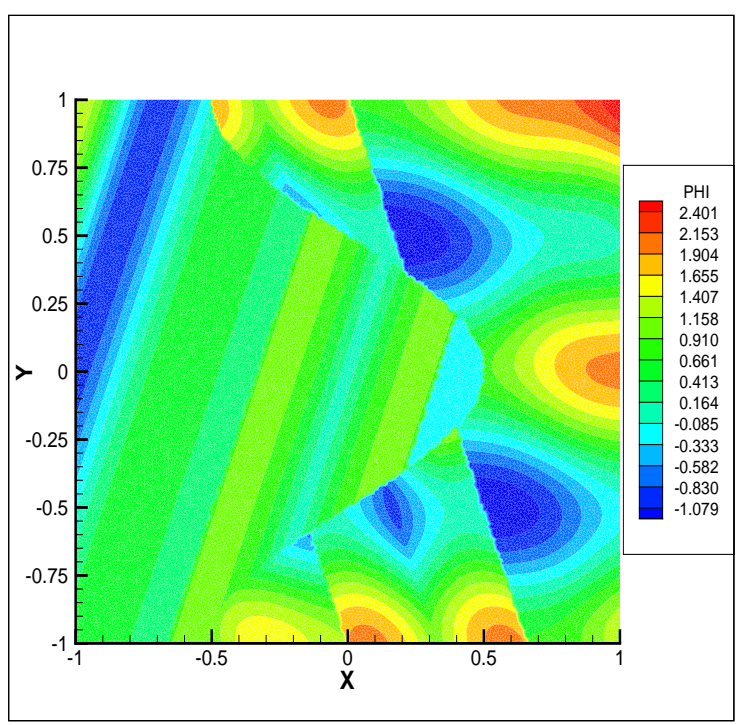

(a)

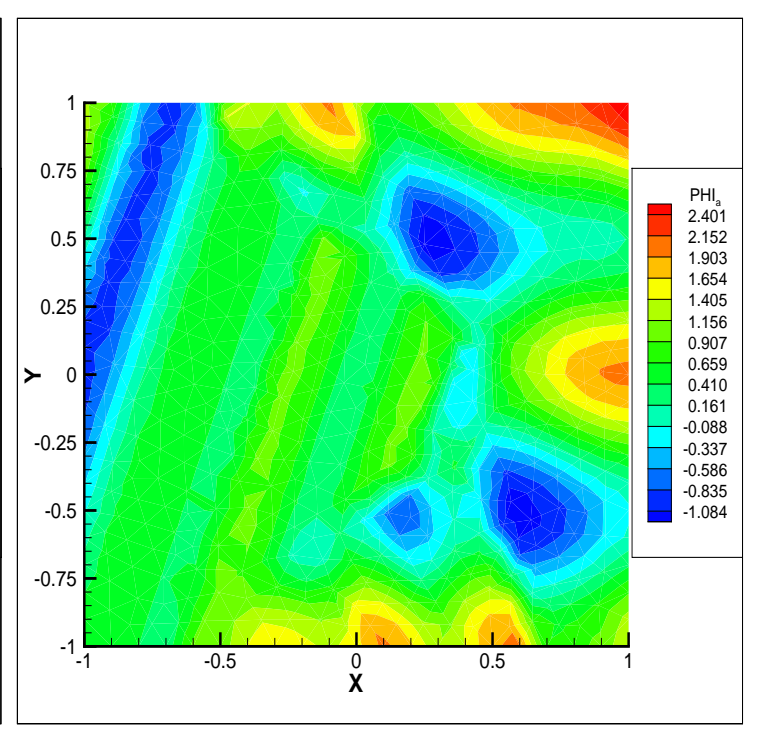

(b) 


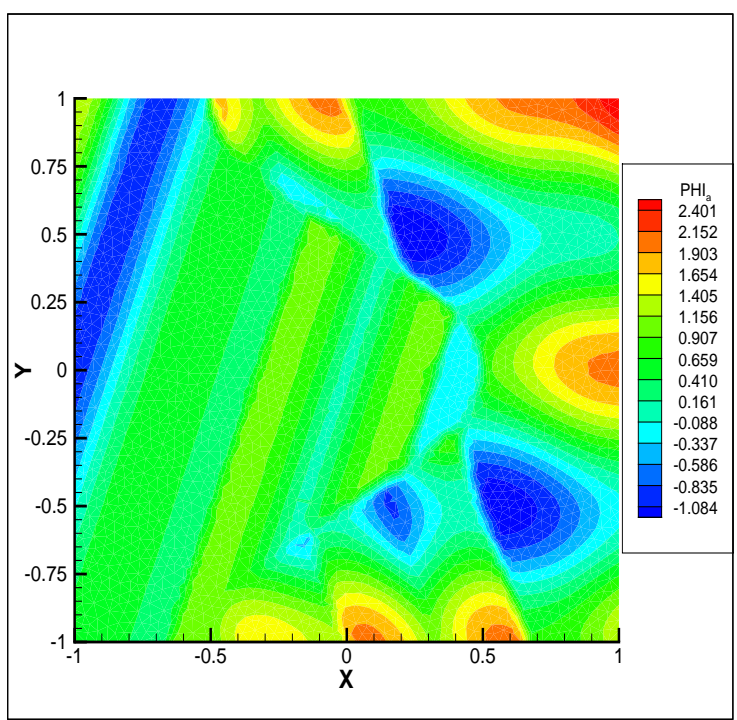

(c)

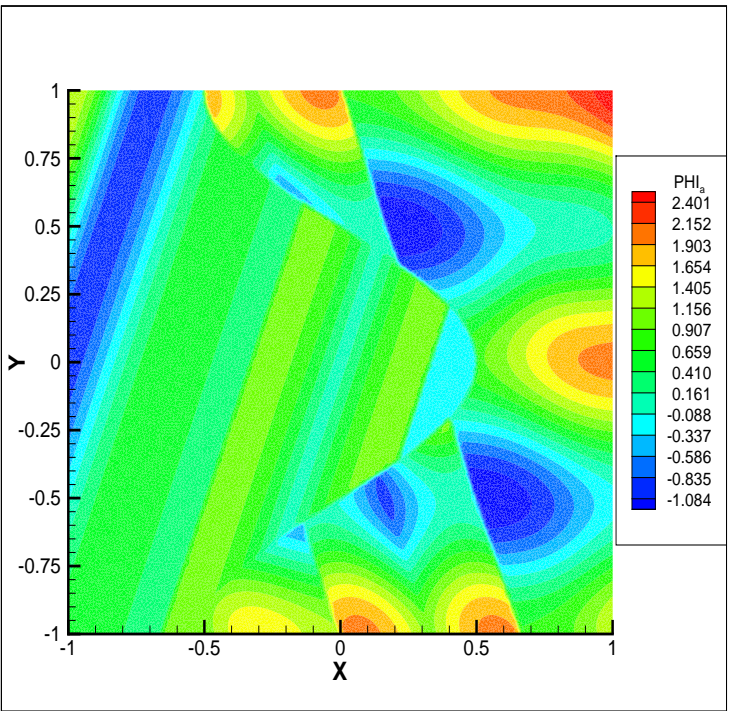

(d)

Figura 5.20: Reconstrução linear da função de Harten: (a) função de Harten exata; (b) malha H1 (535 vértices); (c) malha H2 (3173 vértices); (d) malha H3 (27951 vértices).

Um corte de perfil na horizontal $(y=0)$, Figura 5.21, e no corte na vertical $(x=0)$ foi feito e vê-se o comportamento da reconstrução nas descontinuidades, se aproximando do perfil exato e suavizando a descontinuidade à medida que se refina a malha.

Com base nos testes efetuados, pode-se dizer que a técnica é apropriada ao problema de interesse. 


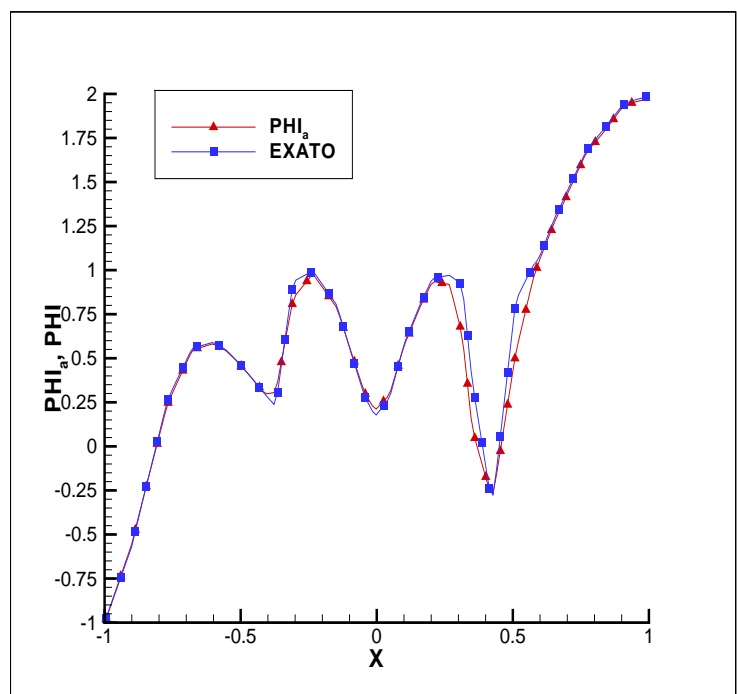

(a)

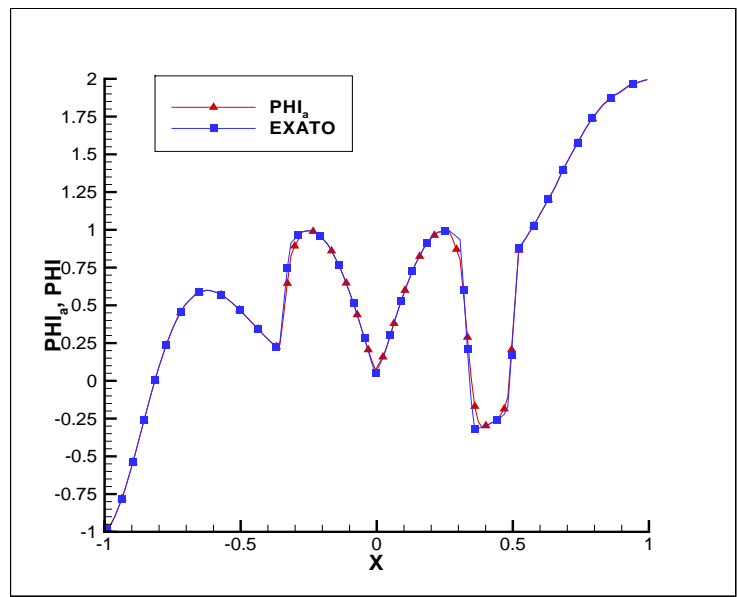

(b)

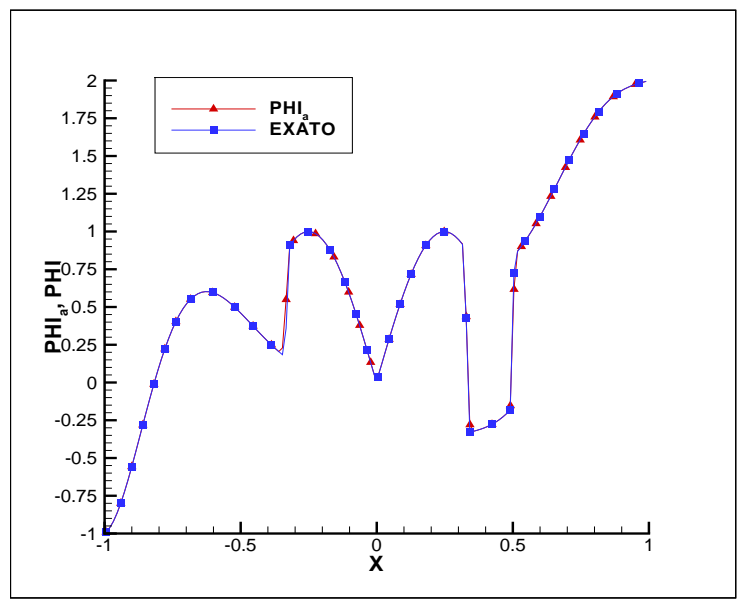

(c)

Figura 5.21: Corte horizontal em $y=0.0$ na reconstrução linear da Função de Harten: (a) malha H1 (535 vértices); (b) malha H2 (3173 vértices); (c) malha H3 (27951 vértices). 


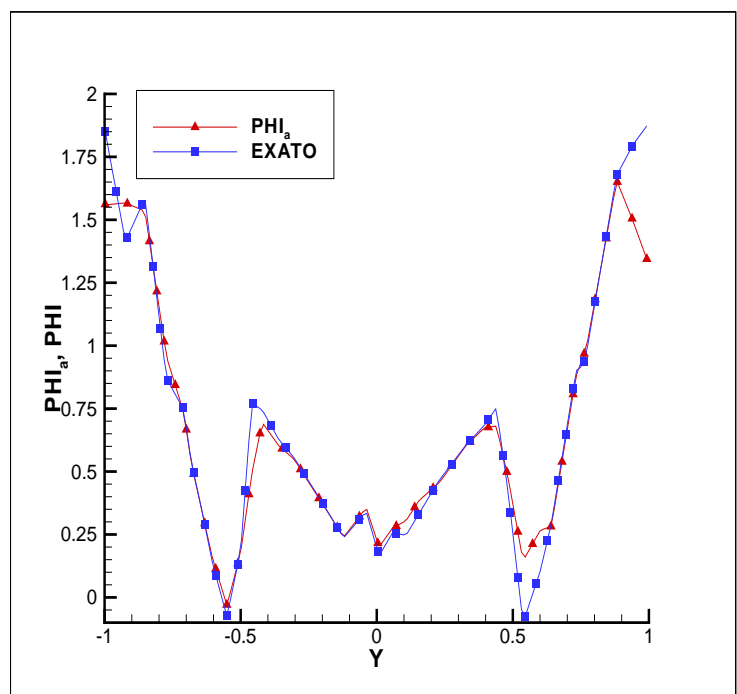

(a)

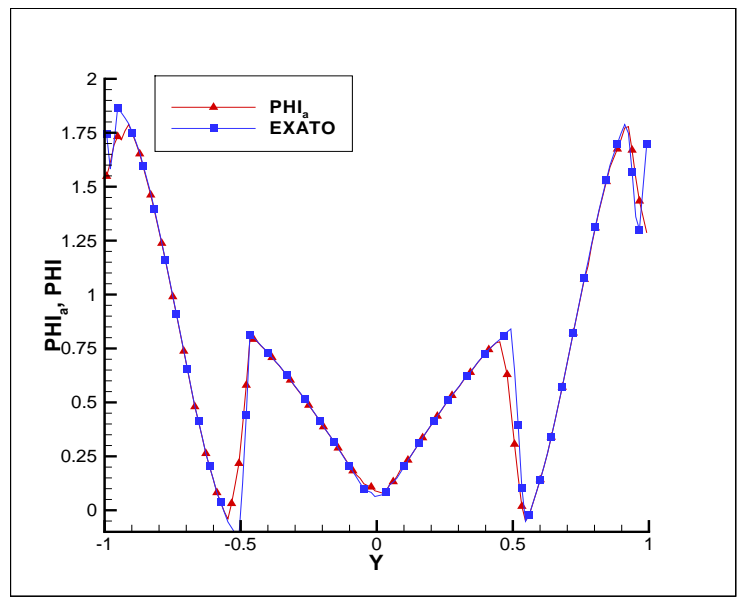

(b)

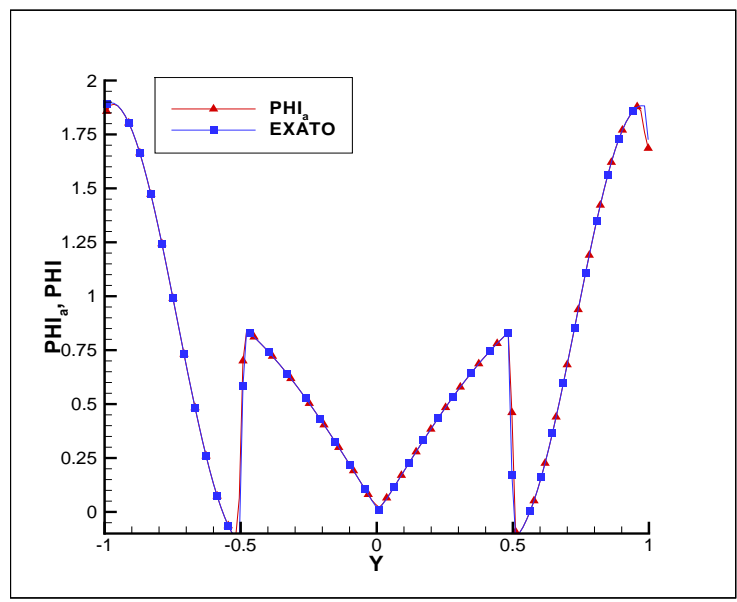

(c)

Figura 5.22: Corte vertical em $x=0.0$ na reconstrução linear da função de Harten: (a) malha H1 (535 vértices); (b) malha H2 (3173 vértices); (c) malha H3 (27951 vértices). 
Capítulo 6

\section{Aplicação no transporte euleriano de gotículas}

Neste capítulo será apresentada uma aplicação da reconstrução mínimos quadrados com alta ordem na equação de advecção para o transporte de uma propriedade $\phi$. Trata-se de aplicar a equação de advecção para uma variável $\phi$, com campo de velocidade obtido através do solver NSC2KE, seção 3.3, e também para o campo de velocidade constante $\mathrm{V}=(1,0)$.

Nas simulações numéricas, os tempos de execução considerados são das máquinas do LCCA, Apêndice C. Os testes foram feitos para cada ordem de reconstrução e o critério de parada é dado pelo número máximo de iterações (5000 iterações) ou quando o resíduo atinge uma tolerância de $1.0 \times 10^{-7}$, com $C F L=0.4$.

\subsection{Teste 1 - Condição de contorno de Neumann no cilindro}

As malhas usadas neste e nos próximos testes são da Tabela 3.3, seção 3.3.1. O valor da propriedade $\phi$ é 1.0 na entrada do domínio, $x=-2 D$, com condição de contorno de Neumann para os outros três contornos do domínio e igual a zero nos demais pontos da malha.

Neste teste, considerou-se a condição de contorno de Neumann no cilindro, $\frac{\partial \phi}{\partial n}=0.0$, 
para a análise do transporte da propriedade $\phi$ com a variação do número de Reynolds. A Tabela 6.1 apresenta os tempos de execução e o respectivo número de iterações obtidos para a malha $m 5$. Os resultados podem ser vistos nas Figuras 6.1 e 6.4 para $R e=1.5 \times 10^{5}$ e para $R e=6.7 \times 10^{5}$, respectivamente. E nas Figuras 6.2 e 6.5, os vetores do campo de velocidade na região de sombra.

\begin{tabular}{|c|c|c|c|c|c|}
\hline ordem & malha & no. de vértices & Re & tempo(s) & iterações \\
\hline 3 & $\mathrm{~m} 5$ & 26866 & $1.5 \times 10^{5}$ & 6881 & 2229 \\
\hline 3 & $\mathrm{~m} 5$ & 26866 & $6.7 \times 10^{5}$ & 6248 & 2239 \\
\hline
\end{tabular}

Tabela 6.1: Tempo de execução para a condição de contorno de Neumann no cilindro empregando a malha m5.

As Figuras 6.3 e 6.6 apresentam a distribuição da propriedade $\phi$ ao longo do cilindro após a convergência. Observa-se que para $R e=6.7 \times 10^{5}$, o ponto de descolamento ocorre mais próximo do vértice $(\mathrm{D}, 0)$, com isto o valor de $\phi$ decai de próximo a 1.0 para zero, voltando para próximo de 1.0 devido à recirculação atrás do cilindro atraindo $\phi$ para o contorno atrás do cilindro, de forma mais acentuada que para $R e=1.5 \times 10^{5}$.

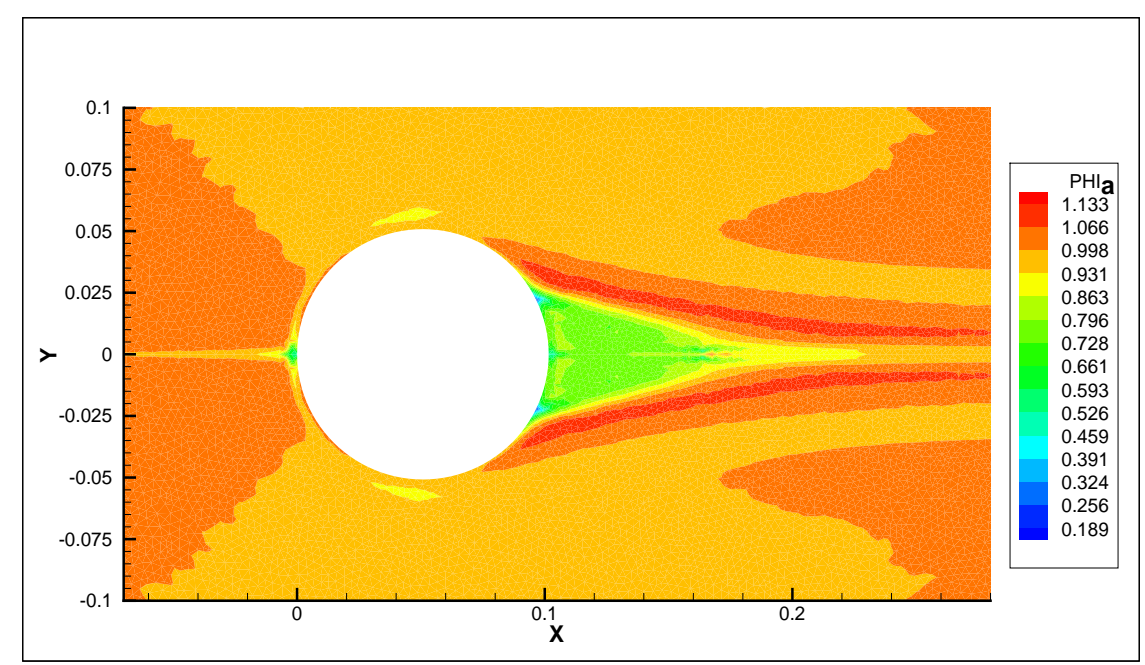

Figura 6.1: Transporte da propriedade $\phi$ : malha $m 5$ - 26866 vértices, campo de velocidade do NSC2KE para $R e=1.5 \times 10^{5}$ e reconstrução quadrática. 


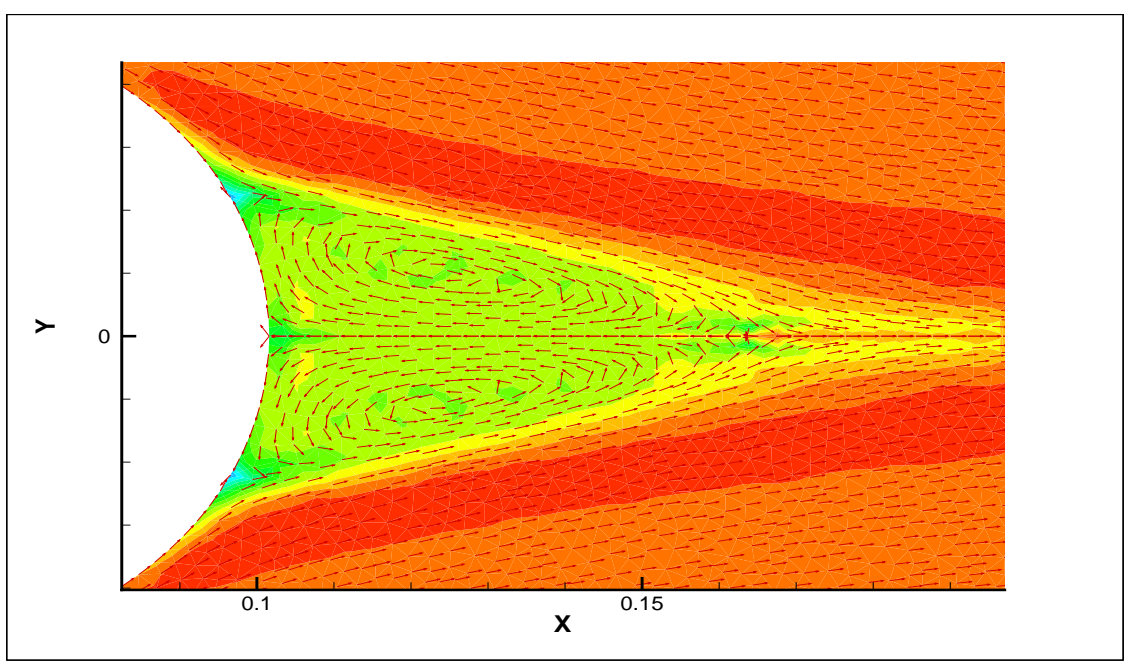

Figura 6.2: Vetores do campo de velocidade na região de sombra na Figura 6.1.

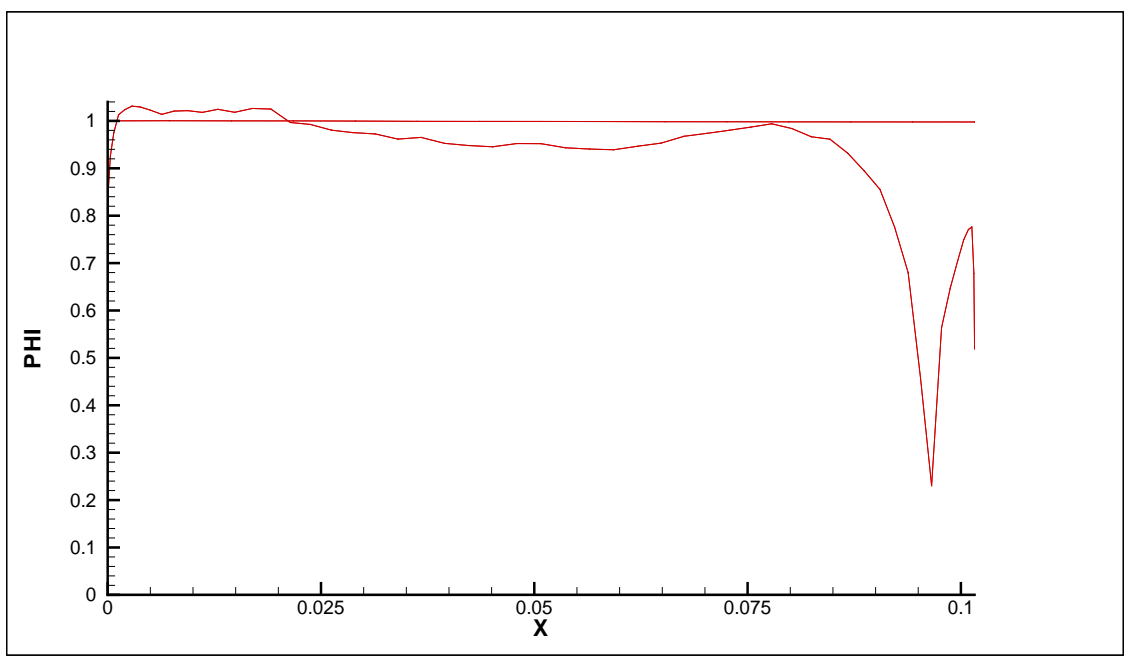

Figura 6.3: Valores da propriedade $\phi$ no cilindro: malha $m 5$ - 26866 vértices, campo de velocidade do NSC2KE para $R e=1.5 \times 10^{5}$ e reconstrução quadrática. 


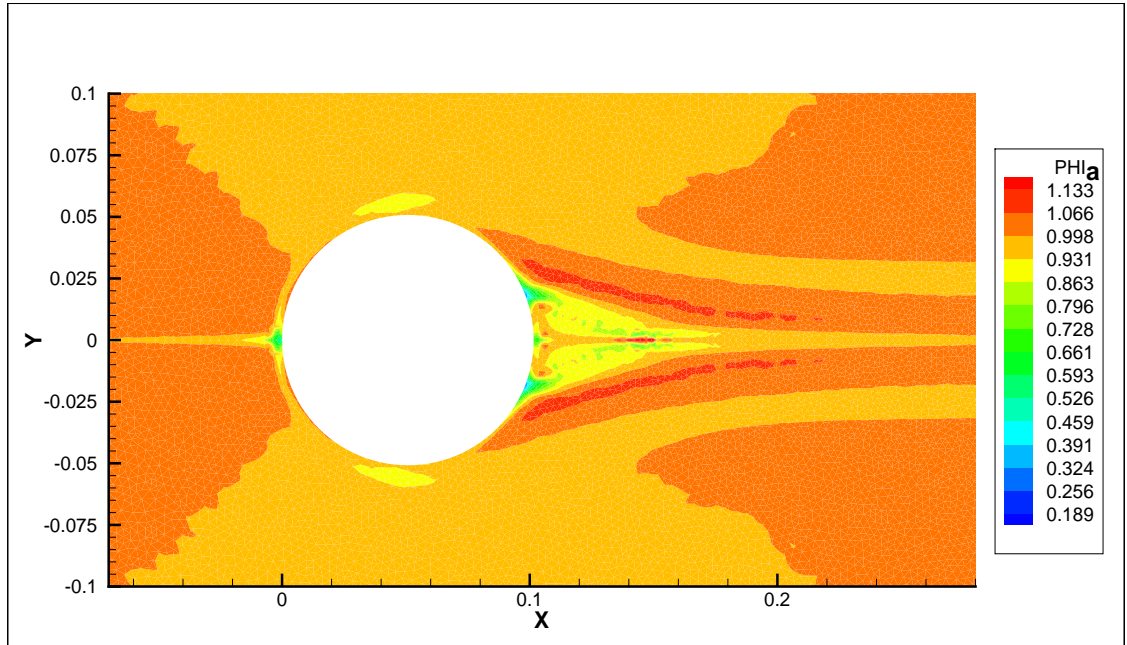

Figura 6.4: Transporte da propriedade $\phi$ : malha $m 5$ - 26866 vértices e campo de velocidade do NSC2KE para $R e=6.7 \times 10^{5}$ e reconstrução quadrática.

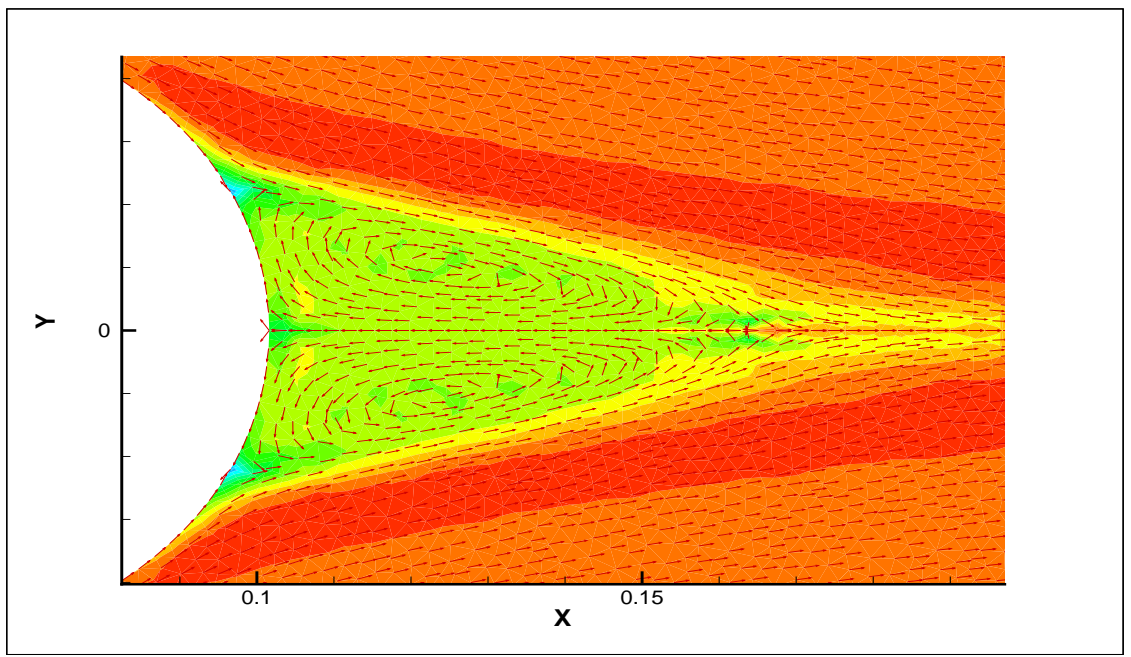

Figura 6.5: Vetores do campo de velocidade na região de sombra na Figura 6.4. 


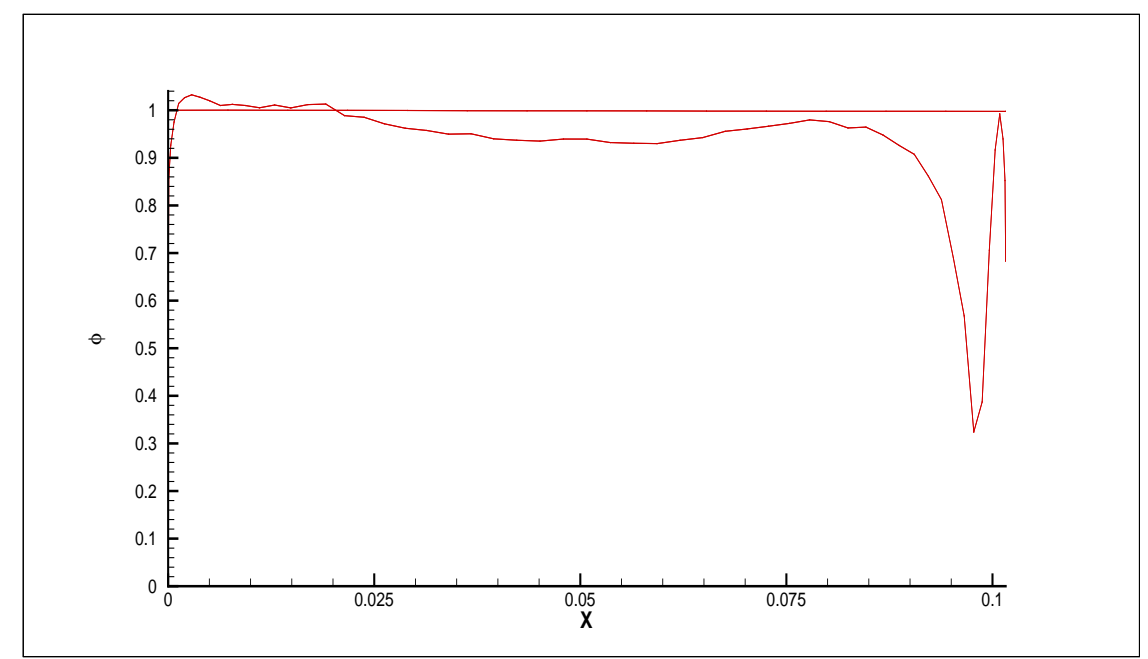

Figura 6.6: Valores da propriedade $\phi$ no cilindro: malha $m 5$ - 26866 vértices e campo de velocidade do NSC2KE para $R e=6.7 \times 10^{5}$ e reconstrução quadrática.

\subsubsection{Teste 2 - Condição de contorno definida pelo vetor veloci- dade}

Neste teste a condição de contorno é definida pelo ponto de descolamento, obtido a partir da mudança de sentido do vetor direção da velocidade sobre o cilindro, como mostra a Figura 6.7, para $R e=1.5 \times 10^{5}$. Do ponto $(0,0)$ até o ponto de descolamento a condição de contorno sobre o cilindro é a condição de Dirichlet, $\phi=0.0$, e do ponto de descolamento até o ponto $(\mathrm{D}, 0)$ a condição estabelecida é a de Neumann, $\frac{\partial \phi}{\partial n}=0.0$.

Na Figura 6.8 tem-se um exemplo deste teste, mostrando o efeito do fenômeno físico da recirculação influenciando, também, a região atrás do cilindro. 


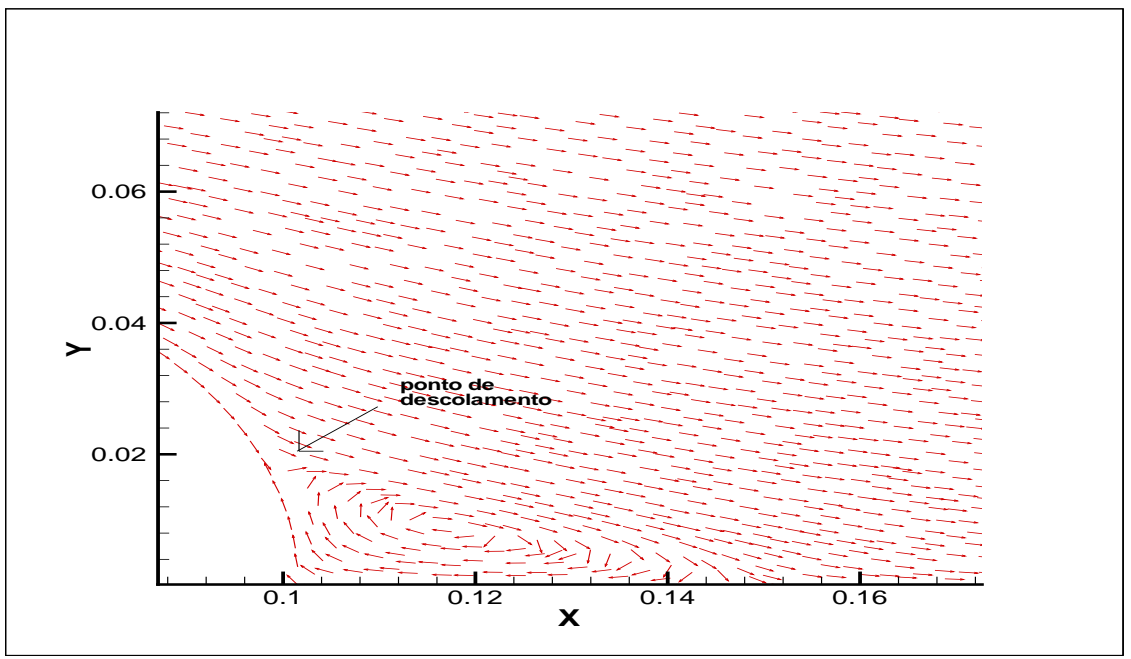

Figura 6.7: Ponto de descolamento definido pelo vetor direção da velocidade.

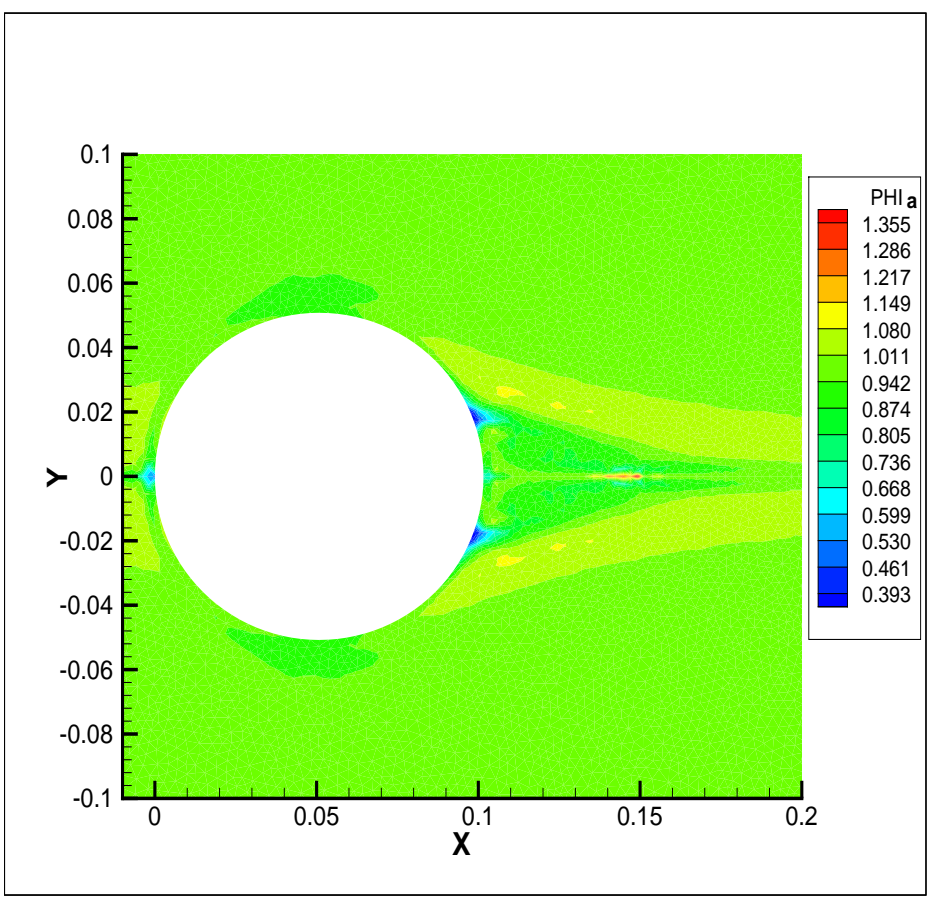

Figura 6.8: Transporte do escalar $\phi$ : malha $m 3$ - 55239 vértices, campo de velocidade do NSC2KE para $R e=1.5 \times 10^{5}$ e reconstrução linear. 


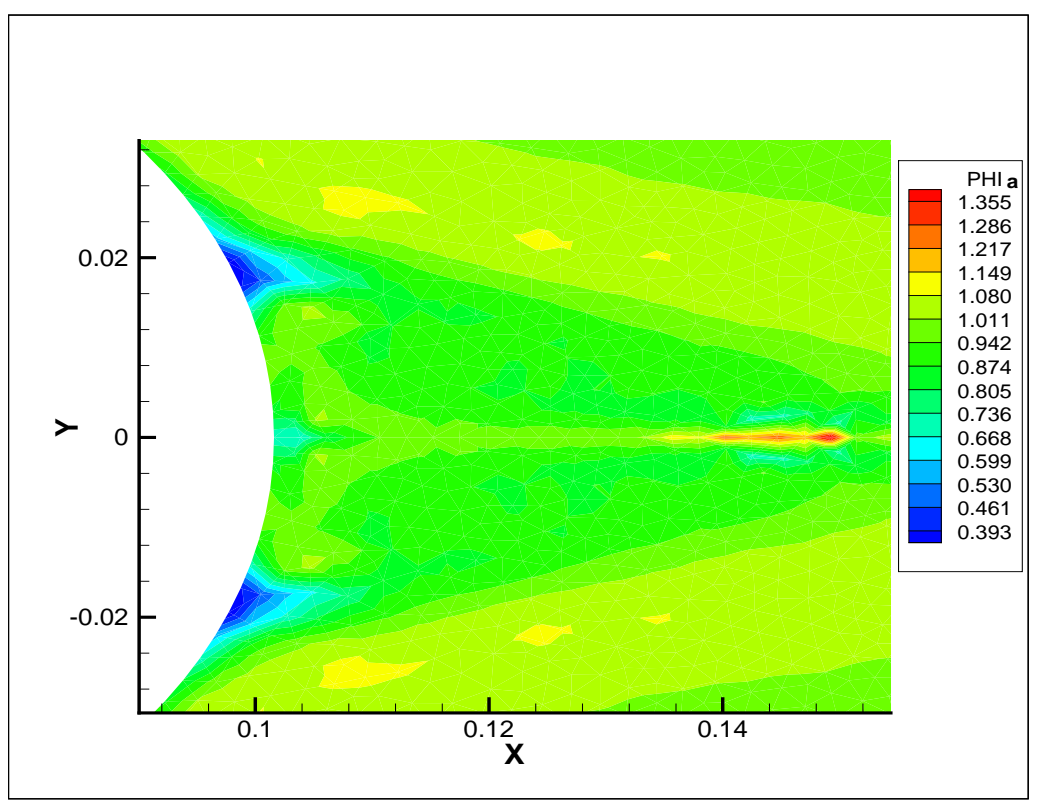

Figura 6.9: Zoom da região de sombra - região atrás do cilindro da Figura 6.8.

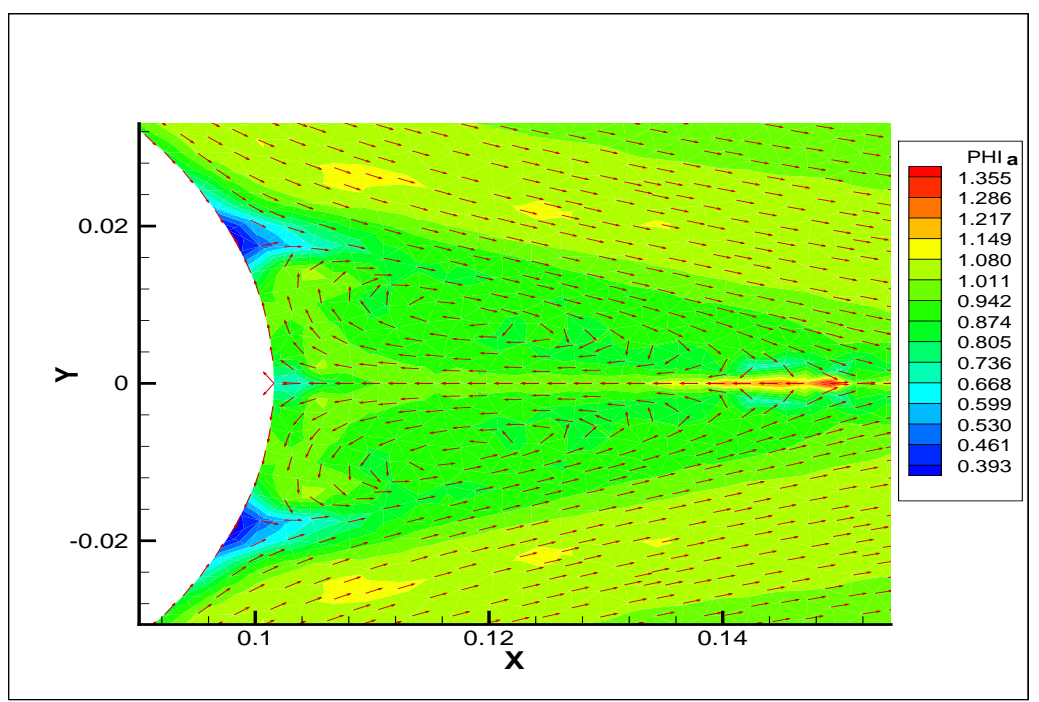

Figura 6.10: Vetores do campo de velocidade na região de sombra na Figura 6.8. 


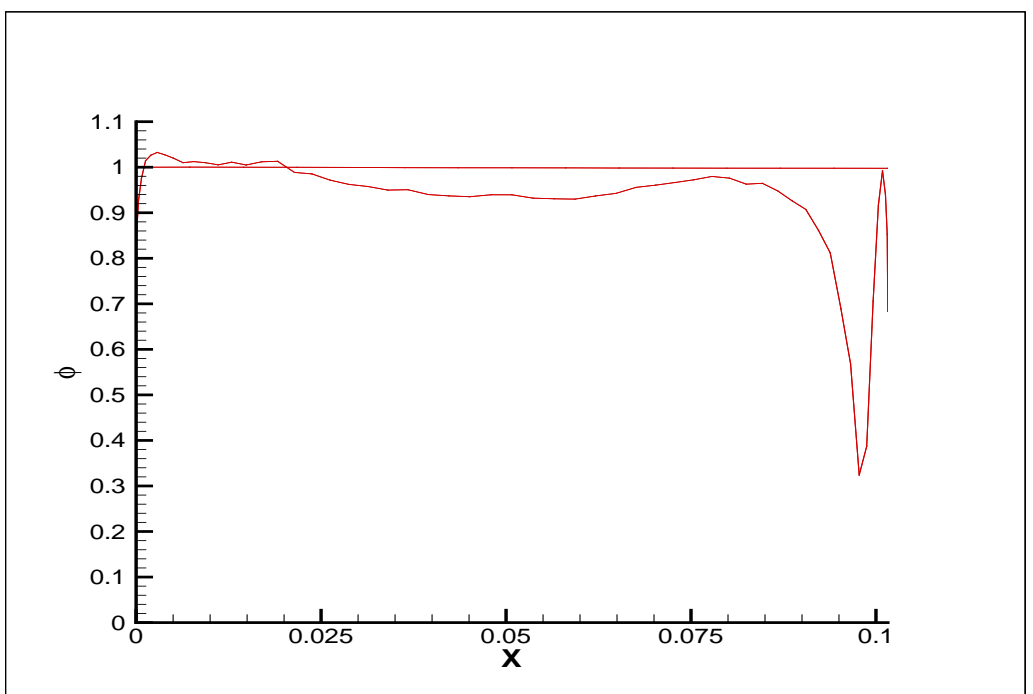

Figura 6.11: Valores da propriedade $\phi$ no cilindro: malha $m 3$ - 55239 vértices, campo de velocidade do NSC2KE para $R e=1.5 \times 10^{5}$ e reconstrução linear.

Este teste buscou mostrar que a recirculação atrás do cilindro influencia nos valores de $\phi$ como observa-se no intervalo $[0.075,0.1]$ da Figura 6.11 que mostra o momento que os valores que $\phi$ começam a receber tal influência. O mesmo foi observado no teste anterior com condição de Neumann, com influência mais significativa para $R e=6.7 \times 10^{5}$. No caso do transporte de gotículas este fenômeno não deve ocorrer, havendo a necessidade de mudar a condição de contorno nesta região. Uma das formas é utilizar a componente da normal $n_{x}$. 


\subsubsection{Teste 3 - Condição de contorno definida pela componente da normal $n_{x}$}

Neste teste a componente da normal $n_{x}$ definirá a condição de contorno sobre o cilindro. Para $n_{x}<0$, o contorno terá condição de Neumann, $\frac{\partial \phi}{\partial n}=0$ e, caso contrário, a condição de contorno será de Dirichlet, $\phi=0$, como mostra a Figura 6.12.

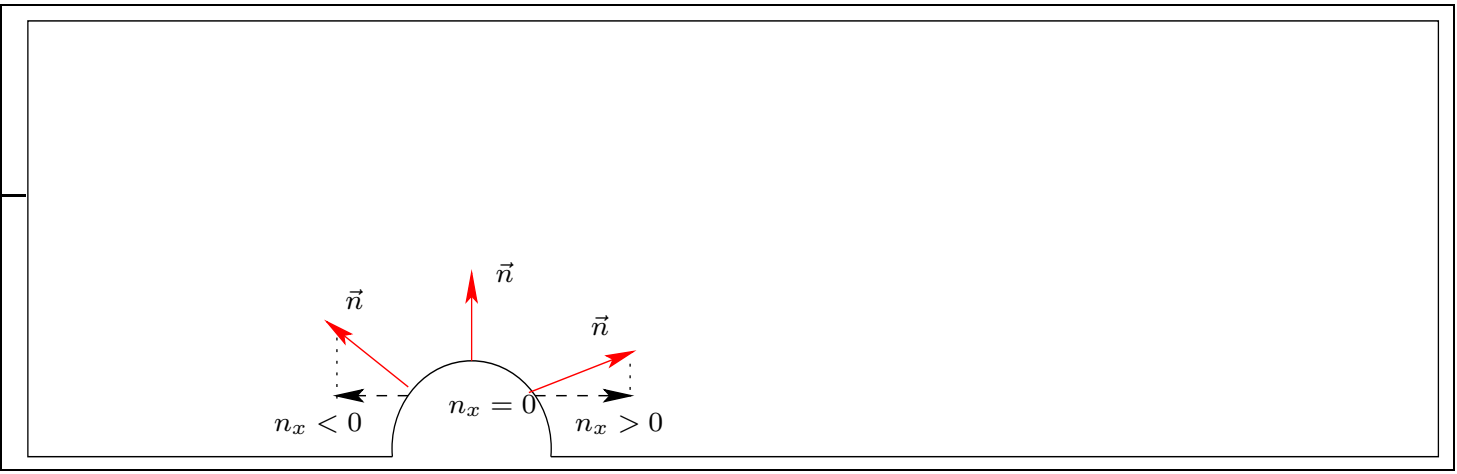

Figura 6.12: Definição das condições de contorno no cilindro através da componente da normal $n_{x}$.

A Tabela 6.2 apresenta os resultados para alguns dos testes realizados. As Figuras 6.13, $6.15,6.17,6.19,6.21$ e 6.23, apresentam os gráficos obtidos na reconstrução da solução e os valores da propriedade $\phi$ no contorno do cilindro nas Figuras 6.14, 6.16, 6.18, 6.20, 6.22 e 6.24. Observa-se, próximo de $\mathrm{x}=0.0$, os valores de $\phi$ apresentando uma certa oscilação provocada por uma pequena recirculação e a partir do ponto $(\mathrm{D} / 2,0)$ tem-se $\phi=0.0$.

\begin{tabular}{|c|c|c|c|c|}
\hline ordem & malha & $\mathrm{n}^{\text {o. }}$ de vértices & tempo $(\mathrm{s})$ & iterações \\
\hline 2 & $\mathrm{~m} 1$ & 3559 & 99 & 742 \\
\hline 3 & $\mathrm{~m} 1$ & 3559 & 241 & 751 \\
\hline 2 & $\mathrm{~m} 2$ & 13664 & 821 & 1438 \\
\hline 2 & $\mathrm{~m} 3$ & 55239 & 7586 & 2795 \\
\hline 2 & $\mathrm{~m} 4$ & 6724 & 329 & 1070 \\
\hline 3 & $\mathrm{~m} 4$ & 6724 & 1135 & 1082 \\
\hline 2 & $\mathrm{~m} 5$ & 26866 & 3116 & 2107 \\
\hline
\end{tabular}

Tabela 6.2: Testes com a componente da normal $n_{x}$ definindo a condição no contorno. 


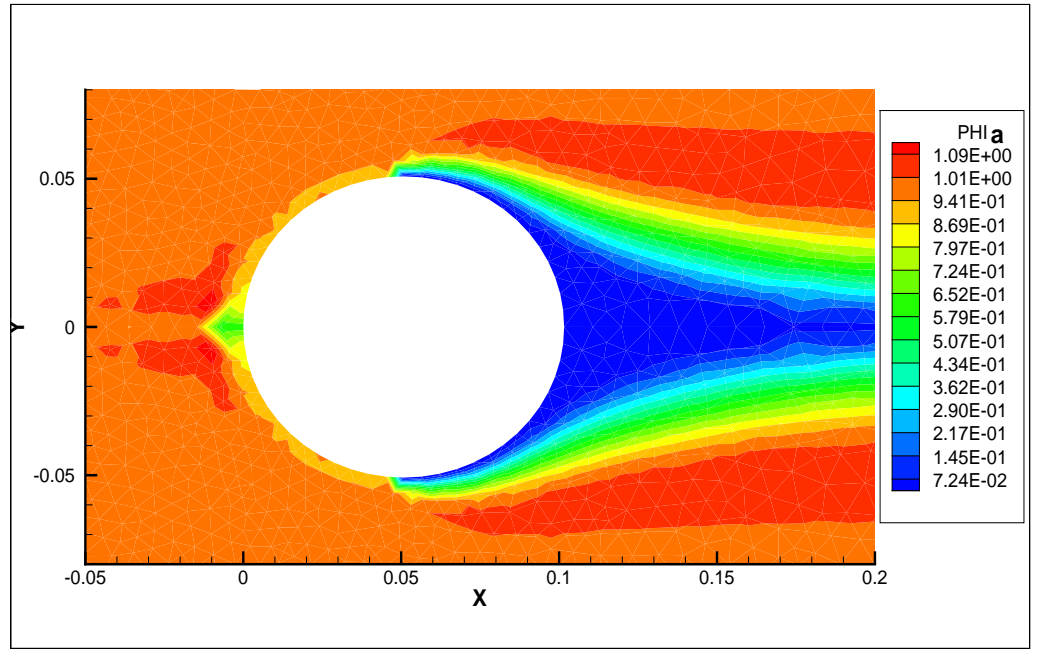

Figura 6.13: Transporte do escalar $\phi$ : malha $m 1$ - 3559 vértices, reconstrução linear.

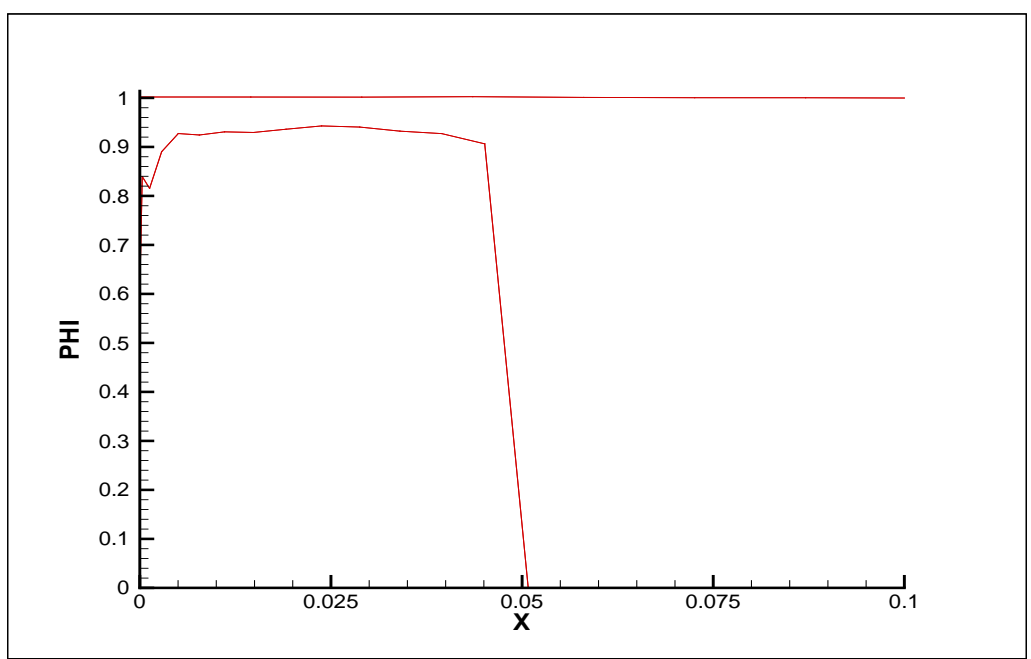

Figura 6.14: Valores da propriedade $\phi$ no cilindro: malha $m 1$ - 3559 vértices, reconstrução linear. 


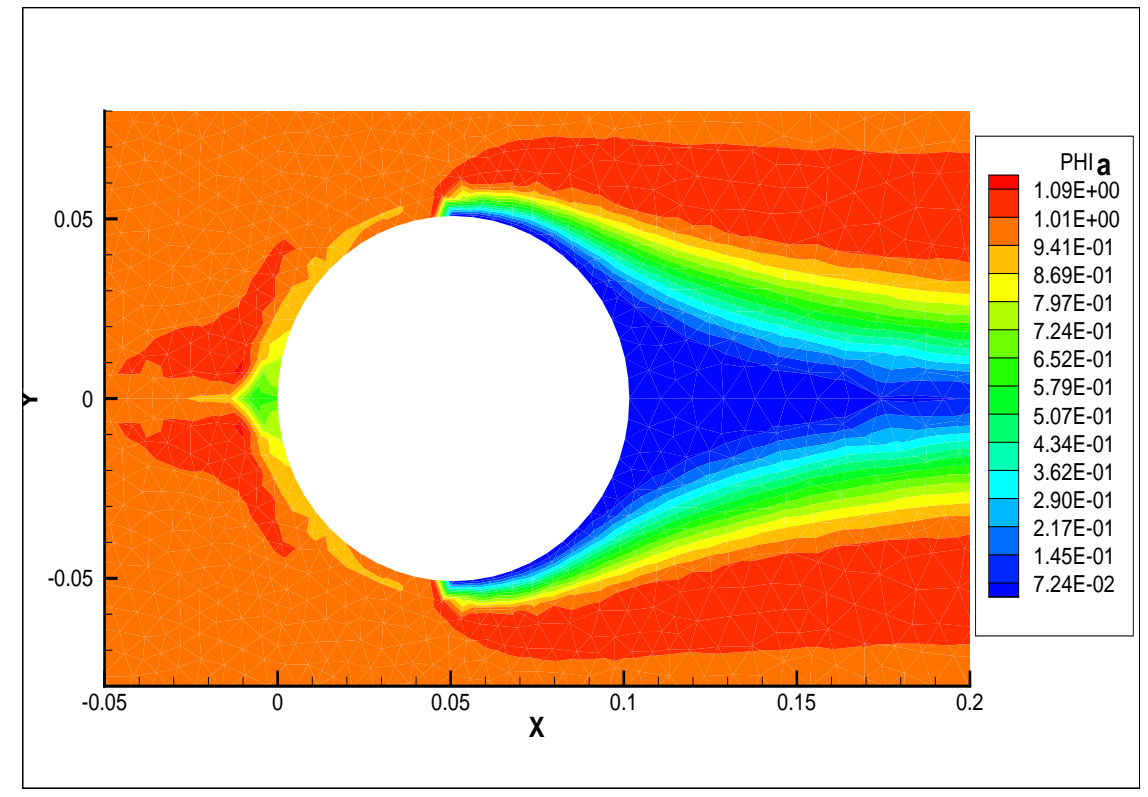

Figura 6.15: Transporte da propriedade $\phi$ : malha $m 1$ - 3559 vértices, reconstrução quadrática.

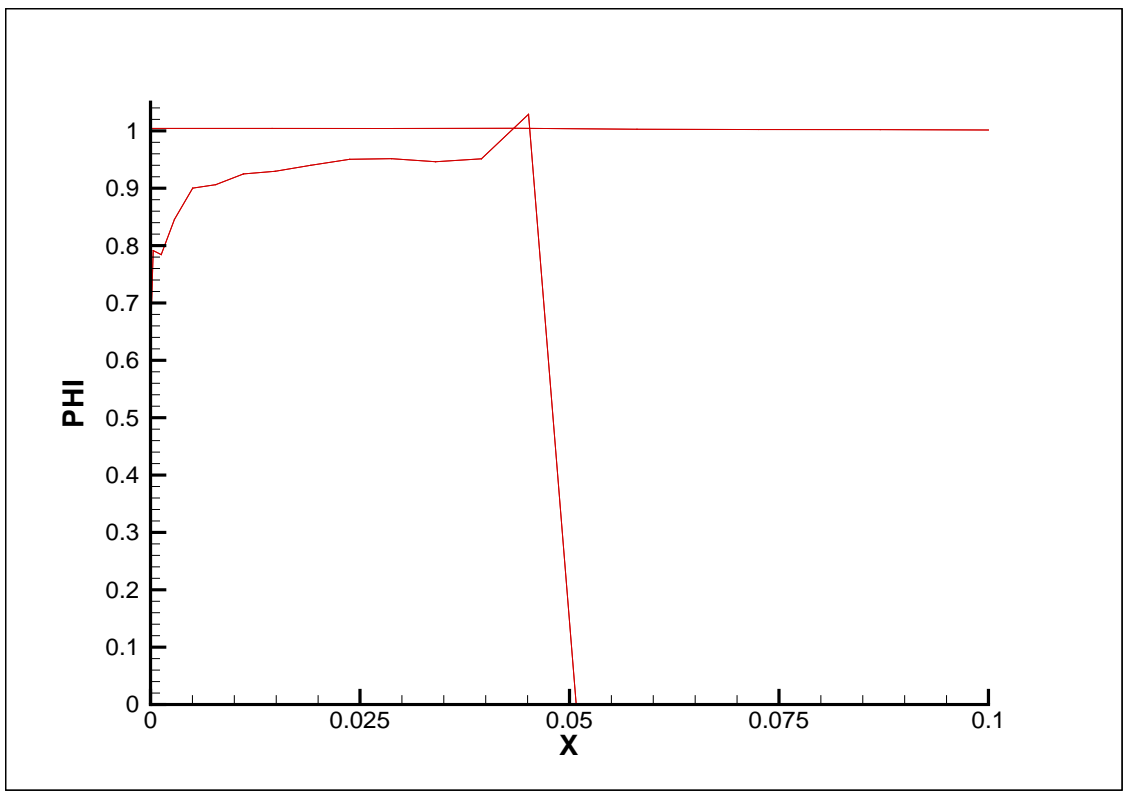

Figura 6.16: Valores da propriedade $\phi$ no cilindro: malha $m 1$ - 3559 vértices, reconstrução quadrática. 


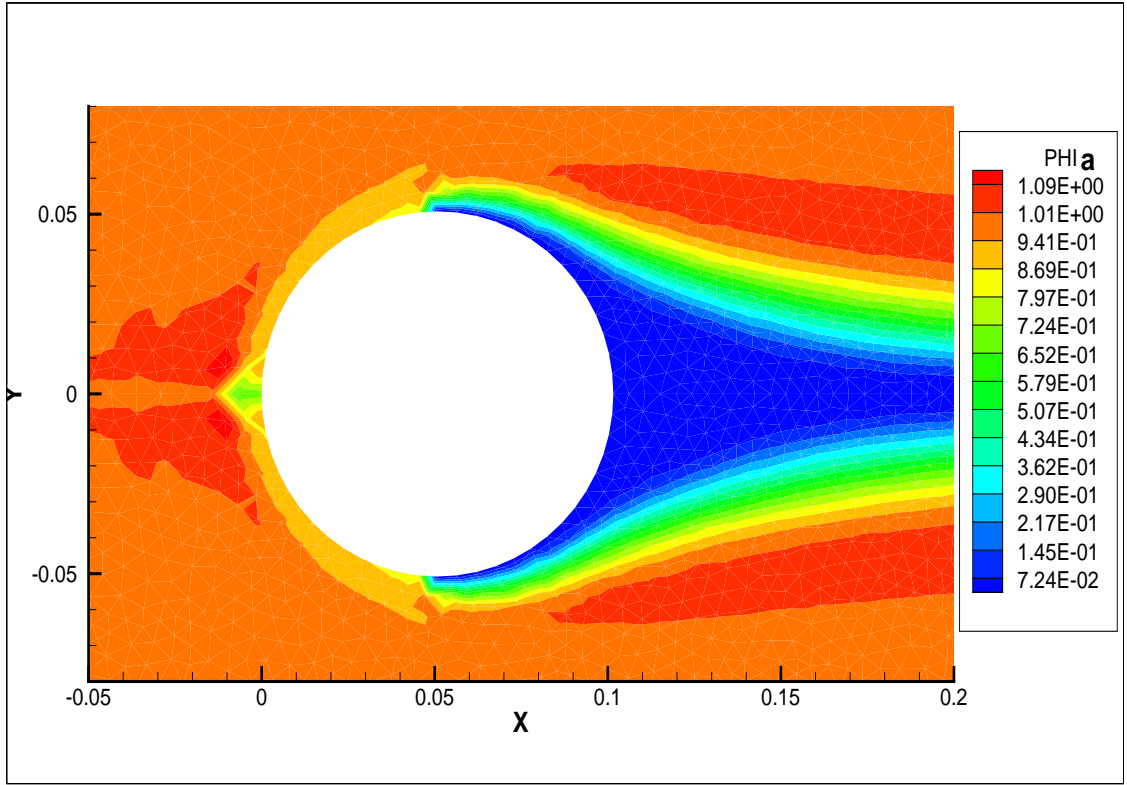

Figura 6.17: Transporte do escalar $\phi$ : malha $m_{4}$ - 6724 vértices, reconstrução linear

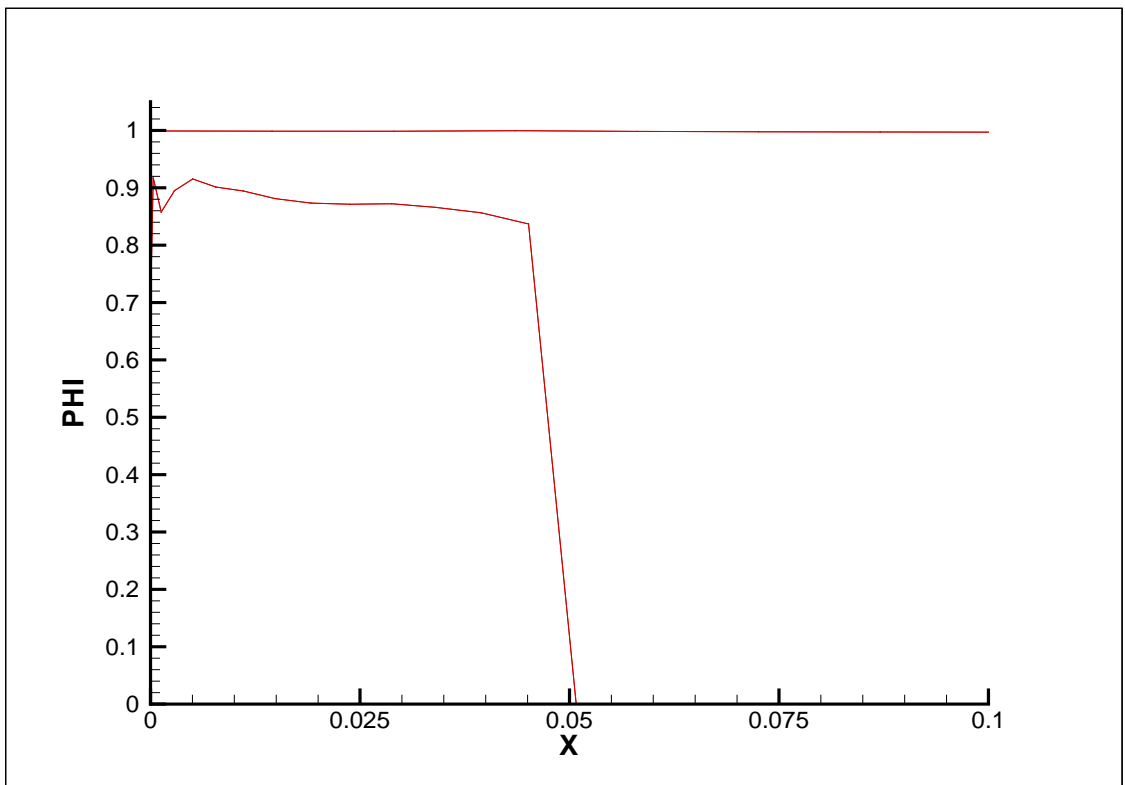

Figura 6.18: Valores da propriedade $\phi$ no cilindro: malha $m 4$ - 6724 vértices, reconstrução linear. 


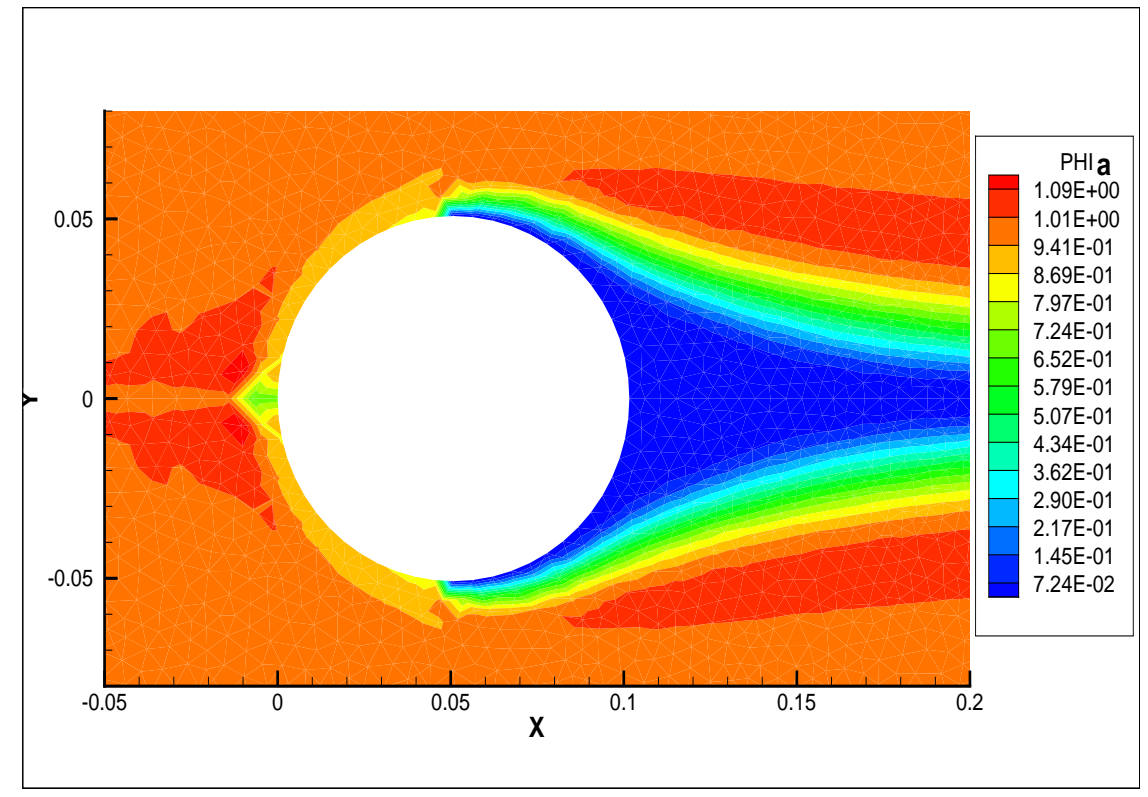

Figura 6.19: Transporte da propriedade $\phi$ : malha $m_{4}-6724$ vértices, reconstrução quadrática.

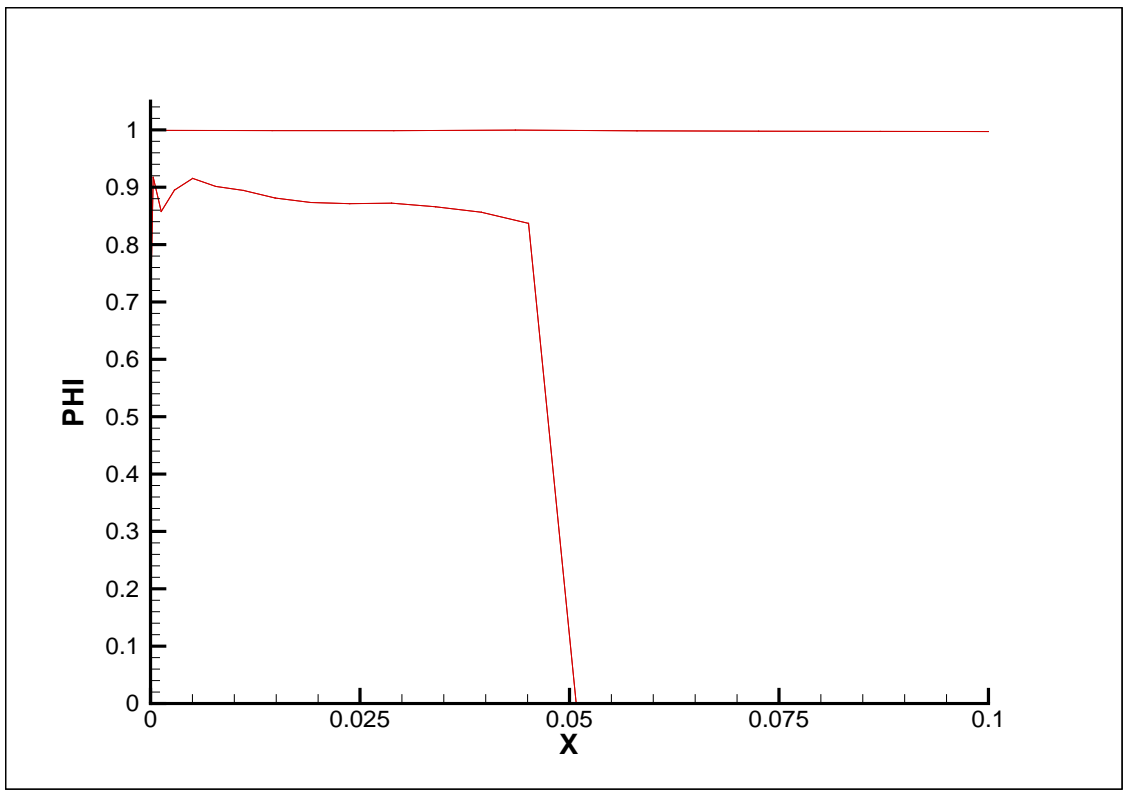

Figura 6.20: Valores da propriedade $\phi$ no cilindro: malha $m 4$ - 6724 vértices, reconstrução quadrática. 


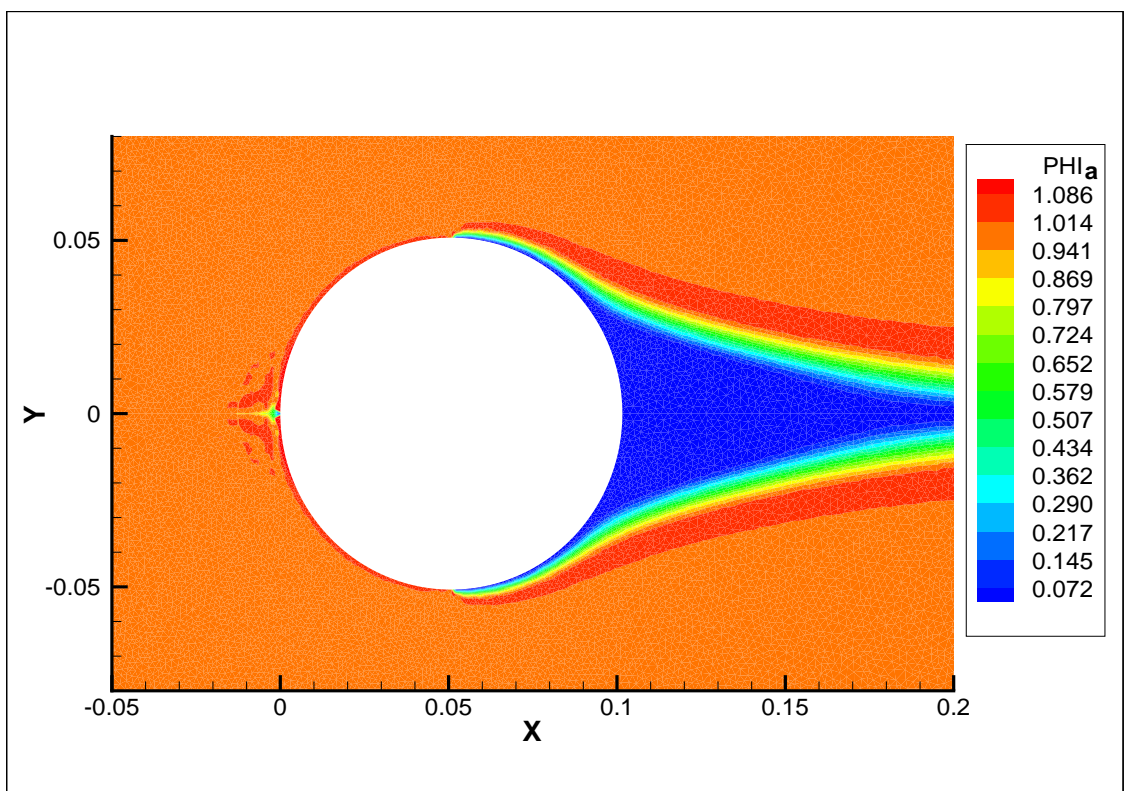

(a)

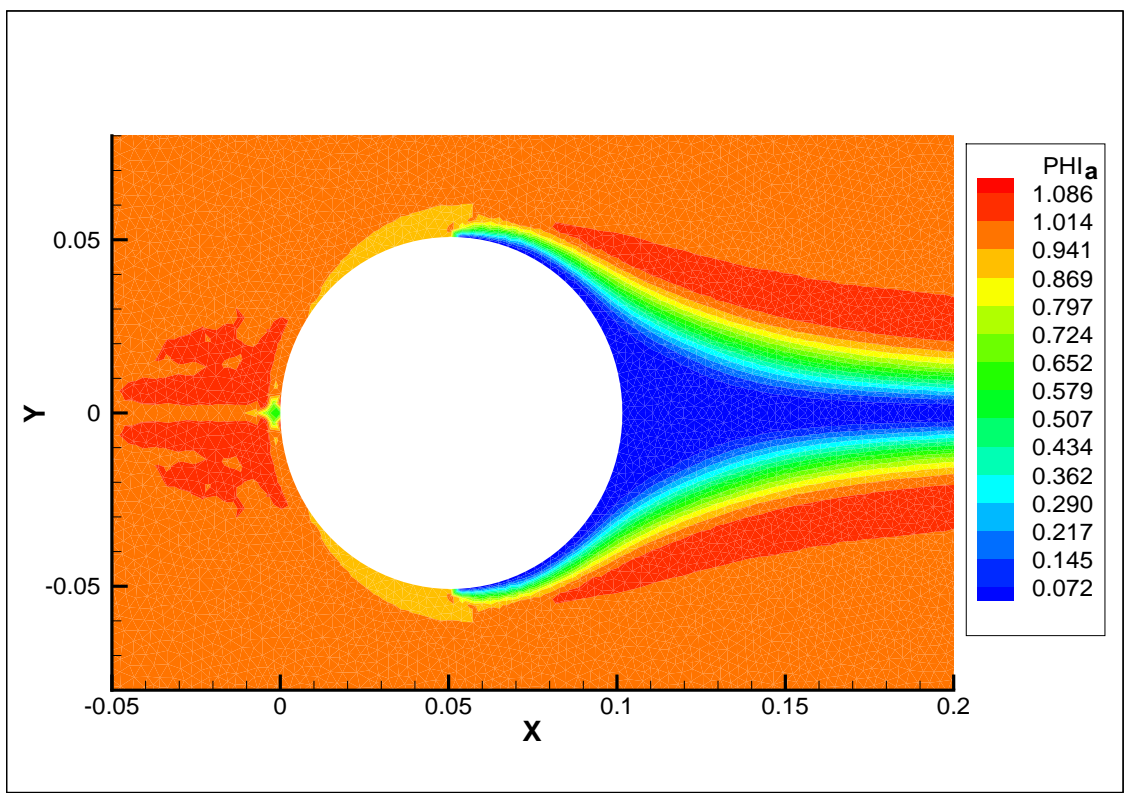

(b)

Figura 6.21: Transporte do escalar $\phi$ : (a) malha m2 - 13664 vértices, reconstrução linear; (b) malha $m 5$ - 26866 vértices, reconstrução linear. 


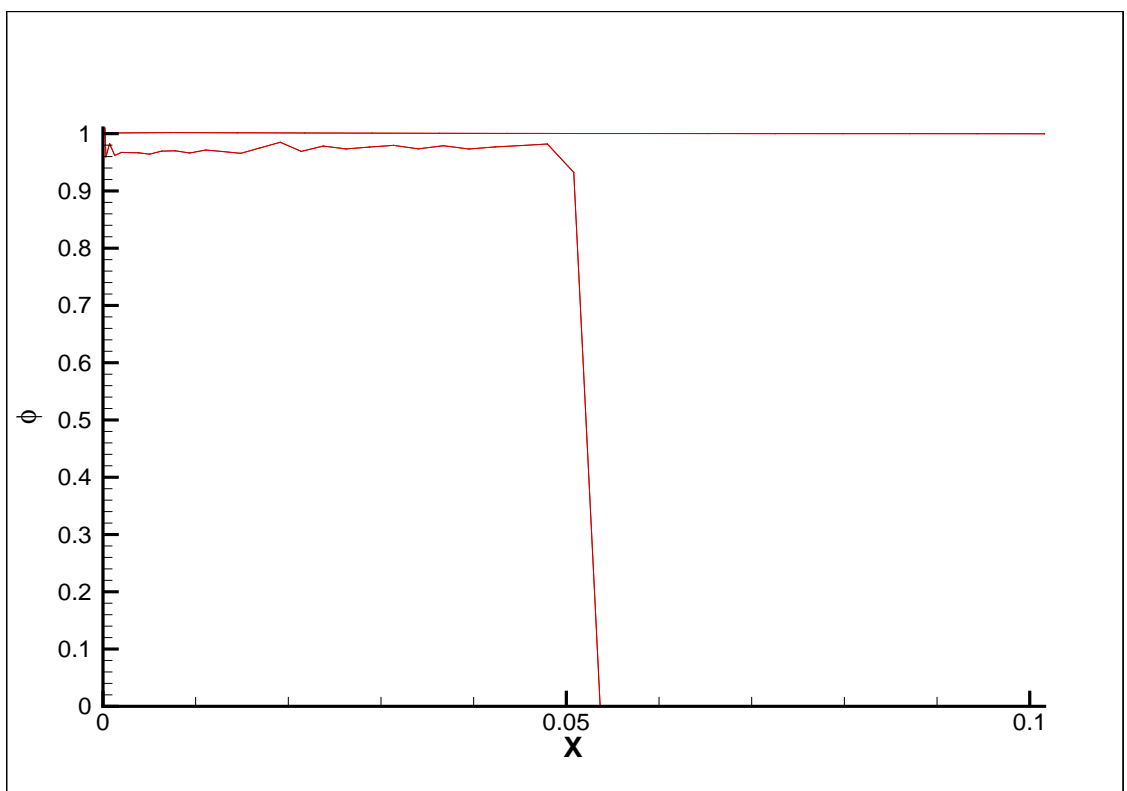

(a)

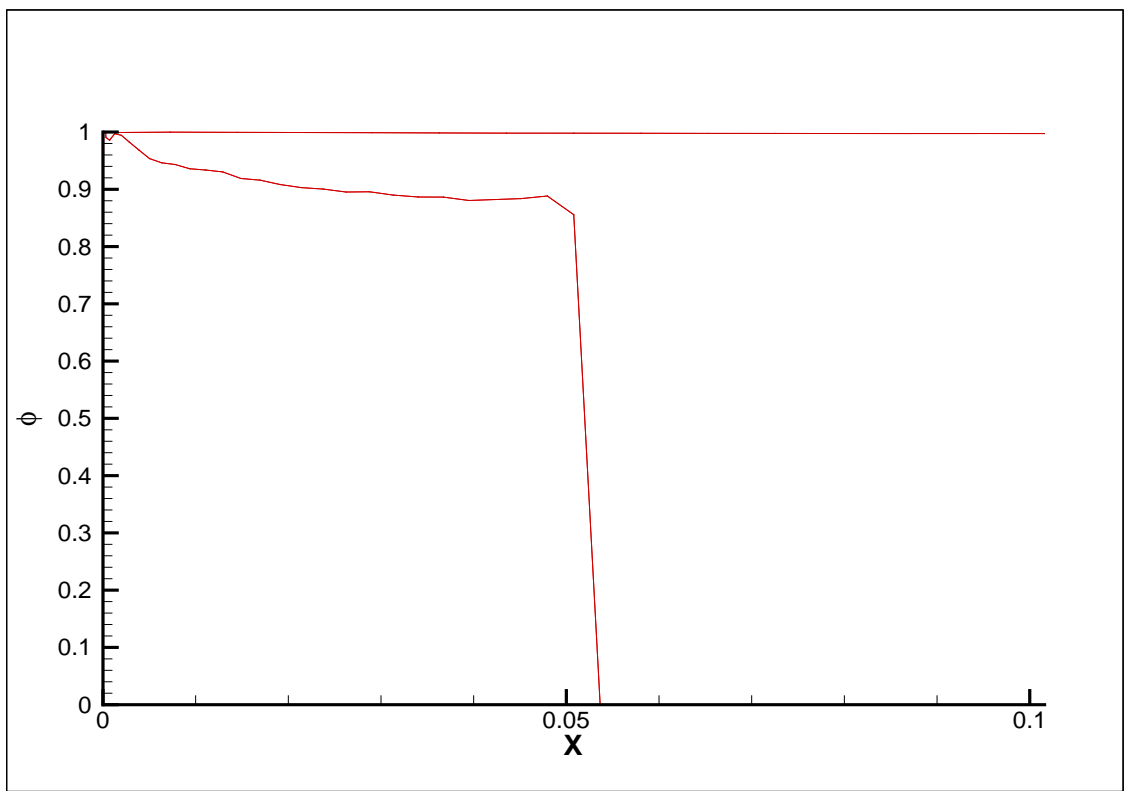

(b)

Figura 6.22: Valores da propriedade $\phi$ no cilindro: (a) malha m2 - 13664 vértices, reconstrução linear; (b) malha m5 - 26866 vértices, reconstrução linear. 


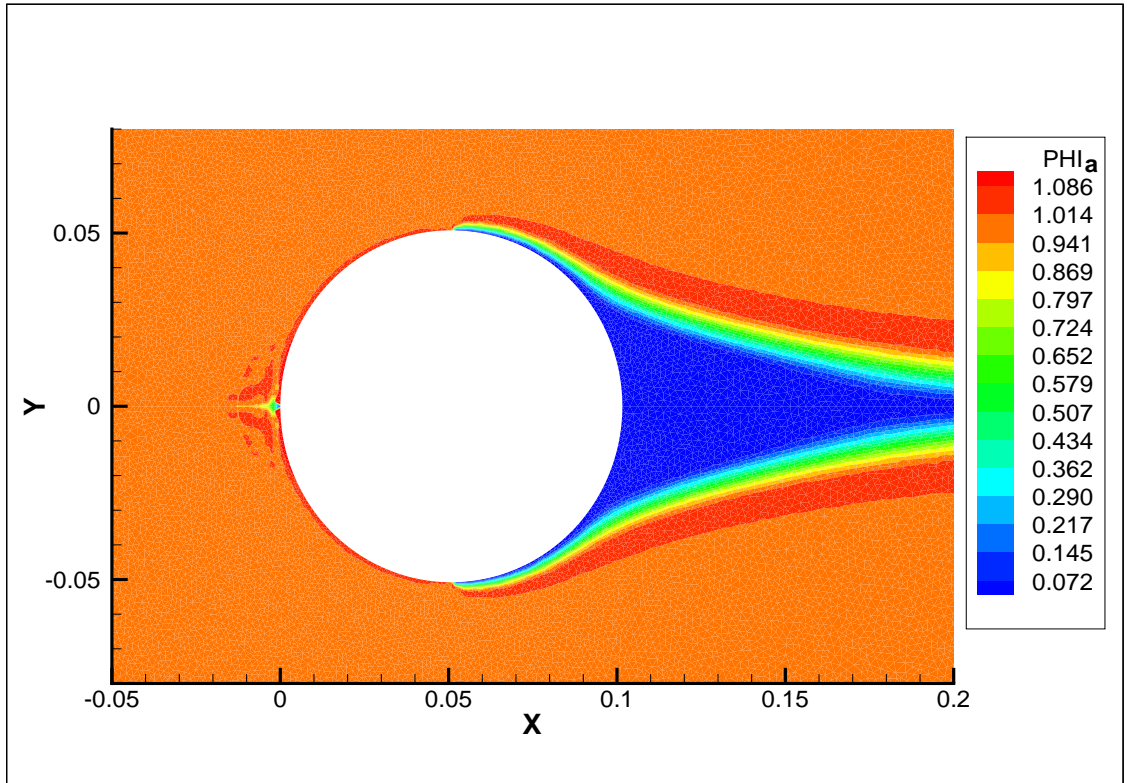

Figura 6.23: Transporte do escalar $\phi$ : malha m3 - 55239 vértices, reconstrução linear.

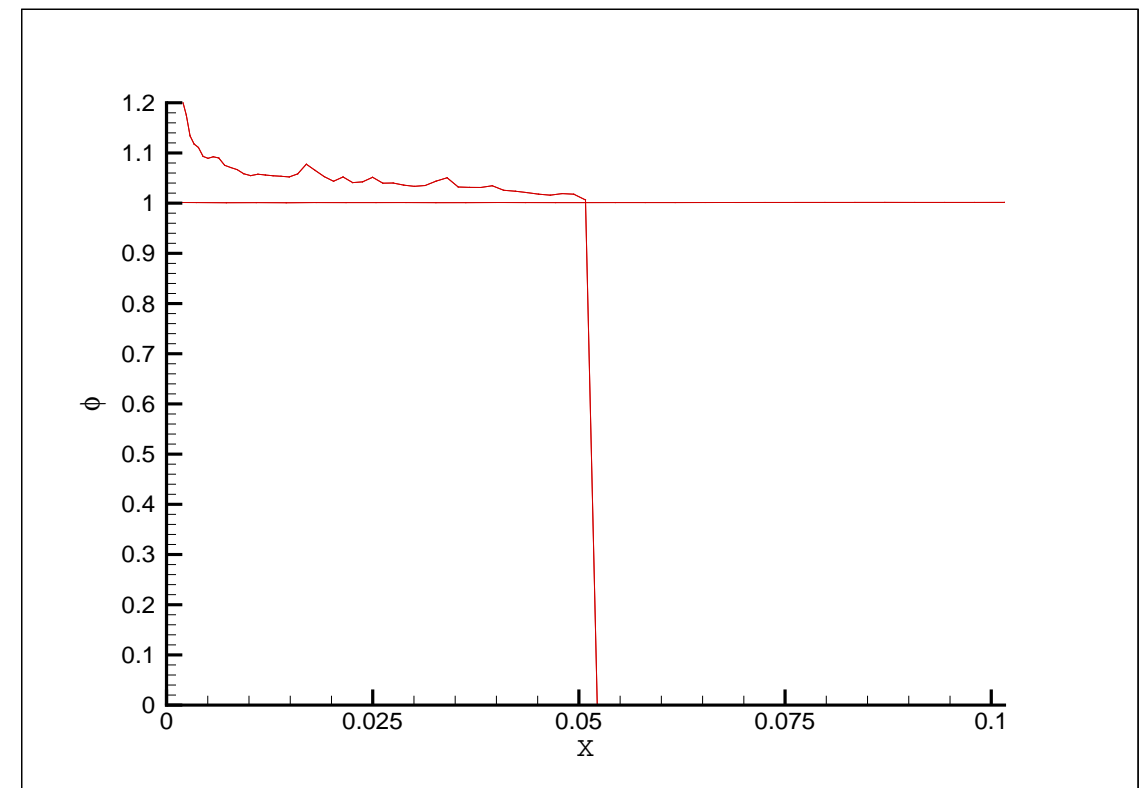

Figura 6.24: Valores da propriedade $\phi$ no cilindro: malha m3 - 55239 vértices, reconstrução linear. 
De um modo geral, refinando-se a malha ou aumentando a ordem do polinômio de reconstrução vê-se o detalhe da influência do campo de velocidade na frente do cilindro e a presença de overshoot na descontinuidade e o comportamento da difusão numérica na região atrás do cilindro.

Para uma análise da difusão numérica, com condição de contorno definida pela componente da normal $n_{x}$, traçou-se os perfis $p_{1}, p_{2}$ e $p_{3}$ apresentados na Figura 6.25, cujas abscissas são, respectivamente, $x=0.0, x=0.12$ e $x=0.16$.

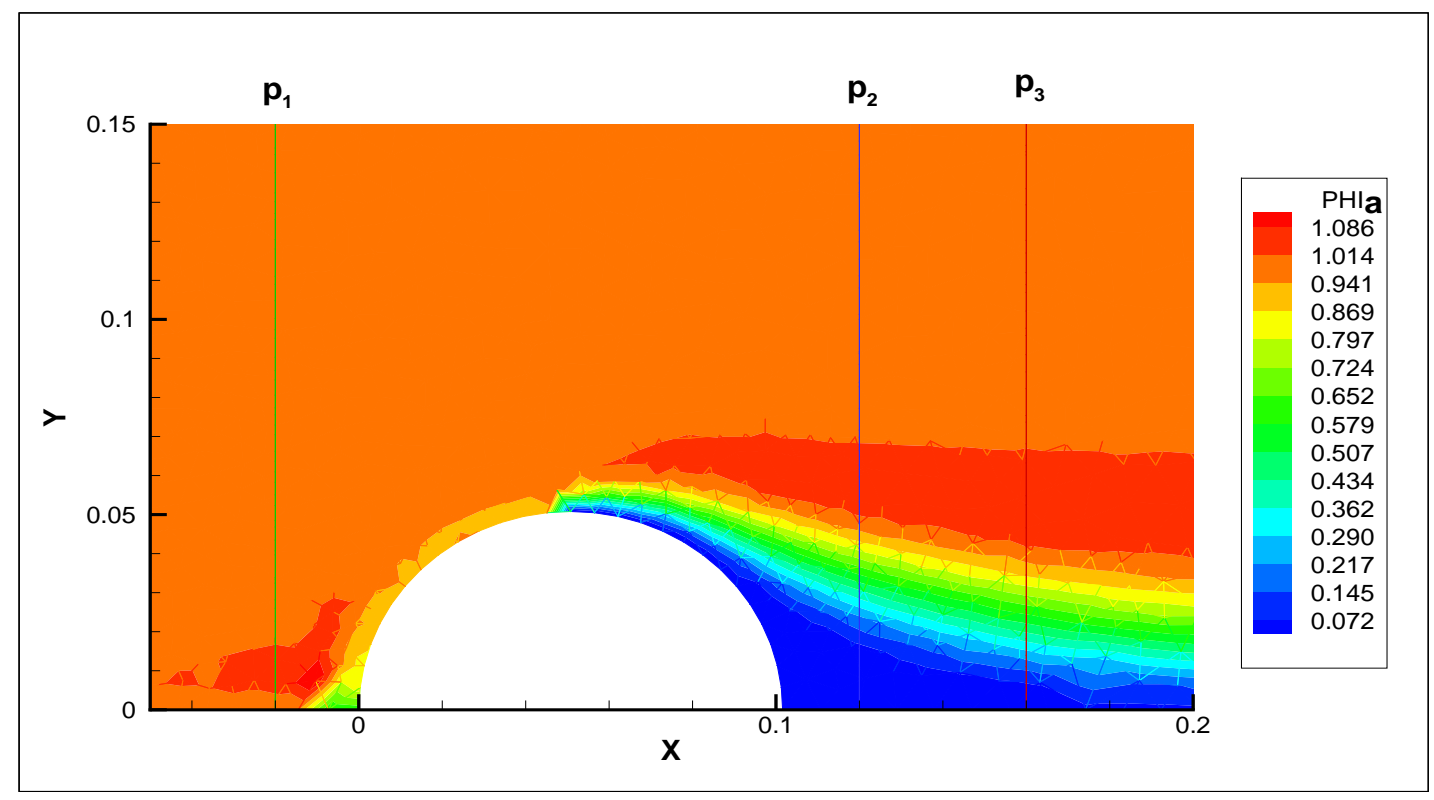

Figura 6.25: Perfis $p_{1}, p_{2}$ e $p_{3}$ no transporte da propriedade $\phi$, com condição de contorno definido pela componente da normal $n_{x}$. 


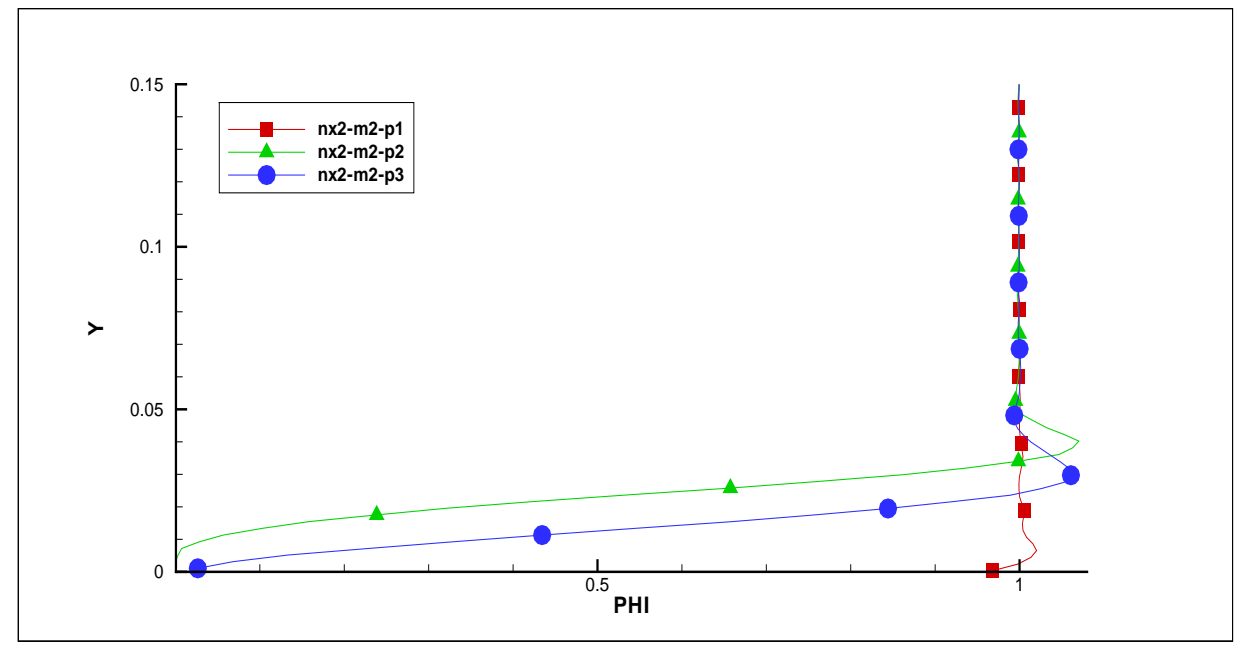

(a)

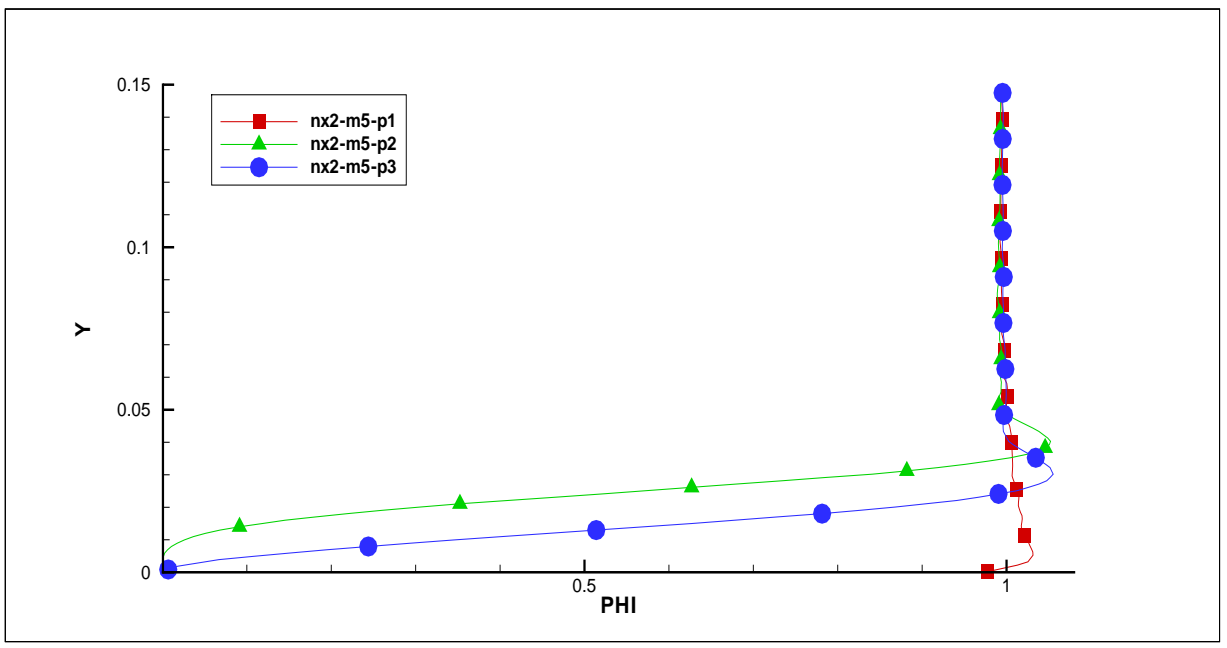

(b)

Figura 6.26: Perfis $p_{1}, p_{2}$ e $p_{3}$ no transporte da propriedade $\phi$ - campo de velocidade do NSC2KE: (a) malha m2 (13664 vértices), reconstrução linear; (b) malha m5 (26866 vértices), reconstrução quadrática. 


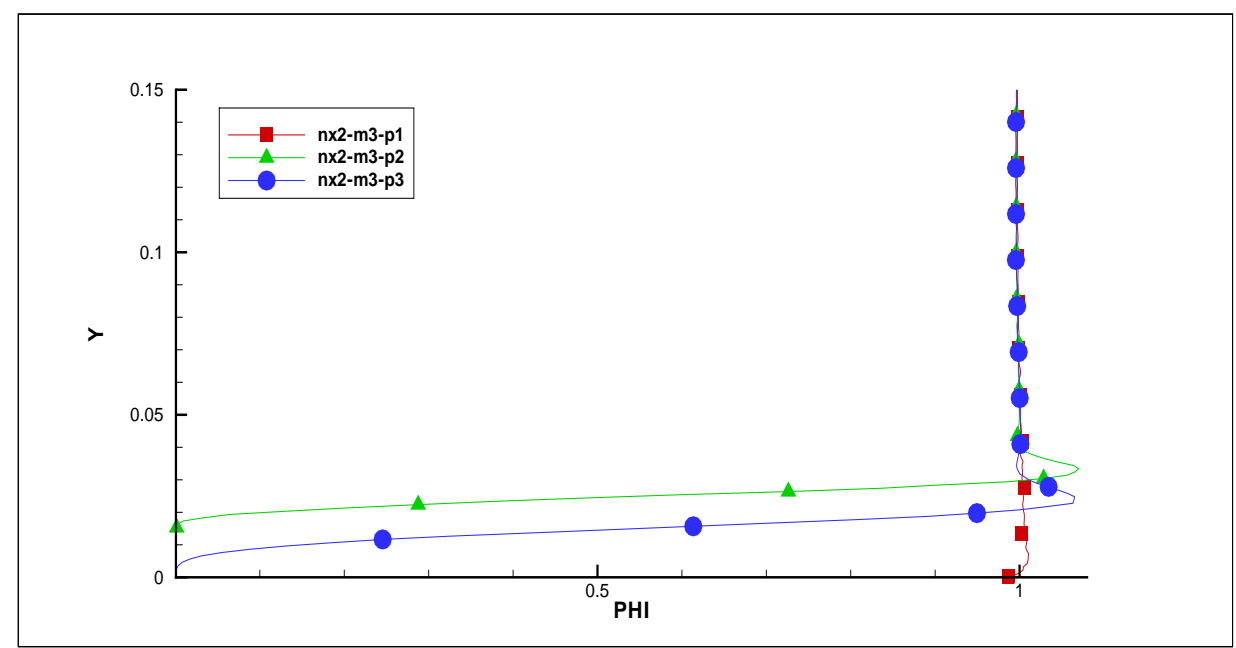

Figura 6.27: Perfis $p_{1}, p_{2}$ e $p_{3}$ no transporte da propriedade $\phi$ - campo de velocidade do NSC2KE: malha m3 (55239 vértices), reconstrução linear.

Analisando os gráficos das Figuras 6.26-6.27 com a Tabela 6.2, percebe-se um ganho de tempo, reduzindo a difusão numérica ao refinar a região atrás do cilindro, Figura 6.28. Observa-se a redução da difusão numérica e ganho no tempo, na malha m2 gastou-se 821s enquanto que em malha $m_{4}$ gastou-se apenas 321s.

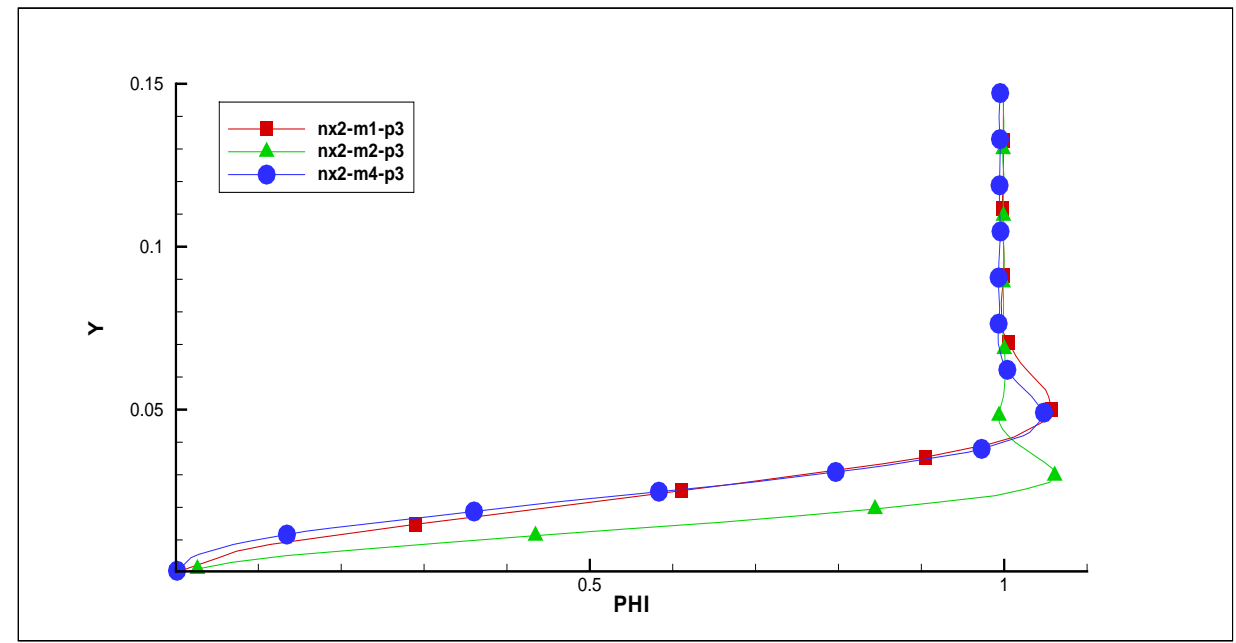

Figura 6.28: Perfil $p_{3}$ no transporte da propriedade $\phi$ nas malhas $m 1, m 2$ e $m 3$, reconstrução quadrática: influência da malha na difusão numérica. 


\subsection{Transporte da propriedade $\phi$ - velocidade cons- tante $V=(1,0)$}

Neste teste com o cilindro circular na região retangular, usou-se as componentes da velocidade constantes em todos os nós da malha, componente $\mathrm{x}$ da velocidade igual a $1 \mathrm{e}$ componente y da velocidade igual a 0 , buscando analisar o efeito da difusão numérica na região de sombra.

A condição para determinar a região de sombra é feita com base na componente normal $n_{x}$.

Nas Tabelas 6.3 - 6.8, são apresentados os tempos de execução dos testes para as malhas $m 1$ - $m 6$ e o número de iterações no transporte da propriedade $\phi$ que simula o transporte das gotículas.

\begin{tabular}{|c|c|c|}
\hline Ordem de Reconstrução & tempo (s) & no. de iterações \\
\hline 2 & 117 & 742 \\
\hline 3 & 289 & 947 \\
\hline 4 & 1493 & 710 \\
\hline
\end{tabular}

Tabela 6.3: Transporte da propriedade $\phi$ na malha $m 1,3559$ vértices: tempo de execução e número de iterações.

\begin{tabular}{|c|c|c|}
\hline Ordem de Reconstrução & tempo (s) & no. de iterações \\
\hline 2 & 835 & 1339 \\
\hline 3 & 1559 & 1348 \\
\hline 4 & 11488 & 1355 \\
\hline
\end{tabular}

Tabela 6.4: Transporte da propriedade $\phi$ na malha m2, 13664 vértices: tempo de execução e número de iterações. 


\begin{tabular}{|c|c|c|}
\hline Ordem de Reconstrução & tempo (s) & no. de iterações \\
\hline 2 & 12004 & 2597 \\
\hline 3 & 16443 & 2593 \\
\hline 4 & 93556 & 2660 \\
\hline
\end{tabular}

Tabela 6.5: Transporte da propriedade $\phi$ na malha m3, 55239 vértices: tempo de execução e número de iterações.

\begin{tabular}{|c|c|c|}
\hline Ordem de Reconstrução & tempo (s) & no. de iterações \\
\hline 2 & 272 & 944 \\
\hline 3 & 623 & 952 \\
\hline 4 & 3883 & 954 \\
\hline
\end{tabular}

Tabela 6.6: Transporte da propriedade $\phi$ na malha $m 4,6724$ vértices: tempo de execução e número de iterações.

\begin{tabular}{|c|c|c|}
\hline Ordem de Reconstrução & tempo (s) & no. de iterações \\
\hline 2 & 2432 & 1803 \\
\hline 3 & 5135 & 1805 \\
\hline 4 & 31273 & 1860 \\
\hline
\end{tabular}

Tabela 6.7: Transporte da propriedade $\phi$ na malha $m 5,26866$ vértices: tempo de execução e número de iterações.

\begin{tabular}{|c|c|c|}
\hline Ordem de Reconstrução & tempo (s) & no. de iterações \\
\hline 2 & 11321 & 2557 \\
\hline 3 & 16533 & 2602 \\
\hline 4 & 85098 & 2622 \\
\hline
\end{tabular}

Tabela 6.8: Transporte da propriedade $\phi$ na malha $m 6,76013$ vértices: tempo de execução e número de iterações. 
As Figuras 6.29 - 6.46 mostram o transporte da propriedade $\phi$ para cada ordem de reconstrução para a análise do transporte da propriedade $\phi$ e o comportamento da difusão numérica.

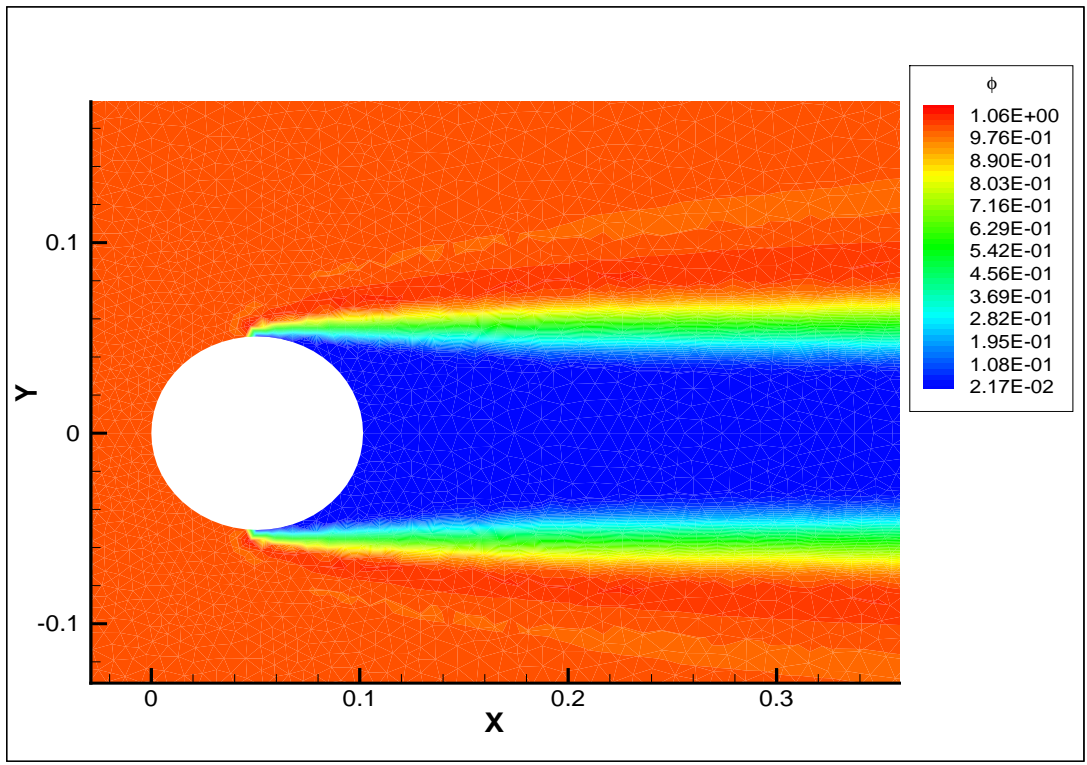

Figura 6.29: Transporte da propriedade $\phi$ na malha $m 1,3559$ vértices: reconstrução linear.

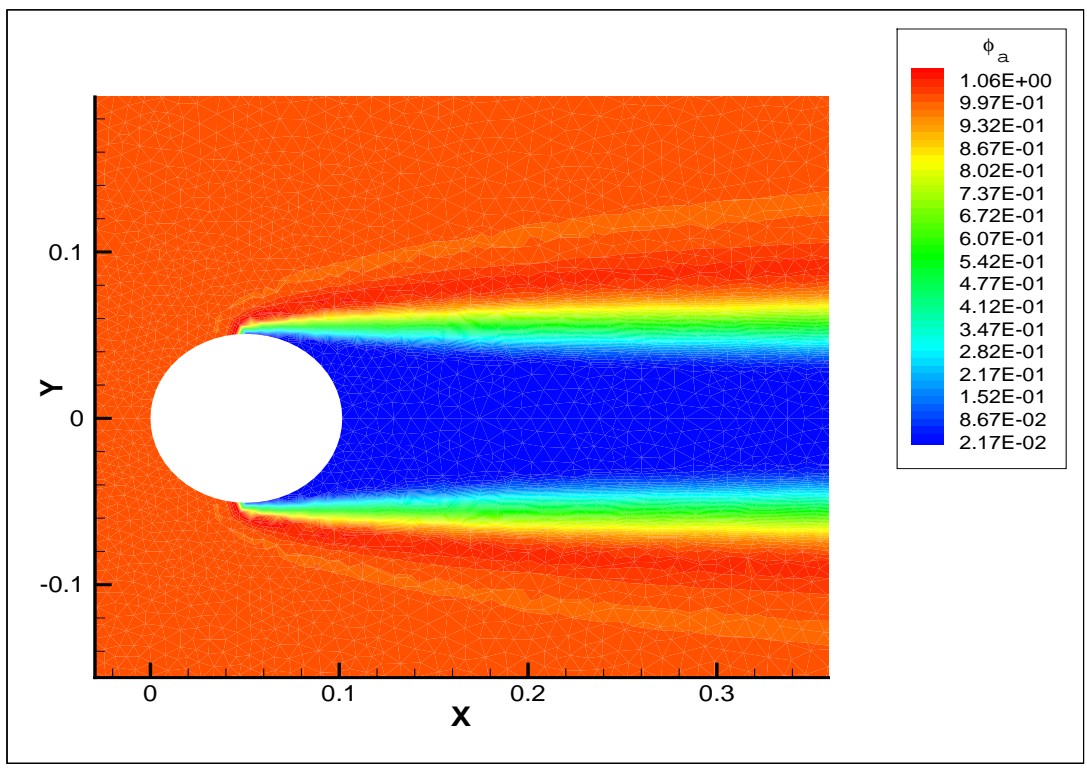

Figura 6.30: Transporte da propriedade $\phi$ na malha $m 1,3559$ vértices: reconstrução quadrática. 


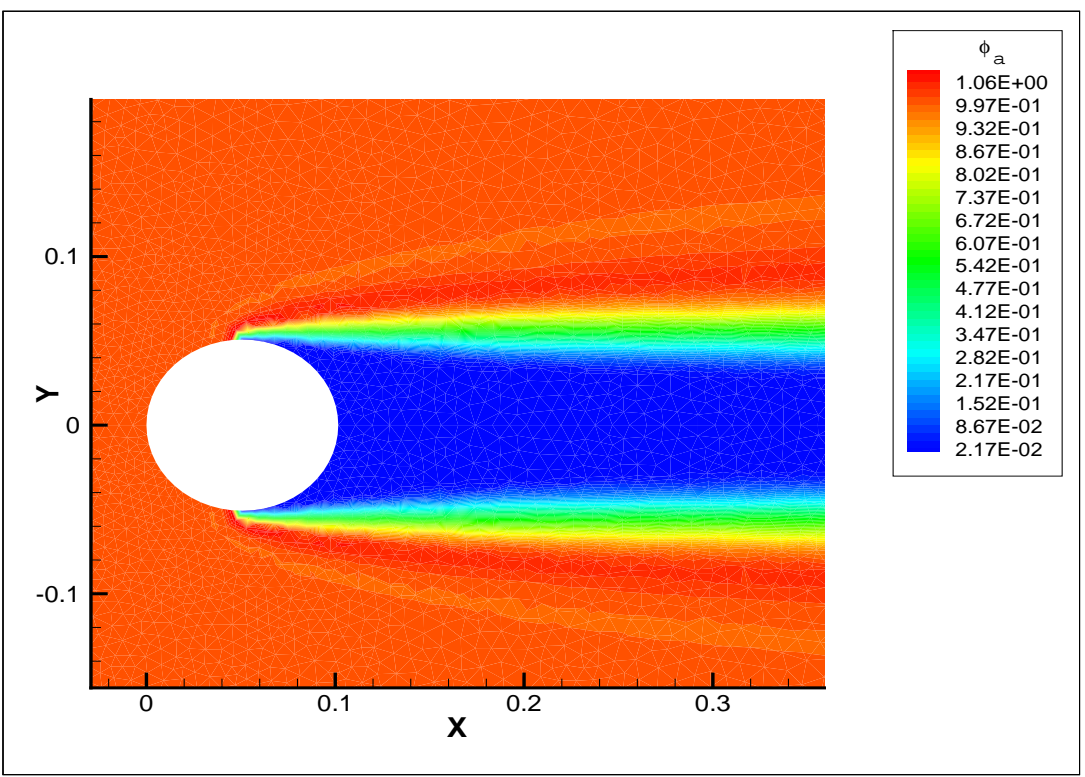

Figura 6.31: Transporte da propriedade $\phi$ na malha $m 1,3559$ vértices: reconstrução cúbica.

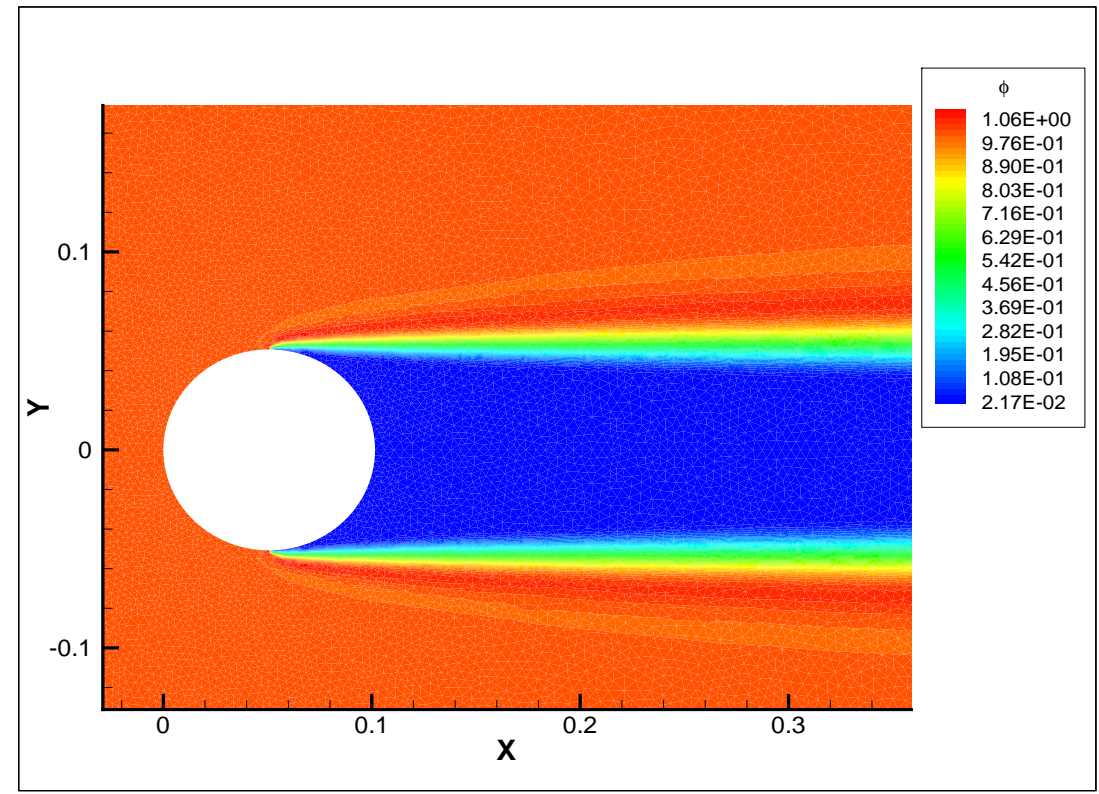

Figura 6.32: Transporte da propriedade $\phi$ na malha m2, 13664 vértices: reconstrução linear. 


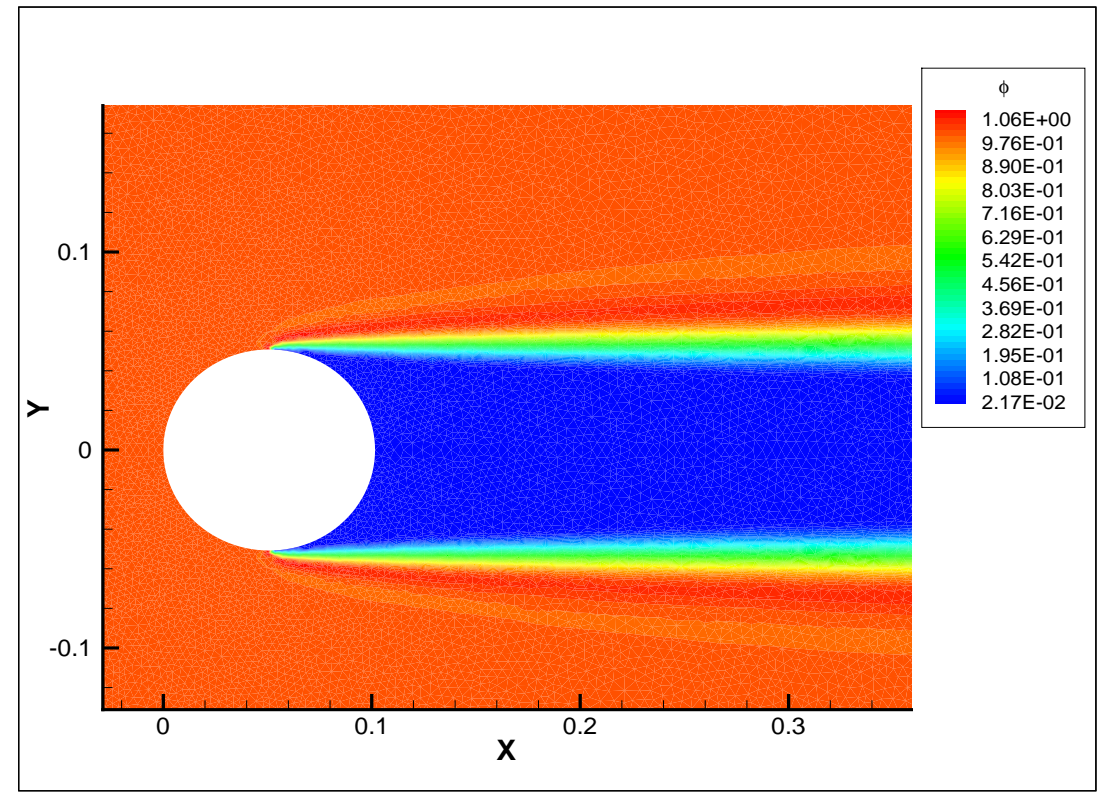

Figura 6.33: Transporte da propriedade $\phi$ na malha m2, 13664 vértices: reconstrução quadrática.

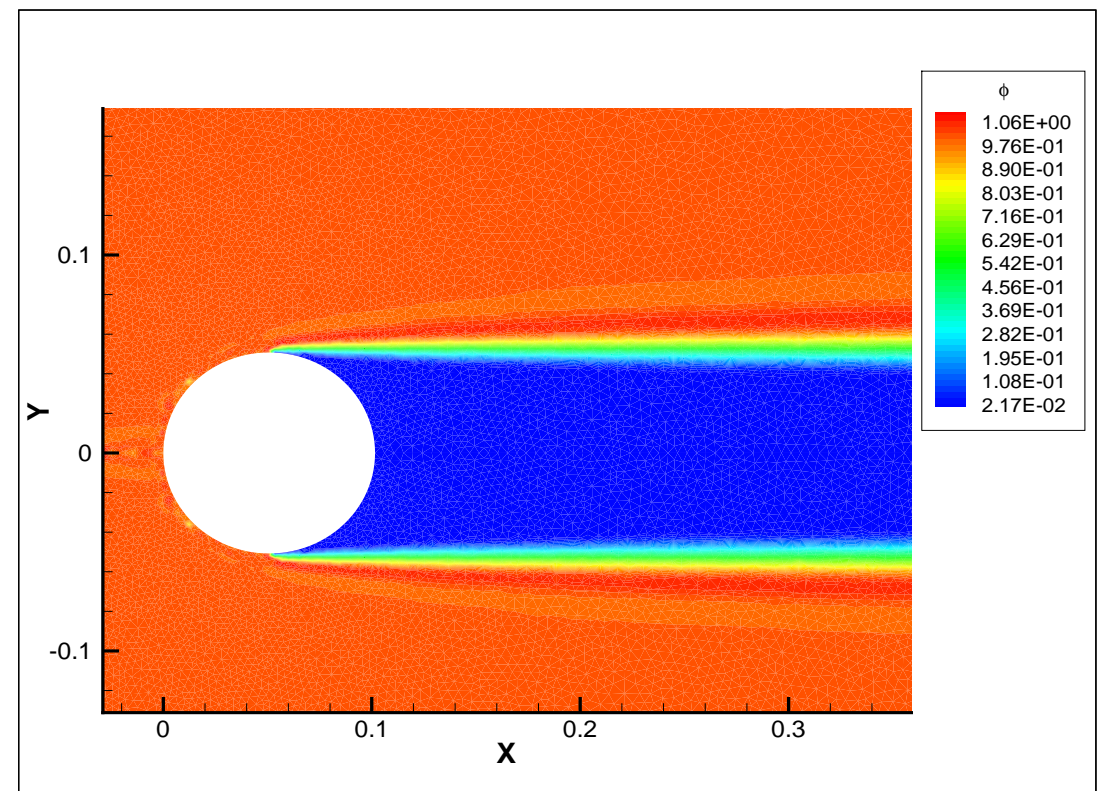

Figura 6.34: Transporte da propriedade $\phi$ na malha m2, 13664 vértices: reconstrução cúbica. 


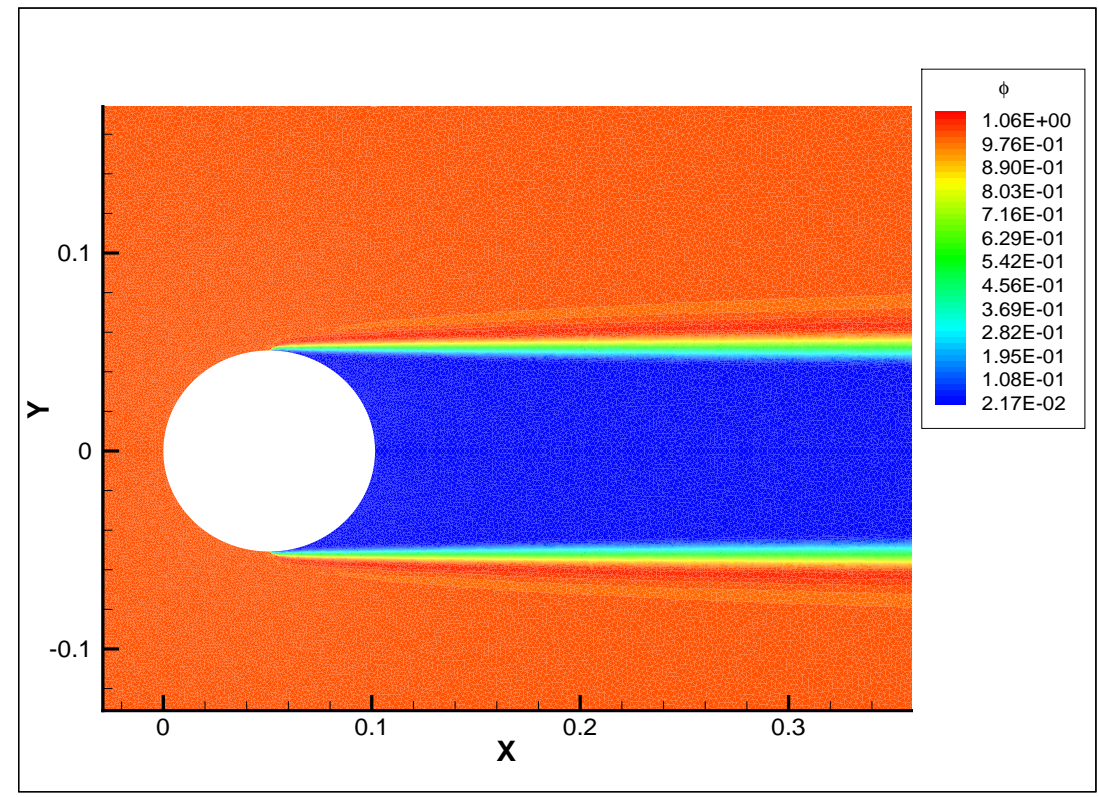

Figura 6.35: Transporte da propriedade $\phi$ na malha m3, 55239 vértices: reconstrução linear.

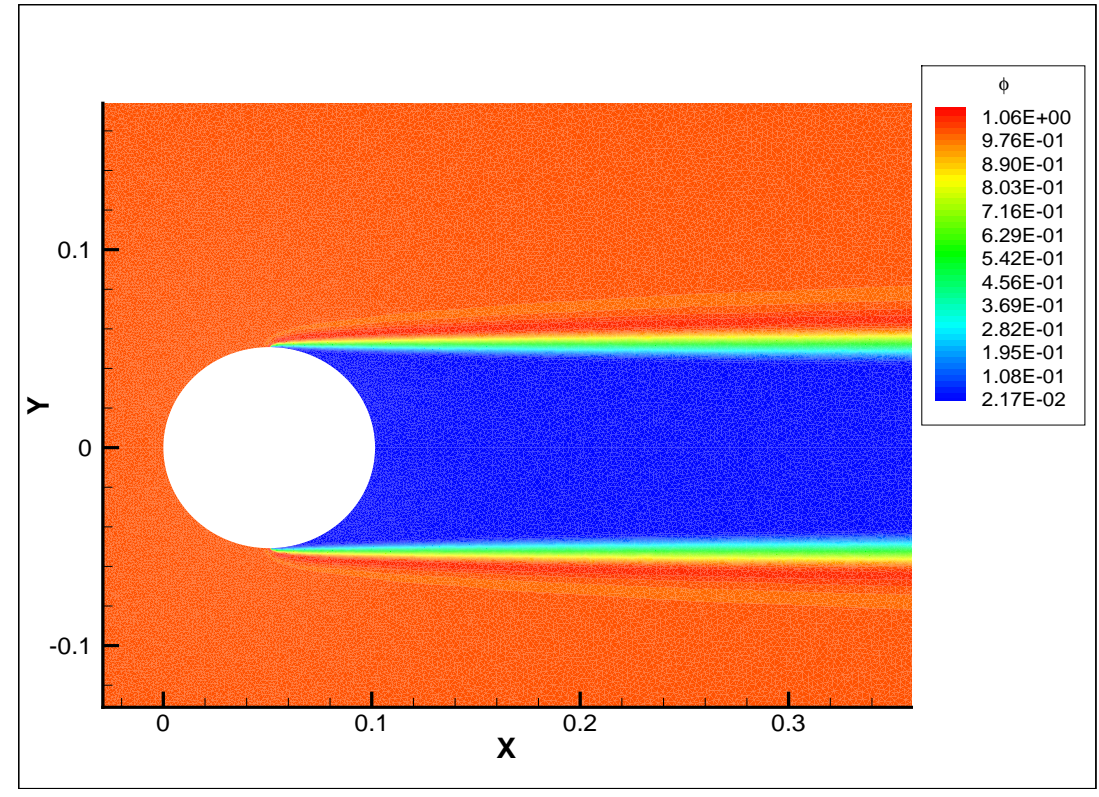

Figura 6.36: Transporte da propriedade $\phi$ na malha m3, 55239 vértices: reconstrução quadrática. 


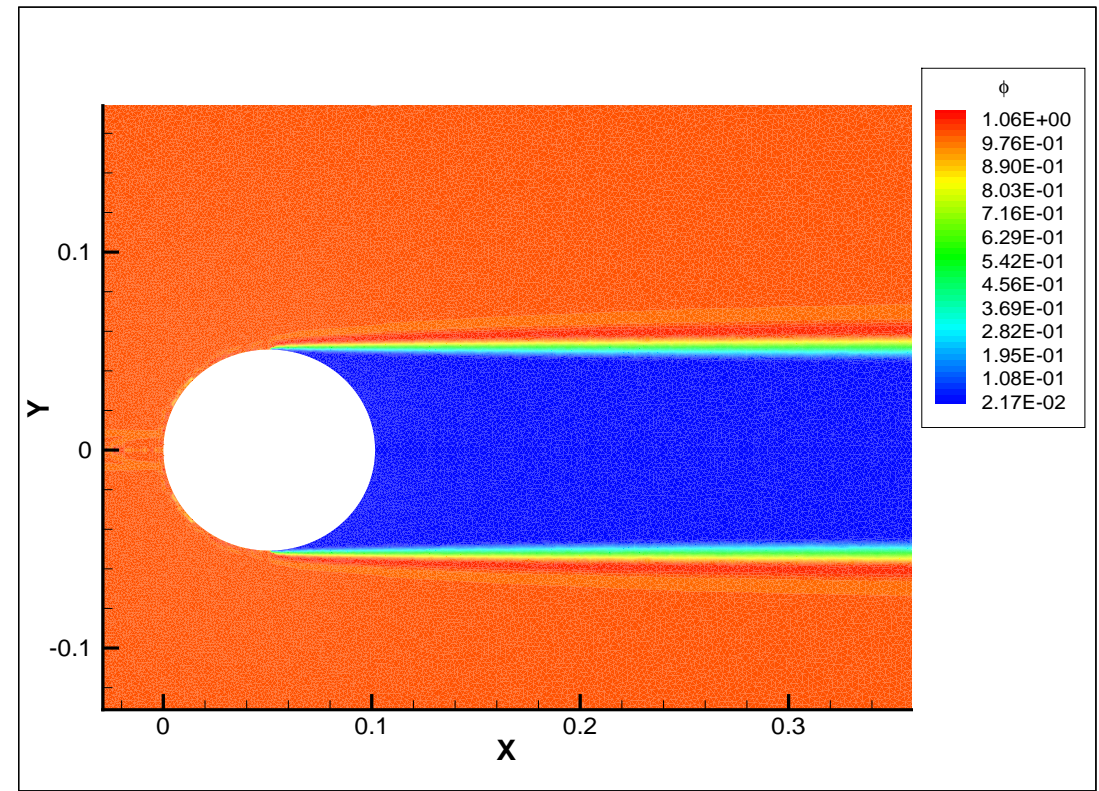

Figura 6.37: Transporte da propriedade $\phi$ na malha m3, 55239 vértices: reconstrução cúbica.

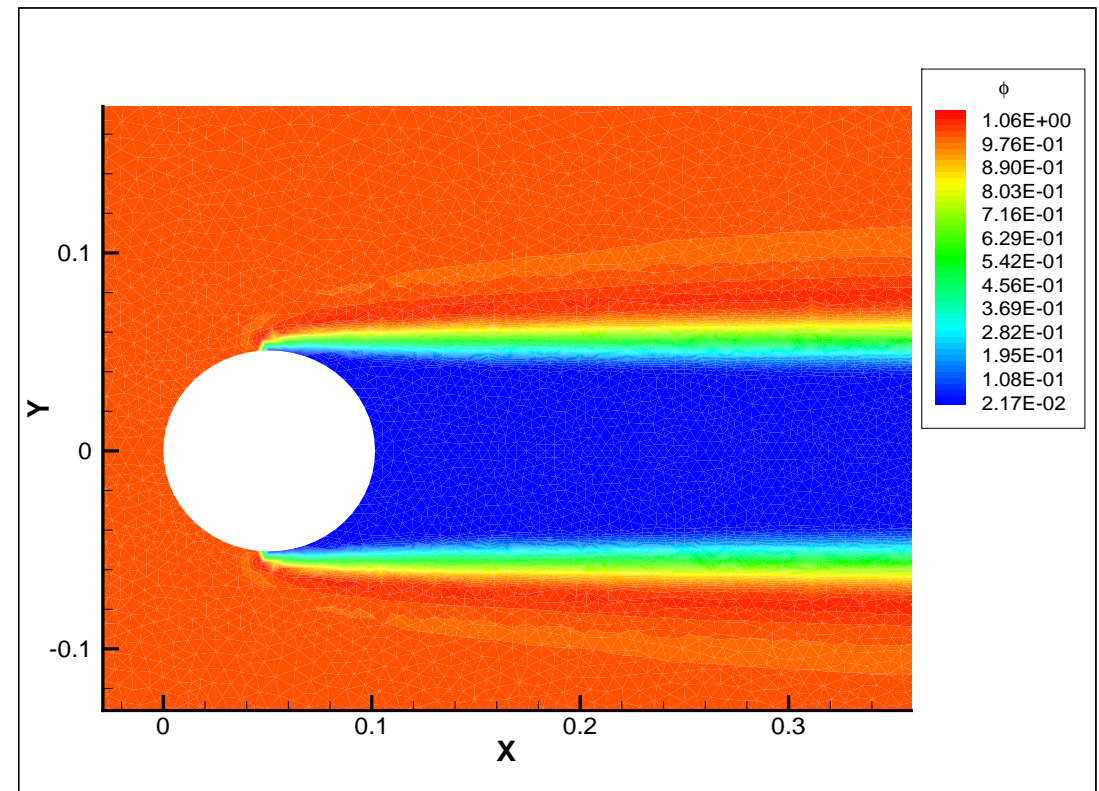

Figura 6.38: Transporte da propriedade $\phi$ na malha $m 4$, 6724 vértices: reconstrução linear. 


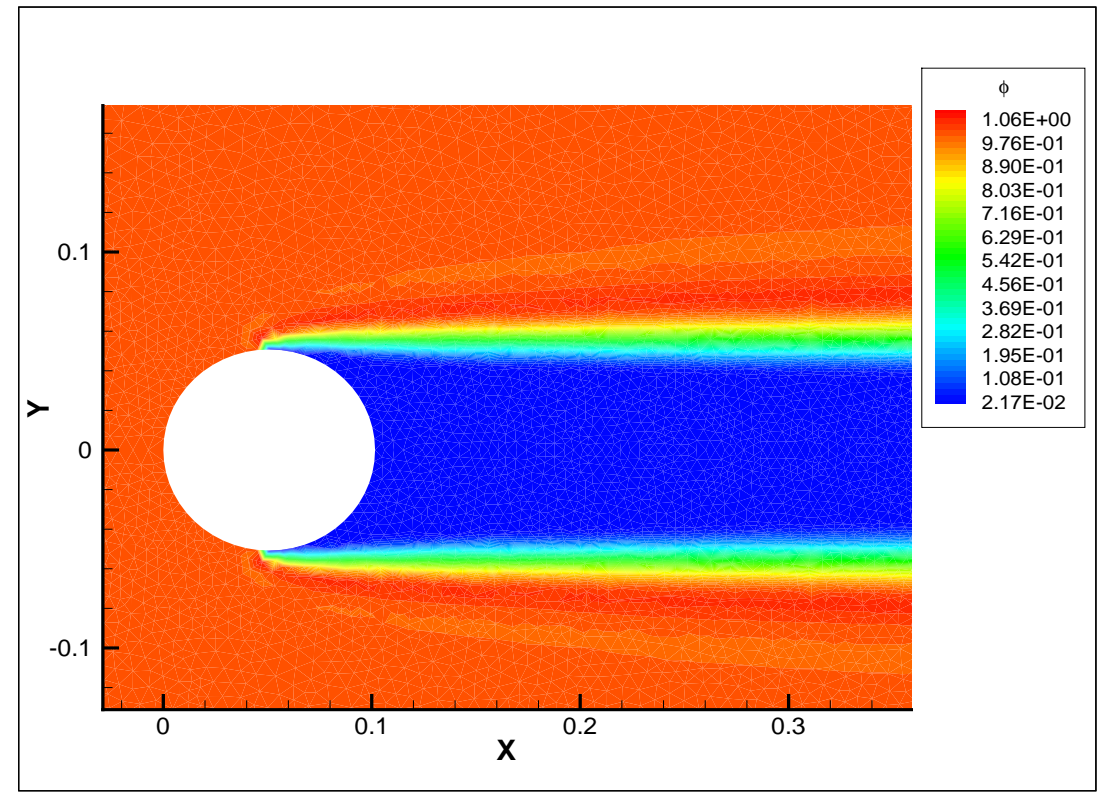

Figura 6.39: Transporte da propriedade $\phi$ na malha $m_{4}$, 6724 vértices: reconstrução quadrática.

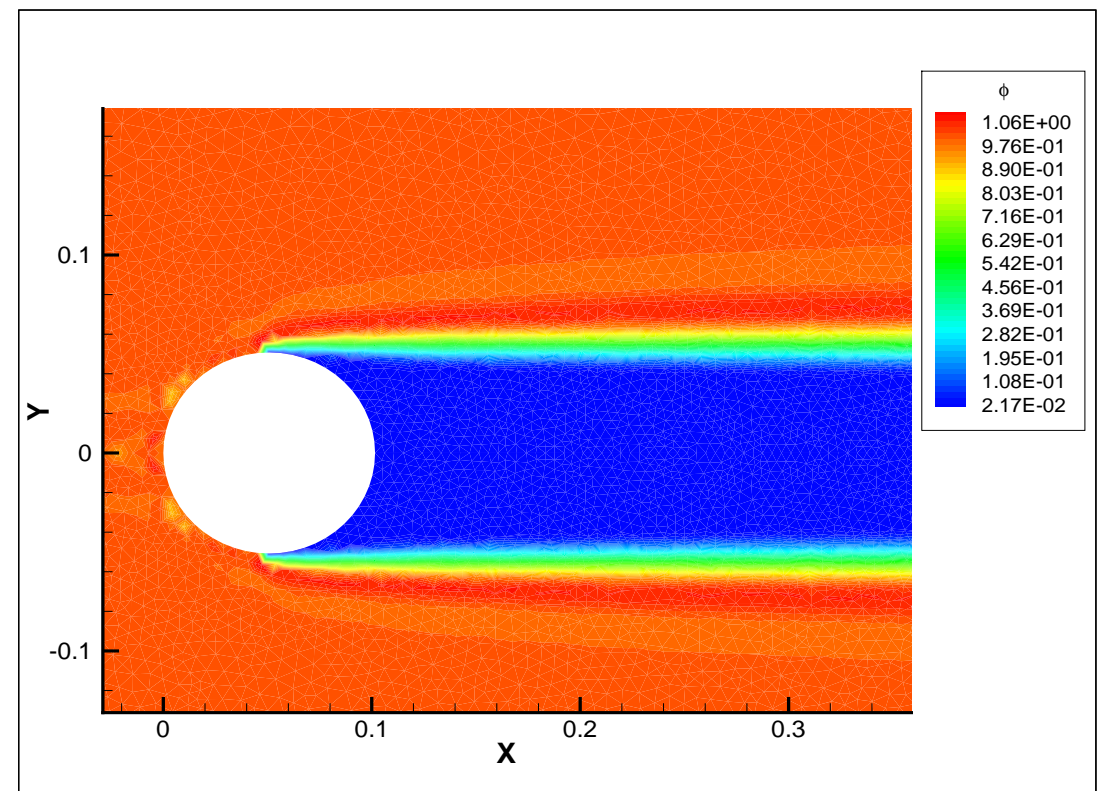

Figura 6.40: Transporte da propriedade $\phi$ na malha $m 4$, 6724 vértices: reconstrução cúbica. 


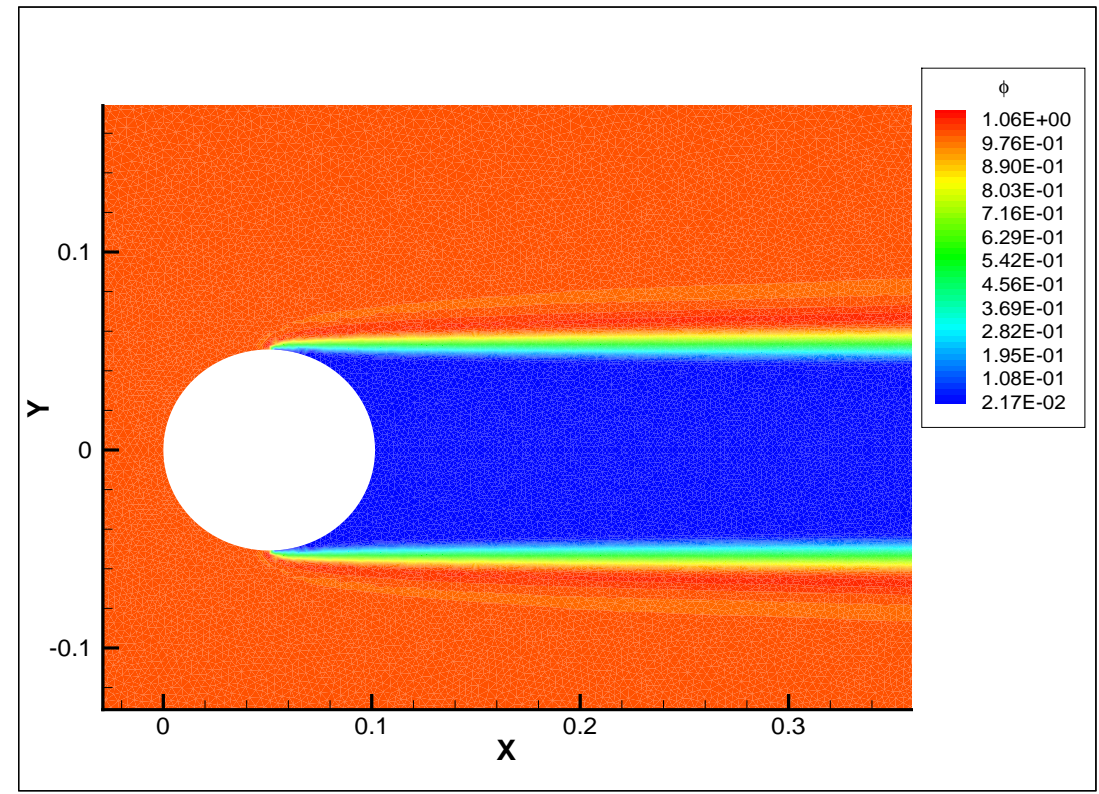

Figura 6.41: Transporte da propriedade $\phi$ na malha $m 5,26866$ vértices: reconstrução linear.

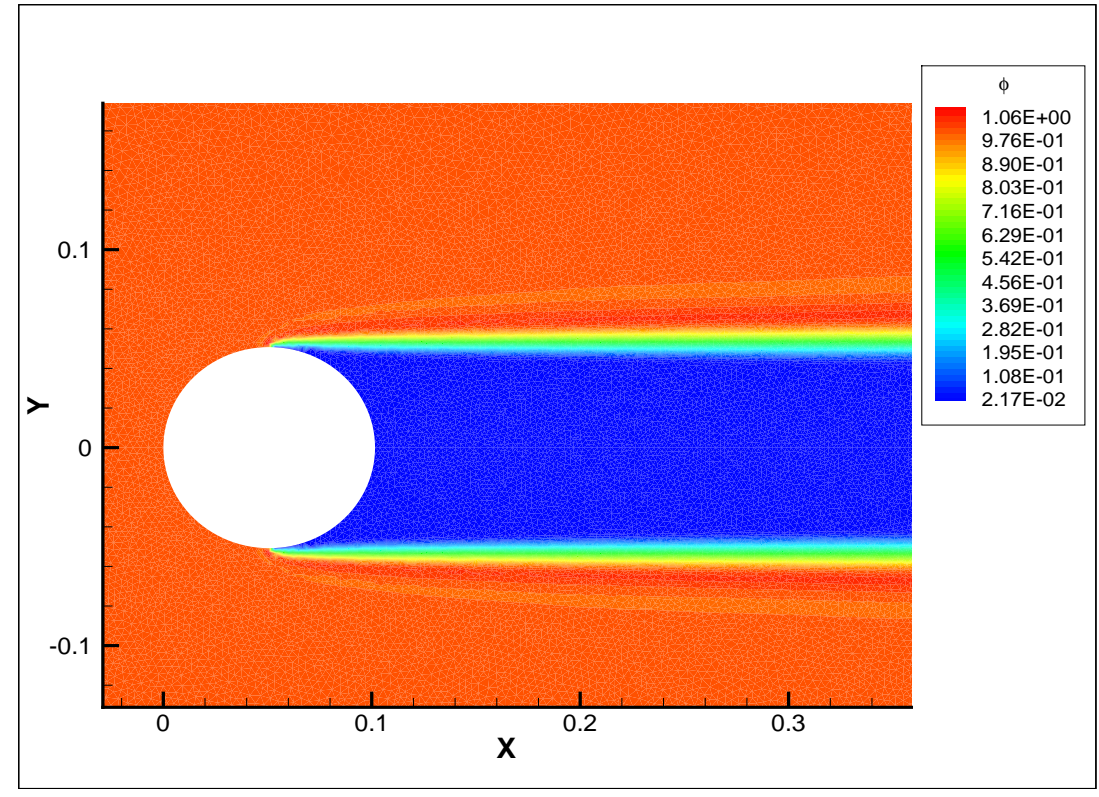

Figura 6.42: Transporte da propriedade $\phi$ na malha $m 5,26866$ vértices: reconstrução quadrática. 


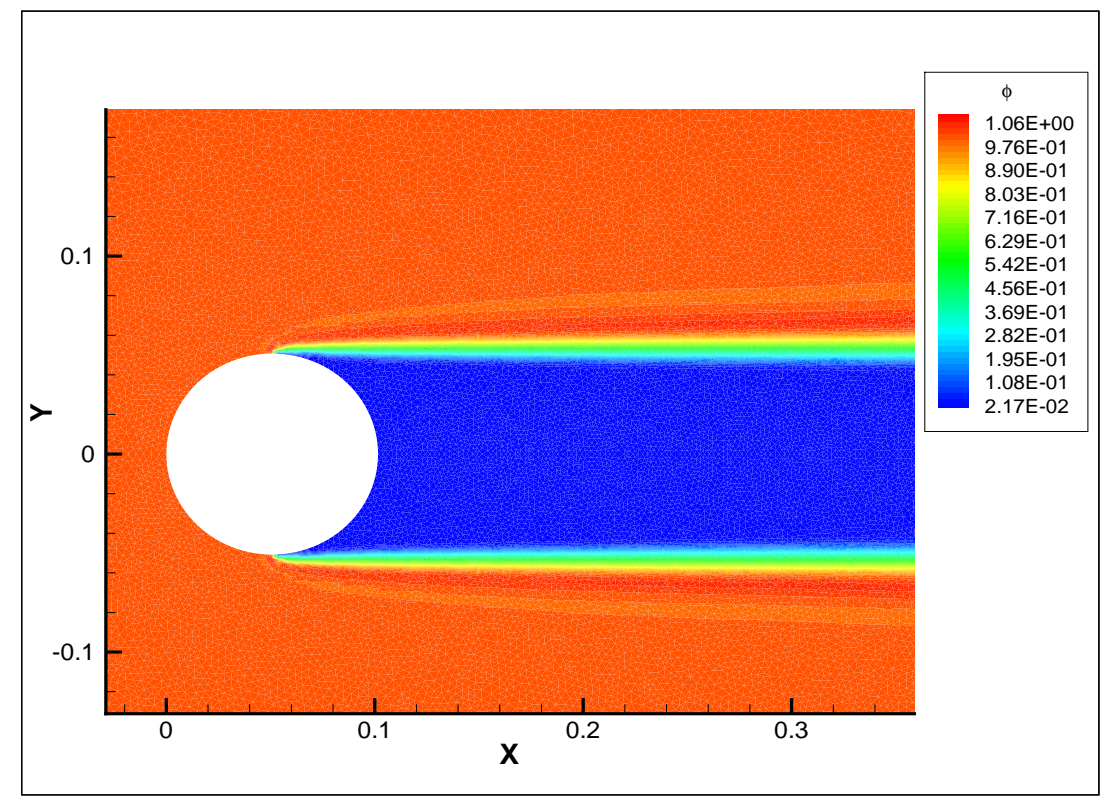

Figura 6.43: Transporte da propriedade $\phi$ na malha $m 5,26866$ vértices: reconstrução cúbica.

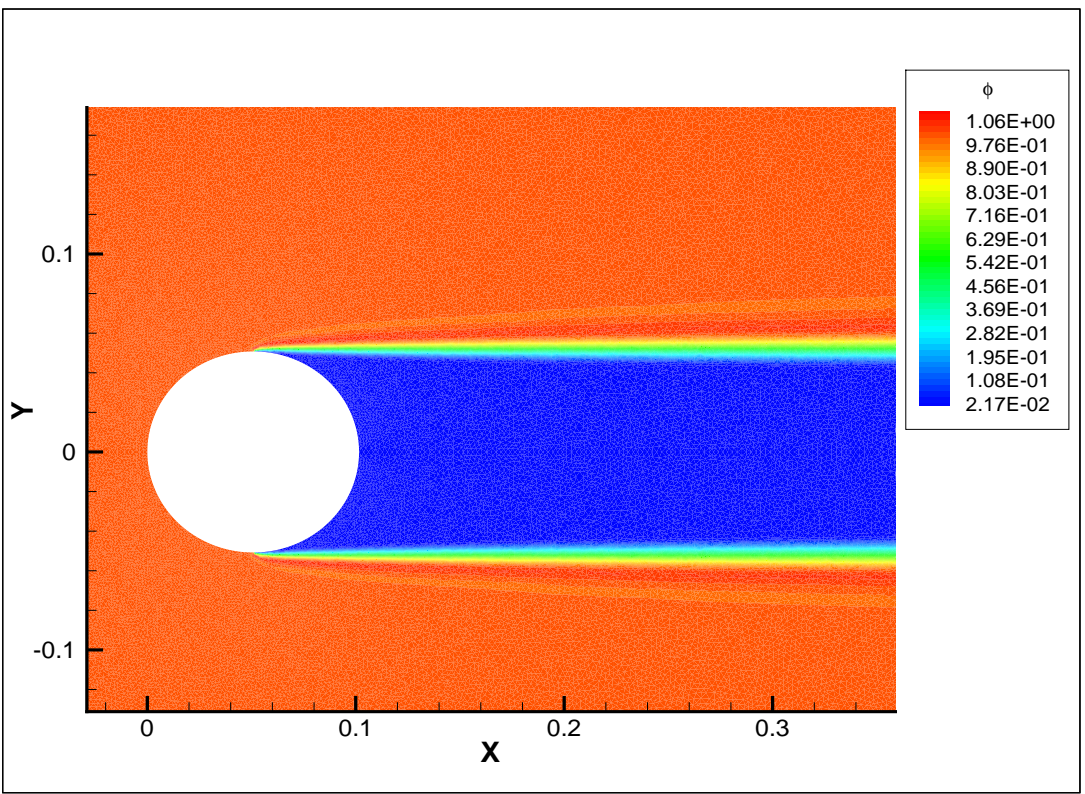

Figura 6.44: Transporte da propriedade $\phi$ na malha $m 6,76013$ vértices: reconstrução linear. 


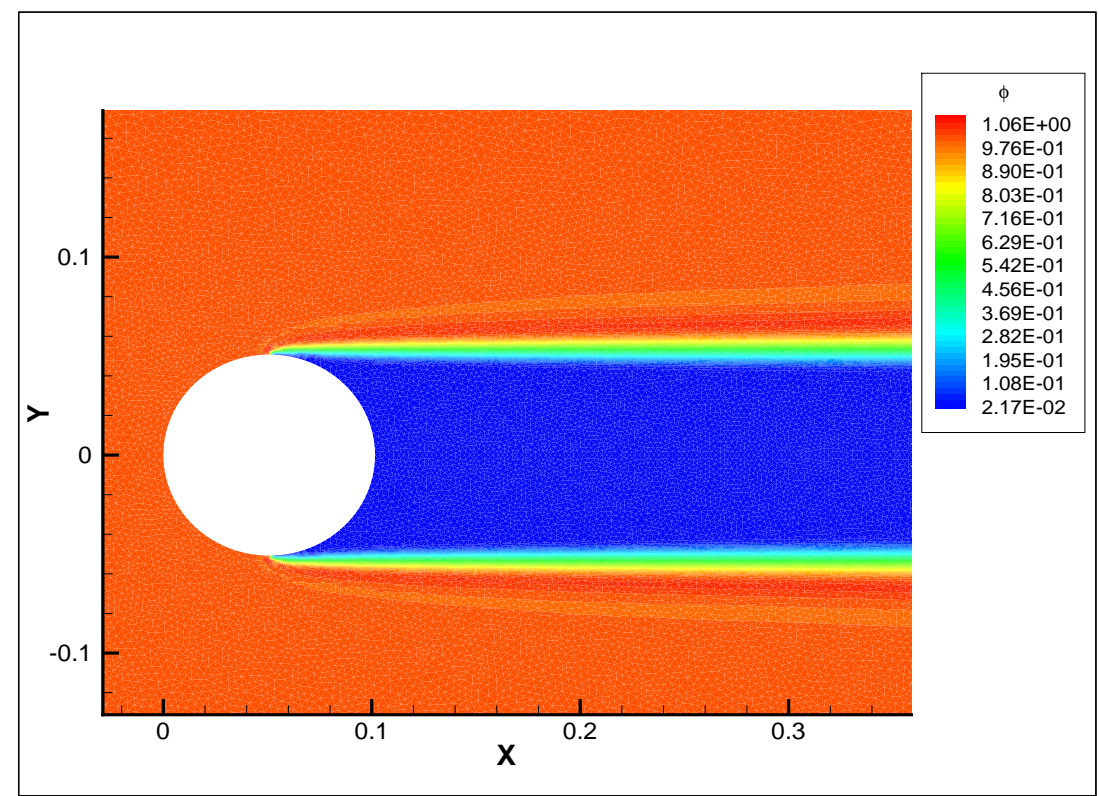

Figura 6.45: Transporte da propriedade $\phi$ na malha $m 6,76013$ vértices: reconstrução quadrática.

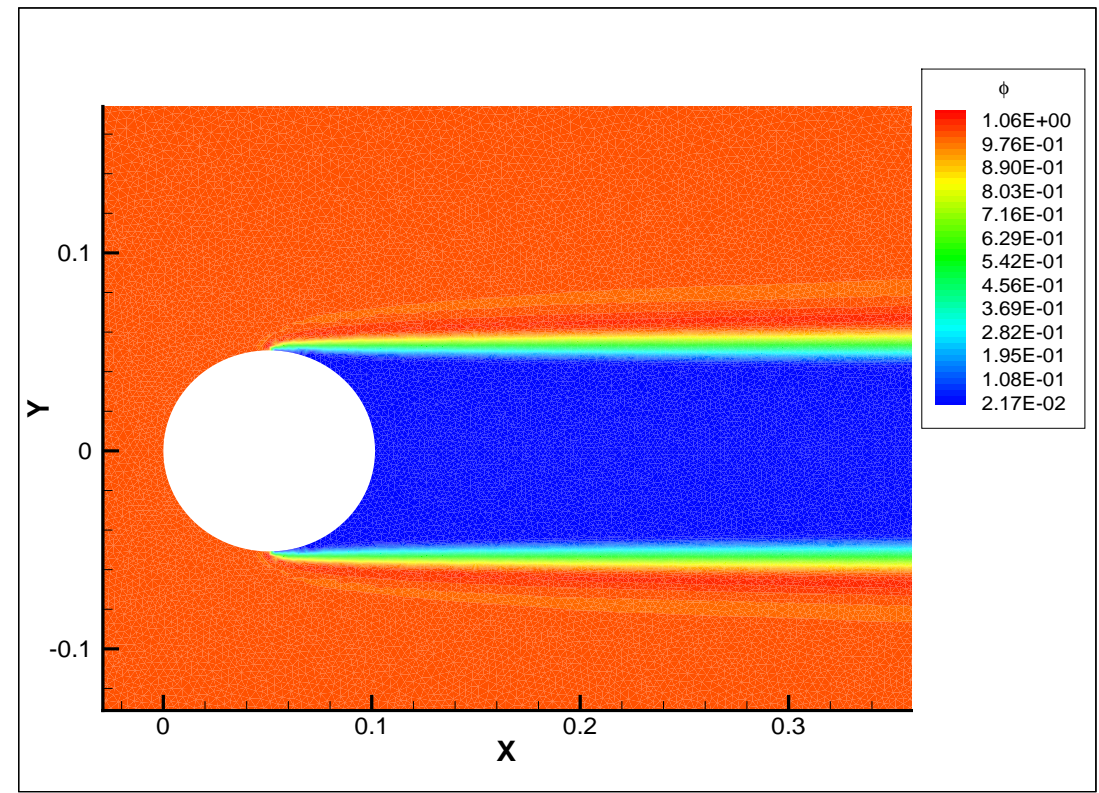

Figura 6.46: Transporte da propriedade $\phi$ na malha m6, 76013 vértices: reconstrução cúbica. 
A Figura 6.47 mostra que os valores de $\phi$ no cilindro não recebem influência dos valores de $\phi$ da região de sombra pois o campo de velocidade para este teste é constante. O mesmo ocorreu para as demais ordens de reconstrução e malhas. Já na reconstrução cúbica, os valores de $\phi$ no contorno sofrem uma pertubação, Figuras 6.34, 6.37, 6.40, 6.43 e 6.46 devido a redução da ordem do polinômio de reconstrução para contorno com condição de Neumann, como foi comentado na seção 5.1.2. A Figura 6.48 mostra como ficam os valores de $\phi$ no cilindro, observando que para a região de sombra continua igual a zero.

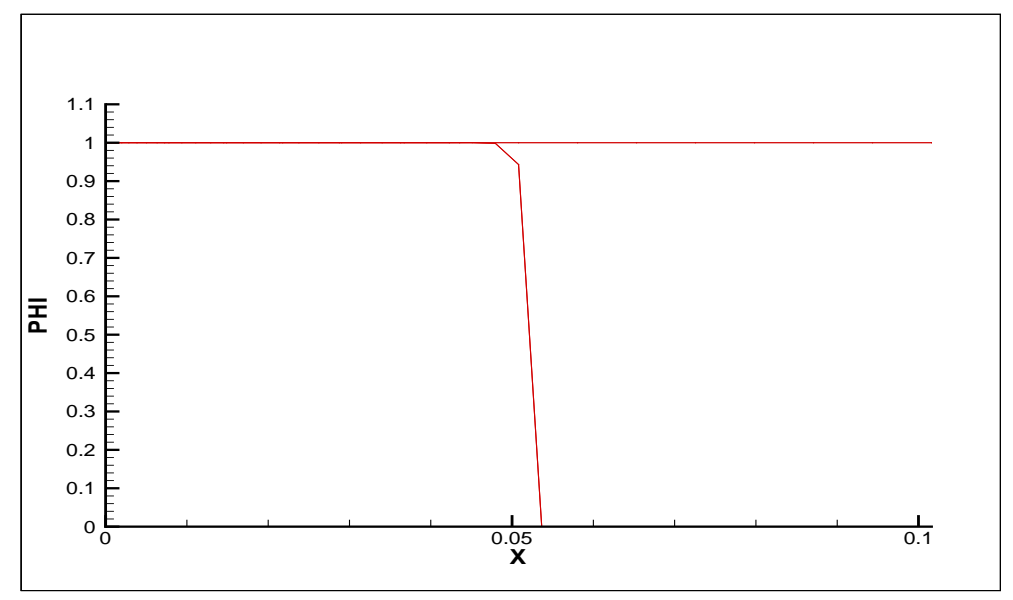

Figura 6.47: Valores da propriedade $\phi$ no cilindro: malha m2 - 13664 vértices, reconstrução linear.

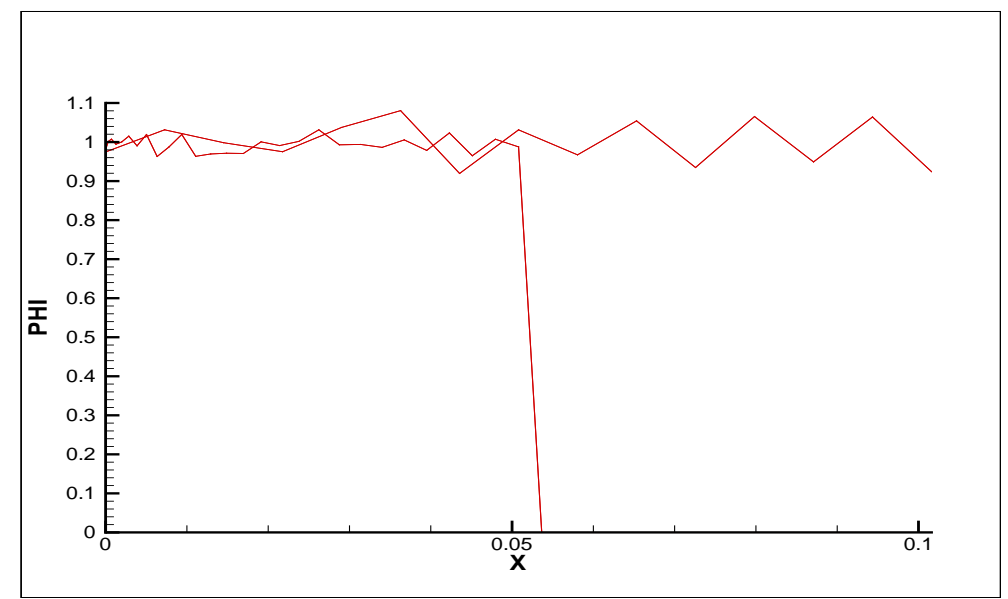

Figura 6.48: Valores da propriedade $\phi$ no cilindro: malha m2 - 13664 vértices, reconstrução cúbica. 
Através das tabelas e gráficos obtidos, observa-se que é possível refinar a malha na região de sombra para a redução da difusão numérica, com ganho no tempo de execução por trabalhar com menos vértices.

Outra observação que se pode perceber é que quanto maior a ordem, mesmo com uma malha mais grossa se consegue uma boa redução da difusão numérica, como pode ser visto com maiores detalhes nos gráficos dos perfis, na próxima seção.

\subsubsection{Estudo do efeito da difusão numérica}

Para o estudo do efeito da difusão numérica foram feitos cortes de perfis nos gráficos dos resultados obtidos na seção anterior. Os cortes para a análise dos perfis são: $p_{1}$ em $\mathrm{x}=0.0, p_{2}$ em $\mathrm{x}=\mathrm{D} / 2$ (passando pelo centro do cilindro), $p_{3}$ em $\mathrm{x}=\mathrm{D}$ (extremidade do diâmetro do cilindro) e $p_{4}$ passando por $\mathrm{x}=0.2$, como mostra a Figura 6.49.

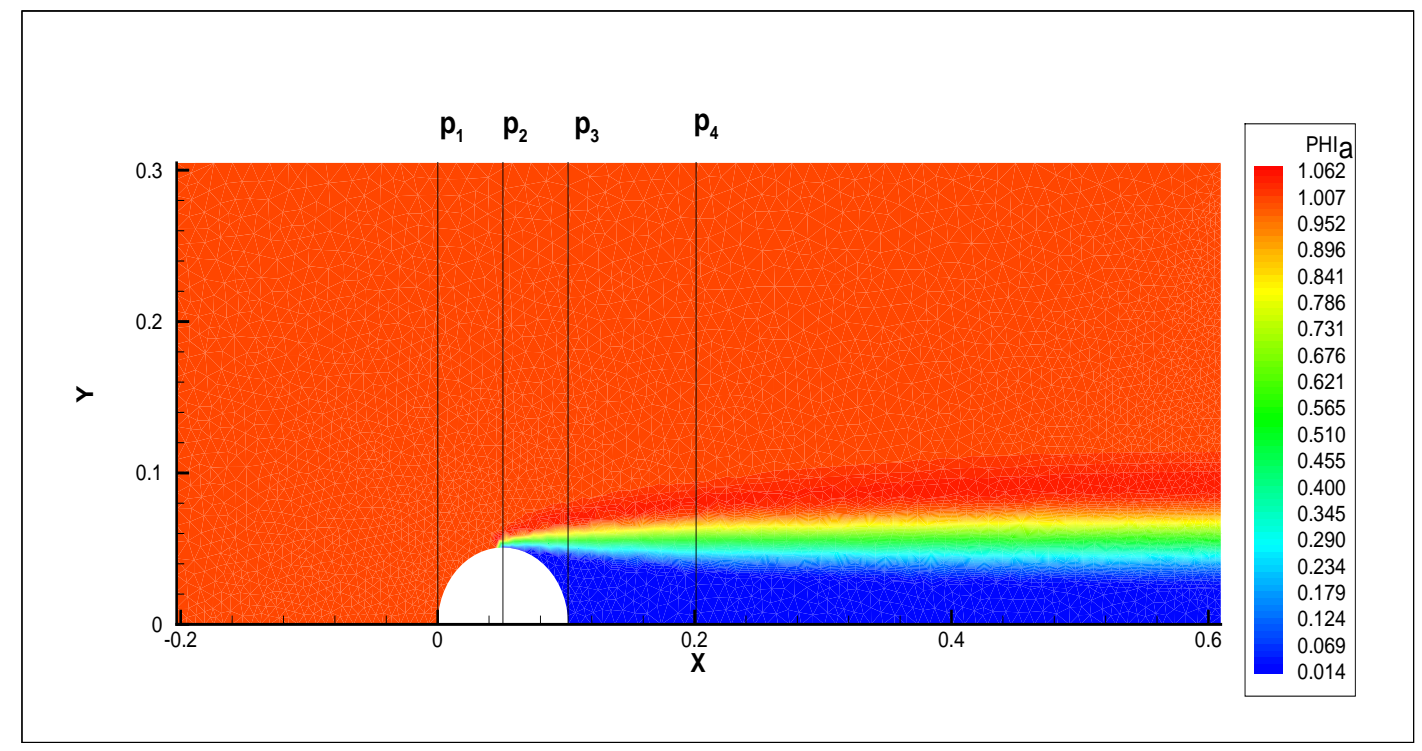

Figura 6.49: Perfis a serem analisados nas malhas $m 1-m 6: p_{1}$ em $\mathrm{x}=0.0, p_{2}$ em $\mathrm{x}=\mathrm{D} / 2$ (centro do cilindro), $p_{3} \mathrm{em} \mathrm{x}=\mathrm{D}$ (extremidade do diâmetro do cilindro) e $p_{4} \mathrm{em} \mathrm{x}=0.2$.

A análise será feita no comportamento da largura da banda à medida que refina-se a malha e também do overshoot resultante da descontinuidade. 


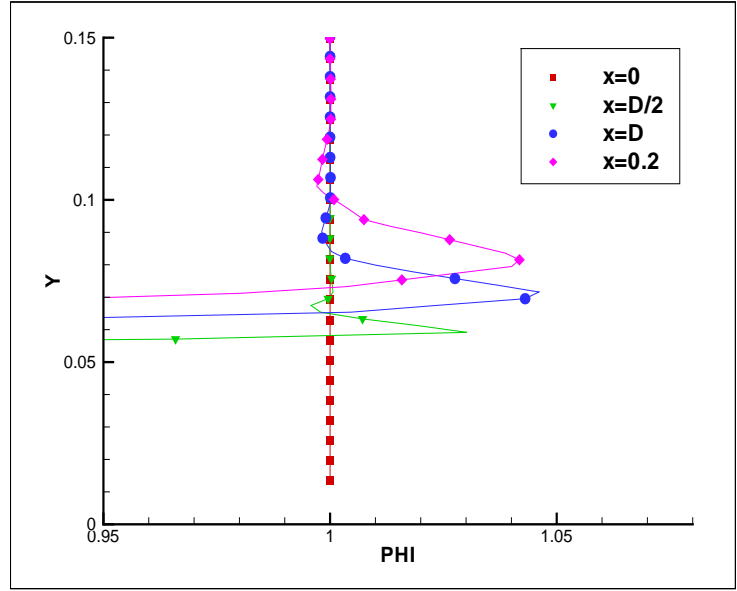

(a)

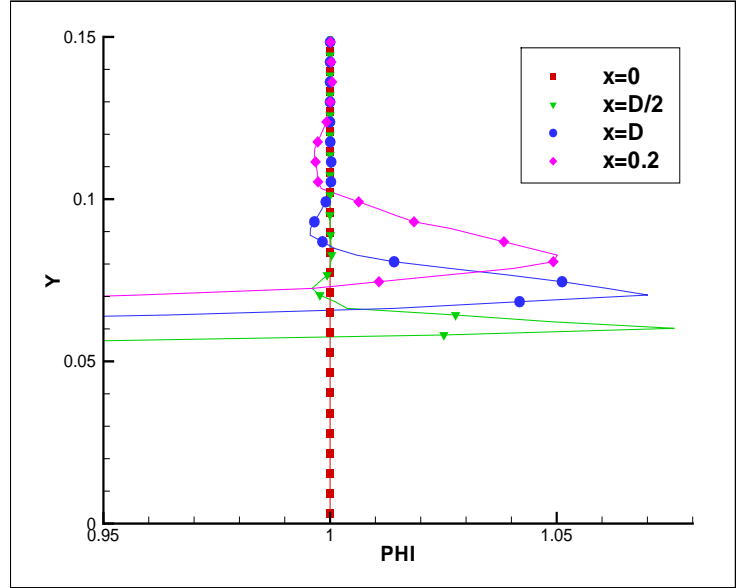

(b)

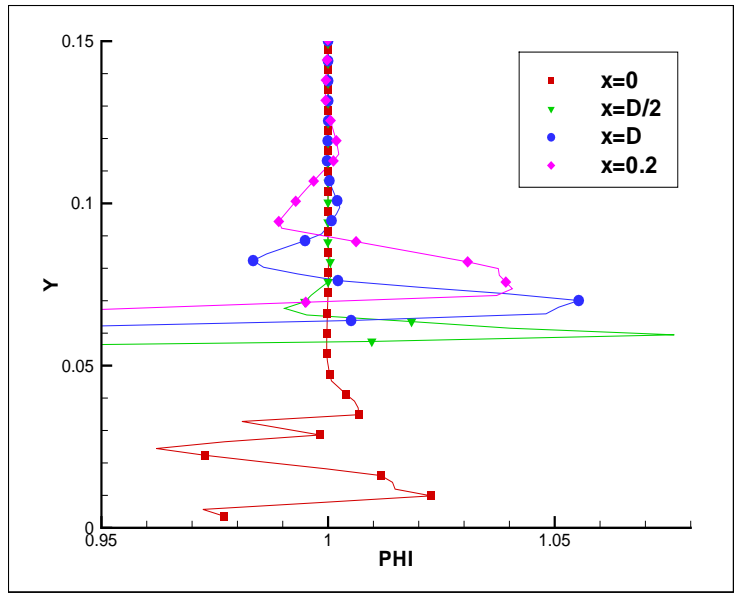

(c)

Figura 6.50: Análise da difusão numérica na malha $m 1$, perfis $p_{1}, p_{2}, p_{3}$ e $p_{4}$, para: (a) reconstrução linear; (b) reconstrução quadrática; (c) reconstrução cúbica. 


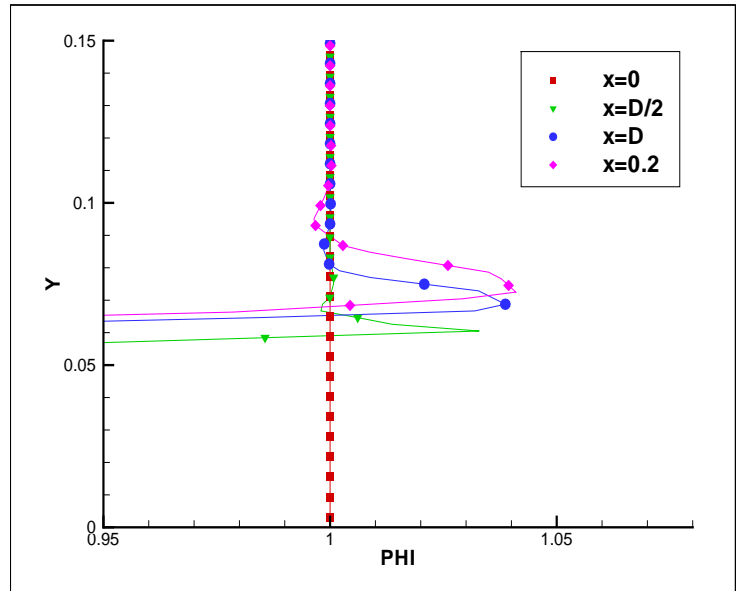

(a)

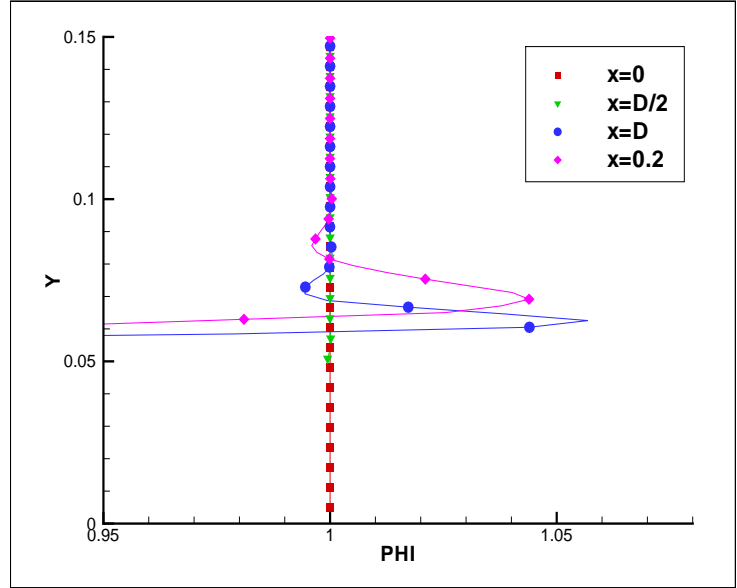

(b)

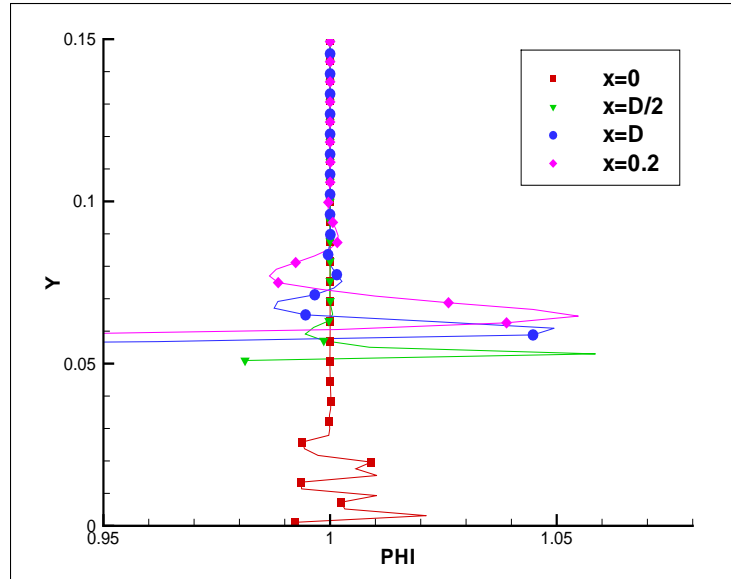

(c)

Figura 6.51: Análise da difusão numérica na malha m2, perfis $p_{1}, p_{2}, p_{3}$ e $p_{4}$, para: (a) reconstrução linear; (b) reconstrução quadrática; (c) reconstrução cúbica. 


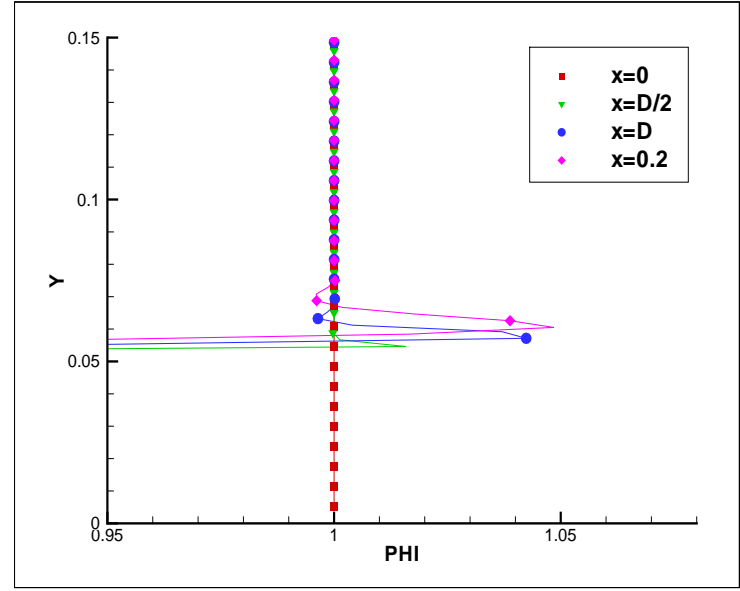

(a)

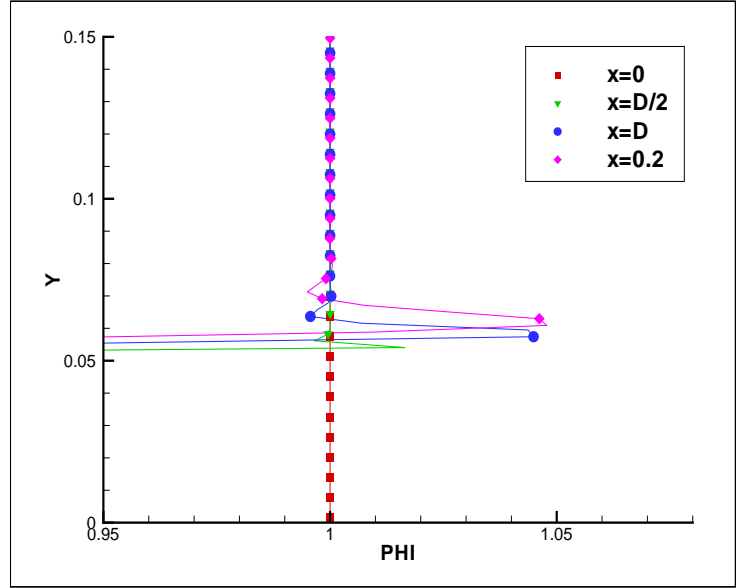

(b)

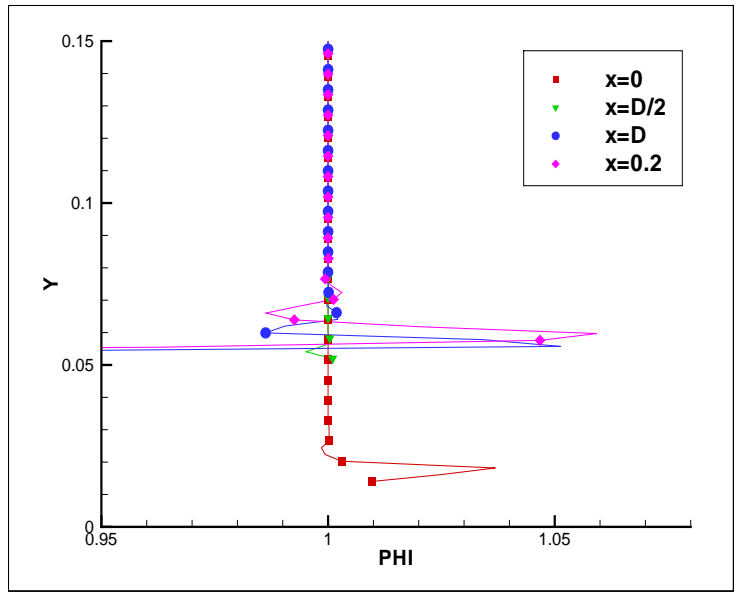

(c)

Figura 6.52: Análise da difusão numérica na malha $m 3$, perfis $p_{1}, p_{2}, p_{3}$ e $p_{4}$, para: (a) reconstrução linear; (b) reconstrução quadrática; (c) reconstrução cúbica. 


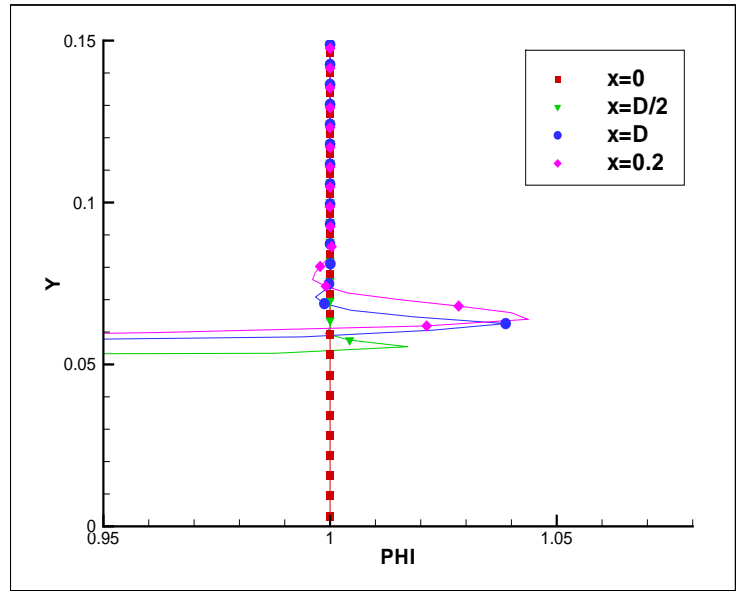

(a)

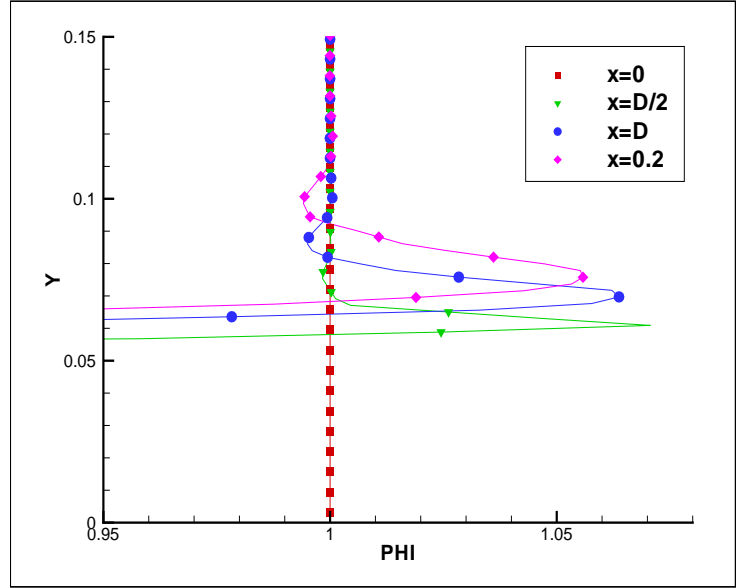

(b)

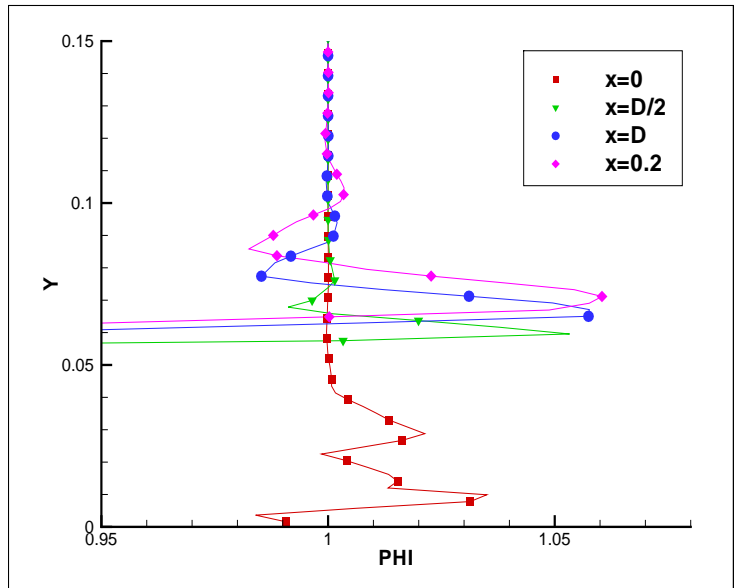

(c)

Figura 6.53: Análise da difusão numérica na malha $m 4$, perfis $p_{1}, p_{2}, p_{3}$ e $p_{4}$, para: (a) reconstrução linear; (b) reconstrução quadrática; (c) reconstrução cúbica. 


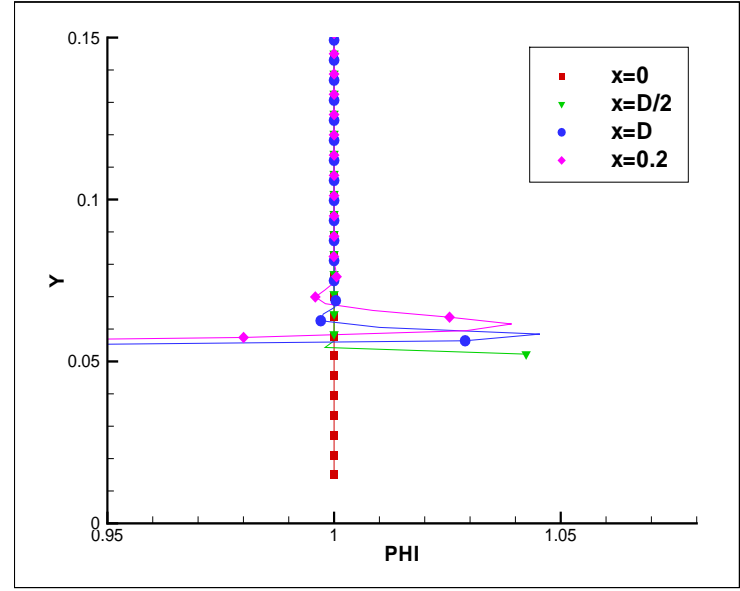

(a)

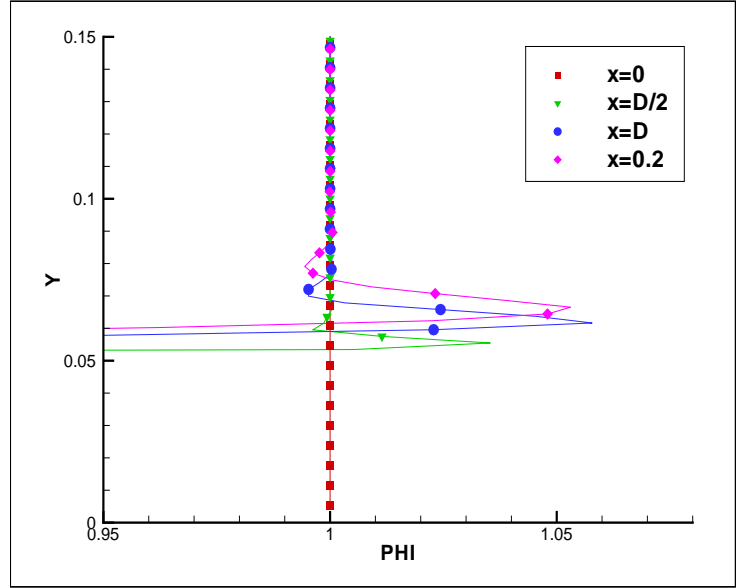

(b)

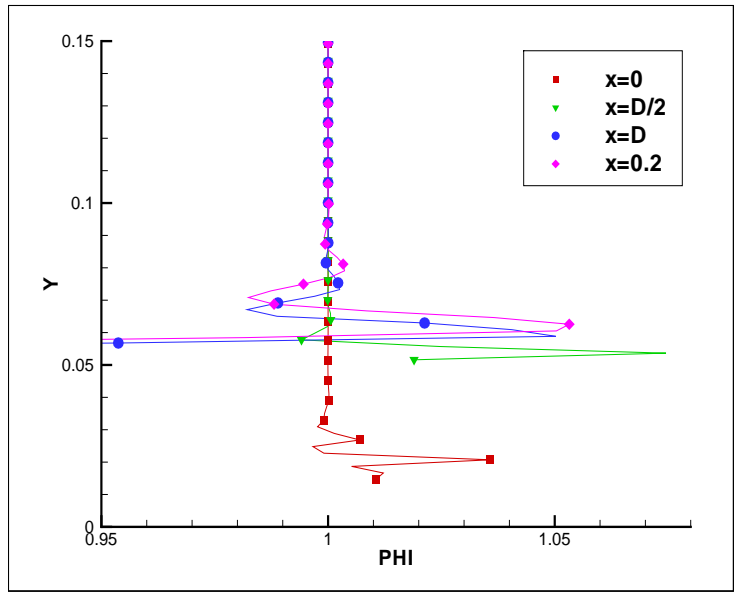

(c)

Figura 6.54: Análise da difusão numérica na malha $m 5$, perfis $p_{1}, p_{2}, p_{3}$ e $p_{4}$, para: (a) reconstrução linear; (b) reconstrução quadrática; (c) reconstrução cúbica. 


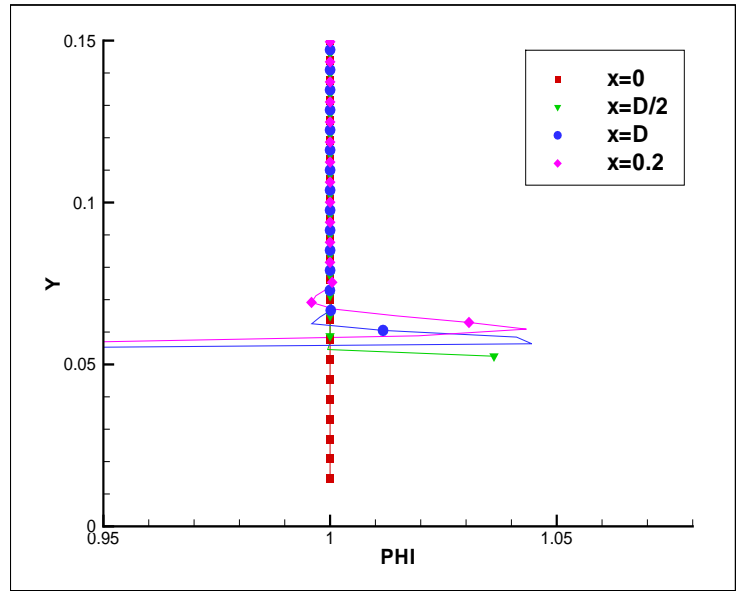

(a)

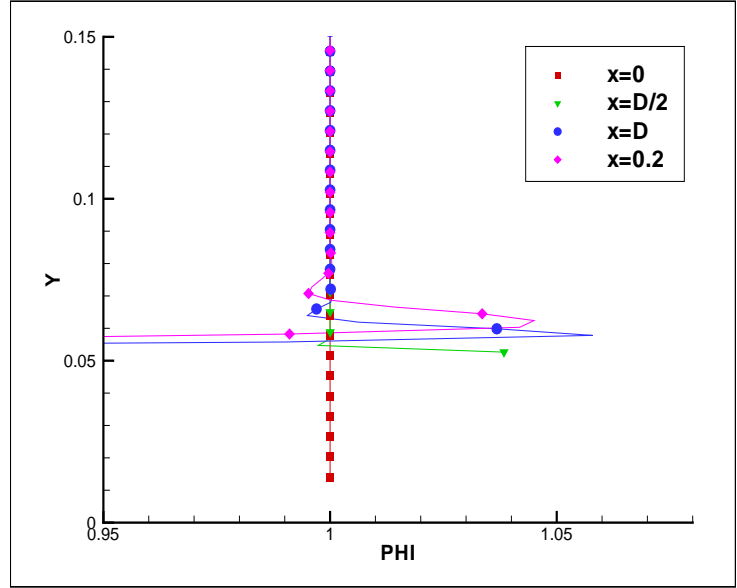

(b)

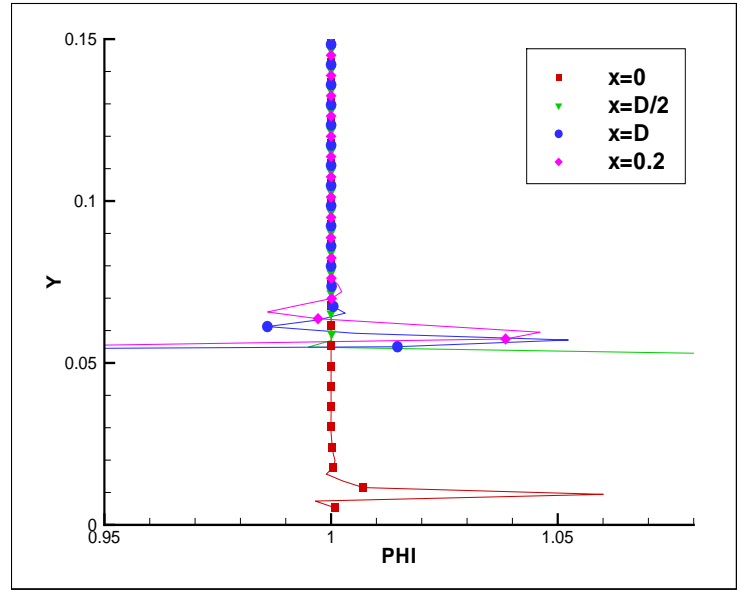

(c)

Figura 6.55: Análise da difusão numérica na malha $m 6$, perfis $p_{1}, p_{2}, p_{3}$ e $p_{4}$, para: (a) reconstrução linear; (b) reconstrução quadrática; (c) reconstrução cúbica. 
A Figura 6.53 apresenta os perfis $p_{1}-p_{4}$ para as ordens de reconstrução linear, quadrática e cúbica respectivamente, com refinamento da região de sombra em relação à Figura 6.50 e observa-se uma redução da banda em todas as ordens bem como do overshoot. Em análise análoga, da Figura 6.54 com a Figura 6.51 obervou-se o mesmo comportamento. Já na análise da Figura 6.55 com Figura 6.52, que são as malhas mais refinadas, observou-se uma redução do overshoot na ordem de reconstrução 2, porém um aumento no overshoot para a ordem 3 e 4 . A largura da banda, que mostra o comportamento da difusão numérica se reduz à medida que se refina a malha num geral, mais ainda ao refinar a região de sombra.

Para mostrar a influência da ordem na análise da difusão numérica, fez-se a Figura 6.56, onde é possível ver claramente a redução da difusão numérica para o perfil $p_{4}$ pela largura da banda e também o comportamento do overshoot. E para mostrar a influência da malha na difusão numérica, fez-se a Figura 6.57, onde pode ser visto a redução da banda à medida que se refina as malhas com um aumento no overshoot ao refinar as malhas. 


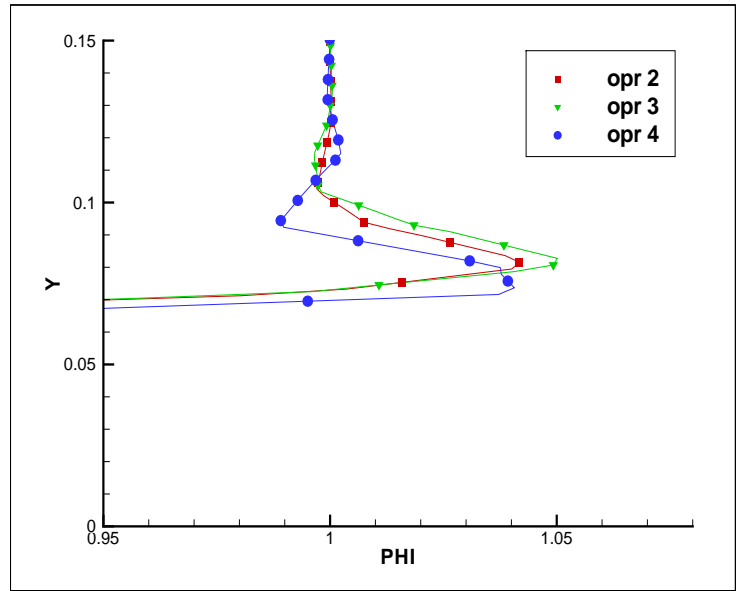

(a)

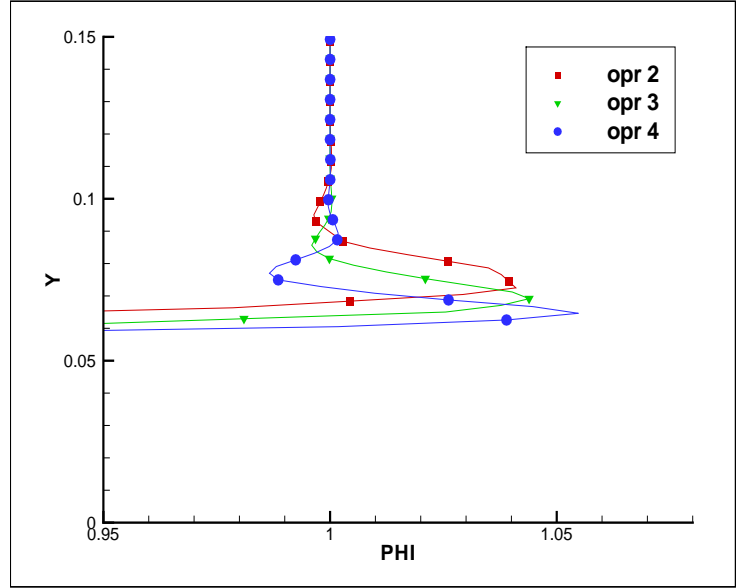

(b)

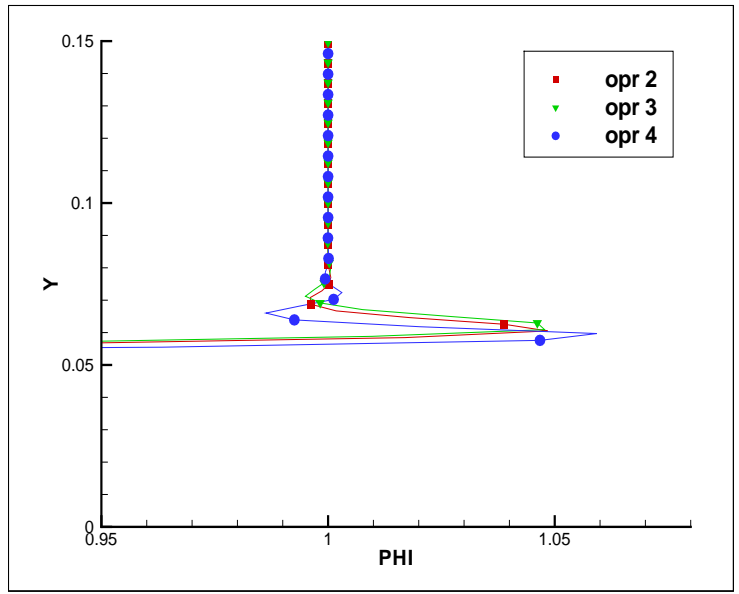

(c)

Figura 6.56: Análise da difusão numérica, perfil $p_{4}$, para as malhas: (a) m1; (b) m2; (c) malha $m 3$. 


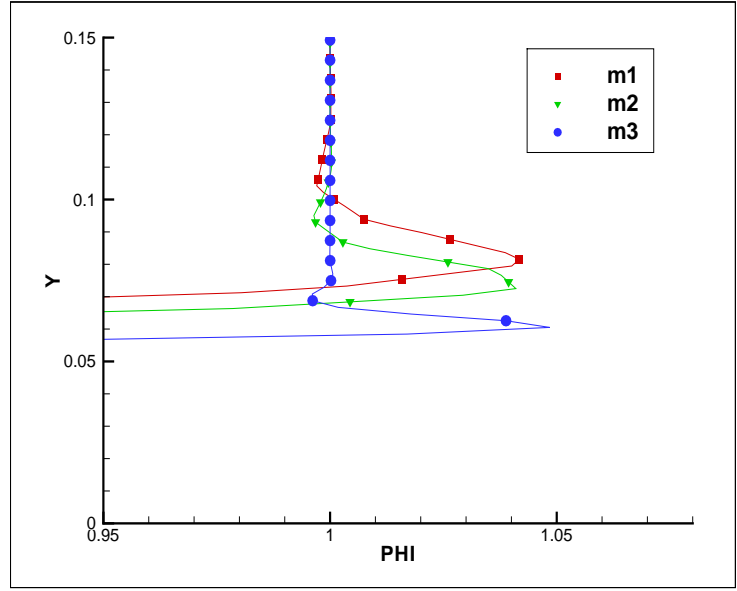

(a)

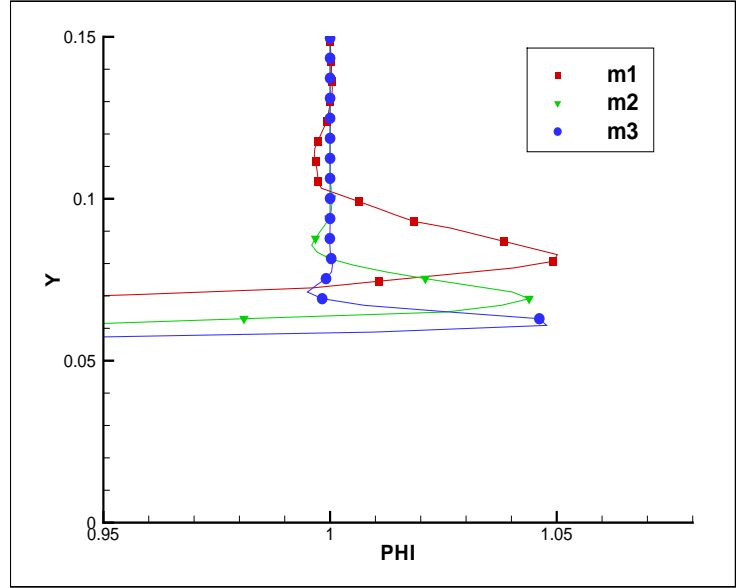

(b)

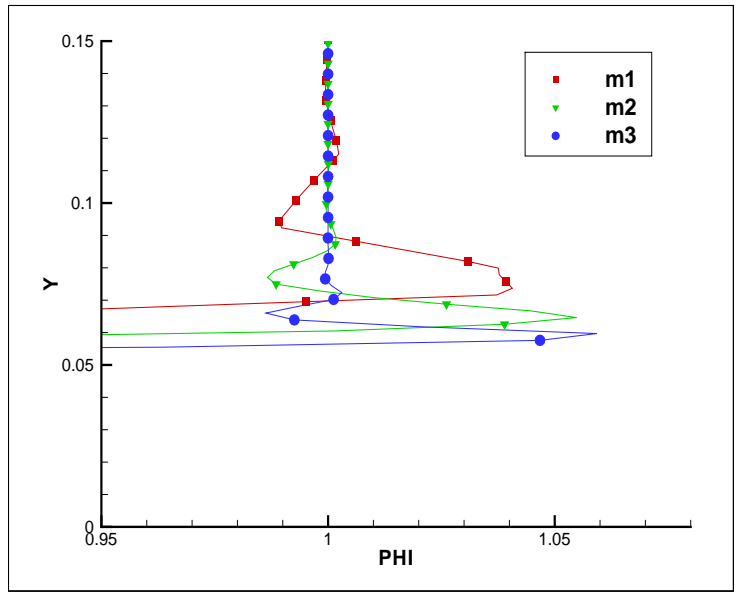

(c)

Figura 6.57: Análise da difusão numérica, perfil $p_{4}$, para as malhas m1, m2 e m3: (a) reconstrução linear; (b) reconstrução quadrática; (c) reconstrução cúbica. 
Com os testes realizados, mostrou-se que o método de reconstrução mínimos quadrados com alta ordem com é uma ferramenta que pode ser usada na resolução de problemas com descontinuidades como é a região de sombra atrás de um cilindro. À medida que se refina a malha ou aumenta-se a ordem do polinômio de reconstrução tem-se uma redução da difusão numérica. 


\section{CAPÍtulo 7}

\section{Conclusão}

O objetivo principal deste trabalho foi apresentar o esquema numérico reconstrução mínimos quadrados com alta ordem, aplicando-o em várias equações-modelo, mostrando que mesmo pode ser utilizado no cálculo do transporte de gotículas em uma formulação euleriana.

Assim, o método de alta ordem apresentado por Gooch e colaboradores [22, 23, 24, $25,26,52,70]$, que usa a reconstrução mínimos quadrados para reconstruir uma função em malhas não-estruturadas com formulação cell-vertex, permite trabalhar com células triangulares conectadas a células quadrangulares ou com outra forma polinomial em 2D. O mesmo podendo ser estendido para 3D, possibilitando, de forma prática trabalhar com geometrias complexas.

A reconstrução pode ser feita tanto de uma função conhecida ou apartir dos seus valores nodais, através da média destes valores no volume de controle.

Nos testes realizados no transporte da propriedade $\phi$, capítulo 6, concluiu-se que para a condição de contorno de Neumann observa-se a re-impingência, o mesmo ocorre quando se define a condição de contorno a partir do ponto de descolamento.

Quando a condição de contorno é definida a partir da componente da normal, $n_{x}$, não se observa a re-impingência. Então fez-se um estudo do efeito da difusão numérica na descontinuidade vertical presente e, à medida que se refina a malha ou aumenta a ordem do polinômio de reconstrução há uma redução da largura da banda ou do overshoot, Figura 7.1. Outro detalhe, é o refinamento na região atrás do cilindro, que permite reduzir a difusão numérica com um ganho de tempo computacional. 


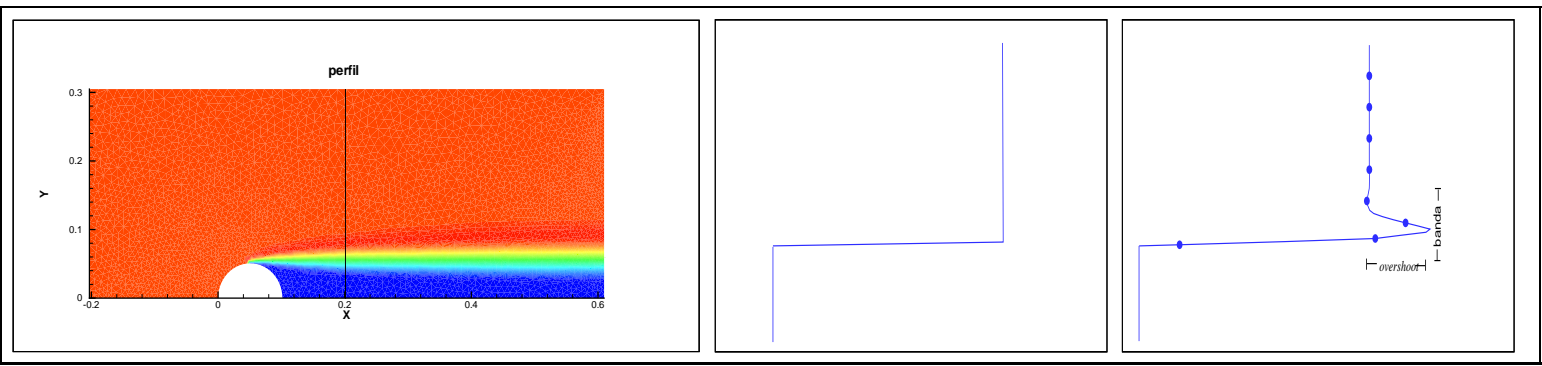

(a)

(b)

(c)

Figura 7.1: Estudo da difusão numérica: (a) traçado do perfil; (b) perfil "ideal"; (c) perfil obtido.

Com base em todos estes testes com a análise do transporte da propriedade $\phi$, concluise que o mesmo pode se estender para o estudo da obtenção da fração de água líquida $\alpha$ para a obtenção de $\beta$, atendendo o objetivo central deste trabalho que era avaliar o potencial do uso do método de reconstrução mínimos quadrados com alta ordem na resolução de problemas de advecção-difusão 2D que teve como motivação a resolução do transporte de gotículas em formulação Euleriana na determinação de $\alpha$.

Esquemas de alta ordem em malhas não-estruturadas é algo relativamente recente. Assim ainda há muito a pesquisar sobre as vantagens práticas da discretização de alta ordem em malhas não-estruturadas.

Como trabalho futuro, pode-se complementar a estrutura de dados permitindo ter uma boa conectividade entre os dados da malha utilizados no pré-processamento; buscar outras formas de resolver o sistema linear com restrição no contorno, buscando solucionar o problema apresentado. Ainda, fazer um estudo mais aprofundado do que se tem nos artigos mais recentes de Gooch et al. [27, 44, 45], uma vez que esta técnica está em constante aperfeiçoamento.

Na parte da formulação euleriana, obter a fração de água líquida $\alpha$ para fazer um estudo com o coeficiente de coleta $\beta$ no aerofólio e em geometrias de aerofólio com multielementos. 


\section{Esquema de separação do vetor fluxo}

Com o intuito de estabilizar a aproximação da solução de uma equação hiperbólica por um método numérico para o termo convectivo, a adição de um termo suavizador pode ser requisitada para reduzir as oscilações que podem surgir na vizinhança de grandes variações do gradiente. O método pode ser estável se a equação governante incluir um termo difusivo. Para evitar o uso de termos suavizadores, uma alternativa é adotar o esquema de separação do vetor fluxo para formular o termo convectivo.

Para apresentar o esquema de separação do vetor fluxo serão adotadas as equações de Euler axissimétricas e em duas dimensões (2D) na forma vetorial (A.1) que descrevem um escoamento invíscido e isotérmico (forma conservativa).

$$
\frac{\partial U}{\partial t}+\left(\frac{\partial F}{\partial x}+\frac{\partial G}{\partial y}\right)+\alpha H=0
$$

onde,

$U$ é o vetor estado das variáveis dependentes (conservação das variáveis).

$F$ e $G$ são os fluxos conservativos na direção x e y, respectivamente. F e G são funções do vetor estado U.

$$
\mathrm{U}=\left(\begin{array}{c}
\rho \\
\rho u \\
\rho v \\
\rho e_{t}
\end{array}\right), \mathrm{F}=\left(\begin{array}{c}
\rho u \\
\rho u^{2}+p \\
\rho u v \\
\left(\rho e_{t}+p\right) u
\end{array}\right), \mathrm{G}=\left(\begin{array}{c}
\rho v \\
\rho u v \\
\rho v^{2}+p \\
\left(\rho e_{t}+p\right) v
\end{array}\right) \text { e } \mathrm{H}=\frac{1}{y}\left(\begin{array}{c}
\rho u \\
\rho u v \\
\rho v^{2} \\
\left(\rho e_{t}+p\right) v
\end{array}\right)
$$


sendo $\alpha=\left\{\begin{array}{l}0, \text { para um escoamento } 2 \mathrm{D} . \\ 1, \text { para um escoamento } 2 \mathrm{D} \text { axissimétrico. }\end{array}\right.$

$\rho$ é a massa específica do fluido.

$u$ e $v$ são as componentes da velocidade na direção x e y, respectivamente.

$p$ é a pressão estática.

$e_{t}$ é a energia total específica,

$$
e_{t}=e+1 / 2\left(u^{2}+v^{2}\right)
$$

$e$ é a energia interna padrão.

\section{Separação do vetor fluxo para os triângulos internos}

A equação (A.1) é integrada em um elemento triangular $a b c$ para $\alpha=1$, da Figura A.1 para obter

$$
\int_{a b c}\left(\frac{\partial U}{\partial t}\right) d x d y=\underbrace{-\int_{a b c}\left(\frac{\partial F}{\partial x}+\frac{\partial G}{\partial y}+H\right) d x d y}_{R H S}
$$

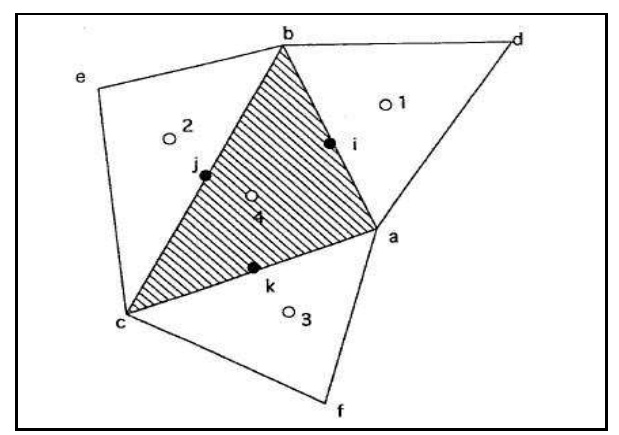

Figura A.1: Elemento triangular $a b c$ e seus elementos adjacentes [33].

Aplicando o Teorema de Green para $R H S$ e empregando um esquema centrado explícito na célula para aproximar (A.2), temos:

$$
\begin{gathered}
\left(\frac{U_{4}^{n+1}-U_{4}^{n}}{\Delta t}\right) A_{a b c}=-\left[\left(F_{i} \Delta y_{a b}+F_{j} \Delta y_{b c}+F_{k} \Delta y_{c a}\right)-\left(G_{i} \Delta x_{a b}+G_{j} \Delta x_{b c}+\right.\right. \\
\left.\left.+G_{k} \Delta x_{c a}\right)+H_{4} A_{a b c}\right]
\end{gathered}
$$

A Figura A.2 esquematiza a evolução dos fluxos F e G para o ponto $i$ localizado no ponto médio da face $a b$. Cada fluxo é dividido na parte positiva e negativa associada ao autovalor correspondente, essência do método de separação do vetor fluxo. 


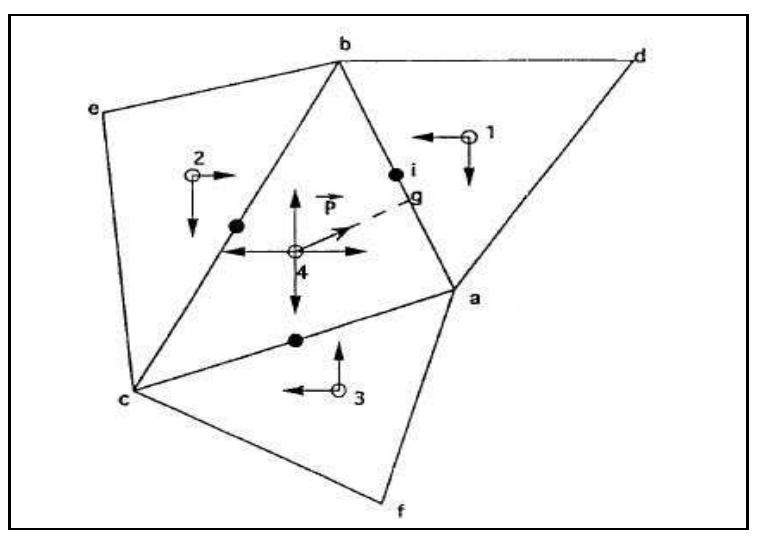

Figura A.2: Ilustração dos sinais de propagação [33].

No esquema volumes finitos a fomulação é aplicada diretamente no espaço físico, assim é preciso obter os fluxos associados a esse espaço. Hoffmann [33] apresenta os cálculos para determinar os autovalores da matriz jacobiana $A=\frac{\partial F}{\partial U}: \lambda_{A 1}=u, \lambda_{A 2}=u, \lambda_{A 3}=u+a$ e $\lambda_{A 4}=u-a$, onde $u$ é a componente da velocidade na direção x e $a$ é a velocidade do som. Analogamente para $B=\frac{\partial G}{\partial U}$ tem-se os seguintes autovalores: $\lambda_{B 1}=v, \lambda_{B 2}=v$, $\lambda_{B 3}=v+a$ e $\lambda_{B 4}=v-a$ usados para efetuar a separação dos fluxos.

Uma vez determinados os autovalores das matrizes jacobianas A e B, elas podem ser separadas em

$$
\begin{aligned}
& A=A^{+}+A^{-} \\
& B=B^{+}+B^{-}
\end{aligned}
$$

onde

$$
\begin{aligned}
& A^{+}=X_{A} D_{A}^{+} X_{A}^{-1} \\
& A^{-}=X_{A} D_{A}^{-} X_{A}^{-1} \\
& B^{+}=X_{B} D_{B}^{+} X_{B}^{-1} \\
& B^{-}=X_{B} D_{B}^{-} X_{B}^{-1}
\end{aligned}
$$

A matriz $D_{A}^{+}$é a matriz diagonal cujos elementos são os autovalores positivos de A e $D_{A}^{-}$é a matriz diagonal cujos elementos são os autovalores negativos de A. A analogia é extensível para $D_{B}^{+}$e $D_{B}^{-}$. 
As matrizes $X_{A}$ e $X_{B}$, (A.4) e (A.5), são as matrizes dos autovetores de A e B, respectivamente e $X_{A}^{-1}$ e $X_{B}^{-1}$ denota as suas inversas.

$$
\begin{gathered}
X_{A}=\left[\begin{array}{cccc}
0 & 1 & 1 & 1 \\
0 & u & u+a & u-a \\
1 & 0 & v & v \\
v & \frac{1}{2}\left(u^{2}-v^{2}\right) & \frac{u^{2}+v^{2}}{2}+\frac{a^{2}}{\gamma-1}+a u & \frac{u^{2}+v^{2}}{2}+\frac{a^{2}}{\gamma-1}-a u
\end{array}\right] \\
X_{B}=\left[\begin{array}{cccc}
0 & 1 & u+a & 1 \\
0 & u & v & u-a \\
1 & 0 & \frac{u^{2}+v^{2}}{2}+\frac{a^{2}}{\gamma-1}+a u & \frac{u^{2}+v^{2}}{2}+\frac{a^{2}}{\gamma-1}-a u
\end{array}\right]
\end{gathered}
$$

E o processo de separação do vetor $\mathrm{F}$ é feito através da análise dos autovalores, como segue:

i) Se $u>a$ então, todos os autovalores são positivos e portanto

$$
F^{+}=F
$$

e

$$
F^{-}=0
$$

ii) Se $0<u<a$, um dos autovalores, $\lambda_{4}$, é negativo e

$$
F^{-}=(u-a)\left(\frac{\rho}{2 \gamma}\right)\left[\begin{array}{c}
1 \\
u-a \\
v \\
-a u+\frac{1}{2} q^{2}+\frac{a^{2}}{\gamma-1}
\end{array}\right]
$$

onde

$$
q^{2}=u^{2}+v^{2}
$$

e

$$
F^{+}=F-F^{-} .
$$


iii) Se $-a<u<0$, um dos autovalores, $\lambda_{3}$, é positivo e

$$
F^{+}=(u-a)\left(\frac{\rho}{2 \gamma}\right)\left[\begin{array}{c}
1 \\
u+a \\
v \\
a u+\frac{1}{2} q^{2}+\frac{a^{2}}{\gamma-1}
\end{array}\right]
$$

$\mathrm{e}$

$$
F^{-}=F-F^{+}
$$

iv) Se $u \leq-a$ então, todos os autovalores são negativos, portanto

$$
F^{+}=0
$$

e

$$
F^{-}=F
$$

Da forma similar os fluxos $G^{+}$e $G^{-}$associados às quatro possíveis combinações dos autovalores de $B$ podem se obtidos. Então os fluxos podem ser decompostos em suas partes negativa e positiva com a forma exata. Por exemplo, para o ponto de controle 4, que é ilustrado na Figura A.2

$$
F_{i}=F_{4}^{+}+F_{1}^{-}
$$

$\mathrm{e}$

$$
G_{i}=G_{4}^{+}+G_{1}^{-}
$$

O algoritmo apresentado é simplesmente baseado na inspeção gráfica da Figura A.2 e esta conclusão é usada para desenvolver o esquema numérico usado para calcular $U_{4}^{n+1}$ :

1. Determinar o vetor $\vec{P}$ do ponto de controle 4 perpendicular à face $a b$, obtendo o ponto $g$, Figura A.3.

$$
\vec{P}=\left(x_{g}-x_{4}\right) \vec{i}+\left(y_{g}-y_{4}\right) \vec{j}=\left[\left(y_{4}-n-m x_{4}\right) /\left(m^{2}+1\right)\right](m \vec{i}-\vec{j})=P_{4 x} \vec{i}+P_{4 y} \vec{j},
$$

onde

$$
m=\frac{y_{b}-y_{a}}{x_{b}-x_{a}} \quad \text { e } \quad n=\frac{x_{b} y_{a}-y_{b} x_{a}}{x_{b}-x_{a}}
$$


e as coordenadas do ponto de intersecção $g$ são

$$
x_{g}=\left(m y^{4}+x_{e}-m x\right) /\left(m^{2}+1\right) \quad \text { e } y_{g}=\left(m^{2} y^{4}+m x^{4}+n\right) /\left(m^{2}+1\right) .
$$

Se $x_{a}=x_{b}$

$$
P_{4}=\left(x_{a}-x_{4}\right) \vec{i}=P_{4 x} \vec{i}+P_{4 y} \vec{j}
$$

2. Determinar F e G para os pontos médios das faces.

Para o ponto médio $i$ da face $a b$, tem-se:

i) Se $P_{4 x} \geq 0$, então o ponto de controle 4 contribui $F_{4}^{+}$para i e o ponto $1, F_{1}^{-}$

$$
F_{i}=F_{4}^{+}+F_{1}^{-}
$$

ii) Se $P_{4 x}<0$,

$$
F_{i}=F_{4}^{-}+F_{1}^{+}
$$

O procedimento é estendido para o vetor fluxo G:

$$
\begin{gathered}
G_{i}=G_{4}^{+}+G_{1}^{-}, \text {para } P_{4 y}>0, \\
F_{i}=G_{4}^{-}+G_{1}^{+}, \text {para } P_{4 y}<0 .
\end{gathered}
$$

O processo é repetido para os demais pontos médios, $j$ e $k$.

3. Usar (A.3) para calcular $U_{4}^{n+1}$.

Para facilitar a análise e implementação das condições de contorno, os pontos de controle são deslocados para os pontos médios da face do contorno, Figura A.3, e a implementação é apresentada em [33] para as diversas condições de contorno em um domínio axissimétrico de um corpo que não apresenta grandes variações nos gradientes em um escoamento supersônico.

\section{Aplicação na equação do transporte de partículas}

Comparando as equações do transporte de partículas (2.9) com as equações de Euler (A.1), na forma vetorial:

$$
\frac{\partial U}{\partial t}=-\left(\frac{\partial F}{\partial x}+\frac{\partial G}{\partial y}\right)+H
$$

onde 


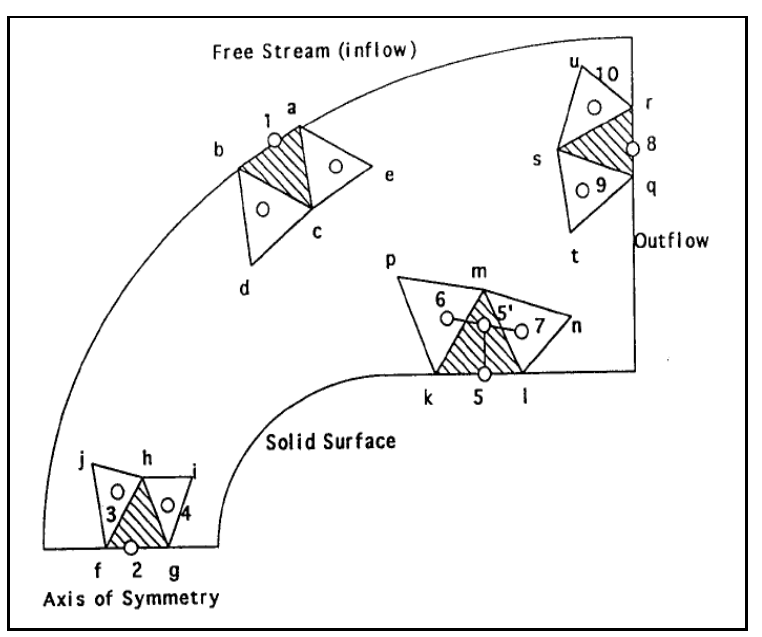

Figura A.3: Esquematização para as condições de contorno [33].

$$
\mathrm{U}=\left(\begin{array}{c}
\alpha \rho \\
\alpha \rho u \\
\alpha \rho v
\end{array}\right), \quad F=\left(\begin{array}{c}
\alpha \rho \\
\alpha \rho u^{2} \\
\alpha \rho u v
\end{array}\right), \quad G=\left(\begin{array}{c}
\alpha \rho v \\
\alpha \rho u v \\
\alpha \rho v^{2}
\end{array}\right) \text { e } \quad H
$$

com,

$\alpha$ : coeficiente de coleta.

$\rho$ : densidade da partícula.

$u$ : componente da velocidade da partícula na direção x.

$v$ : componente da velocidade da partícula na direção y.

$H$ : termo forçante.

A matriz jacobiana $A=\frac{\partial F}{\partial U}$ é dada por

$$
A=\frac{\partial F}{\partial U}=\left(\begin{array}{lll}
\frac{\partial F_{1}}{\partial U_{1}} & \frac{\partial F_{1}}{\partial U_{2}} & \frac{\partial F_{1}}{\partial U_{3}} \\
\frac{\partial F_{2}}{\partial U_{1}} & \frac{\partial F_{2}}{\partial U_{2}} & \frac{\partial F_{2}}{\partial U_{3}} \\
\frac{\partial F_{3}}{\partial U_{1}} & \frac{\partial F_{3}}{\partial U_{2}} & \frac{\partial F_{3}}{\partial U_{3}}
\end{array}\right)
$$

Para determinar os elementos da matriz (A), os vetores $\mathrm{F}$ e $\mathrm{G}$ são expressos em termos das componentes do vetor U através da linearização.

$F_{1}=U_{2}=\alpha \rho u$

$F_{2}=U_{2}^{2} / U_{1}=\frac{\alpha^{2} \rho^{2} u^{2}}{\alpha \rho}=\alpha \rho u^{2}$

$F_{3}=U_{2} * U_{3} / U_{1}=\alpha \rho u v$ 
$G_{1}=U_{3}=\alpha \rho v$

$G_{2}=U_{2} * U_{3} / U_{1}=\alpha \rho u v$

$G_{3}=U_{3}^{2} / U_{1}=\alpha \rho v^{2}$

Assim,

$$
\begin{aligned}
& A=\frac{\partial F}{\partial U}=\left(\begin{array}{ccc}
\frac{\partial U_{2}}{\partial U_{1}} & \frac{\partial U_{2}}{\partial U_{2}} & \frac{\partial U_{2}}{\partial U_{3}} \\
\frac{\partial\left(U_{2}^{2} / U_{1}\right)}{\partial U_{1}} & \frac{\partial\left(U_{2}^{2} / U_{1}\right)}{\partial U_{2}} & \frac{\partial\left(U_{2}^{2} / U_{1}\right)}{\partial U_{3}} \\
\frac{\partial\left(U_{2} * U_{3} / U_{1}\right)}{\partial U_{1}} & \frac{\partial\left(U_{2} * U_{3} / U_{1}\right)}{\partial U_{2}} & \frac{\partial\left(U_{2} * U_{3} / U_{1}\right)}{\partial U_{3}}
\end{array}\right) \\
& A=\frac{\partial F}{\partial U}=\left(\begin{array}{ccc}
0 & 1 & 0 \\
\frac{-U_{2}^{2}}{U_{1}^{2}} & \frac{2 U_{2}}{U_{1}} & 0 \\
-\frac{U_{2} * U_{3}}{U_{1}^{2}} & \frac{U_{3}}{U_{1}} & \frac{U_{2}}{U_{1}}
\end{array}\right) \\
& A=\frac{\partial F}{\partial U}=\left(\begin{array}{ccc}
0 & 1 & 0 \\
-u^{2} & 2 u & 0 \\
-u v & v & u
\end{array}\right)
\end{aligned}
$$

Analogamente,

$$
\begin{aligned}
& B=\frac{\partial G}{\partial U}=\left(\begin{array}{ccc}
\frac{\partial U_{3}}{\partial U_{1}} & \frac{\partial U_{3}}{\partial U_{2}} & \frac{\partial U_{3}}{\partial U_{3}} \\
\frac{\partial\left(U_{2} U_{3} / U_{1}\right)}{\partial U_{1}} & \frac{\partial\left(U_{2} U_{3} / U_{1}\right)}{\partial U_{2}} & \frac{\partial\left(U_{2} U_{3} / U_{1}\right)}{\partial U_{3}} \\
\frac{\partial\left(U_{3}^{2} / U_{1}\right)}{\partial U_{1}} & \frac{\partial\left(U_{3}^{2} / U_{1}\right)}{\partial U_{2}} & \frac{\partial\left(U_{3}^{2} / U_{1}\right)}{\partial U_{3}}
\end{array}\right) \\
& B=\frac{\partial G}{\partial U}=\left(\begin{array}{ccc}
0 & 0 & 1 \\
\frac{-U_{2} U_{3}}{U_{1}^{2}} & \frac{U_{3}}{U_{1}} & \frac{U_{2}}{U_{1}} \\
-\frac{U_{3}^{2}}{U_{1}^{2}} & 0 & \frac{2 U_{3}}{U_{1}}
\end{array}\right) \\
& B=\frac{\partial G}{\partial U}=\left(\begin{array}{ccc}
0 & 0 & 1 \\
-u v & v & u \\
-v^{2} & 0 & 2 v
\end{array}\right)
\end{aligned}
$$

Obtendo os autovalores de A: $\lambda_{A 1}=\lambda_{A 2}=\lambda_{A 3}=u$ e seus respectivos autovetores $\vec{X}_{A 1}=(0,0,1), \vec{X}_{A 2}=(1, u, 0)$ e $\vec{X}_{A 3}=(0,0,0)$. E para B: $\lambda_{B 1}=\lambda_{B 2}=\lambda_{B 3}=v$ e seus respectivos autovetores $\vec{X}_{B 1}=(0,1,0), \vec{X}_{B 2}=(1,0, v)$ e $\vec{X}_{B 3}=(0,0,0)$, ou seja, $X_{A}$ e $X_{B}$ são representados pelas matrizes (A.7) e (A.8). 


$$
\begin{aligned}
& X_{A}=\left[\begin{array}{lll}
0 & 1 & 0 \\
0 & u & 0 \\
1 & 0 & 0
\end{array}\right] \\
& X_{B}=\left[\begin{array}{lll}
0 & 1 & 0 \\
1 & 0 & 0 \\
0 & v & 0
\end{array}\right]
\end{aligned}
$$

Como há a necessidade de calcular a inversa da matriz $X_{A}$ e $X_{B}$ no esquema de separação do vetor fluxo apresentado, então este método não será aplicado para o cálculo do transporte de partículas. 


\section{Quadratura Gaussiana no triângulo}

A integral (B.1) em duas dimensões num domínio triangular são expressas convenientemente em termos das coordenadas do triângulo apresentadas na Figura B.1.

$$
\iint_{\Omega_{e}} f(x, y) d x d y=A_{e} \sum_{i=1}^{n_{g}} w_{i} f\left(\alpha_{i}, \beta_{i}, \gamma_{i}\right)
$$

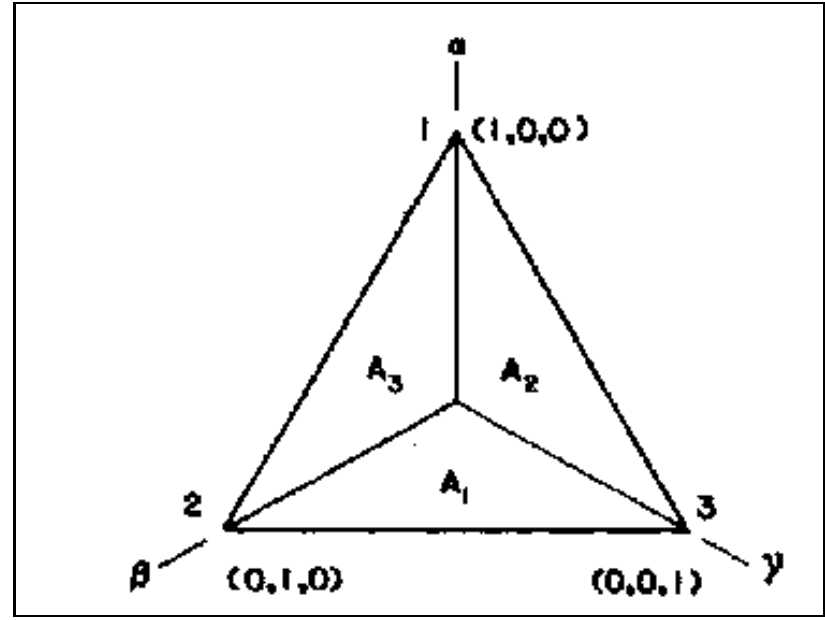

(a)

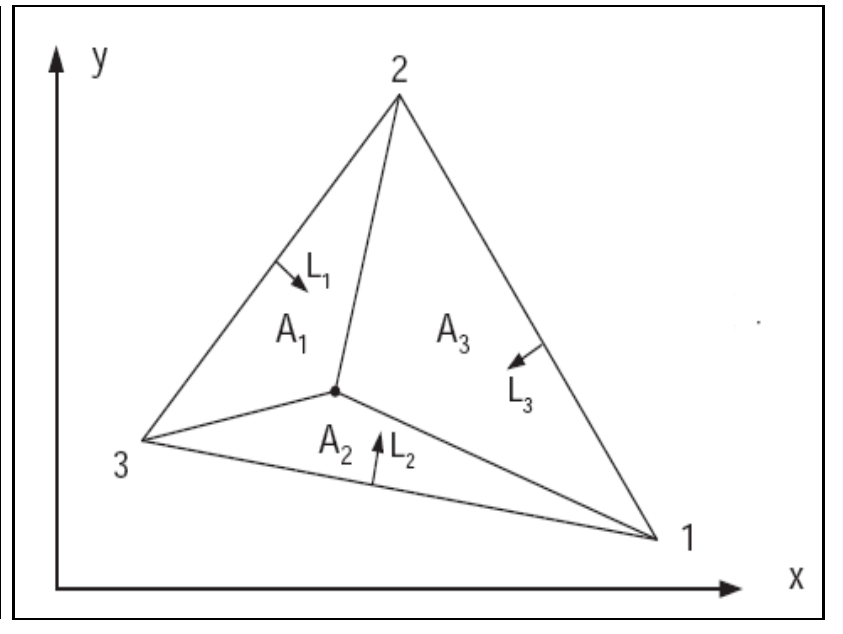

(b)

Figura B.1: (a) Triângulo natural e suas coordenadas; (b) triângulo "associado" [16].

Usualmente, a integração em um domínio triangular é realizada em coordenadas normalizadas. Para realizar a integração em um triângulo qualquer, primeiramente determinase um vértice para relacionar com a origem $(0,0)$, um segundo vértice com o ponto $(1,0)$ e um terceiro com o ponto $(0,1)$. Esta transformação é efetuada facilmente utilizando o 
vetor $\vec{r}_{a}$ do vértice 1 para o vértice 2 , o vetor $\vec{r}_{b}$ de 1 para 3 e x, y de $\vec{r}_{a}$ é dado por $\vec{r}_{a x}$ e $\vec{r}_{a y}$, respectivamente.

Então, as substituições

$$
\begin{aligned}
& x=\vec{r}_{a x}(z)+\vec{r}_{b x}(h) \\
& y=\vec{r}_{a y}(z)+\vec{r}_{b y}(h)
\end{aligned}
$$

são feitas, onde $z$ e $h$ são as coordenadas no sistema de coordenadas normalizado.

Assim, a integral em duas dimensões em um triângulo são convenientemente expressas em termos das coordenadas como

$$
\iint_{\Omega_{e}} f(x, y) d x d y=A_{e} \sum_{i=1}^{n} f\left(\zeta_{i}^{i}, \zeta_{i}^{i}, \zeta_{i}^{i}\right)
$$

onde $\left(\zeta_{i}^{i}, \zeta_{i}^{i}, \zeta_{i}^{i}\right)$ são as coordenadas triangulares do ponto de evolução i e $A_{e}$ é a área do triâgulo. Dunavant [16] apresenta fórmulas em triângulos que são exatas até ordem 20, Tabela B.1 Assim, a integração da função original e nos sistemas coordenados normalizados pode ser relacionada por

$$
\iint_{\Omega_{e}} \phi(x, y)=\sum_{k=1}^{n_{g}} A_{e} w_{k} \phi\left(x_{g}^{k} x_{A}+y_{g}^{k} x_{B}+z_{g}^{k} x_{C}, x_{g}^{k} y_{A}+y_{g}^{k} y_{B}+z_{g}^{k} y_{C}\right)
$$

onde

- $\left(x_{A}, y_{A}\right),\left(x_{B}, y_{B}\right)$ e $\left(x_{C}, y_{C}\right)$ são as coordenadas dos vértices do triângulo.

- $w_{g}^{k}$ são os pesos nos pontos de Gauss.

- $x_{g}, y_{g}, z_{g}$ são os parâmetros para determinar os pontos de Gauss a partir das coordenadas dos vértices.

QUARTERONI et al. [?] apresentam as aproximações B.2 e B.3 para o cálculo da integral em um triângulo.

$$
\begin{gathered}
\iint_{\Omega} \phi(x, y) d \Omega=\frac{A_{e}}{3} \sum_{k=1}^{3} \phi\left(x_{m}^{k}, y_{m}^{k}\right) \\
\iint_{\Omega} \phi(x, y) d \Omega=\frac{A_{e}}{60}\left[3 \sum_{k=1}^{3} \phi\left(x_{v}^{k}, y_{v}^{k}\right)+8 \sum_{k=1}^{3} \phi\left(x_{m}^{k}, y_{m}^{k}\right)+27 f\left(x_{c}, y_{c}\right)\right]
\end{gathered}
$$


onde $\left(x_{v}^{k}, y_{v}^{k}\right)$ e $\left(x_{m}^{k}, y_{m}^{k}\right)$ são, respectivamente, os vértices do triângulo e os pontos médios dos lados do mesmo e $\left(x_{c}, y_{c}\right)$ é o centróide do triângulo, dados por

$$
x_{c}=\frac{1}{3} \sum_{k=1}^{3} x_{v}^{k} \text { e } y_{c}=\frac{1}{3} \sum_{k=1}^{3} y_{v}^{k} .
$$

\begin{tabular}{|c|c|c|c|c|c|}
\hline $\mathrm{P}$ & $\mathrm{N}$ & $w_{t}$ & $L_{1}$ & $L_{2}$ & $L_{3}$ \\
\hline 1 & 1 & 1.000000000000000 & 0.333333333333333 & 0.333333333333333 & 0.333333333333333 \\
\hline 2 & 3 & 0.333333333333333 & 0.66666666666666667 & 0.1666666666666667 & 0.1666666666666667 \\
\hline \multirow[t]{2}{*}{3} & 1 & -0.562500000000000 & 0.333333333333333 & 0.333333333333333 & 0.333333333333333 \\
\hline & 3 & 0.520833333333333 & 0.600000000000000 & 0.200000000000000 & 0.200000000000000 \\
\hline \multirow[t]{2}{*}{4} & 3 & 0.223381589678011 & 0.108103018168070 & 0.445948490915965 & 0.445948490915965 \\
\hline & 3 & 0.109951743655322 & 0.816847572980459 & 0.091576213509771 & 0.091576213509771 \\
\hline \multirow[t]{3}{*}{5} & 1 & 0.225000000000000 & 0.333333333333333 & 0.333333333333333 & 0.333333333333333 \\
\hline & 3 & 0.132394152788506 & 0.059715871789770 & 0.470142064105115 & 0.470142064105115 \\
\hline & 3 & 0.125939180544827 & 0.797426985353087 & 0.101286507323456 & 0.101286507323456 \\
\hline \multirow[t]{3}{*}{6} & 3 & 0.116786275726379 & 0.501426509658179 & 0.249286745170910 & 0.249286745170910 \\
\hline & 3 & 0.050844906370207 & 0.873821971016996 & 0.063089014491502 & 0.063089014491502 \\
\hline & 6 & 0.082851075618374 & 0.053145049844817 & 0.310352451033784 & 0.636502499121399 \\
\hline \multirow[t]{4}{*}{7} & 1 & -0.149570044467682 & 0.333333333333333 & 0.333333333333333 & 0.333333333333333 \\
\hline & 3 & 0.175615257433208 & 0.479308067841920 & 0.260345966079040 & 0.260345966079040 \\
\hline & 3 & 0.053347235608838 & 0.869739794195568 & 0.065130102902216 & 0.065130102902216 \\
\hline & 6 & 0.077113760890257 & 0.048690315425316 & 0.312865496004874 & 0.638444188569810 \\
\hline \multirow[t]{4}{*}{8} & 1 & 0.144315607677787 & 0.333333333333333 & 0.333333333333333 & 0.333333333333333 \\
\hline & 3 & 0.095091634267285 & 0.081414823414554 & 0.459292588292723 & 0.459292588292723 \\
\hline & 3 & 0.103217370534718 & 0.658861384496480 & 0.170569307751760 & 0.170569307751760 \\
\hline & 3 & 0.032458497623198 & 0.898905543365938 & 0.050547228317031 & 0.050547228317031 \\
\hline
\end{tabular}




\begin{tabular}{|cccccc} 
& 6 & 0.027230314174435 & 0.008394777409958 & 0.263112829634638 & 0.728492392955404 \\
& & & & & \\
9 & 1 & 0.097135796282799 & 0.333333333333333 & 0.333333333333333 & 0.333333333333333 \\
& 3 & 0.031334700227139 & 0.020634961602525 & 0.489682519198738 & 0.489682519198738 \\
& 3 & 0.077827541004774 & 0.125820817014127 & 0.437089591492937 & 0.437089591492937 \\
& 3 & 0.079647738927210 & 0.623592928761935 & 0.188203535619033 & 0.188203535619033 \\
& 3 & 0.025577675658698 & 0.910540973211095 & 0.044729513394453 & 0.044729513394453 \\
& 6 & 0.043283539377289 & 0.036838412054736 & 0.221962989160766 & 0.741198598784498 \\
& & & & & \\
10 & 1 & 0.090817990382754 & 0.3333333333333333 & 0.333333333333333 & 0.333333333333333 \\
& 3 & 0.036725957756467 & 0.028844733232685 & 0.485577633383657 & 0.485577633383657 \\
& 3 & 0.045321059435528 & 0.781036849029926 & 0.109481575485037 & 0.109481575485037 \\
& 6 & 0.072757916845420 & 0.141707219414880 & 0.307939838764121 & 0.550352941820999 \\
6 & 0.028327242531057 & 0.025003534762686 & 0.246672560639903 & 0.728323904597411 \\
6 & 0.009421666963733 & 0.009540815400299 & 0.066803251012200 & 0.923655933587500 \\
\hline
\end{tabular}

$\mathrm{P}=$ grau do polinômio integrado exatamente, indica a ordem da regra da quadratura.

$\mathrm{N}=$ número de ciclos usados, número de permutação associada com um ponto de avaliação para o centróide do triângulo.

$w_{t}=$ peso para os pontos.

$L_{j}=$ coordenadas da área para o ponto, pontos de avaliação para integração em triângulos.

Tabela B.1: Quadratura Gaussiana no triângulo utilizando suas coordenadas e área [16]. 


\section{Recursos computacionais utilizados}

Seguem-se as máquinas que foram usadas na execução e validação do código computacional.

Máquina 1, uma Power Mac G5 (modelo M9591LL/A) com processador dual de 2.3GHz, 4GB de memória RAM, 250GB de disco rígido, aritmética de 64 bits e sistema operacional Linux (yellow dog linux - ydl). Esta máquina está localizada no Departamento de Matemática Aplicada do Instituto de Matemática e Estatística da Universidade de São Paulo, adquirida pelo prof. Dr. Alexandre Megiorin Roma através do projeto FAPESP \# 04/13781-1.

Máquina 2, uma Power Mac G5 (modelo M9592LL/A) com processador quad (duplo dual) de 2.5GHz, 16GB de memória RAM, 250GB de disco rígido, aritmética de 64 bits e sistema operacional Linux (ydl). Esta máquina está localizada no Departamento de Matemática Aplicada do Instituto de Matemática e Estatística da Universidade de São Paulo adquirida pelo prof. Dr. Alexandre Megiorin Roma através do projeto FAPESP \# 04/13781-1.

Máquina 3, Cluster Beowulf Alcatéia, constituido de um nó servidor e 32 nós clientes conectados via Ethernet, baseados em CPUs Intel Dual Xeon de 2.8GHz e 2.5GB de memória RAM, equipado com discos SCSI com 72GB para armazenamento dos usuários e aritmética de 32 bits e o cluster está localizado no Laboratório de Computação Científica Avançada (LCCA) do Centro de Computação Eletrônica (CCE) da Universidade de São Paulo. 
Máquina 4, Ultra Enterprise 3000 da Sun Microsystems para o uso do aplicativo Tecplot 7.5 - localizado nas Estações de Trabalho do do Instituto de Matemática e Estatística da Universidade de São Paulo.

Máquina 5, Genuine Intel(R) CPU T2400 1.83GHz, 1GHz de capacidade, aritimética de 32 bits e sistema operacional Linux (Ubuntu 6.06 LTS). 


\section{Referências Bibliográficas}

[1] ABGRALL, R. An essentially non-oscillatory reconstruction procedure on finiteelement type meshes: application to compressible flows. Computer Methods in Applied Mechanics and Engineering, v. 116, no. 1-4, p. 95-101, 1994.

[2] AHMAD, N.; BOYBEYI, Z. Advection-diffusion equation on unstructured adaptive grids. NOAA/EPA Golden Jubilee Symposium on Air Quality Modeling and Its Applications, 2005.

[3] AMAT, S.; ARÀndiGA, F.; COHEN, A.; DONAT, R.; GARCIA, G.; von OEHSEN, M. Data compression with ENO Schemes. GrAN - Grup de recerca d'anàlisi numèrica, GrAN report 99-03, 1999.

[4] BARTH, T. J.; JESPERSEN D. C. The Design and application of Upwind Schemes on unstructured meshes. AIAA - 27 th Aerospace Sciences Meeting, Reno, NV, Estados Unidos; 9-12 jan. 1989, 13 p., 1989.

[5] BARTH, T.J.; FREDERICKSON, P. O. Higher Order Solution of the Euler Equations on Unstructured Grids Using Quadratic Reconstruction. AIAA - 28th Aerospace Sciences Meeting, Reno, NV, Estados Unidos, 8-11 jan. 1990.

[6] BARTH, T.J. Recent Improvements in High Order K-exact Reconstruction on Unstructured Meshes. AIAA 1993.

[7] BARTH, T.J. Lecture Notes from VKI Lecture Series in CFD Course, 2003.

[8] BARTH, T.J. Lecture Notes from VKI Lecture Series in CFD Course, 1994. 
[9] BOURGault, Y.; HABASHI, W. G.; DOMPIERRE, J.; BARUZZI, G. S. A finite element method study of Eulerian droplets impingment models. International Journal for Numerical Methods in Fluids, v. 29, p. 429-449, 1999.

[10] CASTRO-DÍAZ, M. J.; HECHT, F.; MOHAMMADI, B.; PIRONNEAU, O. Anisotropic unstructured mesh adaption for flow simulations. International Journal for Numerical Methods in Fluids, v. 25, p. 475-491, 1997.

[11] CEBICI, T.; KAFYEKE, F. Aircrafting.

[12] DELERY, J.; COET, M. C. Experiments on shock-wave/boundary-layer interactions produced by two-dimensional ramps and three-dimensional obstacles, Proc. on Workshop on Hypersonic flowns for Reentry Problems, Springer-Verlag, Antibes 2226 jan. 1990.

[13] DEZITTER, F.; CHENE, G.; VIALA, S.; CALMELS, B. 3D ice accretions and aerodynamic penalties prediction on aircraft. 16th AIAA Computational Fluid Dynamics Conference, Orlando, Flórida, 2003.

[14] DOMINGOS, R. H. et al.. Analysys of a CFD Procedure for Water Collection Evaluation. 44th AIAA Aerospace Sciences Meeting and Exhibit, 9-12 January 2006, Reno, Nevada, AIAA 2006-1269.

[15] DROWLEY, W. P. Numerical advection experiments. Monthly Weather Review, v. 96, no. I, 1968.

[16] DUNAVANT, D. A. High degree efficient symmetrical Gaussian Quadrature Rules for the triangle. International Journal for Numerical Methods in Engineering, v. 21, p. 1129-1148. 1985.

[17] EBERLE, A. Efficient and Refined Transonic Flow Analysis Using a New Flux Vector Splitting Scheme.

[18] FREITAS, S. R. de. Modelagem numérica do transporte e das emissões de gases traços e aerossóis de queimadas no cerrado e floresta tropical da América do Sul. TESE DatDef 17.08.1999. IF - INSTITUTO DE FISICA. 
[19] GENT, R. W.; DART, M. P.; CANSDAlE, J.T. Aircraft icing. Defense Evaluation and Research Agency. Hampshire GU14 ULX, UK The Royal Society.

[20] GENT, R. W.; DART, M. P.; CANSDAlE, J. T. Aircraft icing. The Royal Society, v. 358 , p. $2873-2911,2000$.

[21] GENT, R. W. TRAJICE2 - A combined water droplet trajectory and ice accretion prediction program for aerofoils. Defence Evaluation And Research Agency Farnborough Hampshire GU13 0LX, User Guide V2.01. Tecnical Report TR90054, Royal Aerospace Establishment Copyright Controller HMSO, London, 1990.

[22] GOOCH, C. F. O. Multigrid aceleration of an upwind Euler solver on unstructured meshes, 1995.

[23] GOOCH, C. O.; VAN ALTENA, M. A high-order-accurate unstructured mesh Finite Volume Scheme for the advection-diffusion equations. Journal of Computational Physics, v. 181, p. 729-752, 2002.

[24] GOOCH, C. O. High-order ENO schemes for unstructured meshes based on LeastSquare Reconstruction, AIAA paper 07-05-40, 1997.

[25] GOOCH, C. O. High-order ENO schemes for unstructured meshes based on LeastSquare Reconstruction, 2003

[26] GOOCH, C. O. Quasi-ENO Schemes for unstructured meshes based on unlimited data-dependent least-square reconstruction. Journal of Computational Physics, v. 133, 1997.

[27] GOOCH, C. O.; NEJAT, A.; MICHALAK, K. On Obtaining High-Order FiniteVolume Solutions to the Euler Equations on Unstructured Meshes. 18th AIAA Computational Fluid Dynamics Conference, 2007.

[28] GREY, V. H. Correlations Among Ice Measurements, Impingment rates Icing Condition, and Drag Coefficients for Unswept NACA65A004 Airfoil

[29] HABASHI, W. G.; MORENCY, F.; BEAUGENDRE, H. A second generation CFDbased, fully 3D in-flight icing simulation system. NSERC-J.-A. Bombardier Industrial Research Chair of Multi-disciplinary CFD Computational Fluid Mechanics Laboratory mc Gill Univerity, Montreal, Canada and Newmerical Technologies International. 
[30] HAMED, A.; DAS, K.; BASU, D. Numerical simulations of ice droplet trajectories and collection efficiency on aero-engine rotating machinery. 43rd AIAA Aerospace Sciences Meeting \& Exhibit, Reno, NV, Estados Unidos; 10-13 jan. 2005, 10p , 20005.

[31] HEINRICH, A. et al. Analytical Methods. Section 2, Cap 4: Test Methods and Facilities. In: HEINRICH, A. et al. Aircraft Icing Handbook - Volume 2 of 3. Atlantic City, Federal Aviation Administration, U.S. Department of Transportation, 1991. (FAA Technical Center Report. DOT/FAA/CT-88/8-2)

[32] HIERNAUX, S.; ESSERS, J. A.; WERY, M. A Quadratic Reconstruction Scheme for the solution of Euler and Navier-Stokes Equations at very low Mach numbers. Proceedings of the Congress on Numerical Methods in Computational Mechanics, Praha, Czech Republic, 1997.

[33] HOFFMAnN, K. A.; CHIANG, S. T. Computational fluid dynamics for engineers. Engineering Education System, v.1 e 2, 1993.

[34] HORVATH, K. M. AL-KHALILAND C.; MILLER, D.R.; WRIGHT W. B. Validation of NASA Thermal Ice Protection Computer Codes - Part 3: The Validation of Antice.

[35] HU, C.; SHU C. Weighted Essentially Non-Oscillatory Schemes on triangular meshes. NASA/CR-1998-208459 ICASE Report no. 98-32, 1998.

[36] II, S.; SHIMUTA, M.; XIAO, F. A 4th-order and single-cell-based advection scheme on unstructured grids using multi-moments. Computer Physics Communications, p. $1-17,2005$.

[37] JAMESON, A. Analysis and design of numerical schemes for gas dynamics 1 artificial diffusion, upwind biasing, limiters and their effect on accuracy and multigrid convergence. RIACS Technical Report 94.15, International Journal of Computational Fluid Dynamics, v. 4, p. 171-218, 1995

[38] LALLEMAND, M. H. Schemas Decentrés Miltigrilles pour la Résolution des Equations D'Euler en Eléments Finis, Thesis, Univ. of Provence-Sain Charles, 1988.

[39] LINDQUIST, D. R. A Comparison of Numerical Schemes on Triangular and Qaudrilateral Meshes. PhD Thesis, Massachusetts Institute of Technology, 1988. 
[40] MAVRIPLIS, D. J. Revisiting the least-squares procedure for gradient reconstruction on unstructured meshes. National Institute of Aerospace, NASA/CR-2003-212683, NIA Report no. 2003-06, 2003.

[41] MOHAMMADI, B. Fluid dynamics computation with NSC2KE - an user guide. INRIA, Release 2.0 NºT-0164, 1994.

[42] MOREnCY, F.; BEAUGENDRE, H.; HABASHI, W. G. FeNSAP-ICE: A study of effects of ice shapes on droplet impingement. 41st Aerospace Sciences Meetins \& Exhibit, 2003, Reno, Nevada, AIAA 2003-1223.

[43] MORGAN, K.; PERAIRE, F. Unstructured Grid Finite Element Methods for fluid Mechanisc.

[44] NEJAT, A.; GOOCH, C. O.; MICHALAK, K. Accuracy Assessment Methodology for a High-Order Unstructured Finite Volume Solver. 18th AIAA Computational Fluid Dynamics Conference, 2007.

[45] NEJAT, A.; GOOCH, C. O. Effect of Discretization Order on Preconditioning and Convergence of a Higher-Order Unstructured Newton-Krylov Solver for Inviscid Compressible Flows. Presented at the AIAA Forty-fifth Aerospace Sciences Meeting, 2007.

[46] NEMEC, M.; ZINGG D. W. Aerodynamic Computations Using the Convective Upstream Split Pressure Scheme with Local Preconditioning.

[47] NISHIO, S.; KATO, S. Development of ice accretion and anti-icing system simulation code. 24th International Congress of the Aeronautical Sciences, 2004.

[48] OLIVEIRA, P. H. I. A.; BORTOLUS, M. V. Implementação computacional do método dos painéis para análise aerodinâmica de corpos tridimensionais com ou sem sustentação. Society of Automotive Engineers, Inc., 2002.

[49] OLIVEIRA, P. J.; ISSA, R. I. Numerical aspects of an algorithm for the Eulerian simulation of two-phase flows. International Journal for Numerical Methods in Fluids, v. 43, p. 1177-1198. John Wiley \& Sons, Ltd., 2002.

[50] PALADINO, E. E. Modelagem matemática e simulação numérica de trajetórias de derrames de petróleo no mar. Dissertação apresentada ao Departamento de Engenharia Mecânica da Universidade Federal de Santa Catarina, 2000. 
[51] PADILlA, F.; SECRETAN, Y.; LECLERCON, M. Open boundaries in the Finite Element aproximation of two-dimensional advection-diffusion flows. International Journal for Numerical Methods in Engineering, v. 40, p. 2493-2516, 1997.

[52] PERERA, M. K. H. Development and verification of the generic three dimensional finite volume solver. Master Thesis Department of Mechanical Engineering. The University of British Columbia, 2003.

[53] POTAPCZUK, M. G.; BRAGG, M. B.; KNON, O. J.; SANKAR, L. N. Simulation of Iced Wing Aerodynamics.

[54] POTAPCZUK, M. G. A Review of FASA Lewis' Development Plans for Computational Simulation of Aircraft Icing.

[55] SAITO, O. H.Um modelo matemático para tanques com agitação. Dissertação de Mestrado - IME/USP, São Paulo, 2001.

[56] SALTEL, E.; HECHT, F. EMC2 - 2D Finite Elements mesh generator. INRIA, 68p, 1995.

[57] SANTOS, L. C. C. et al.. Grid Sensitivity Effects in Collection Efficiency Computation. AIAA Aerospace Sciences Meeting and Exhibit, 2004.

[58] SANTOS, L. C. C. et al.. A Grid Refinement Strategy for Impingement Limits Computation for an Eulerian Collection Approach. AIAA Aerospace Sciences Meeting and Exhibit, 2005.

[59] SCHILICHTING, H. Boundary-Layer Theory, New York, Mc Graw Hill, Inc., ed. 7, 1979.

[60] SCHNEIDER, F. A.; MALISKA, C. R. Uma formulação em volumes finitos usando malhas não-estruturadas. $2^{\text {nd }}$ International Conference on Computational Heat and Mass Transfer, COPPE/UFRJ, 2001.

[61] SILVA, G. A. L. da. Modelagem e simulação da operação de sistema antigelo eletrotérmico de um aerofólio. Dissertação apresentada à Escola Politécnica da Universidade de São Paulo, 2002. 
[62] SILVA, G. A. L. da, SIlvARES, O. M.; ZERBINI, E. J. G. J. Airfoil Anti-Ice System Modeling and Simulation.

[63] SILVEIRA, R. A.; MALISKA, C. R. Influência das condições ambientais na formaçao de gelo na borda de ataque de um aerofólio. XVI Congresso Brasileiro de Engenharia Mecânica - COBEM 2001, Uberlândia, MG, 2001.

[64] SILVEIRA, R.; MALISKA, C. R. Numerical simulation of ice accretion on the leading edge of aerodynamic profiles. $2^{\text {nd }}$ International Conference on Computational Heat and Mass Transfer, COPPE/UFRJ, 2001.

[65] SILVEIRA, R. A.; MALISKA, C. R.; ESTIVAM, D. A.; MENDES, R. Evaluation of collection efficiency methods for icing analisys. XVI Congresso Brasileiro de Engenharia Mecânica - COBEM 2001 - COBEM 2003, São Paulo, 2003.

[66] SILVEIRA, R. A.; MALISKA, C. R. Simulação numérica da formação de gelo na borda de ataque de perfis aerodinâmicos. Dissetação apresentada à Universidade Federal de Santa Catarina, Programa de Pós-Graduação em Engenharia Mecânica, Florianópolis, 2001.

[67] SILVEIRA, R. A.; MALISKA, C. R. Previsão tridimensional da formação de gelo em perfis aerodinâmicos. Tese apresentada à Universidade Federal de Santa Catarina, Programa de Pós-Graduação em Engenharia Mecânica, Florianópolis, 2006.

[68] SONG, S.; CHEN, M. Third Order Accurate Large Particle finite Volume Method on Unstructured Trinagular Meshes. 2002 - siam J. Sci. Comput. Vol. 23, no. 5, pp. 1456-1463.

[69] SWANSON, R. C.; RADESPIEL, R.; TURKEL E. Comparison of Several Disipation Algorithms for Central Difference Schemes.

[70] VAN ALTENA, M. High-order finite volume discretizations for solving a modified advection-diffusion problem on unstructured triangular meshes. Master Thesis, Department of Mechanical Engineering, The University of Britsh Columbia, 1999.

[71] VENKATAKRISHNAN, V. Convergence to steady state solutions of the Euler equations on unstructured grids with limiters. Journal of Computational Physics, v. 118, no. 1, p. 120-130, 1995. 
[72] WHITE, F. M. Viscous Fluid Flow. New York: Mc Graw Hill, Inc., ed. 2, 1991.

[73] WIROGO, S.; SRIRAMBHATLA, S. An Eulerian method to calculate the collection efficiency on two and three dimensional bodies. 41st Aerospace Sciences Meeting and Exhibit, 2003, Reno, Nevada, AIAA 2003-1073.

[74] WRIGHT, W. B. Users Manual for the Improved NASA Lewis Ice accretion Code LEWICE 1.6

[75] Aircraft icing handbook. Safety Education and Publishing Unit, Civil Aviation Authority, New Zeand, 2000.

[76] Fluent 6.1 User's Guide. Fluent Inc., 2003.

[77] LEWICE/E: An Eulerian Based Ice Accretion Code.

[78] Trajice2 - A combined Water Droplet Trajectory and Ice Accretion Prediction Program for Aerofoils Usr Guide V2.01

[79] http://www.cira.it

[80] http://www.aerospace.bombardier.com

[81] http://www.fmi.uni-passau.de/lethrstuehle/donner/

lehvetanstaltungen/numerik_05_06/householder.c

[82] http://www.public.iastate.edu/ zjw/photos/stanford/HABASHI_ICING.pdf

[83] http://hetra-mech.ubc.ca/ANSLab/publications.html

[84] http://www.ipt.br

[85] http://www.inria.fr/inria/historique.en.html

[86] http://newmerical.com/Fensap_ice.htm

[87] http://www.onera.fr/

[88] http://people.nas.nasa.gov/ barth/

[89] http://www.tecplot.com/ 